Portland State University

PDXScholar

Fall 12-2-2013

\title{
Bismuth Nanoparticles as Medical X-ray Contrast Agents: Synthesis, Characterization and Applications
}

Anna Laura Brown

Portland State University

Follow this and additional works at: https://pdxscholar.library.pdx.edu/open_access_etds

Part of the Analytical, Diagnostic and Therapeutic Techniques and Equipment Commons Let us know how access to this document benefits you.

Recommended Citation

Brown, Anna Laura, "Bismuth Nanoparticles as Medical X-ray Contrast Agents: Synthesis, Characterization and Applications" (2013). Dissertations and Theses. Paper 1523.

https://doi.org/10.15760/etd.1522

This Dissertation is brought to you for free and open access. It has been accepted for inclusion in Dissertations and Theses by an authorized administrator of PDXScholar. Please contact us if we can make this document more accessible: pdxscholar@pdx.edu. 
Bismuth Nanoparticles as Medical X-ray Contrast Agents:

Synthesis, Characterization and Applications

by

Anna Laura Brown

A dissertation submitted in partial fulfillment of the requirements for the degree of

\author{
Doctor of Philosophy \\ in \\ Chemistry
}
Dissertation Committee:
Andrea Goforth, Chair
Carl Wamser
Dirk Iwata-Reuyl
Mark Woods
Sarah Eppley
Todd Rosenstiel
Portland State University
2013




\section{Abstract}

Bismuth based nanomaterials have recently attracted attention as heavy element X-ray contrast agents because of the high atomic number and predicted biological compatibility of bismuth. Nanoparticle X-ray contrast agents may enable a number of novel medical imaging applications, including blood pool and site-directed imaging. However these hypothetical applications are hindered by lack of suitable synthetic methods for production of imaging agents. This dissertation describes synthesis of a novel class of bismuth nanoparticles that are aqueously stabilized using poly and monosaccharides. These particles are synthesized using highly biologically compatible reagents and are oxidatively stable in water and in moderately basic buffered solutions. Bismuth nanoparticles stabilized by the polysaccharide dextran have a large hydrodynamic radius and a relatively small bismuth nanocrystal core (4\% bismuth by volume.) Glucosecapped particles have a much higher ratio of bismuth by volume $(>60 \%)$, and experimental CT scans of these particle solutions demonstrate higher X-ray contrast versus a current clinically used radiocontrast agent. Additional syntheses of hydrophobic organoamine-capped bismuth nanoparticles by reduction of an iodobismuth cluster, and development of other X-ray contrast materials, such as a radiopaque surgical sponge marker and ink, using bismuth micoparticles produced by a top-down ball milling method, are also described. 


\section{Acknowledgments}

First and foremost I would like to thank my advisor, Dr. Andrea Goforth, for advising my graduate career, teaching me how to compose written documents that are not painful to read, and how to cope with the many ups and downs (and personalities) of graduate school; mostly l'd like to thank her for taking a chance on me and on bismuth. This was supposed to be a high risk/high reward project. I sincerely hope I have conveyed in this thesis how rewarding (at least to me) this project has been.

I would also like to acknowledge my partner, Sam Noble, for listening to me talk about bismuth for the past 5 years and picking me up from the lab late at night when something was either working, or not working, particularly well. $\mathrm{He}$ has also supported and antagonized me emotionally to great effect and distraction, and fixes my computer when I (regularly) break it.

I must also acknowledge Dr. Alex Merrill and Dr. Andy Frame for their assistance with writing and for valuable laboratory advice. I would also like to thank Dr. Ben Ayres and Dr. Marilyn Rampersad Mackiewicz for their academic

and personal advice at the beginning of my graduate career; Dr. Tony Chen and Greg Baty for their help with the electron microscopes; Barb Smith at PCC for Xray assistance; Rob Jensen for help with the NMR; Will DeBenedetti for chemical and FT-IR advice; Dr. David Cormode and Dr. Pratrap Naha for CT and 
toxicology experiments; Sheng Chiu and Natasja Swartz for various instrumental and life advice.

Russ Watt and Matt Dixon and the Winter 2011 Engineering and Technology New Venture Management class helped with directing the research applications outlined in Chapter 5 of this document. Additionally, Russ and Matt have provided an excellent scientific distraction from academic research and cofounded Hawthorne Materials Corporation, that I hope will enable some of these materials to find use in the real world. *

I would also like to thank my committee, specifically Dr. Sarah Eppley and Dr. Todd Rosenstiel for advice on biological studies, Dr. Dirk Iwata-Reuyl and Dr. Carl Wamser for mechanism and carbohydrate discussions, and Dr. Mark Woods for inorganic chemistry and experimental advice.

Most importantly I would like to thank my loving, supporting and nurturing parents, Deborah and Guy Brown. My survival of graduate school, and consequently this thesis, have only been made possible because of their progressive parenting philosophy that bucks the millennia old trend of treating women as the second sex. This is a gift I am extremely grateful for and will never undervalue.

${ }^{*}$ Whatever that is. 


\section{Table of Contents}

\begin{tabular}{lc} 
& Page \\
\hline Abstract & i \\
Acknowledgments & ii \\
List of Figures & vii
\end{tabular}

Chapter 1 - Introduction and Background to Medical X-ray 1 Imaging and Bismuth as an Inorganic X-ray Contrast Material

1.1 X-ray Radiography and Molecular Contrast Agents 1

1.2 X-ray Attenuation Physics 4

1.3 Medical Contrast Agents 9

1.4 Medical Nanoparticle Contrast Agents 12

1.5 Introduction to Bismuth Nanoparticle Contrast Agents 14

1.6 Bismuth Nanoparticle Synthesis $\quad 19$

1.6.1 Top-down Synthetic Methods for Production of 20

Bismuth(0) Nanoparticles

1.6.2 Bottom up Synthetic Methods for Production of 23

Bismuth(0) Nanoparticles

1.7 Scope of this Work 50

References $\quad 51$

Chapter 2 - pH-dependent Synthesis and Stability of Aqueous, 64 Elemental Bismuth Glyconanoparticle Colloids: Potentially Biocompatible X-ray Contrast Agents

2.1 Abstract 64

2.2 Introduction 65

2.3 Experimental 69

2.4 Results and Discussion: Synthesis $\quad 74$

2.5 Results and Discussion: Discussion of Aqueous 82

Bismuth(III)oxide Chemistry

2.6 Results and Discussion: Characterization 84 
2.7 Results and Discussion: Stability and Degradation 88

2.8 Conclusion 92

References 93

Chapter 3 - Ultra-High Payload Elemental Bismuth Nanoparticle

99

X-ray Contrast Agents: Synthesis, X-ray Opacity, and Biological Compatibility

3.1 Abstract 99

$\begin{array}{ll}3.2 \text { Introduction } & 100\end{array}$

3.3 Experimental and Methods 104

3.4 Results: Synthesis of BiNPs 110

3.5 Results: Characterization of Aqueous BiNP Colloids 116

3.6 Results: X-ray Contrast and Cytotoxicity Evaluation of Glucose 121 BiNPs

$\begin{array}{ll}3.7 \text { Conclusion } & 126\end{array}$

$\begin{array}{ll}\text { References } & 127\end{array}$

Chapter 4 - Inexpensive, Aerobic Synthesis of Hydrophobic Organoamine Bismuth Nanoparticles

4.1 Abstract

4.2 Introduction to Bismuth Nanoparticle Organoamine Synthesis $\quad 136$

4.3 Synthesis and Methods 138

4.4 Results and Discussion 140

$\begin{array}{ll}4.5 \text { Conclusion } & 146\end{array}$

$\begin{array}{ll}\text { References } & 147\end{array}$

\section{Chapter 5 - Highly X-ray Opaque Polymer/Bismuth} Microparticle Composite Materials

5.1 Abstract

5.2 Introduction to X-ray Opaque Composite Materials

5.3 Methods

5.4 Results and Discussion 
5.5 Conclusions

References

Apendix A - Synthesis of Ethylene Glycol and Glucose Bismuth 204 Nanoparticles and Applications as X-ray Opaque Inks

Appendix B. - Bismuth Nanoparticles Stabilized by a Variety of 208 Reducing Sugars, and the pH Dependent Stability of Glucose Capped Particles 


\section{List of Figures}

Page

Figure 1.1

Figure 1.2

Figure 1.3

Figure 1.4

Figure 1.5

Figure 1.6

Figure 1.7

Figure 1.8

Figure 1.9

Figure 1.10

Figure 1.11

Figure 1.12

Figure 2.1

Figure 2.2

Figure 2.3

Figure 2.4
Medical X-ray images

Mass attenuation coefficients as a function of incident radiation energy for a variety of materials

Chemical structures of select iodinated X-ray contrast agents

Representative electron microscopy images and XRD patterns from top down syntheses of BiNPs

Representative TEM images of bismuth

nanomaterials produced from a bismuth(III) silylamide precursor

TEM images of BiNPs produced by the Yarema et al. method.

Representative TEM images of BiNPs produced from bismuth(III) thiolate precursors. bismuth(III) salt in DMF in the presence of PVP Representative electron microscopy images of bismuth nanomaterials produced by the polyol synthesis.

TEM images of products of polyol synthesis using a $\mathrm{NaBiO}_{3}$ precursor.

Electron microscopy images of bismuth(0) particles produced by the polyol method, from Goia et al.

TEM images of BiNPs synthesized in aqueous solvents. dependent on the reaction $\mathrm{pH}$ of bismuth nanoparticles synthesized at pH 9.97 X-ray diffraction and FT-IR absorbance of BiNPs 
Figure 2.5 Hydrodynamic diameter of BiNP populations

Figure 2.6 Stability of BiNPs in phosphate buffered solutions and exposed to light

Figure 3.1 Size distributions of inorganic core and organic shell diameter of BiNPs synthesized at $80^{\circ} \mathrm{C}$

Figure 3.2

Figure 3.3

Figure 3.4

Figure 3.5

Figure 3.6

Figure 4.1

Figure 4.2

Figure 4.3

Figure 4.4

Figure 4.5

Figure 5.1

Figure 5.2

Figure 5.3

Figure 5.4

Figure 5.5

Figure 5.6
TEM image of particle cores

Surface characterization of particles by ${ }^{1} \mathrm{H}$ NMR and FT-IR

CT attenuation evaluation of BiNPs

122

Cellular uptake and X-ray attenuation of BiNPs

123

Viability of HeLa and J774.A cells incubated with BiNPs

126

Bismuth particles synthesized by hot injection of iodobismuth precursor into neat hexadecylamine

Bismuth particles synthesized in a 1:1 hexadecane:hexadecylamine mixture

SEM images of bismuth particles synthesized in hexadecylamine and hexadecane

Representative morphologies of bismuth nanocrystals obtained when synthetic solutions at reaction temperature were not rapidly cooled

FT-IR spectrum of dried bismuth nanoparticles

146

Bimsuth particle miscibility illustrated by solvent separation

159

Illustration of microparticle synthesis, purification,

160 size selection

Polyurethane and commercially available X-ray opaque sponge markers

162

X-ray attenuation of loaded surgical sponge markers

164

Hydrophilic particles embedded in a dextran

165 polymer matrix and dried into a small disc

166 
Figure 5.7

Figure 5.8

Figure 5.9
SEM images of bismuth micron particles made by ball milling bulk bismuth in 1-pentene and pentane

Silicone and hydrophobic bismuth particle composite materials

Illustrative use of styrene inks

168

170 


\section{Chapter 1 - Introduction and Background to Medical X-ray Imaging and Bismuth as an Inorganic X-ray Contrast Material}

\subsection{X-ray Radiography and Medical X-ray Contrast Agents}

Since the discovery of X-rays (electromagnetic radiation with wavelengths between 0.01 and $10 \mathrm{~nm}$ ) in 1895 by Wilhelm Röntgen and their immediate application to medicine, X-ray radiography has become an indispensible method for noninvasive imaging of internal anatomical structures.(1) X-ray imaging is the most common internal anatomical structure imaging technique used in hospitals, and upwards of 300 million radiological exams are performed per year in the United States.(2) An X-ray radiograph is produced by a short exposure of X-ray radiation to a patient, where the partially attenuated radiation is captured on film or on a detector. Dense structures, most notably the calcium-based skeletal system, are more likely to attenuate an X-ray photon, relative to lighter, less dense structures, such as soft tissue. 

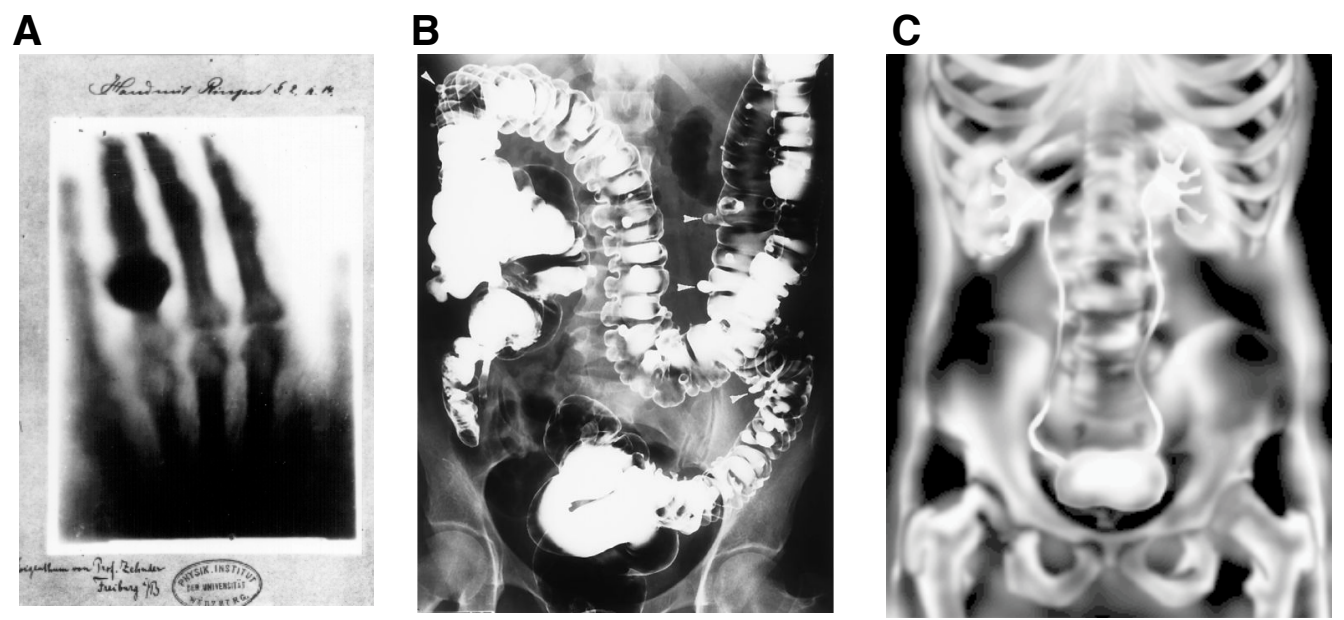

Figure 1.1 Medical X-ray images. A.) First medical X-ray taken by Wilhelm Röntgen in 1895. Note that gold ring $(Z=79$, density= $19.30 \mathrm{~g} / \mathrm{cm}^{3}$ ) is much more highly attenuating than the soft tissue of the hand and the calcium-based endoskeleton.(1) B.) Barium sulfate contrast medium used to image intestinal structures. The insoluble salt is orally or rectally administered and coats the intestines.(3) C.) lodinated contrast medium is injected into a vein, usually in the arm, during an intravenous pyelogram. The radiologist takes a series of snapshots as the medium circulates in the blood and reaches the kidneys. Functional information is captured as contrast medium is filtered from the blood and passes to the ureters.(4)

The ability of a material to attenuate $\mathrm{X}$-rays is dependent on the attenuation coefficient of the material, which is discussed in detail later. Briefly, the attenuation coefficient is primarily dependent on the atomic number $(Z)$ of the element or elements that compose the material, and on the density of the material. Different tissues types, even when composed of similar elements, can be radiographically differentiated based on the density differences between them. 
For example, water (density $=1 \mathrm{~g} / \mathrm{cm}^{3}$ ) and fatty tissue (density $=0.91 \mathrm{~g} / \mathrm{cm}^{3}$ ) can be distinguished and imaged by a skilled radiologist, enabling identification of individual organs. However, X-rays are most useful in medicine for imaging the endoskeleton, which is composed primarily of calcium $(Z=20)$, and is a primary component of compact bone (density $=1.85 \mathrm{~g} / \mathrm{cm}^{3}$ ). The first medical X-ray radiograph taken was of the hand of Anna Bertha Ludwig, the wife of Wilhelm Röntgen (Figure 1.1 A) which clearly shows a high contrast image of her finger bones and a gold ring.(1) This early X-ray radiograph demonstrates the potential to use X-rays for imaging dense and high Z element internal structures and of the X-ray attenuation potential from inorganic heavy element items, namely the gold ring on her finger.

High $Z$ reagents administered as contrast agents are commonly used in medicine to enhance the X-ray attenuation of anatomical structures (Figure 1.1 B and C) and the chemical composition and pharmacokinetics of these reagentswill be addressed in a separate section. Relatively X-ray transparent anatomic features, such as the upper and lower intestine and the kidneys, can be imaged by X-ray radiography with administration of an appropriate contrast agent. Administration of insoluble suspended contrast material, either orally or rectally, enables imaging of individual lobes of the intestine to survey for blockages, as shown in Figure 1.1 B. Soluble molecular contrast materials are administered intra-arterial or intra-catheter and are primarily filtered through the renal system and eliminated through the urine; they are thus are useful for imaging circulatory 
and renal function.(5) An example X-ray radiograph from an intravenous pyelogram is shown in Figure $1.1 \mathrm{C}$, and the functioning kidneys, ureters and bladder are all clearly imaged with the administration of soluble, molecular contrast media.(4)

Modern X-ray based technologies, such as Computerized Tomography (CT) have allowed for higher resolution and 3-D images to be rendered, relative to 2-D X-ray radiographs. CT scanning technology was introduced in the 1970 s. This technique scans the patient using a moving X-ray source coupled with moving detectors. Attenuated radiation information collected over a range of positions is sent to a computer that re-constructs a multilayered image of the internal structures of the patient. Allan M. Cormack and Godfrey N. Hounsfeld were awarded the Nobel Price in Physiology or Medicine in 1979 for the "development of computer assisted tomography"(6) and today roughly 80 million CT scans are performed each year in the United States.(7)

\subsection{X-ray Attenuation Principles}

The X-ray attenuation of a material is dependent on its X-ray attenuation coefficient, $\boldsymbol{\mu}$, that is defined as

$$
\mu=\rho Z^{4} / A E^{3}
$$

where $\rho$ is the density of the material, $Z$ is atomic number, $A$ is atomic mass of its elemental constituents, and $\mathrm{E}$ is incident X-ray energy. The X-ray attenuation coefficient is calculated from the sum of radiation scattering through a material 
from Rayleigh scattering, Compton scattering and the photoelectron effect. Because the $\boldsymbol{\mu}$ is an extensive property, the X-ray attenuation from atoms or materials is additive over a distance, and if the atomic composition is known for a material, the attenuation of the material can be derived by addition of the attenuations from the individual elemental components. $\boldsymbol{\mu}$ is used to calculate the fraction of attenuated radiation through a given material according to the following equation:

$$
I / I_{0}=e^{[-(\mu / \rho) x]}
$$

Where $\mathbf{I}$ is the intensity of transmitted radiation, $\mathbf{I}_{\mathbf{0}}$ is the incident radiation intensity, $\boldsymbol{\mu}$ is the $\mathrm{X}$-ray attenuation, $\boldsymbol{\rho}$ is the density, and $\boldsymbol{x}$ is the thickness of the material $(\mathrm{cm}) .(8)$ Thus $1-(\mathrm{l} / \mathrm{lo})$ is the fraction of radiation that is that is attenuated by the material as a function of the thickness. The mass attenuation coefficient $(\mu / \rho)$ is a useful term for comparing the X-ray attenuations of given elements or materials as a function of weight. Additionally, comparing the X-ray attenuation of elements or materials by volume can be achieved by calculating $\mathrm{x}$, which is the linear distance the X-ray radiation will pass through the material, from the total volume of the material.

Notably from the X-ray attenuation equation, the fraction of attenuated radiation is exponentially dependent on the atomic number of the atoms in the material, since $\boldsymbol{\mu}$ is proportional to $Z^{4}$. Thus the calculated $\boldsymbol{\mu}$ value is inherently dominated by the $\mathbf{Z}$ term. $\mathbf{Z}$ values for non-radioactive elements range from 1-83, 
and the higher $\mathbf{Z}$ values, such as for gold, lead and bismuth, will have a more dramatic exponential effect on the X-ray attenuation.

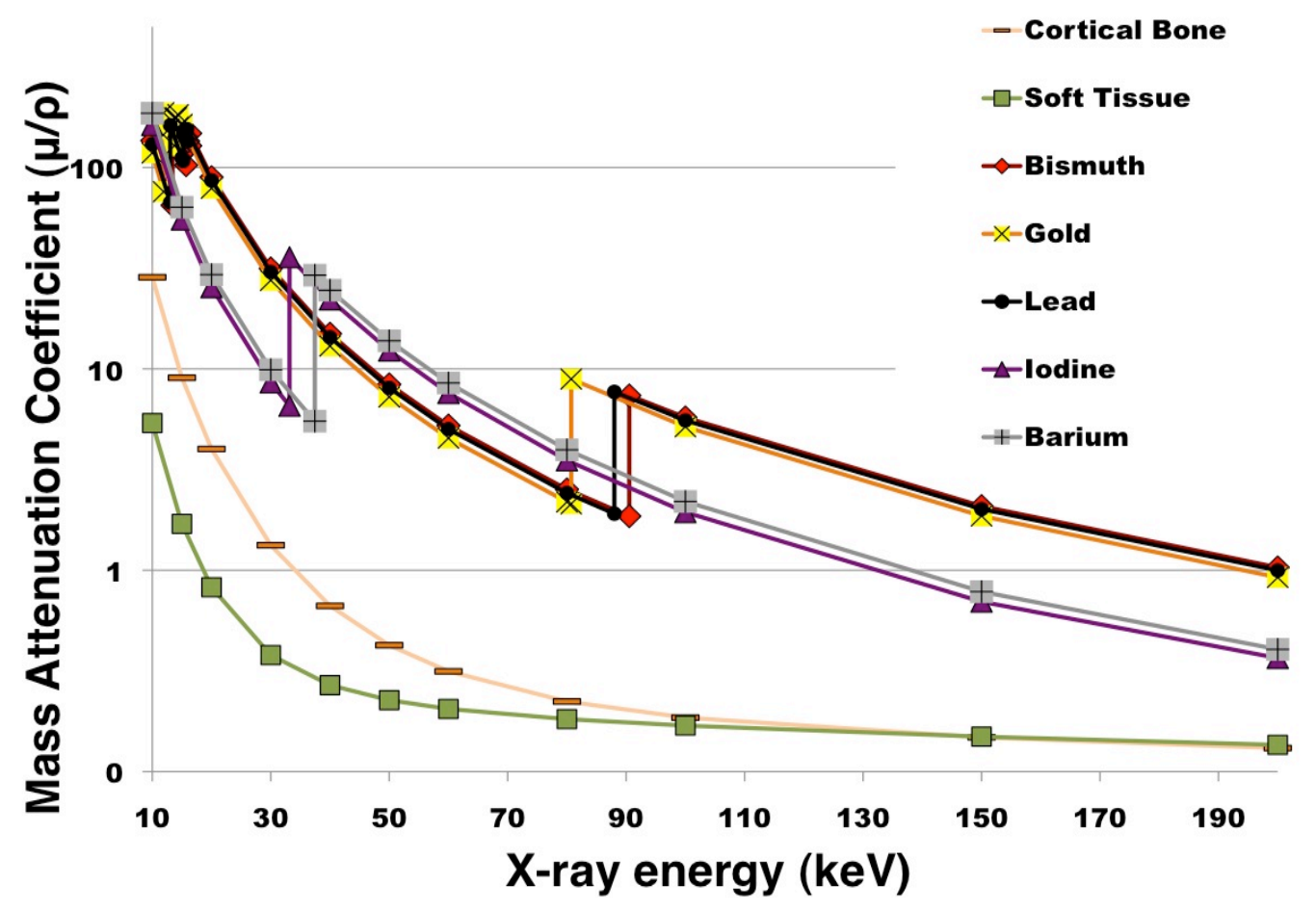

Figure 1.2. Mass attenuation coefficients as a function of incident radiation energy for a variety of materials. Data graphed from reference (8). Notably gold, lead, and bismuth have similar mass attenuation coefficients.

A graph of the mass attenuation coefficients $(\boldsymbol{\mu} / \rho)$ for elements and materials relevant to this thesis, as a function of medically diagnostic incident $\mathrm{X}$ ray energy (in keV), is shown in Figure 1.2. Bismuth, lead and gold have similar mass attenuation coefficients, primarily because their $Z$ values are $\operatorname{similar}(Z=83$, 82 and 79 , respectively), but have inversely proportional densities ( $\rho$ ) relative to their $Z$ values (density $=9.75,11.35$, and 19.32 respectively). Barium and iodine 
( $Z=56$ and 53 , respectively) have similar atomic numbers and thus similar mass attenuation coefficients relative to each other.

The K-edge energies, which are a function of the photon binding energies of the K-shell electrons in these elements, are evident in the mass attenuation coefficient vs. X-ray energy graphs as dramatic increases in X-ray attenuation at radiation energies just above this binding energy. The K-edge energies for the elements described in this section are spread out over the medically diagnostic imaging X-ray imaging range, which are $37.4 \mathrm{keV}$ for barium, $33.2 \mathrm{keV}$ for iodine, $80.7 \mathrm{keV}$ for gold, $88.0 \mathrm{keV}$ for lead, and $90.5 \mathrm{keV}$ for bismuth.(8) At X-ray energies above an element's K-edge energy, the increased X-ray attenuation coefficient leads to a markedly increased X-ray attenuation. Consequently barium and iodine, despite their lower $Z$ values relative to gold, lead and bismuth, have higher mass attenuation coefficients between roughly 40-80 keV. However, because the mass attenuation coefficient is correlating the X-ray attenuation as a function of mass, the substantially higher density of gold, bismuth and lead, particularly in their elemental form relative molecular or ionized iodine and barium, does not make iodine and barium superior contrast materials in this energy range by volume.

The increase in X-ray attenuation at energies above the K-edge for various materials is commonly taken into account by the radiologist to optimize contrast, particularly when contrast materials are administered and used to image specific anatomical structures. The subtraction of X-ray attenuation information 
acquired for a particular element immediately above and below it's K-edge energy is an emerging imaging technique to computationally produce "color" $\mathrm{X}$ ray radiographs, or reconstructed images by detecting specific elements based on this dramatic change in X-ray attenuation just above a characteristic voltage. Color CT scanners are expected to be on the market in the next decade, and will be medically useful primarily in conjunction with contrast materials that have Kedges which are distinct from surrounding materials.(9)

The K-edges for gold, lead and bismuth occur at X-ray energies commonly used for chest and pelvis radiographs. CT scans are typically performed substantially above these $\mathrm{K}$-edge energies, commonly at $150 \mathrm{keV}$, but also at $120 \mathrm{keV} .(7)$ Subsequently, medical X-ray imaging techniques using gold, lead or bismuth contrast materials will prove useful X-ray contrast materials when the $\mathrm{X}$ ray energy used is above these K-edges, but will show less dramatic X-ray attenuation properties below the K-edges. The location of the K-edge energies for the specific contrast materials is useful information for radiologists who need to adjust imaging parameters to optimize contrast in radiographs.

The physical properties of the elements described in these sections will be important for development of novel X-ray contrast materials. Particularly the location of the K-edge energies relative to diagnostic $\mathrm{X}$-ray energies used in particular medical applications is important for material optimization. Gold, bismuth, and lead have similarly high $Z$ values, but different densities. These density differences will an affect on the X-ray attenuation by volume, but not by 
weight of the contrast material. Thus the specific application or medical technique will need to be considered when selecting the elemental composition of an X-ray contrast material.

\subsection{Medical X-ray Contrast Agents}

X-ray contrast agents (XCA) are atoms, molecules or compounds, that are introduced internally to a patient for the purposes of imaging structures or tissue types that are largely X-ray transparent, typically by increasing the X-ray attenuation. Currently used clinical XCA include barium sulfate $\left(\mathrm{BaSO}_{4}\right)$ for gastrointestinal (Gl) imaging and small organic molecules with iodine substituents for imaging circulatory and renal systems. $\mathrm{BaSO}_{4}$ is a water insoluble salt, which is administered orally or rectally to a patient, and the white chalky suspension coats the patient's Gl tract rendering it X-ray opaque (Figure 1.1 B).(3) While ionic barium is fairly toxic, the insolubility of barium sulfate limits biological availability and thus this contrast material generally passes through a patient's GI tract with no adverse consequences.(10)

lodine based XCA are water soluble molecules that can be injected into a patient's circulatory system to provide increased X-ray opacity to either the renal system (Figure $1.1 \mathrm{C}$ ) or to other circulatory structures, including direct intraarterial injection for cardiac imaging. All iodine based contrast agents are variations of a 1,3,5-triiodo benzene ring with additional side chains to increase

water solubility (Figure 1.3). Medically administered solutions contain up to 
$350 \mathrm{mg} / \mathrm{mL}$ iodine in the injectable contrast media. $(5,11)$ Side chains can be ionic or non-ionic, and the water soluble units are either monomers or dimers of the triiodo benzene base. Chemical structures of some clinically used contrast agents are illustrated in Figure 1.3. Monomeric iopramidol and diatrazoic acid contain three iodine atoms per molecule, and dimeric molecules iodixanol and ioxogalallic acid each contain six iodine atoms. Trimeric molecules have also been synthesized and studied, however solutions of these compounds have a generally higher viscosity than solutions of monomeric and dimeric contrast molecules.(12)

lodine-based contrast agents are generally divided into groups of high osmolality and low osmolality, depending on side chain variants. Because of the high injection volumes (up to $35 \mathrm{~mL}$ ) required to generate adequate contrast, the osmolality of the contrast material is an important consideration for the circulatory stability of the patient. Patients commonly experience moderate and mild negative medical reactions from contrast media administration, e.g. edema, and less commonly patients will experience serious and fatal adverse drug effects, such as anaphylaxis and cardiac arrest.(13, 14) Between 1978 and 1994 the Food and Drug Administration reported 920 deaths from an estimated 170 million contrast-medium enhanced radiological studies.(15) The osmolality of the injected solutions appear to influence differences observed in renal toxicities between the various contrast agents, but other factors, such as viscosity, may also affect contrast agent induced nephropathy. $(16,17)$ 


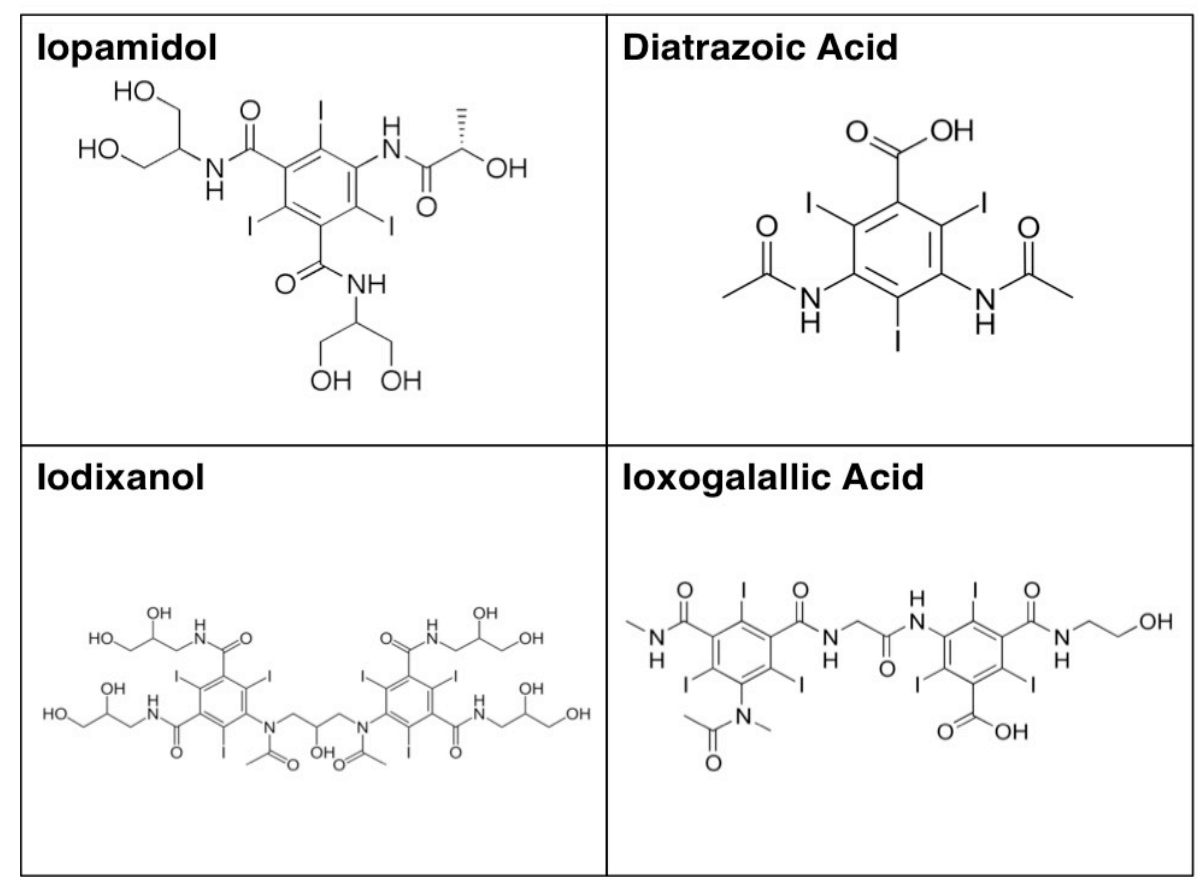

Figure 1.3. Chemical structures of select molecular iodinated XCAs. All iodoinebased XCAs are 1,3,5-triiodo benzene rings with side chain modifications to improve water solubility. lodine-based XCAs are monomeric (iopamidol and diatrazic acid) or dimeric (iodixanol and ioxogalallic acid) and are further classified as ionic (diatrazic acid and ioxogalallic acid) or non ionic (iopamidol and iodixanol).

Soluble molecular iodine-based XCAs are quickly filtered by the kidneys, and taking advantage of this, radiologist can use this information for the assessment of renal function. However, rapid renal filtration results in a short imaging window and the large volumes of administered contrast media required for imaging coupled with the clearance burden on the kidneys, these molecular XCAs cannot be administered to renally compromised patients. Recent research in iodine-based XCAs has included aggregation of clinically used XCA molecules 
into organic polymer nanoparticles in efforts to increase contrast agent circulation times (by avoidance of kidney filtration) and decrease negative responses to the administered contrast materials by decreasing osmolality of the contrast solution, and the filtration burden on the renal system. $(18,19)$

\subsection{Medical Nanoparticle X-ray Contrast Agents}

Medical XCA are currently a popular research area. The theoretical advantages of nanoparticle XCA relative to molecular or ionic salt based XCAs, include: a larger signal per unit of contrast material (a factor when osmotic pressure is a concern), longer circulation times due to size and molecular weight, opportunity to tailor surfactants to minimize adverse biological response (e.g. functional group display and surface charge) and the potential for site directed imaging by the addition of biological targeting groups to the particle surface for site or tissue specific imaging.(20) A nanoscale XCA that is large enough to avoid filtration by the kidneys would increase circulation time relative to small molecule iodine-based molecular XCAs, which have a short imaging window. This increased imaging window would perhaps allow other systems or tissues to be imaged, decrease the necessary and sometime repeat injection volumes, and enable administration of XCA to patients with compromised renal function.(21, 22) One study using cadmium selenide quantum dots, found that mouse kidneys were unable to filter particles larger than $6 \mathrm{~nm}$ from circulation, indicating that any imaging agent larger than this size should escape normal kidney filtration.(23) In 
vivo experiments using gold and bismuth based nanoparticle XCAs indicate that the lymph system will scavenge particles too large to be filtered through the kidneys on a time scale of hours or days.(24) Possible alternate clearance routes for nanoparticle vs. molecular XCAs are a potential primary advantage of these novel materials, but also a potential toxicity concern, particularly when the XCA is composed of heavy elements not typically available biologically. $(25,26)$

A number of materials have been explored as nanoparticle XCA, including $\mathrm{Bi}_{2} \mathrm{~S}_{3}$ nanoparticles wrapped in organic polymers, $(27,28)$ organic bismuth salts aggregated with polymers into nanoparticles $(29,30)$ gold nanoparticles (AuNPs)(31, 31, 32), PEGylated ytterbium oxide particles doped with erbium (33) and particles with a core composed of tantalum oxide. $(34,35)$ Studies exploring nanoparticle XCAs in vivo have primarily focused on AuNPs, and these reports have demonstrated that inorganic nanoparticle XCA have a long circulation time and that the particles ultimately localize in the spleen and liver.(36) The chemical stability of AuNPs has also enabled a number of proof-of-principal studies for site directed imaging. $(24,37)$ However the chemical stability of AuNPs has become their own barrier to application, as particles appear to bioaccumulate, which, coupled with the extremely high cost of gold, limits further development of AuNPs as XCAs. $(38-40)$ 


\subsection{Introduction to Bismuth Nanoparticles as Medical X-ray Contrast Agents}

This thesis is focused on the development of novel nanoscale BiNP XCAs with a high density and large concentration of X-ray opaque atoms, using biologically compatible reagents and solvents. These high payload nanoscale XCAs are developed to be used as biological imaging agents, and thus a synthetic focus on biological compatibility is a primary concern. Because of recent toxicity reports for AuNPs XCAs arising from biological accumulation associated with the chemical stability for AuNPs, the development of BiNP XCAs materials may be advantageous because of a lowered chemical surface stability for BiNPs and known biological compatibility for bismuth(III) species.

The ideal BiNP XCA will be between 10 and $200 \mathrm{~nm}$ to allow for free passage through the circulatory system, but avoid filtration by the kidneys.(23) The choice of elemental bismuth as an inorganic nanoparticle core for the functional atoms in an XCA, comes from the unique chemical and physical properties of this element. Bismuth-209 is the only natural isotope and is also the highest atomic number, negligibly-radioactive isotope with an alpha emission half life of $2 \times 10^{19}$ years.(41) Furthermore bismuth is moderately abundant and inexpensive, which are relatively rare characteristics of elements of the $4^{\text {th }}$ and $5^{\text {th }}$ periods.(42) Uniquely among the high $Z$ elements, bismuth has a long history of known biological tolerance and medicinal use. These properties make bismuth 
the heaviest $Z$ element that can be used as an XCA, that, coupled with potential for biological compatibility, makes bismuth ideally suited to this application.

Bismuth's low toxicity, coupled with its known anti-microbial properties, have made it commonly used in medicine for millennia.(43) For example PeptoBismol is a suspension of bismuth(III) subsalicylate that has been marketed for over a century as an anti-emetic and general treatment for gastrointestinal problems. Originally, Pepto-Bismol was developed in New York as a treatment for children suffering from undefined intestinal illness characterized by diarrhea and upset stomach, and contained red food coloring to appeal to children. Today upwards of $60 \%$ of US households have a form of Pepto-Bismol, and it is one of the most commonly used over the counter medications world wide.(44) The antimicrobial properties of bismuth(III) compounds have been demonstrated, but the biological mechanism responsible for this property is still poorly understood. Laboratory and medical research have established the role of bismuth(III) in suppression of Helicorbacter pylori growth and infection rates, which has continued to make bismuth(III) based medicines extremely popular. Some evidence indicates that bismuth(III) ions prevent protein synthesis in pathogenic microbes, and it has also been suggested that bismuth(III) ions, precipitated as bismuth oxide, form a barrier in the Gl tract, which physically bars microbes from adhesion.(45) Other studies suggest that the cationic nature of bismuth(III) ions is responsible for disruption of normal cell-wall forming function. New organic bismuth(III) drugs are currently under exploration and development. These efforts 
have shown that the bioavailability and the biological localization of bismuth(III) ions is dependent on the organic ligand component, specifically on its hydrophilicity or hydrophobicity (modes of action for bismuth(III) compounds and novel bismuth(III) drugs are reviewed in references 46-52). Generally, the literature does not agree on the specific anti-microbial mechanism, or mechanisms, of bismuth(III) ions in vivo, but all agree it is one of the most effective treatments for $H$. pylori infection and eradication.

The postulated fate of ingested bismuth(III) subsalicylate is intestinal decomposition into salicylic acid and bismuth oxide, bismuth carboxylate and bismuth oxychloride.(46) These insoluble bismuth(III) salts primarily pass through the GI tract without absorption, however some free bismuth(III) is found in the circulatory system. High plasma levels of bismuth(III) have been associated with encephalopathy and a "safe" bismuth(III) blood concentration of $<100 \mu \mathrm{g} / \mathrm{mL}$ has been established as a clinical patient toxicity threshold. Furthermore, general medical opinion has established that bismuth(III) blood levels below $50 \mu \mathrm{g} / \mathrm{mL}$ are unlikely to cause any adverse health effects.(48) One study reported that an apparently healthy volunteer, who consumed gram scale quantities of bismuth subsalicylate over 30 days, reached a peak $13.6 \mathrm{ng} / \mathrm{mL}$ serum concentration with no adverse medical effects.(53) Another study followed patients who consumed tripotassium dicitrato bismuthate tablets, and found they reached a peak bismuth plasma concentration of $50-100 \mathrm{ng} / \mathrm{mL}$.(54) Encephalopathy from prolonged bismuth(III) ingestion has been reported in the literature, but notably all reports 
indicate that the symptoms are reversible and that no lasting effects have been reported when bismuth(III) levels return to normal $(2-11 \mathrm{ng} / \mathrm{L}) .(48,55,56)$

Attempts to evaluate bismuth(0) toxicity in rats determined an $L_{50}$ of $>2,000 \mathrm{mg} / \mathrm{kg}$ for ingested $10 \mu \mathrm{m}$ elemental bismuth powders, and no negative reactions were observed when amounts of $1,000 \mathrm{mg} / \mathrm{kg}$ were ingested.(57) While the literature is varied and inconclusive about the intravenous and ingested toxicity concentrations, it is noted that the toxicity is expected to be inherently linked to the solubility of bismuth(III) ions. It is thus likely that bismuth(0) particle toxicity will likely be particle size and environment dependent, and will ultimately be dependent on the liberation of bismuth(III) ions by in vivo oxidation.

To use elemental BiNPs as long circulating XCAs, ideally the inorganic elemental BiNP will be administered to maximize X-ray opaque payload and allowed to freely circulate with a long half-life, before clearance or decomposition. Toxicity of BiNP XCAs will most likely be a function of the soluble bismuth(III) species evolved from the particles, free radicals evolved from the conversion of elemental bismuth to bismuth(III) species, and any organic component of the particles which are released as particles decompose. Other toxicity concerns for BiNP XCA will be from particle aggregation in circulation, immune responses to the administered materials, or surface interactions with specific tissue types or structures. However, these responses will be almost impossible to predict and will thus require empirical evidence and experimentation. The characterization of all components, including starting materials and the materials evolved from the 
decomposition of particles, will be the most likely in vitro evidence to enable predictions of BiNP biocompatibility. Thus using known biologically compatible starting reagents, and avoiding reagents with known toxicities that may subsequently be released during BiNP decomposition, will produce the most potentially biocompatible XCA.

Syntheses of extremely high surface area nanoparticles are generally carried out in coordinating solvents, which makes them very difficult to purify from the reaction solvent. Bismuth starting materials are also challenging, as bismuth is insoluble in most solvents, and only in water at dilute concentrations and under acidic conditions. Counterions for bismuth(III) compounds are also need to be considered during synthesis because of their role in either particle formation, particle stabilization, or additional and unintended reactions. For example $\mathrm{BiCl}_{3}$ and bismuth(III) nitrate have identical oxidation states and are both known to disassociate, however the chloride ion and nitrate ion may behave very differently in a reaction and effect the final BiNP product. Because the focus of this thesis is on the development of biocompatible elemental BiNPs, all reagents, starting materials, and the chemical composition of the resultant particles are considered. Particular focus is given to capping agents of BiNPs, and the oxidative stabilization of these particles in aqueous solutions. 


\subsection{Literature Bismuth(0) Nanoparticle Synthetic Preparations}

The bismuth(0), bismuth(III) and bismuth(V) precursors, organic ligands, and polymers that published literature preparations have reported for the synthesis of BiNPs vary widely. Additionally, reported preparations use a variety of solvents, reducing agents (for bottom up synthesis), and surfactants or capping agents. This section will discuss previously published methods for the synthesis of nanoscale bismuth(0) materials and some of the implications that physical and chemical synthetic parameters will have on the final morphology of the nanomaterials. One particular detail that will not be addressed in the following analysis is the effect that the chain length of the common surfactant poly(vinylpyrroldone) (PVP) will have on nanoparticle morphology. This particular synthetic detail may have implications for the selective growth of bismuth(0) nanowires instead of bismuth(0) nanoparticles, in addition to other physical and chemical factors. Discussions herein will focus on the identity of the chemical reagents used to produce bismuth(0) nanoparticles, and how these reagents, in addition to some physical parameters, will direct morphology of the final materials.

As nanoparticle syntheses can be generally organized into two types of methods (top down and bottom up), nanoparticles produced by these two methods will be discussed separately. Representative TEM images from prior literature preparations will be presented, but it should be noted that images 
selected for publication may not be representative of the entire population of particles produced by these methods.

\subsubsection{Top-down Synthetic Methods for Production of Bismuth(0)} Nanoparticles

A top down nanomaterial synthetic technique is defined as the physical reduction in size of a bulk material to a finer grain-sized material having at least one dimension on the nanoscale. For the synthesis of BiNPs, this entails converting bulk elemental bismuth(0) to smaller bismuth(0) particles, which offers the potential to produce large quantities of inexpensive particles as no additional reagents are required for an oxidation state change (see Chapter 5 for a detailed schematic of a top-down synthesis protocol). Top-down synthetic methods for bismuth nanomaterials are achieved either by physical fracture of the bulk material (grinding) or melting the bulk material and stirring in a solution with a surface stabilizing molecule to produce small liquid droplets that solidify on cooling (solution phase synthesis.) Formation of nanoparticles by a top-down solution phase synthesis in high boiling point organic solvents is unique to low melting point elements. Elemental bismuth(0) has a relatively low melting temperature $\left(271^{\circ} \mathrm{C}\right)$, and in general, nanoparticles are predicted to have a depressed melting temperature relative to the bulk material. 

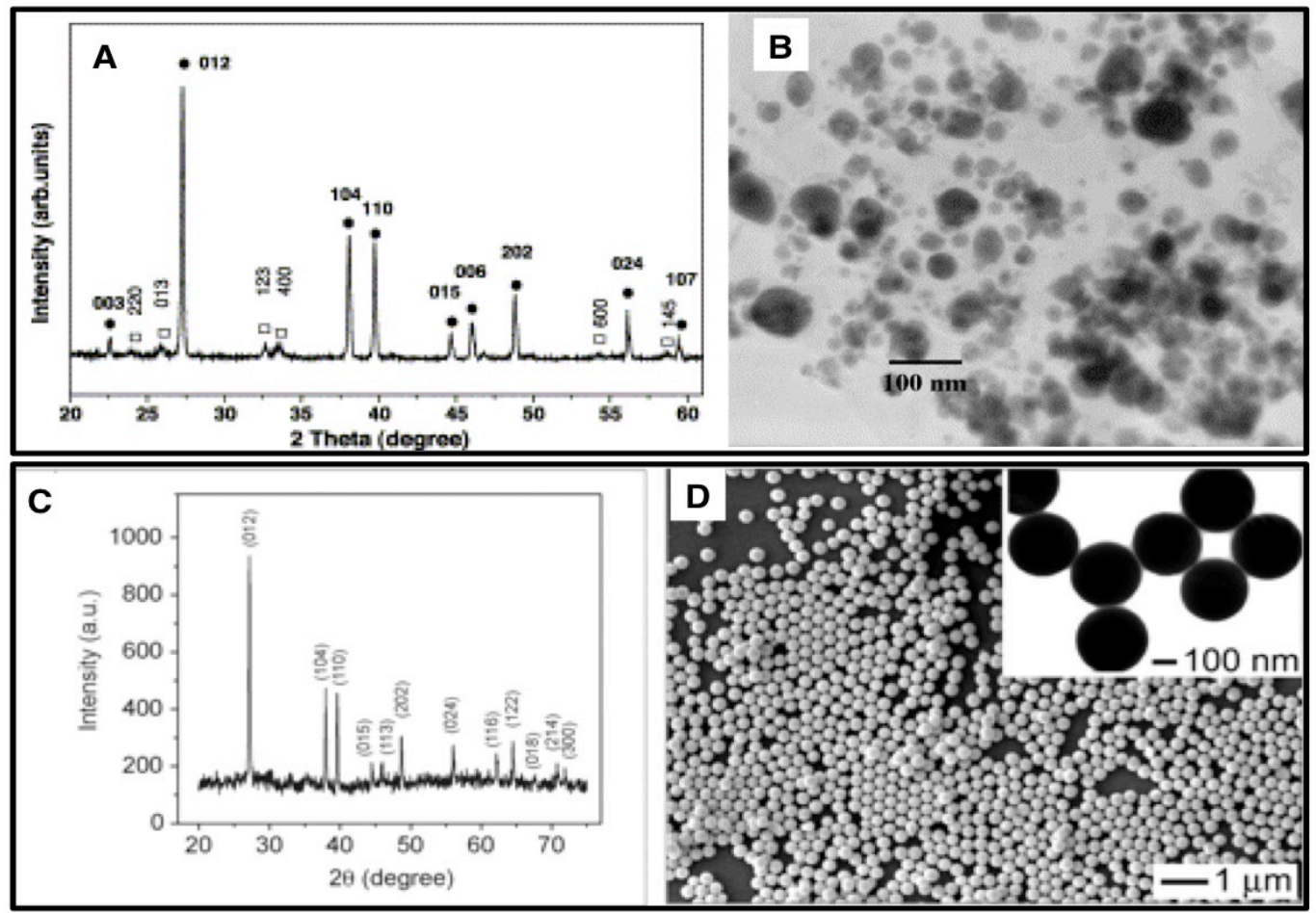

Figure 1.4. Representative electron microscopy images and XRD patterns from top down syntheses of BiNPs. Zhao et al. report a synthetic method whereby elemental bismuth is melted in paraffin to produce nanoparticles. The XRD pattern (A) contains peaks assigned to elemental bismuth (*) and bismuth(III) oxide ( $\square$ ). A TEM image of particles shows a highly polydisperse population (B).(58) Wang and Xia report the synthesis of BiNPs by melting bismuth(0) powders in diethylene glycol and PVP. The XRD pattern (C) matches rhombohedral bismuth and particles are morphologically uniform and monodispersed when imaged by SEM (D) and TEM (D inset).(59)

Two published top-down bismuth particle syntheses will be discussed here. The first preparation was carried out by heating bismuth(0) granules in paraffin in a sealed contained for 10 hours with stirring at $280{ }^{\circ} \mathrm{C}$ to melt the bulk 
bismuth in a contained environment.(58) The soluble bismuth particulate material was washed with chloroform to remove excess paraffin, and the BiNPs were collected. The X-ray diffraction pattern of the washed particles contains peaks from both rhombohedral bismuth and bismuth oxide $\left(\mathrm{Bi}_{2} \mathrm{O}_{3}\right)$ (Figure $\left.1.4 \mathrm{~A}\right)$ and the authors note that this suggests that that an oxide layer is formed on the surface of the particles. Shown in Figure $1.4 \mathrm{~B}$ are particles obtained by this method. Because paraffin is a hydrocarbon, it was assumed that the oxide formed on the particle surface is from air, or perhaps a solvent contamination. The adsorption of environmental oxygen is well noted for molten bismuth. The particles produced by this first method are heterogenous and this preparation appears to provide poor morphology control of the resultant material, which is unsurprising given the lack of surfactant included in the synthesis.

A second published method for top-down particle synthesis was carried out by melting bismuth powders (100 mesh) in diethylene glycol in the presence of PVP at the boiling point $\left(241^{\circ} \mathrm{C}\right)$ of the solvent.(59) Particles produced by this method were found to have $10 \mathrm{~nm}$ PVP coating by comparing the TEM core size to the SEM particle size, and XRD analysis (Figure $1.4 \mathrm{C}$ ) indicates an entirely elemental bismuth core, with no evidence of an oxide layer on the particle surface. This would suggest that PVP was able to effectively surface passivate BiNPs and prevent the formation of a bismuth(III) oxide layer on the particle surface. These particles are morphologically well controlled and appear highly monodispersed (Figure 1.4 D). The authors show that particles with a diameter 
between 100 and $450 \mathrm{~nm}$ can be synthesized by changing the stirring rate during particle synthesis, and they suggest that during particle formation bismuth(0) in a liquid state in solution, and thus the stirring rate will affect the final particle size. The particles were rapidly cooled from the synthetic temperature to room temperature by the addition of cold ethanol. This rapid temperature quench is a technique often used in nanoparticle synthesis to prevent particle ripening, fusing or crystal aggregation during the temperature decrease post synthesis.

The use of top-down methods for nanoparticle syntheses is appealing because of the simplicity of the chemistry and the lack of redox chemistry required. For applications of highly X-ray attenuating material, where large quantities of small particles are desired, but the morphology and polydispersity of the particle population is not critical, a top-down method should be well suited. Discussed in Chapter 4 of this dissertation is a method for producing large quantities of micron scale bismuth particles by a top-down grinding method and their incorporation into polymers to produce $\mathrm{X}$-ray opaque composite materials.

\subsubsection{Bottom-up Synthetic Methods for Production of Bismuth(0)} Nanoparticles

Syntheses of BiNPs by bottom up methods generally entail reduction of a bismuth cation (3+ or $5+)$ by a reducing agent, the aggregation of bismuth( 0$)$ atoms into nanoscale clusters, and the growth and stabilization of these clusters 
in solution. Scheme 1.1 gives a general illustration for the synthesis of bismuth(0) nanoparticles from bismuth(III) ions. Commonly used bismuth(III) sources include $\mathrm{BiX}_{3}$ where $\mathrm{X}=\mathrm{F}, \mathrm{Cl}, \mathrm{Br}$ or $\mathrm{I}, \mathrm{Bi}_{2} \mathrm{O}_{3}, \mathrm{Bi}_{2} \mathrm{~S}_{3}, \mathrm{Bi}\left(\mathrm{NO}_{3}\right)_{3} \cdot 5 \mathrm{H}_{2} \mathrm{O}, \mathrm{Bi} 5 \mathrm{O}(\mathrm{OH})_{9}\left(\mathrm{NO}_{3}\right)_{4}$ $\mathrm{Bi}(\mathrm{SR})_{3}$ and $\mathrm{Bi}(\mathrm{OR})_{3}$, where $\mathrm{OR}$ is an oxygen containing organic ligand with a negative charge, such as an acetate or a deprotonated alcohol. The only bismuth $(\mathrm{V})$ precursor discussed in this dissertation is $\mathrm{NaBiO}_{3}$, and the use of this precursor is confined to two studies. Chemical species that provide electrons to produce bismuth(0) from bismuth(III) and bismuth(V) vary, and include traditional reducing agents, such as organoboranes or hydrazine hydrate. Alternately, a general reducing solution environment, such as one generated by a polyol solvent at elevated temperature, (i.e. a polyol synthesis) can be used to produce bismuth(0). Organic surfactant species used to cap and stabilize nanomaterials also vary widely, but are generally either small molecular species (such as a primary organoamines, or carboxylic acids) or larger polymeric species, specifically PVP. By far the most commonly used surfactant is PVP, most likely because the amide functional group has a resonance structure that contains a partially negatively charged oxygen atom capable of electron donation for stabilization of a nanoparticle surface. Other surfactants reported are primary amines, $\left[\mathrm{N}\left(\mathrm{SiMe}_{3}\right)_{2}\right]^{-}$and primary carboxylic acids, such as oleic acid. These surfactants all contain a lone pair of electrons and can act as ligands for coordinating to the particle surface and providing stabilization. The following sub- 
sections discuss published bottom up preparations of BiNPs, grouped generally by class or by reagents used.

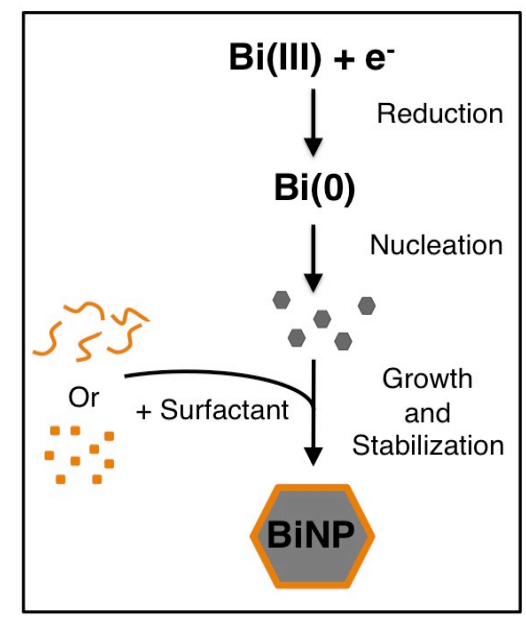

Scheme.1.1 General synthesis of BiNPs from a bismuth(III) precursor. A bismuth(III) cation is reduced via a reducing agent or in a reducing environment to form bismuth(0) atoms during the reduction phase. Bismuth(0) atoms then cluster to form nuclei (less than $1 \mathrm{~nm}$ in diameter) during the nucleation phase. Additional bismuth(0) atoms are added to these nuclei or multiple nuclei fuse, to form a nanoscale particle, and a polymer or small organic molecule surfactant then stabilizes the BiNP during the growth and stabilization phase.

\section{BiNP Preparations Using Bi[N(SiMe $\left.)_{2}\right]_{3}$ Precursor}

A number of bottom-up BiNP syntheses in apolar organic solvents have been reported to produce BiNPs; the best characterized and most widely applied of these preparations was developed by Buhro and coworkers.(60) In this 
preparation $\mathrm{BiCl}_{3}$ is reacted with $\mathrm{Na}\left[\mathrm{N}\left(\mathrm{SiMe}_{3}\right)_{2}\right]$ to obtain a soluble $\mathrm{Bi}\left[\mathrm{N}\left(\mathrm{SiMe}_{3}\right)_{2}\right]_{3}$ precursor material. The precursor, dissolved in tetrahydrofuran (THF), is heated under reflux with $\mathrm{Na}\left[\mathrm{N}\left(\mathrm{SiMe}_{3}\right)_{2}\right]$ at $180^{\circ} \mathrm{C}$ under an inert atmosphere. Poly(1-

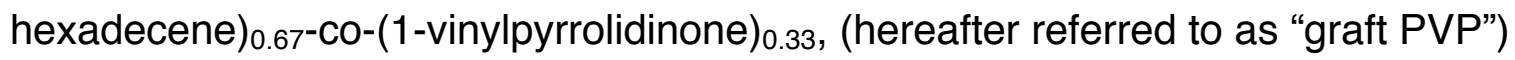
carried in 1,3-diisopropylbenzene (DIPB) is used as a surface stabilizer. By adjusting the $\mathrm{Na}\left[\mathrm{N}\left(\mathrm{SiMe}_{3}\right)_{2}\right]$ and/or polymer to bismuth ratio, spheres, wires, rods or hexagonal nanoplates were synthesized with good size and morphology uniformity. Shown in Figure 1.5 A-D are 4 representative images of nanoscale bismuth materials obtained by this method. The morphological effects of varying reagent concentrations, from an empirically optimized synthetic preparation for round nanoparticles, are explored as justification for each reagent and concentration used. Hexagonal nanoplates (Figure $1.5 \mathrm{~A}$ ) were obtained by decreasing the polymer concentration, nanowires (Figure 1.5 B) were obtained by decreasing the $\mathrm{Na}\left[\mathrm{N}\left(\mathrm{SiMe}_{3}\right)_{2}\right]$ concentration, and monodispersed spherical nanoparticles ranging from 3 to $30 \mathrm{~nm}$ (Figure $1.5 \mathrm{C}$ and $\mathrm{D}$ ) were obtained by carefully controlling the $\mathrm{Bi}\left[\mathrm{N}\left(\mathrm{SiMe}_{3}\right)_{2}\right]_{3}$ to $\mathrm{Na}\left[\mathrm{N}\left(\mathrm{SiMe}_{3}\right)_{2}\right]$ ratio (ratios of 1:2 up to 6:1 were used), with higher concentrations of $\mathrm{Na}\left[\mathrm{N}\left(\mathrm{SiMe}_{3}\right)_{2}\right]$ generally producing smaller particles, suggesting that the $\mathrm{Na}\left[\mathrm{N}\left(\mathrm{SiMe}_{3}\right)_{2}\right]$ is surface stabilizing the BiNPs in addition to the polymer. Larger particles (40 to $115 \mathrm{~nm}$ ) were obtained by increasing the $\mathrm{Bi}\left[\mathrm{N}\left(\mathrm{SiMe}_{3}\right)_{2}\right]_{3}$ concentration and decreasing the polymer and $\mathrm{Na}\left[\mathrm{N}\left(\mathrm{SiMe}_{3}\right)_{2}\right]$ concentration. The $\mathrm{Na}\left[\mathrm{N}\left(\mathrm{SiMe}_{3}\right)_{2}\right]$ and the graft PVP both affect the particle morphology, albeit these effects were tested empirically. The authors 
provide little information on the distinct role of each chemical reagent during the synthesis, however the role of $\mathrm{Na}\left[\mathrm{N}\left(\mathrm{SiMe}_{3}\right)_{2}\right]$ in the particle formation kinetics has been recently explored and will be discussed below. Interestingly Buhro and coworkers report that variation of the synthetic temperature from 160 to $210{ }^{\circ} \mathrm{C}$ does not appear to have a substantial influence on the size of the synthesized particles.
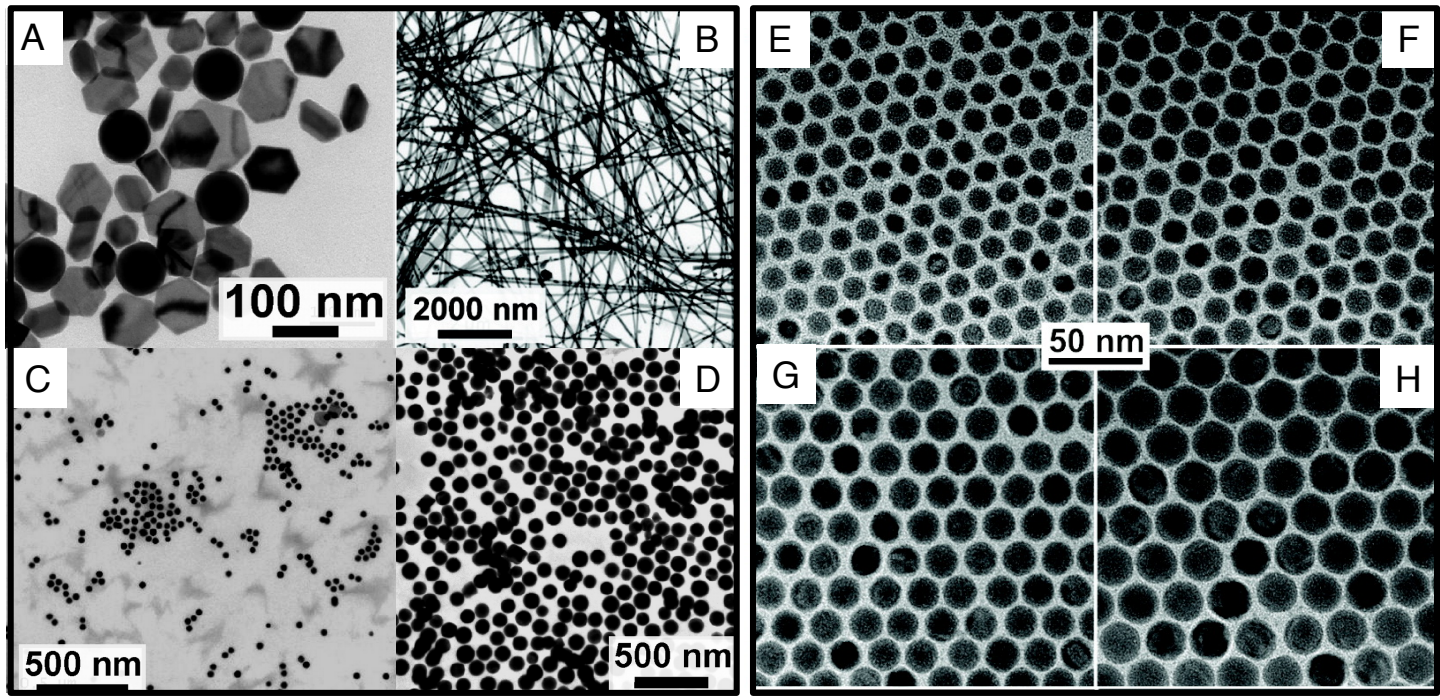

Figure 1.5 Representative TEM images of bismuth nanomaterials produced from a bismuth(III) silylamide precursor. Buhro and coworkers report synthesis of nanoplates (A), wires (B), and 3-30 nm relatively monodispersed nanoparticles (C and D) in THF using a specialty PVP polymer surfactant and $\mathrm{Na}\left[\mathrm{N}\left(\mathrm{SiMe}_{3}\right)_{2}\right]$ (from reference 60). Yarema et al. report the synthesis of monodisperse, 11$22 \mathrm{~nm}(\mathrm{E}-\mathrm{H})$ bismuth(0) nanoparticles in hexadecylamine with dissolved $\mathrm{Li}\left[\mathrm{N}\left(\mathrm{SiMe}_{3}\right)_{2}\right]$ that self organize into highly ordered superlattices (from reference $61)$. 
The temperature insensitivity of the preparation reported by Buhro and coworkers is in direct contrast with the work by Yarema et al., who noted that temperature is the important variable that controls nanoparticle size in their described synthesis, which also employed a $\mathrm{Bi}\left[\mathrm{N}\left(\mathrm{SiMe}_{3}\right)_{2}\right]_{3}$ precursor.(61) In this preparation hexadecylamine (HDA) serves as both solvent and surfactant, and no PVP is used. The bismuth precursor $\mathrm{Bi}\left[\mathrm{N}\left(\mathrm{SiMe}_{3}\right)_{2}\right]_{3}$, in addition to $\mathrm{Li}\left[\mathrm{N}\left(\mathrm{SiMe}_{3}\right)_{2}\right]$, was carried in toluene and injected into the hot HDA reaction solution. Particles were allowed to grow for 15 seconds, before the reaction was rapidly cooled to room temperature. By altering the synthetic temperature from 100 to $140^{\circ} \mathrm{C}$, the size of particles could be controlled from 11 to $22 \mathrm{~nm}$. Changing the duration of the reaction was shown to alter the size distribution, with a 15 second growth window producing the most monodisperse particles. The authors argued that incorporation of $\mathrm{Li}\left[\mathrm{N}\left(\mathrm{SiMe}_{3}\right)_{2}\right]$ in the reaction is essential for formation of morphology well controlled particles. Shown in Figure 1.6 are TEM images of two particle populations synthesized from $\mathrm{Bi}\left[\mathrm{N}\left(\mathrm{SiMe}_{3}\right)_{2}\right]_{3}$ in $\mathrm{HDA}$, in the absence (Figure 1.6 A) and in the presence (Figure 1.6 B) of $\mathrm{Li}\left[\mathrm{N}\left(\mathrm{SiMe}_{3}\right)_{2}\right]$. These images demonstrate the impact that the addition of $\mathrm{Li}\left[\mathrm{N}\left(\mathrm{SiMe}_{3}\right)_{2}\right]$ has on the morphology of the obtained nanoparticles, although particles were likely terminated predominantly by HDA solvent. 


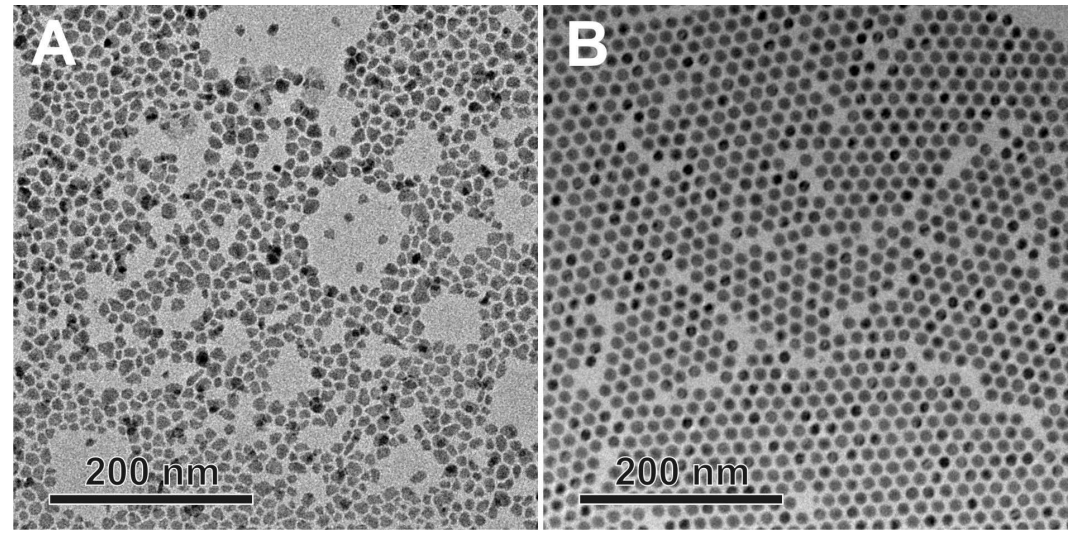

Figure 1.6 TEM images of BiNPs produced by the Yarema et al. method. Particles synthesized in $\mathrm{HDA}$ from $\mathrm{Bi}\left[\mathrm{N}\left(\mathrm{SiMe}_{3}\right)_{2}\right]_{3}$ in the absence $(A)$, and presence $(\mathrm{B})$, of $\mathrm{Li}\left[\mathrm{N}\left(\mathrm{SiMe}_{3}\right)_{2}\right]$. From reference (61) supporting information.

Buhro and co-workers have also performed a detailed kinetic analysis of their reaction with a focus on the mechanism.(62) They varied the $\mathrm{Bi}\left[\mathrm{N}\left(\mathrm{SiMe}_{3}\right)_{2}\right]_{3}$ to $\mathrm{Na}\left[\mathrm{N}\left(\mathrm{SiMe}_{3}\right)_{2}\right]$ ratio in the synthesis solution and removed aliquots for analysis over the duration of the reaction. By monitoring the sizes of the particles and the polydispersity of the populations at different time points during the reaction, they collected information about the reaction. Based on the number and size of particles observed in a sample information such as the time to maximum nucleation rate, duration of the nucleation period and the onset of Ostwald ripening, could be measured as a function of starting material concentration or other variables. Further analysis of this data included information such as a graph of the nucleation rate, which can be used to calculate the width of the nucleation function, namely the duration of the nucleation period, and relative standard deviation of particle sizes in the population. They showed a correlation between 
the nucleation window and the relative standard deviation of particle diameters that was expected from classical LaMer nucleation and growth kinetics. However, their analysis also revealed that after the onset of nucleation, Ostwald ripening and perhaps aggregative growth also occurred. Using the particle growth rated and kinetics data as a function of $\mathrm{Na}\left[\mathrm{N}\left(\mathrm{SiMe}_{3}\right)_{2}\right]$ concentration, the authors concluded that $\mathrm{Na}\left[\mathrm{N}\left(\mathrm{SiMe}_{3}\right)_{2}\right]$ serves as a facilitator for Ostwald-ripening and as a surface stabilizer that sterically hinders particle association and fusion.

The use of $\mathrm{Bi}\left[\mathrm{N}\left(\mathrm{SiMe}_{3}\right)_{2}\right]_{3}$ as a bismuth source for BiNP synthesis has produced some of the most morphologically uniform and size monodispersed nanoparticles reported in the literature. However, the light, temperature and air sensitivity of $\mathrm{Bi}\left[\mathrm{N}\left(\mathrm{SiMe}_{3}\right)_{2}\right]_{3}$ makes these syntheses difficult and cumbersome. To minimize the number of steps required to synthesize BiNPs and simplify their synthesis method, Buhro and co-workers later reported a modified method that produced BiNPs directly from a $\mathrm{BiCl}_{3 .}$ (63) In what they term a "shortcut" synthesis, a specific chemical addition sequence is key to the formation of particles. The addition of $\mathrm{BiCl}_{3}$ to the reaction vessel, followed by dry $\mathrm{THF}$, then the graft PVP, followed by $\mathrm{Na}\left[\mathrm{N}\left(\mathrm{SiMe}_{3}\right)_{2}\right]$, enabled them to produce particles similar in size and uniformity to the particle produced by their previously reported method, without first synthesizing and purifying a $\mathrm{Bi}\left[\mathrm{N}\left(\mathrm{SiMe}_{3}\right)_{2}\right]_{3}$ precursor. They again demonstrated a size control of the particles by adjusting the $\mathrm{Bi}\left[\mathrm{N}\left(\mathrm{SiMe}_{3}\right)_{2}\right]_{3}$ to $\mathrm{Na}\left[\mathrm{N}\left(\mathrm{SiMe}_{3}\right)_{2}\right]$ ratio. 
A study by Schulz et al., took advantage of the high reactivity and solubility of $\mathrm{Bi}\left[\mathrm{N}\left(\mathrm{SiMe}_{3}\right)_{2}\right]_{3}$ and focused on the synthesis of bismuth pseudocubes (a nanocrystal that appears morphologically cubic, but does not belong to the cubic crystal system, see Appendix A).(64) However, in this preparation they used this bismuth(III) source to first synthesize $\mathrm{Bi}_{2} \mathrm{Et}_{3}$ that was then thermally decomposed in THF with graft PVP, DIPB and $\mathrm{Na}\left[\mathrm{N}\left(\mathrm{SiMe}_{3}\right)_{2}\right]$. The solution was stirred at $50{ }^{\circ} \mathrm{C}$ for 4 hours, then at $170{ }^{\circ} \mathrm{C}$ for 12 hours to precipitate bismuth(0) pseudocubes out of solution. As with previous synthetic studies, the addition of the polymer and $\mathrm{Na}\left[\mathrm{N}\left(\mathrm{SiMe}_{3}\right)_{2}\right]$ were observed to affect the final morphology of the nanoparticles produced. Using a $\mathrm{Bi}_{2} \mathrm{Et}_{3}$ bismuth(III) precursor material is a novel synthesis choice because it is reactive enough at $50{ }^{\circ} \mathrm{C}$ to decompose and form highly faceted seeds that enable the growth of non-spherical BiNPs. The authors discussed the roles of PVP and $\mathrm{Na}\left[\mathrm{N}\left(\mathrm{SiMe}_{3}\right)_{2}\right]$ in controlling the morphology of the particles as they grew, and hypothesized that PVP should preferentially associate with specific bismuth(0) crystal faces to direct shape and that $\mathrm{Na}\left[\mathrm{N}\left(\mathrm{SiMe}_{3}\right)_{2}\right]$ serves to prevent aggregation of particles. However, they also note both the PVP and $\mathrm{Na}\left[\mathrm{N}\left(\mathrm{SiMe}_{3}\right)_{2}\right]$ are necessary in their synthesis to produce particles of a specific morphology, and thus conclude it is a kinetic rather than thermodynamic pathway directing particle synthesis. Given the studies by Schulz et al. and the conclusions drawn by Buhro and co-workers in their 3 papers, it is likely PVP and $\mathrm{Na}\left[\mathrm{N}\left(\mathrm{SiMe}_{3}\right)_{2}\right]$ are working together to direct particle morphology, but the role of each reagent is unclear. 
All of the methods described in this section are carried out under an anaerobic and anhydrous reaction environment and produce particles with a hydrophobic ligand display. While the reaction conditions and reagents used in these studies are not ideal for BiNPs that are to be employed in biological applications, the exquisite size and morphology control and detailed analysis of these particle preparations is useful for understanding BiNP formation kinetics and surface stabilization chemistry.

\section{Bismuth Nanoparticle Thiol Syntheses}

Bismuth(III) thiolate precursors have been reported for the synthesis of BiNPs, and while the solubility of a bismuth(III) thiolate $\left(\mathrm{Bi}(\mathrm{SR})_{3}\right)$ should be high in apolar solvents, the preferential formation of $\mathrm{Bi}_{2} \mathrm{~S}_{3}$ over bismuth(0) limits reaction conditions in which this type of precursor will form bismuth(0). Warren et al. described a BiNP synthesis that used light to catalyze the formation bismuth(0) in octadecene from a bismuth(III) decanethiol precursor.(65) This synthesis is a relatively simple and straight-forward process, whereby the thiolate ligands are oxidized to dithiols generating a ligand to metal charge transfer and a reducing environment capable of producing bismuth(0) particles. The process takes place in a sealed vessel exposed to ambient light over the course of two weeks. The authors called this a polythiol reaction and demonstrated its applicability in the synthesis of other elements, such as copper, lead and antimony nanoparticles. This method has a number of advantages such as a low cost reducing agent (the 
thiol), high dispersity of the metal coordinating ligand in apolar solvents that assists with solubility of inorganic atoms, and an ambient synthetic temperature. BiNPs produced by this method appear rhombohedral and form highly organized super-lattices when they are imaged by TEM (Figure 1.7 A).
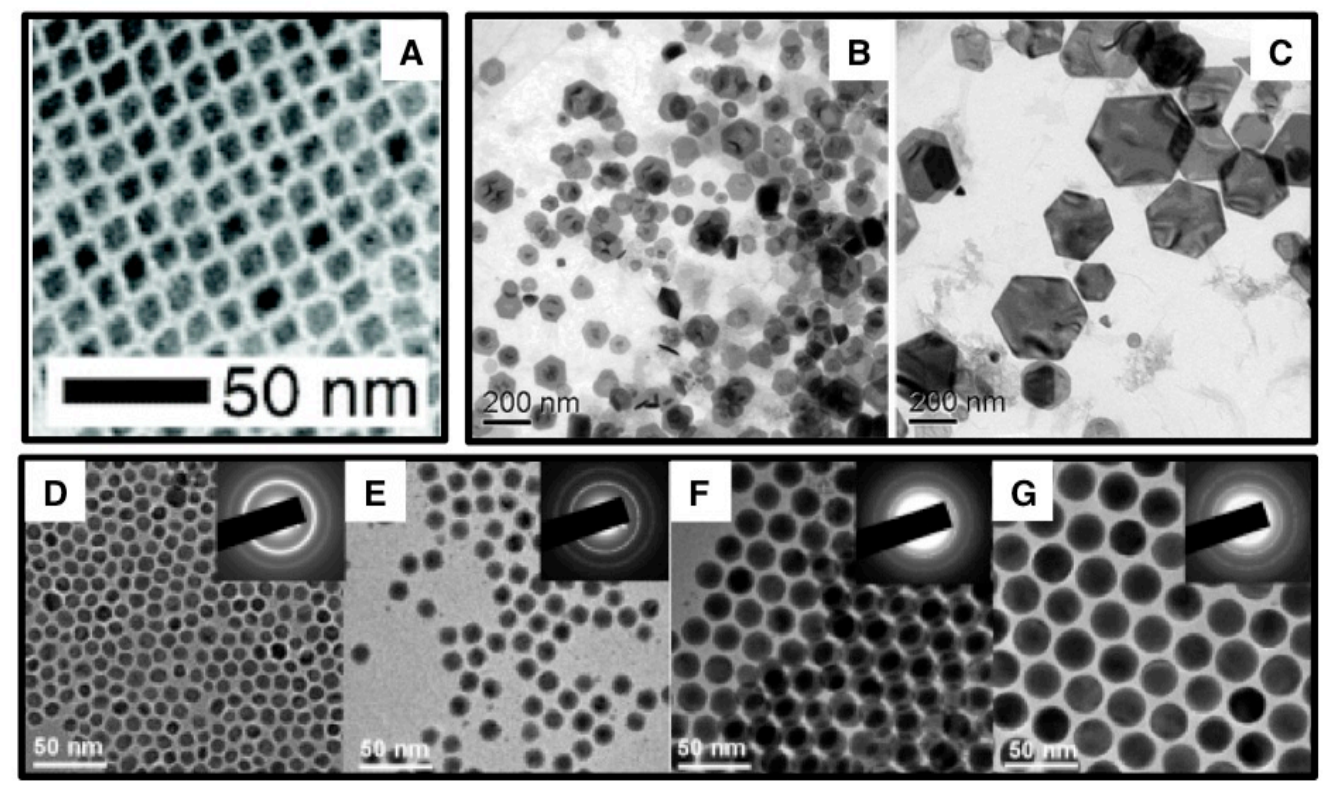

Figure 1.7 Representative TEM images of BiNPs produced from bismuth(III) thiolate precursors. Bismuth(III) decanethiol was suspended in octadecene and a light catalyzed formation of disulfide species generated bismuth( 0 ) particles (A, from reference 65.) Thermal decomposition of bismuth(III) dodecanethiolate in a sealed container produced bismuth $(0)$ particles at $120^{\circ} \mathrm{C}(\mathrm{B})$ or $135^{\circ} \mathrm{C}(\mathrm{C}$, from reference 66.) Bismuth(III) dodecanethiolate was reduced by tri-n-octylphosphine in octadecene to produce BiNPs of reported average sizes of $11 \mathrm{~nm}$ (D) $14 \mathrm{~nm}$ (E) $20 \mathrm{~nm}(\mathrm{~F})$ or $27 \mathrm{~nm}$ (G, from reference 67.)

A more recent preparation described by Chen et al.(66) also decomposed a bismuth(III) thiolate species to form bismuth(0) particles. In this work, 
bismuth(III) dodecanethiolate was synthesized, purified and sealed in a Pyrex tube, then heated for 10 hours to form bismuth(0) nanodisks. No additional solvent was used in the synthesis. The authors illustrated the effect that synthetic temperatures have on the particle sizes, with a $120{ }^{\circ} \mathrm{C}$ synthetic temperature producing relatively smaller particles (Figure 1.7 B) vs. a $135^{\circ} \mathrm{C}$ synthetic temperature (Figure 1.7 C.)

Son et al. (67) reported a synthesis that used bismuth(III) dodecanethiolate reduced by tri-n-octylphosphine (TOP) in octadecene to produce BiNPs 6 to $17 \mathrm{~nm}$ in size (Figure $1.7 \mathrm{D}-\mathrm{G}$ ). The temperature range (55 to $80^{\circ} \mathrm{C}$ ), growth time (1.5 to 30 minutes) and the TOP concentration and injection temperature $\left(20\right.$ to $70{ }^{\circ} \mathrm{C}$ ) were varied to produce different sized particles. Because the focus of the study was on producing particles for thermoelectric measurements, synthetic details and mechanisms were not fully explored. Increasing the synthetic temperature (from $55^{\circ} \mathrm{C}$ to $65^{\circ} \mathrm{C}$ ) produced particles from 6 to $11 \mathrm{~nm}$, and increasing TOPO concentration and growth time (at $70{ }^{\circ} \mathrm{C}$ to $80{ }^{\circ} \mathrm{C}$ ), in addition to increasing the temperature of the TOPO addition, produced particles 14 to $27 \mathrm{~nm}$ in size. Shown in Figure 1.7 D-G are particles synthesized by this method. It should be noted that small particle nuclei are evident in some of the TEM images (particularly in Figure 1.7 E) suggesting a heterogeneity that is apparently not included in the reported population distribution. 


\section{Organic-phase BiNP Syntheses Using Bismuth(III) Salts}

The use of the polar organic solvent $\mathrm{N}, \mathrm{N}$-dimethylformamide (DMF) for BiNP synthesis is popular, and a few of these preparations, which are generally performed under anaerobic conditions, will be described here. These preparations use commercially available bismuth(III) salts, such as $\mathrm{BiCl}_{3}$ or $\mathrm{Bi}\left(\mathrm{NO}_{3}\right)_{3}$, and $\mathrm{NaBH}_{4}$ as a reducing agent. Additionally these preparations use PVP as a surface stabilizer, most likely, as discussed above, because of the high electron density donation afforded by the amide group. Wang et al., report a preparations that used $\mathrm{BiCl}_{3}$ as the starting bismuth(III) source, and described the effect that the ratio of PVP to bismuth(III) has on the size and extent of aggregation of BiNPs produced by this reaction (Figure 1.8 A and $\mathrm{B}$ ). When particles were synthesized with a PVP to bismuth(III) ratio of less than 1:1 (Figure 1.8 A) the particles appeared highly aggregated. Particles synthesized with a PVP to bismuth(III) ratio of $5: 1$ are smaller and less aggregated, suggesting a greater separation of the nanocrystals in solution when the concentration of surfactant is higher (Figure 1.8 B). They further characterized these particles and described the formation of the PVP-bismuth bond by measuring a shift in the FTIR absorbance of the carbonyl group in PVP. The $\mathrm{C}=\mathrm{O}$ bond in neat PVP has a bond absorbance at $1675 \mathrm{~cm}^{-1}$, and when associated with a bismuth(III) cation in solution, this bond absorbance shifts to $1588 \mathrm{~cm}^{-1}$. This can be explained by an increase in electron density around the oxygen atom, and consequently a decrease in the electron density in the $\mathrm{C}=\mathrm{O}$ bond. This decrease in electron 
density at the carbonyl double bond leads to a lower energy wavelength absorbance. When PVP surface stabilizes BiNPs, this absorbance wavelength shifts to $1659 \mathrm{~cm}^{-1}$. These observations can be explained by the formation of a coordinative PVP-bismuth(III) bond when the polymer is associated with the bismuth(III) cation. After reduction of the bismuth(III) ions, the PVP retains a coordinative bonding interaction with the bismuth $(0)$ nanoparticle surface. This observation suggests that relative to neat PVP, the carbonyl group is mildly electron donating to the bismuth(0) nanoaparticle surface, and strongly electron donating to the bismuth(III) cation in solution.

Interestingly DMF also contains an amide group, and while the PVP polymer has been demonstrated to be effective for colloidal stabilization of BiNPs in solution, it seems likely that some DMF also surface stabilizes and coordinates to the particle surfaces. Su and co-workers have worked with BiNPs produced by reduction of $\mathrm{Bi}\left(\mathrm{NO}_{3}\right)_{3}$ by $\mathrm{NaBH}_{4}$ in DMF with PVP surface stabilization (Figure 1.8 C and D). Transfer of these particles from an organic solvent to aqueous BiNPs has been demonstrated, but because the synthesis was carried out in a highly coordinating solvent, namely DMF, it is likely that traces of the solvent are still present on the particle surfaces. This contamination could yield cytotoxic BiNPs that are unrelated to bismuth(0) toxicity. 

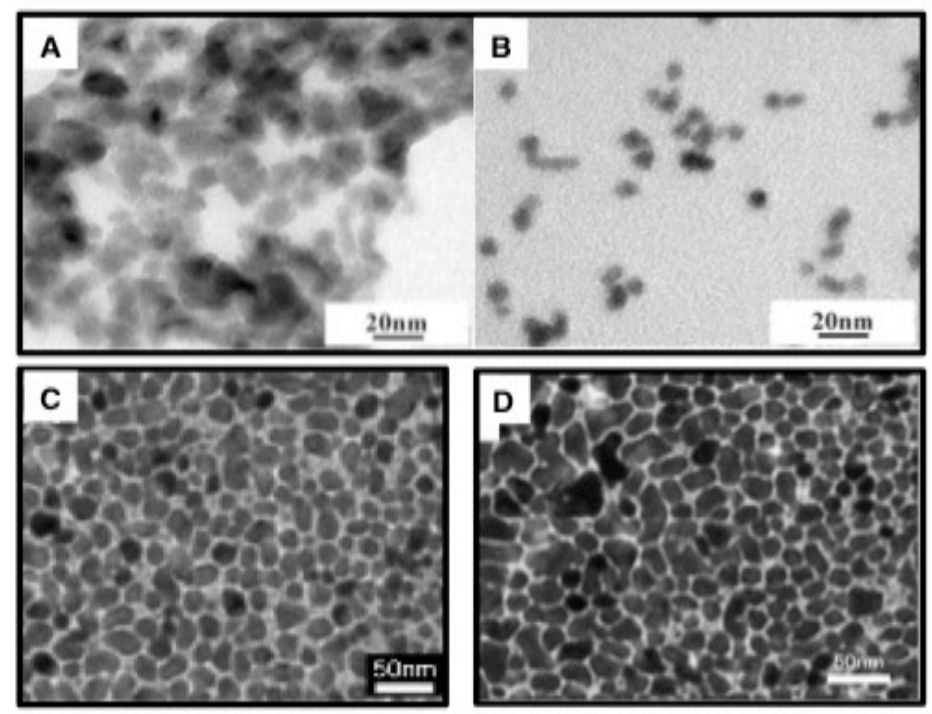

Figure 1.8 BiNPs produced by $\mathrm{NaBH}_{4}$ reduction of a bismuth(III) salt in DMF in the presence of PVP. The ratio of bismuth(III) to PVP affects the particles size and extent of aggregation, as illustrated by particles synthesized using a PVP to bismuth(III) ratio of 0.15:1 (A) and 5:1 (B, from reference 68.) BiNPs synthesized with a PVP to bismuth(III) ratio of $5: 1$ (C and D) were phase transferred to aqueous solutions and used in cell detection and biocompatibility experiments. From references (69) and (70).

A biocompatibility assessment of these BiNPs, which were used to target and detect specific cell types by X-ray fluorescence spectroscopy, reported a low level of cytotoxicity in one study,(69) but the same group also reported some cytotoxicity of similar BiNPs that depended on the BiNP surface coating.(70) The particles used in the these studies were all synthesized in DMF, and were then further reacted in toluene with polyethylene glycol (PEG) or other surfactants to give water soluble particles with a range of surface charges and functionalities. Specifically this study found that capping BiNPs with a silica shell, or with PEG, 
produced particles with a slightly lower toxicity in vitro, relative to the unmodified particles. Given the difficulty of removing surface bound solvents from high surface area materials and purification of particles from toxic solvents or reagents, aqueous and/or biologically compatible solvents, such as diols, were primarily explored for the synthesis of BiNPs in this document.

\section{Polyol Synthesis of BiNPs}

The polyol synthesis is an ill-defined chemical reaction that has been used to produce a wide variety of inorganic nanoparticles. In short, a poly-alcohol solvent is heated in the presence of a metal cation, that is then reduced to an inorganic nanoparticle.(71) Syntheses of BiNPs have been reported by the polyol process, $(59,72-77)$ where a bismuth(III) or (V) precursor is heated to a specified temperature to produce bismuth(0) particles. Additional surfactants can effect morphology control of the particles, and will be discussed. The preparation of BiNPs in a biocompatible poly-alcohol solvent is a potential non-aqueous route to produce medically applicable BiNPs for use as XCAs, provided that the surface is adequately protected against oxidative decomposition in aqueous solutions. 


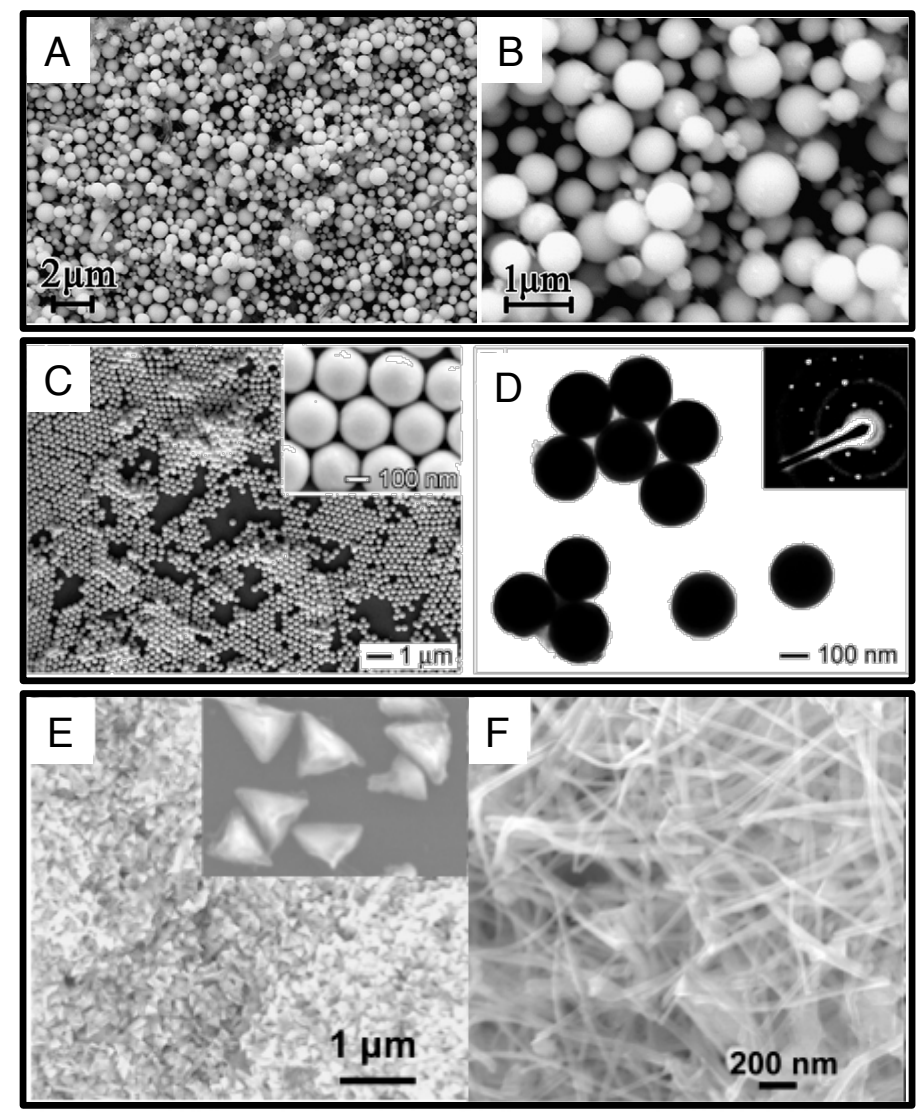

Figure 1.9. Representative electron microscopy images of bismuth nanomaterials produced by the polyol synthesis. All particle syntheses were carried out in ethylene glycol heated at reflux. Bismuth(III) citrate was reacted with urea and PVP (A and B, from reference 78.) Bismuth(III) acetate was reacted with PVP (C and D, from reference 59.) Bismuth(III) chloride was reacted PVP with two different (unspecified) concentrations of $\mathrm{NaOH}$ to produce nonspherical particles ( $E$ and $F$, from reference 73 .)

Polyol syntheses are most commonly carried out in ethylene glycol, and three preparations that used ethylene glycol as a solvent and PVP as a surfactant will be discussed here. Cheng et al. reported the used of bismuth(III) citrate and urea, which were heated in ethylene glycol to reflux in the presence of 
PVP under atmospheric conditions.(78) Figure 1.9 A and B show SEM images of the particles obtained from this synthesis, which are clearly round and relatively size polydisperse. The addition of cetyltrimethylammonium bromide or PEG to the reaction produced less well-formed particles that have a higher size polydispersity (not shown). Wang and Xia reported the decomposition of bismuth(III) acetate in ethylene glycol (heated under reflux) in the presence of PVP.(59) Particles produced by this method are shown in Figure $1.9 \mathrm{C}$ and $\mathrm{D}$, and are a second part of the same study discussed in section 1.6 .1 of this document. The authors showed a correlation between the size of the particles produced relative to the concentration of bismuth(III) acetate used during the synthesis. As this particular study was focused on the formation of low melting point elements, specifically the formation of bismuth(0) from the bismuth(III) acetate precursor, they additionally noted the effect that stirring rate has on the final particle size.

A polyol process using a $\mathrm{BiCl}_{3}$ starting material and PVP in ethylene glycol with and without the addition of $\mathrm{NaOH}$, has been published by two different groups, who reported the production of primarily bismuth(0) nanowires. In both studies spherical nanoparticles were occasionally noted, and the role of $\mathrm{NaOH}$ appears to be important for shape control.(73, 79) Specifically, addition of hydroxide appears to produce a higher yield of wires in the final nanomaterial product. Shown are representative examples of nanoscale bismuth(0) triangles (Figure $1.9 \mathrm{E}$ ) synthesized with lower additive amounts of $\mathrm{NaOH}$, relative to the 
amounts used to obtain nanowires (Figure 1.9. F) in the reaction mixture.(73) The authors argued that the role of $\mathrm{NaOH}$ is to enable the formation a $\mathrm{BiO}_{2}{ }^{-}$ intermediate bismuth(III) species that dictates the rate of nanomaterial formation and thus the shape of the final nanomaterial obtained.
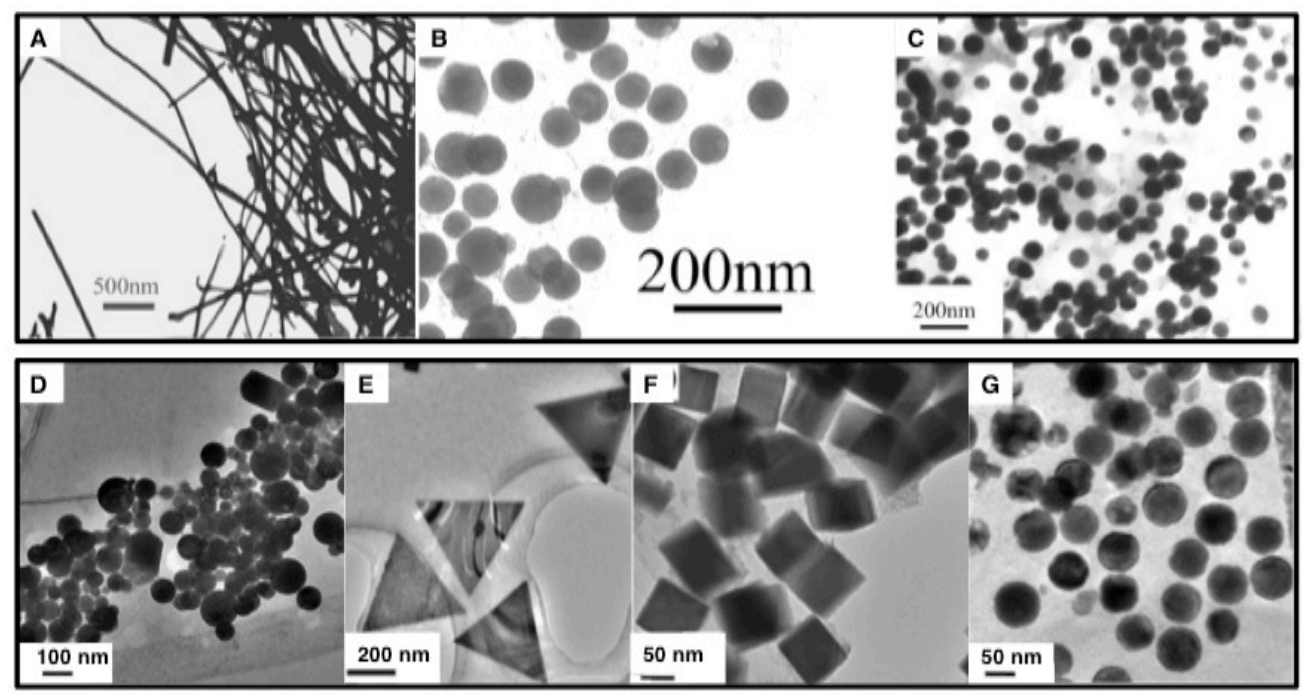

Figure 1.10 TEM images of products of polyol synthesis using a $\mathrm{NaBiO}_{3}$ precursor. The bismuth $(\mathrm{V})$ precursor is heated in a sealed vessel with ethylene glycol and PVP (A) or with acetone at a ethylene glycol to acetone ratio of 1:2 (B) or 1:4 (C, from reference 74.) Shape control of BiNPs was achieved in a similar synthesis by altering the molar ratio of PVP to bismuth from 0:1 (D), 0.8:1 (E), 1.6:1 $(F)$ to $5: 1$ (G, from reference 77 .)

The use of $\mathrm{NaBiO}_{3}$ precursor that is thermally decomposed and reduced to bismuth $(0)$ in the presence of a surfactant has been explored in two publications, which are unique because they are the only two preparations that use a bismuth(V) precursor. In the earlier work by Wang and co-workers, $\mathrm{NaBiO}_{3}$ was suspended in ethylene glycol in a sealed autoclave vessel with PVP or 
acetone, that was then degassed and heated to 180 to $200{ }^{\circ} \mathrm{C}$ for 24 hours.(74) Bismuth(0) nanowires were obtained in the presence of PVP, and bismuth(0) spheres were the predominant morphology observed when acetone was present in place of PVP. Figure 1.10 A shows a representative TEM image of nanowires obtained by this method when PVP was used as a surfactant. Figure $1.10 \mathrm{~B}$ and C show spherical particles obtained by this method when acetone was present in solution at an ethylene glycol to acetone ratio of $1: 2$ and 1:4, respectively.

In the latter work by Wang and co-workers, the ratio of bismuth(V) to PVP was explored as a way to morphology control the resultant bismuth(0) materials.(77) Using a similar synthetic protocol to their previous work, by controlling the bismuth to PVP ratio, the authors were able to synthesize spheres (Figure 1.10 D), triangles (Figure 1.10 E) and cubes (Figure 1.10 F). Interestingly, wires were reported to form in this study only when an iron(III) species was added to the synthesis, suggesting that a trace contamination of a transition metal in the previous work when wires were regularly produced. The authors postulated that the role of iron(III) is to decrease the rate of bismuth(0) formation, thus limiting the nucleation rate and increasing the growth rate, which should give preferential rise to bismuth(0) nanowires. 


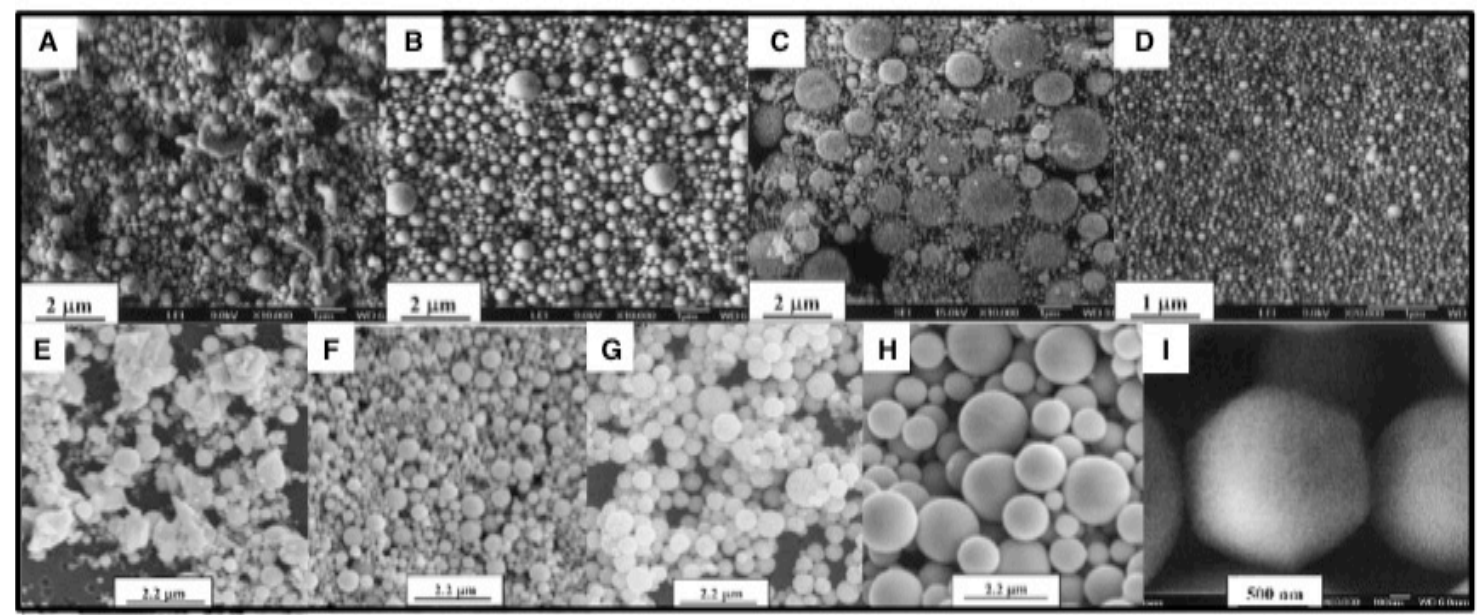

Figure 1.11. Electron microscopy images of bismuth(0) particles produced by the polyol method, from reference (72). Particles were synthesized by boiling bismuth(III) hydroxide in 1,3 propane diol (A), diethylene glycol (B), glycerol (C), or tetraethylene glycol (D). Particles were also synthesized in propylene glycol by reduction of bismuth(III) acetate (E), bismuth(III) nitrate $(\mathrm{F})$, or bismuth(III) subcarbonate $(G)$. Additionally particles were synthesized in mixture of propylene glycol and triethanol amine that was used to reduce bismuth(III)hydroxide $(\mathrm{H}$ and I, all from reference 72.)

A systematic study of the polyol synthesis for the formation of bismuth(0) particles by Goia et al. used a variety of diol solvents and reported a wide range of particle sizes produced.(72) The authors surveyed polyalcohol solvents, such as propylene glycol, 1,3-propanediol, diethylene glycol, glycerol and tetraethylene glycol to commonly used ethylene glycol. They also surveyed bismuth(III) sources including bismuth(III) nitrate, bismuth(III) acetate, bismuth(III) oxide and bismuth(III) carbonate as bismuth precursor materials. The authors suggested that the counter-ion and acidity of the bismuth(III) salt may affect particle 
formation kinetics, but that the solvent boiling point was the most important factor for particle morphology control. Interestingly the authors concluded that a minimum synthesis temperature of $220{ }^{\circ} \mathrm{C}$ is necessary for reduction of bismuth(III) acetate to bismuth(0), which was substantially higher than the synthetic temperature reported by Wang and Xia in their polyol synthesis.(59) However, concentration, Goia et al. used a 200 mM bismuth concentration, Wang and Xia used 10 to $70 \mathrm{mM}$ concentration, or the addition of PVP to the reaction solution, may be responsible for this disparity.

The particle morphologies observed in this study were generally spherical, albeit the particle sizes obtained under different synthetic conditions were highly variable. Figure 1.11 shows some of the particle populations obtained in this particular study and were chosen to illustrate size ranges and morphology distributions. When bismuth(III) hydroxide was heated to reflux in 1,3-propanediol (Figure 1.11 A), diethylene glycol (Figure 1.11 B), glycerol (Figure 1.11 C) or tetraethylene glycol (Figure 1.11 D) particles appear generally spherical and very size polydisperse. Tetraethylene glycol appears to produce the smaller particle population, and glycerol contains a particle population with some of the largest particles observed. Propylene glycol was used to produce bismuth(0) particles from bismuth(III) acetate (Figure 1.11 E) bismuth(III) nitrate (Figure 1.11 F) and bismuth(III) subcarbonate (Figure $1.11 \mathrm{G}$ ), and the morphologies observed were variable depending on the bismuth (III) counter ion used. The most well morphology controlled particles were produced from bismuth(III) hydroxide in a 
mixture of diethylene glycol and triethanolamine (Figure $1.11 \mathrm{H}$ and I). This illustrates the versatility of the polyol reaction for the production of variable bismuth(0) materials and is highly promising for the synthesis of biologically compatible BiNPs. However aqueous stability of these particles, in addition to increasing the homogeneity of particle sizes, would need to be addressed for further development of BiNPs for biological application.

\section{Aqueous Syntheses of BiNPs}

Reported aqueous preparations of BiNPs are rare and the morphologies of BiNPs produced in aqueous solutions are in general substantially less uniform than particles synthesized in organic solvents (see Section 3.2 for further discussion.) An early aqueous phase synthesis of BiNPs was accomplished using a reverse micelle method. In this preparation, dioctyl sulfosuccinate sodium salt in isooctane was used to form reverse micelles around aqueous $\mathrm{BiOClO}_{4}$, which were then reacted with reverse micelles containing $\mathrm{NaBH}_{4}$ to produce elemental BiNPs.(80) The resultant particles were morphologically heterogeneous and generally smaller than $10 \mathrm{~nm}$. However, the use of micelles is a logical approach for nanomaterial synthesis, as the micelles provide a confined reaction volume for particles synthesis and should prevent ripening or fusing of the produced particles. Additionally the micelles should prevent other solvent or environmental reactions with the growing nanoparticles. 

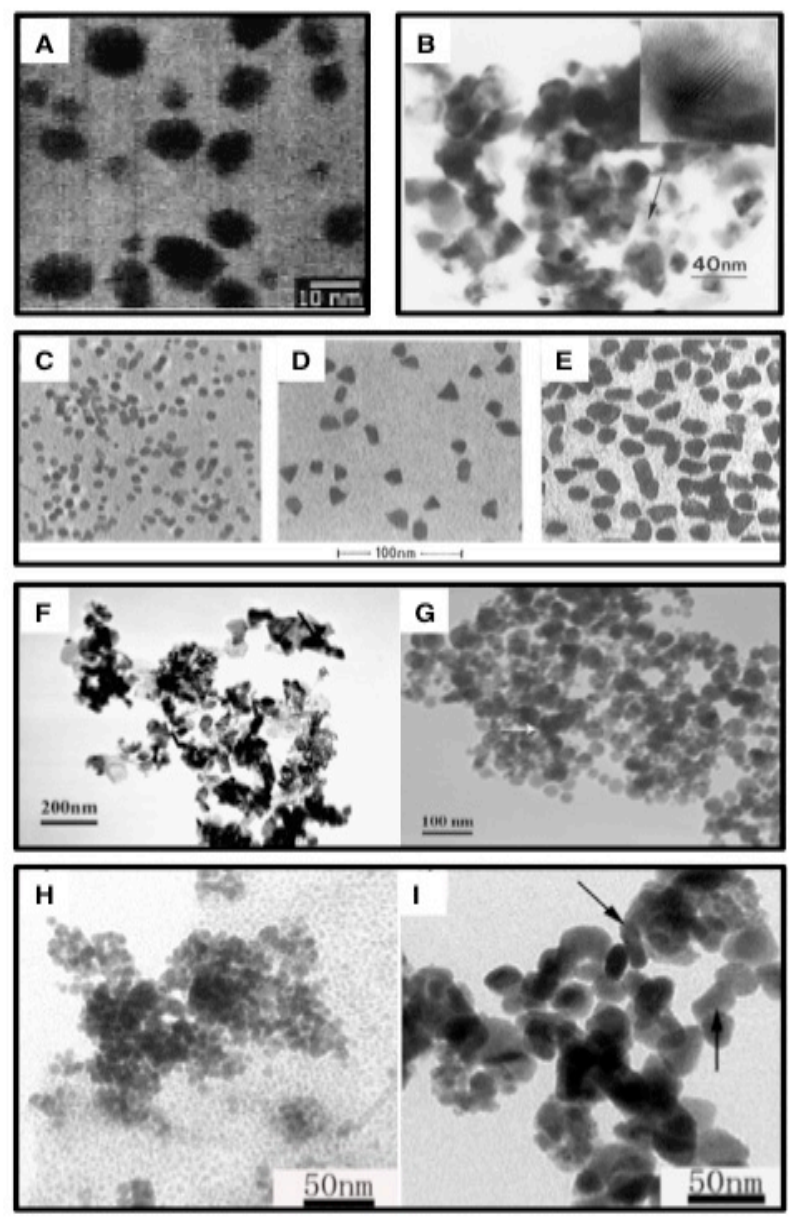

Figure 1.12 TEM images of BiNPs synthesized in aqueous solvents. Particles synthesized from $\mathrm{BiClO}_{4}$ and $\mathrm{NaBH}_{4}$ in reverse micelles (A, from reference 80.) BiNPs produced by an emulsion process from bismuth(III) citrate and $\mathrm{NaBH}_{4}(\mathrm{~B}$, from reference 81 and 82 .) $\mathrm{BiOClO}_{4}$ was reduced in water by a $\gamma$ ray source in a $70 \%$ (C) or $10 \%$ (D) polyacrylamide solution, or in a $10 \%$ polyacrylamide solution with the addition of cadmium(II) ions ( $E$, from reference 83.) BiNPs produced from aqueous $\mathrm{BiCl}_{3}$ reduced by hydrazine hydrate in the absence $(\mathrm{F})$ and presence $(G)$ of sodium oleate, from reference (84). BiNPS produced from bismuth(III) nitrate suspended by tartaric acid in a basic solution, and reduced by sodium-hypophosphite over $5(\mathrm{H})$ and $10(\mathrm{I})$ hours, from reference (85). 
Fang et al. synthesized bismuth(0) particles in aqueous solution using a bismuth(III) citrate precursor reduced by $\mathrm{NaBH}_{4}$ in an emulsion process. $(81,82)$ Poly(oxyethylene $)_{9}$ nonyl phenol ether and poly(oxythylene) $)_{5}$ nonyl phenol ether were used as amphiphilic polymers for micellular formation in petroleum ether, with an aqueous solution of ammonium citrate. The particles were subsequently washed and suspended in PVP and ethanol to prevent surface oxidation during analysis. The TEM images of the particles show polycrystalline aggregates with rough surfaces and inhomogeneous morphologies, as well as a high polydispersity in nanocrystal size (Figure $1.12 \mathrm{~B}$ ). Another preparation by the same group, using again the microemulsion process, employed a $\mathrm{Bi}\left(\mathrm{NO}_{3}\right)_{3}$ starting source and $\mathrm{NaBH}_{4}$ as a reducing agent, albeit with methyl methacrylate or hydroxyethyl grafted methyl methacrylate as the amphiphile to form the micro emulsion. This preparation produced similarly aggregated and amorphous bismuth(0) materials (image not shown.)(86)

The most morphologically well controlled aqueous preparation of BiNPs published to date was accomplished by pulse radiolysis reduction of $\mathrm{BiOClO}_{4}$ in water with 2-propanol surfactant using a $\gamma$ radiation source and either polyethyleneimine or polyacrylamide as a capping agent.(83) Briefly, a ${ }^{60} \mathrm{Co}$ source provided a $5.2 \times 10^{4} \mathrm{rad} / \mathrm{h}$ dose to a $100 \mathrm{~mL}$ aqueous solution of the bismuth(III) source. Using a radiation pulse, the authors argued that the $\gamma$ radiation produced a hydrated electron, an $\mathrm{H}$ atom and a hydroxyl radical, and that the function of 2-propanol was to scavenge the hydroxyl radical thus 
producing a reducing environment via an organic radical. The authors showed that a change in the final particle size and morphology was possible by adjusting the amount of the polymer surfactant. Particles produced in $70 \%$ polyacrylamide solutions were smaller (Figure $1.12 \mathrm{C}$ ) relative to particles produced in $10 \%$ polymer surfactant (Figure $1.12 \mathrm{D}$ ). The addition of $\mathrm{Cd}\left(\mathrm{ClO}_{4}\right)_{2}$ to the reaction solution was used to probe the mechanism of the radiation induced reduction. The $\gamma$ radiation does not reduce cadmium(II) when a bismuth(III) source is not present, but colloidal cadmium( 0$)$ is produced when colloidal bismuth $(0)$ is present as a catalyst. The authors suggest that in this case, the $\gamma$ radiation is unable to reduce the cadmium(II) to cadmium(0) without a bismuth intermediate either stabilizing a cadmium(I) intermediate or by direct transfer of charge from bismuth(0) to cadmium(II). The bismuth(0) particles produced in $10 \%$ polymer surfactant solution in the presence of cadmium(II) (Figure $1.12 \mathrm{E}$ ) are qualitatively similar to particles produced in the absence of cadmium(II) (Figure $1.12 \mathrm{D})$, and interestingly if the polymer concentration is decreased, cadmium(0) can be deposited on the bismuth(0) particle surface. While the use of a radiation source is impractical for the large-scale synthesis of BiNPs in an unequipped laboratory, the reducing source is novel and the used of methyl methacrylate as a surface passivating polymer is interesting because of the aqueous stability it imparts on the BiNPs surface.

Another aqueous BiNP synthesis was accomplished at $80^{\circ} \mathrm{C}$, and used a $\mathrm{BiCl}_{3}$ as a starting reagent, sodium oleate as a surfactant and hydrazine hydrate 
as a reducing agent. The authors argued that the reducing agent also helped to buffer the $\mathrm{pH}$ during synthesis to promote oxidative stability of the BiNPs in water.(84) Oleate, as a reactant in an aqueous synthesis, should phase isolate the growing BiNPs, which may explain the hydrolytic stability, but it also promotes aggregation and agglomeration of particles in water. However, in the absence of the oleate surfactant, bismuth(0) material is obtained, but is highly surface irregular and inhomogenous (Figure $1.12 \mathrm{~F}$ ) relative to the bismuth $(0)$ particles obtained in the presence of oleate (Figure $1.12 \mathrm{G}$ ). Interestingly, this synthesis took place at $80{ }^{\circ} \mathrm{C}$, and the authors noted that above $90{ }^{\circ} \mathrm{C}$ the reaction took place so quickly that bulk bismuth(0) was formed, as evidenced by the observation of a highly aggregated and metallic material. Alternately, below $60{ }^{\circ} \mathrm{C}$, only the formation of a grey precipitate is observed, which is most likely a bismuth oxide species. They noted that the morphology of the particles is independent of oleate concentration, which is unexpected given the dramatic morphology change observed when no oleate was present.

An aqueous BiNP preparation that used a novel method to suspend bismuth(III) cations, used tartaric acid in a basic solution as a ligand to promote solubility for the cation.(85) In short, $\mathrm{Bi}\left(\mathrm{NO}_{3}\right)_{3}$ was reacted with tartaric acid in an acidic solution, and sodium hydroxide was added to form a clear, suspended bismuth(III) aqueous precursor. The solution was brought to $90^{\circ} \mathrm{C}$, and sodium oleate, followed by sodium-hypophosphite, was added and the reaction was allowed to stir for 5-10 hours. The authors noted that in the absence of tartate, 
under basic conditions, a white bismuth(III) oxide was formed that was difficult to further reduce to bismuth(0). The authors also noted that smaller particles were obtained when the reaction proceeded for 5 hours (Figure $1.12 \mathrm{H}$ ) and that relatively large, more rod shaped particles were obtained during the 10 hour reaction time (Figure $1.12 \mathrm{I}$ ). Varying the tartaric acid concentration had little effect on the final particle morphology, but decreasing the concentration of $\mathrm{NaOH}$ induced the formation of strings of disorganized particles. Increasing the $\mathrm{NaOH}$ concentration led to an increase in particle polydispersity, and larger particles or aggregates were observed. The authors speculated that the concentration of $\mathrm{NaOH}$ affected the bismuth(III) tartate complex and thus the bismuth(III) reduction rate.

\subsection{Scope of this Work}

The scope of this work is to further develop synthetic strategies for the formation of bismuth(0) nano and micon scale materials to be used in medical applications. BiNPs that are highly X-ray opaque and imputed to contain no toxic organic molecules is the primary synthetic aim of Chapters 2 and 3. As BiNP synthetic preparations commonly contain toxic organic solvents, are not oxidatively stable in aqueous solutions or are poorly morphology controlled, novel synthetic routes were undertaken. Chapter 2 describes an aqueous BiNP synthesis that uses a previously undescribed BiNP surfactant, dextran, in a pH controlled solution. Chapter 3 describes a BiNP synthetic strategy that takes 
advantage of the solubility of bismuth(III) cations in 1,2-propane diol and uses a glucose surfactant to stabilize particles in aqueous solutions. Particles are shown to be highly X-ray opaque as characterized by a quantitative CT study, and preliminary cytotoxicology results suggest that particles will have cell type specific responses.

Chapter 4 of this dissertation describes the use of an uncharacterized bismuth(III) iodide precursor suspended in hexadecylamine to produce BiNPs by an orgaoamine reaction. Particles are putatively amine terminated, and may find application in BiNP ligand exchange studies.

Chapter 5 of this dissertation describes an inexpensive synthesis of a bismuth(0) microparticles for use in highly X-ray opaque composite materials. These materials are expected to find application in medical devices for the tagging, locating and tracking of X-ray transparent medical equipment.

\section{References:}

(1) The new light: discovery and introduction of the X-ray. American Journal of Roentgenology 1995, 165, 1041-1045.

(2) Brenner DJ, H. H. Radiation exposure from medical imaging: Time to regulate? Journal of the American Medical Association 2010,304, 208-209.

(3) http://medical-dictionary.thefreedictionary.com/barium+enema (accessed March 7, 2013). 
(4) National Kidney and Urologic Diseases Information Clearinghouse. http://kidney.niddk.nih.gov/kudiseases/pubs/imagingut/ (accessed August 9, 2011).

(5) Bourin, P. M.; Jolliet, P.; Ballereau, F. An Overview of the Clinical Pharmacokinetics of X-Ray Contrast Media. Clinical-Pharmacokinetics 1997, 32, 180-193.

(6) Physiology or Medicine 1979 - Press Release. http://www.nobelprize.org/nobel_prizes/medicine/laureates/1979/press.html (accessed March 14, 2013).

(7) Goodman DM Initiatives focus on limiting radiation exposure to patients during ct scans. Journal of the American Medical Association 2013, 309, 647-648.

(8) US Department of Commerce, N. NIST: X-Ray Mass Attenuation Coefficients. http://www.nist.gov/pml/data/xraycoef/index.cfm (accessed August 9, 2011).

(9) Cormode, D. P. Potential of multi-color computed tomography for advanced disease characterization. MEDICAMUNDI 2011, 55.

(10) Fromm, K. M. Barium bright and heavyNature Chemistry 2013, 5, 146-146.

(11) Heiken, J. P.; Brink, J. A.; McClennan, B. L.; Sagel, S. S.; Crowe, T. M.; Gaines, M. V. Dynamic incremental CT: effect of volume and concentration of contrast material and patient weight on hepatic enhancement. Radiology 1995, 195, 353-357. 
(12) Newington, I. M.; Humphries, G.; Lasbistes, N.; Morisson-Iveson, V.; Nairne, J.; Passmore, J.; Thanning, M.; Wistrand, L.-G.; Wynn, D. The synthesis and evaluation of trimeric X-ray contrast agents. Tetrahedron Letters 2011, 52, 3065-3067.

(13) Jean-Marc, I.; Emmanuelle, P.; Philippe, P.; Claire, C. Allergy-like reactions to iodinated contrast agents. A critical analysis. Fundamental \& Clinical Pharmacology 2005, 19, 263-281.

(14) Namasivayam, S.; Kalra, M. K.; Torres, W. E.; Small, W. C. Adverse reactions to intravenous iodinated contrast media: a primer for radiologists. Emergeny Radiology 2006, 12, 210-215.

(15) Spring, D. B.; Bettmann, M. A.; Barkan, H. E. Nonfatal adverse reactions to iodinated contrast media: spontaneous reporting to the U.S. Food and Drug Administration, 1978-1994. Radiology 1997, 204, 325-332.

(16) Persson, P. B.; Hansell, P.; Liss, P. Pathophysiology of contrast mediuminduced nephropathy. Kidney International 2005, 68, 14-22.

(17) Solomon, $R$. The role of osmolality in the incidence of contrast-induced nephropathy: A systematic review of angiographic contrast media in high risk patients. Kidney International 2005, 68, 2256-2263.

(18) Hallouard, F.; Briançon, S.; Anton, N.; Li, X.; Vandamme, T.; Fessi, H. lodinated nano-emulsions as contrast agents for preclinical X-ray imaging: Impact of the free surfactants on the pharmacokinetics. European Journal of Pharmaceutics and Biopharmaceutics. 
(19) Hallouard, F.; Anton, N.; Choquet, P.; Constantinesco, A.; Vandamme, T. lodinated blood pool contrast media for preclinical X-ray imaging applications - A review. Biomaterials 2010, 31, 6249-6268.

(20) Nune, S. K.; Gunda, P.; Thallapally, P. K.; Lin, Y.-Y.; Laird Forrest, M.; Berkland, C. J. Nanoparticles for biomedical imaging. Expert Opinion Drug Delivery 2009, 6, 1175-1194.

(21) Cho, E. C.; Glaus, C.; Chen, J.; Welch, M. J.; Xia, Y. Inorganic nanoparticle-based contrast agents for molecular imaging. Trends in Molecular Medicine 2010, 16, 561-573.

(22) Lusic, H.; Grinstaff, M. W. X-ray-Computed Tomography Contrast Agents. Chemical Reviews. 2012.

(23) Choi, H. S.; Liu, W.; Misra, P.; Tanaka, E.; Zimmer, J. P.; Ipe, B. I.; Bawendi, M. G.; Frangioni, J. V. Renal Clearance of Nanoparticles. Nature Biotechnology 2007, 25, 1165-1170.

(24) Cai, Q.-Y.; Kim, S. H.; Choi, K. S.; Kim, S. Y.; Byun, S. J.; Kim, K. W.; Park, S. H.; Juhng, S. K.; Yoon, K.-H. Colloidal Gold Nanoparticles as a BloodPool Contrast Agent for X-ray Computed Tomography in Mice. Investigative Radiology 2007, 42, 797-806.

(25) Longmire, M.; Choyke, P. L.; Kobayashi, H. Clearance properties of nanosized particles and molecules as imaging agents: considerations and caveats. Nanomedicine (Lond) 2008, 3, 703-717. 
(26) Lee, N.; Choi, S. H.; Hyeon, T. Nano-Sized CT Contrast Agents. Advanced Materials 2013, 25, 2641-2660.

(27) Rabin, O.; Manuel Perez, J.; Grimm, J.; Wojtkiewicz, G.; Weissleder, R. An X-ray computed tomography imaging agent based on long-circulating bismuth sulphide nanoparticles. Nature Materials 2006, 5, 118-122.

(28) Kinsella, J. M.; Jimenez, R. E.; Karmali, P. P.; Rush, A. M.; Kotamraju, V. R.; Gianneschi, N. C.; Ruoslahti, E.; Stupack, D.; Sailor, M. J. X-Ray Computed Tomography Imaging of Breast Cancer by using Targeted Peptide-Labeled Bismuth Sulfide Nanoparticles. Angewandte Chemie International Edition.

(29) Pan, D.; Roessl, E.; Schlomka, J.; Caruthers, S. D.; Senpan, A.; Scott, M. J.; Allen, J. S.; Zhang, H.; Hu, G.; Gaffney, P. J.; Choi, E. T.; Rasche, V.; Wickline, S. A.; Proksa, R.; Lanza, G. M. Computed Tomography in Color: NanoK-Enhanced Spectral CT Molecular Imaging. Angewandte Chemie 2010, 122, 9829-9833.

(30) Pan, D.; Williams, T. A.; Senpan, A.; Allen, J. S.; Scott, M. J.; Gaffney, P. J.; Wickline, S. A.; Lanza, G. M. Detecting Vascular Biosignatures with a Colloidal, Radio-Opaque Polymeric Nanoparticle. Journal of the American Chemical Society 2009, 131, 15522-15527.

(31) Hainfeld, J. F.; Slatkin, D. N.; Focella, T. M.; Smilowitz, H. M. Gold nanoparticles: a new X-ray contrast agent. British Journal of Radiology 2006, 79, 248-253. 
(32) Ahn, S.; Jung, S.; Lee, S. Gold Nanoparticle Contrast Agents in Advanced X-ray Imaging Technologies. Molecules 2013, 18, 5858-5890.

(33) Liu, Z.; Li, Z.; Liu, J.; Gu, S.; Yuan, Q.; Ren, J.; Qu, X. Long-circulating Er3+-doped Yb2O3 up-conversion nanoparticle as an in vivo X-Ray CT imaging contrast agent. Biomaterials 2012, 33, 6748-6757.

(34) Torres, A. S.; Bonitatibus, P. J.; Colborn, R. E.; Goddard, G. D.; FitzGerald, P. F.; Lee, B. D.; Marino, M. E. Biological Performance of a SizeFractionated Core-Shell Tantalum Oxide Nanoparticle X-Ray Contrast Agent. Investigative Radiology 2012, 47, 578-587.

(35) Bonitatibus, P. J.; Torres, A. S.; Kandapallil, B.; Lee, B. D.; Goddard, G. D.; Colborn, R. E.; Marino, M. E. Preclinical Assessment of a Zwitterionic Tantalum Oxide Nanoparticle X-ray Contrast Agent. ACS Nano 2012, 6, $6650-6658$.

(36) Kim, D.; Park, S.; Lee, J. H.; Jeong, Y. Y.; Jon, S. Antibiofouling PolymerCoated Gold Nanoparticles as a Contrast Agent for in Vivo X-ray Computed Tomography Imaging. Journal of the American Chemical Society 2007, 129, 7661-7665.

(37) Popovtzer, R.; Agrawal, A.; Kotov, N. A.; Popovtzer, A.; Balter, J.; Carey, T. E.; Kopelman, R. Targeted Gold Nanoparticles enable Molecular CT Imaging of Cancer. Nano Lett 2008, 8, 4593-4596. 
(38) Liu, Y.; Ai, K.; Lu, L. Nanoparticulate X-ray Computed Tomography Contrast Agents: From Design Validation to in Vivo Applications. Accounts of Chemical Research 2012.

(39) Khlebtsov, N.; Dykman, L. Biodistribution and toxicity of engineered gold nanoparticles: a review of in vitro and in vivo studies. Chemical Society Reviews 2011, 40, 1647-1671.

(40) Lasagna-Reeves, C.; Gonzalez-Romero, D.; Barria, M. A.; Olmedo, I.; Clos, A.; Sadagopa Ramanujam, V. M.; Urayama, A.; Vergara, L.; Kogan, M. J.; Soto, C. Bioaccumulation and toxicity of gold nanoparticles after repeated administration in mice. Biochemical and Biophysical Research Communications 2010, 393, 649-655.

(41) De Marcillac, P.; Coron, N.; Dambier, G.; Leblanc, J.; Moalic, J.-P. Experimental detection of [alpha]-particles from the radioactive decay of natural bismuth. Nature 2003, 422, 876-878.

(42) Bismuth metal prices, Bismuth metal ingot prices, Bismuth charts, Bismuth historical prices, Business graphs. http://www.metalprices.com/FreeSite/metals/bi/bi.asp (accessed September 7, 2011).

(43) Briand, G. G.; Burford, N. Bismuth compounds and preparations with biological or medicinal relevance. Chemical Review 1999, 99, 2601-2658.

(44) Bierer, D. W. Bismuth subsalicylate: history, chemistry, and safety. Review of Infections Diseases 1990, 12 Suppl 1, S3-8. 
(45) Lee, S. P. The mode of action of colloidal bismuth subcitrate. Scandinavian Journal of Gastroenterology 1991, 185, 1-6.

(46) Lambert, J. R.; Midolo, P. The actions of bismuth in the treatment of Helicobacter pylori infection. Alimentary Pharmacology \& Therapeutics 1997, 11, 27-33.

(47) Lambert, J. R. Pharmacology of Bismuth-Containing Compounds. Clinical Infectious Diseases 1991, 13, S691-S695.

(48) Serfontein, W. J.; Mekel, R. Bismuth toxicity in man II. Review of bismuth blood and urine levels in patients after administration of therapeutic bismuth formulations in relation to the problem of bismuth toxicity in man. Research Communications Chemical Pathology and Pharmacology 1979, $26,391-411$.

(49) Sadler, P. J.; Li, H.; Sun, H. Coordination chemistry of metals in medicine: target sites for bismuth. Coordination Chemistry Reviews 1999, 185-186, 689-709.

(50) A. Dahlgren, C. Gløgård, M. Gammels Organobismuth Compounds: Activity against Helicobacter pylori. Scandinavian Journal of Gastroenterology 1999, 34, 135-137.

(51) Stratton, C. W.; Warner, R. R.; Coudron, P. E.; Lilly, N. A. Bismuthmediated disruption of the glycocalyx- cell wall of Helicobacter pylori:ultrastructural evidence for a mechanism of action for bismuth salts. Journal of Antimicrobical Chemotherapy 1999, 43, 659-666. 
(52) Wagner, S.; Beil, W.; Mai, U.E.H.; Bokemeyer, C.; Meyer, H.J.; Manns, M.P. Interaction between Helicobacter pylori and human gastric epithelial cells in culture: effect of antiulcer drugs. Pharmacology 1994, 49, 226-237.

(53) Vanhoe, H.; Versieck, J.; Vanballenberghe, L.; Dams, R. Bismuth in human serum: reference interval and concentrations after intake of a therapeutic dose of colloidal bismuth subcitrateClinical Chimica Acta 1993, 219, 79-91.

(54) Nwokolo, C. U.; Gavey, C. J.; Smith, J. T. L.; Pounder, R. E. The absorption of bismuth from oral doses of tripotassium dicitrato bismuthate. Alimentary Pharmacology \& Therapeutics 1989, 3, 29-39.

(55) Hudson, M.; Mowat, N. A. Reversible toxicity in poisoning with colloidal bismuth subcitrate. BMJ: British Medical Journal 1989, 299, 159.

(56) Bradley, B.; Singleton, M.; Po, A. L. W. Bismuth Toxicity-a Reassessment*. Journal of Clinical Pharmacy and Therapeutics 1989, 14, $423-441$.

(57) Sano, Y.; Satoh, H.; Chiba, M.; Okamoto, M.; Serizawa, K.; Nakashima, H.; Omae, K. Journal of Occupational Health 2005, 47, 293-298.

(58) Zhao, Y.; Zhang, Z.; Dang, H. A simple way to prepare bismuth nanoparticles. Materials Letters 2004, 58, 790-793.

(59) Wang, Y.; Xia, Y. Bottom-Up and Top-Down Approaches to the Synthesis of Monodispersed Spherical Colloids of Low Melting-Point Metals. Nano Letters 2004, 4, 2047-2050. 
(60) Wang, F.; Tang, R.; Yu, H.; Gibbons, P. C.; Buhro, W. E. Size- and ShapeControlled Synthesis of Bismuth Nanoparticles. Chemistry of Materials 2008, 20, 3656-3662.

(61) Yarema, M.; Kovalenko, M. V.; Hesser, G.; Talapin, D. V.; Heiss, W. Highly Monodisperse Bismuth Nanoparticles and Their Three-Dimensional Superlattices. Journal of the American Chemical Society 2010, 132, $15158-15159$.

(62) Richards, V. N.; Shields, S. P.; Buhro, W. E. Nucleation Control in the Aggregative Growth of Bismuth Nanocrystals. Chemistry of Materials 2011, $23,137-144$.

(63) Wang, F.; Buhro, W. E. An easy shortcut synthesis of size-controlled bismuth nanoparticles and their use in the SLS growth of high-quality colloidal cadmium selenide quantum wires. Small 2010, 6, 573-581.

(64) Schulz, S.; Heimann, S.; Wölper, C.; Assenmacher, W. Synthesis of Bismuth Pseudocubes by Thermal Decomposition of Bi2Et4. Chemistry of Materials 2012, 24, 2032-2039.

(65) Warren, S. C.; Jackson, A. C.; Cater-Cyker, Z. D.; DiSalvo, F. J.; Wiesner, U. Nanoparticle Synthesis via the Photochemical Polythiol Process. Journal of the American Chemical Society 2007, 129, 10072-10073.

(66) Wang, Y.; Chen, J.; Chen, L.; Chen, Y.-B.; Wu, L.-M. Shape-Controlled Solventless Syntheses of Nano Bi Disks and Spheres. Crystal Growth \& Design 2010, 10, 1578-1584. 
(67) Son, J. S.; Park, K.; Han, M.; Kang, C.; Park, S.; Kim, J.; Kim, W.; Kim, S.; Hyeon, T. Large-Scale Synthesis and Characterization of the Size-Dependent Thermoelectric Properties of Uniformly Sized Bismuth Nanocrystals. Angewandte Chemie International Edition 2011, 50, 13631366.

(68) Wang, Y. W.; Hong, B. H.; Kim, K. S. Size Control of Semimetal Bismuth Nanoparticles and the UV-Visible and IR Absorption Spectra. The Journal of Physical Chemistry B 2005, 109, 7067-7072.

(69) Hossain, M.; Luo, Y.; Sun, Z.; Wang, C.; Zhang, M.; Fu, H.; Qiao, Y.; Su, M. X-ray enabled detection and eradication of circulating tumor cells with nanoparticles. Biosensors and Bioelectronics 2012, 38, 348-354.

(70) Luo, Y.; Wang, C.; Qiao, Y.; Hossain, M.; Ma, L.; Su, M. In vitro cytotoxicity of surface modified bismuth nanoparticles. Journal of Materials Science: Materials in Medicine 1-11.

(71) Fievet, F.; Lagier, J.; Blin, B.; Beaudoin, B.; Figlarz, M. Homogeneous and heterogeneous nucleations in the polyol process for the preparation of micron and submicron size metal particles. Solid State lonics 1989, 32-33, 198-205.

(72) Goia, C.; Matijević, E.; Goia, D. V. Preparation of Colloidal Bismuth Particles in Polyols. Journal of Materials Research 2011, 20, 1507-1514. 
(73) Wang, Y.; Kim, K. S. Large-scale polyol synthesis of single-crystal bismuth nanowires and the role of $\mathrm{NaOH}$ in the synthesis process. Nanotechnology 2008, 19, 265303.

(74) Wang, J.; Wang, X.; Peng, Q.; Li, Y. Synthesis and Characterization of Bismuth Single-Crystalline Nanowires and Nanospheres. Inorganic Chemistry 2004, 43, 7552-7556.

(75) Li, J.; Fan, H.; Chen, J.; Liu, L. Synthesis and characterization of poly(vinyl pyrrolidone)-capped bismuth nanospheres. Colloids and Surfaces A: Physicochemical and Engineering Aspects 2009, 340, 66-69.

(76) Zou, C. D.; Gao, Y. L.; Yang, B.; Zhai, Q. J. Melting and undercooling of bismuth nanocrystals by solvothermal synthesis. Physica B: Condensed Matter 2009, 404, 4045-4050.

(77) Wang, W. Z.; Poudel, B.; Ma, Y.; Ren, Z. F. Shape Control of Single Crystalline Bismuth Nanostructures. The Journal of Physical Chemistry B 2006, 110, 25702-25706.

(78) Cheng, G.; Wu, J.; Xiao, F.; Yu, H.; Lu, Z.; Yu, X.; Chen, R. Synthesis of bismuth micro- and nanospheres by a simple refluxing method. Materials Letters 2009, 63, 2239-2242.

(79) Chen, Y.; Gong, R.; Zhang, W.; Xu, X.; Fan, Y.; Liu, W. Synthesis of singlecrystalline bismuth nanobelts and nanosheets. Materials Letters 2005, 59, $909-911$. 
(80) Foos, E. E. Synthesis of Nanocrystalline Bismuth in Reverse Micelles. Journal of the American Chemical Society 122, 7114-7115.

(81) Fang, J.; Stokes, K. L.; Zhou, W. L.; Wiemann, J. A.; Dai, J.; Oconnor, C. J. COLLOIDAL BISMUTH NANOPARTICLES: SYNTHESIS AND UV-VIS ABSORPTION. World Scientific Publishing Co. Pte. Ltd., 2000; pp. 91-96.

(82) Fang, J.; Stokes, K. L.; Wiemann, J. A.; Zhou, W. L.; Dai, J.; Chen, F.; O'Connor, C. J. Microemulsion-processed bismuth nanoparticles. Materials Science and Engineering B 2001, 83, 254-257.

(83) Gutierrez, M.; Henglein, A. Nanometer-Sized Bi Particles in Aqueous Solution: Absorption Spectrum and Some Chemical Properties. Journal of Physical Chemistry 1996 100(18), 7656-7661

(84) Wang, Y.; Zhao, J.; Zhao, X.; Tang, L.; Li, Y.; Wang, Z. A facile waterbased process for preparation of stabilized Bi nanoparticles. Materials Research Bulletin 2009, 44, 220-223.

(85) Ma, D.; Zhao, J.; Zhao, Y.; Hao, X.; Li, L.; Zhang, L.; Lu, Y.; Yu, C. Synthesis of bismuth nanoparticles and self-assembled nanobelts by a simple aqueous route in basic solution. Colloids and Surfaces $A$ : Physicochemical and Engineering Aspects 2012, 395, 276-283.

(86) Fang, J.; Stokes, K. L.; Wiemann, J.; Zhou, W. Nanocrystalline bismuth synthesized via an in situ polymerization-microemulsion process. Materials Letters 2000, 42, 113-120. 


\section{Chapter 2 - pH-dependent Synthesis and Stability of Aqueous, Elemental Bismuth Glyconanoparticle Colloids: Potentially Biocompatible X-ray Contrast Agents}

Reproduced with permission from:

Anna L. Brown and Andrea M. Goforth, Chemistry of Materials 201224 (9), 1599-1605.

Copyright 2012 American Chemical Society.

\subsection{Abstract}

Taking advantage of a $\mathrm{pH}$-dependent solubility equilibrium, we have developed an aqueous synthesis of chemically and colloidally stable bismuth(0) glyconanoparticles. The synthetic method results in potentially biocompatible elemental Bi nanoparticles (BiNPs) and involves the reduction of aqueous $\mathrm{Bi}(\mathrm{III})$ cations by sodium borohydride in a $\mathrm{pH}$-controlled solution. Medical grade dextran (75,000 MW) was found to protect the nanocrystals from oxidation, in addition to promoting colloidal stability and separation of individual nanocrystallites. The rate of particle formation was dependent on synthesis $\mathrm{pH}$, and decreasing the reaction rate by increasing the $\mathrm{pH}$ produced a greater number of individual and isolated $\mathrm{Bi}(0)$ nanocrystals. Stable, aqueous colloids of the dextran-coated BiNPs decomposed under prolonged light exposure, and the NPs dissolved both in acidic solutions $(\mathrm{pH}<7)$ and highly alkaline solutions $(\mathrm{pH}>12)$, but were stable in 
phosphate buffered saline solution $(\mathrm{pH}=7.4)$ and in other aqueous solutions between $\mathrm{pH} 8$ and 10. Bismuth based nanomaterials have previously been demonstrated to be long circulating X-ray contrast agents, and we anticipate that these bismuth(0) glyconanoparticles will find use in similar applications.

\subsection{Introduction}

Bismuth nanoparticles (BiNPs) have garnered attention recently due to their predicted quantum size effects, $(1,2)$ favorable thermoelectric properties, $(3,4,5)$ and use as seeds for VLS growth of metal nanowires. $(6,7)$ Additionally, the potential of Bi-based nanomaterials has very recently been explored as high contrast, long circulating, low-toxicity X-ray contrast agents (XCAs). $(8,9,10)$ Nanometer-sized inorganic particles are attractive as XCAs since they contain a large number (hundreds or more) of X-ray attenuating atoms in a relatively small volume, which should allow for intravenous administration at low concentrations, particularly if the particles are site-directed by the addition of biological recognition moieties to the surface.(11) The inherent high surface area to volume ratio characteristic of nanoparticles can be further advantageous in allowing for the addition of high copy numbers of recognition groups to the NP surface, thus taking advantage of recognized multi-valency effects in certain nanoparticle-biological structure honing interactions.(12)

Bismuth is an attractive element for nanomaterial XCAs due to its high atomic number $(Z=83)$ and well-known biological tolerance.(13) The first of 
these properties will make bismuth-based contrast agents more X-ray opaque on a per atom basis than current, clinically used, iodine-based $(Z=53)$ contrast agents; the second should allow for safe use during imaging as well as biological clearance after imaging. As an alternative to bismuth-based nanoparticles, heavy element gold nanoparticles (AuNPs, $Z=79$ ) have begun to be explored as nano XCAs, $(14,15,16)$ primarily due to the ease of synthesis and morphological control that has been demonstrated for AuNPs over the last several decades. However, gold is currently about two thousand times more expensive per mole(17) and approximately half as terrestrially abundant as bismuth,(18) making AuNPs substantially less attractive than BiNPs for large-scale, systematic medical use.(19) Most importantly, while the oxidative inertness of AuNPs confers a synthetic advantage in the laboratory, AuNPs larger than $5 \mathrm{~nm}$ pose a toxicity risk since they would not be renally cleared,(11) thus making bio-accumulation likely.(20) Conversely, elemental BiNPs readily undergo oxidation and hydrolysis in water, rendering them difficult to synthesize and stabilize in an aqueous environment while simultaneously making them more likely to decompose to small, renally clearable, molecular or ionic $\mathrm{Bi}(\mathrm{III})$ species in vivo. Medically, $\mathrm{Bi}(\mathrm{III})$ chelate solutions have been used for centuries in relatively high doses (multiple gram doses per day)(21) as safe anti-microbial and anti-emetic treatments with minimal known toxicity, despite the fact that these preparations have been predominantly chemically ill-defined.(13) $\mathrm{Bi}$ (III) complexes present in blood are thought to be cleared by urinary excretion via metallothionine, a cysteine rich 
protein abundant in the kidneys that has been shown to have preferential affinity for bismuth over other elements, regardless of $\mathrm{pH} .(22)$

While the hydrolytic instability of BiNPs may prove advantageous for a medical application by providing a mechanism (i.e., biocorrosion) for clearance, aqueous synthesis of BiNPs is a substantial challenge as is the stabilization of aqueous BiNPs colloids for a time period suitable as an imaging window (up to 24 hours). The majority of BiNP preparations reported to date are carried out anaerobically using organic solvents and morphology controlling surfactants of poor or unknown biocompatibility. These syntheses primarily yield hydrophobic BiNPs or present challenges for purification from excess, potentially toxic reagents prior to a biological application. The elegant synthetic preparations of BiNPs by Buhro and others from $\mathrm{Bi}\left(\mathrm{N}\left(\mathrm{SiMe}_{3}\right)_{2}\right)_{3}$ produce a variety of wellcontrolled shapes and sizes. $(23,24,25)$ However the use of hydrophobic solvents and a specialty block co-polymer for stability makes the products of this preparation method non-ideal for medical application. Variations of polyol preparations have been reported for the synthesis of $\mathrm{Bi}$ nano-structures in ethylene glycol, including spheres,(26,27) cubes,(28) and wires.(29) In these preparations, PVP is used as a surfactant, stabilizer and morphology-directing agent. Although the use of PVP may confer some degree of aqueous stability to the particles after work-up, we were unable to find literature evidence that PVP prevents surface oxidation and eventual hydrolysis of elemental BiNPs, which is commensurate with our laboratory experience with this capping agent. A unique, 
aqueous preparation of BiNPs uses oleate as a surfactant to form a stabilizing barrier against BiNP hydrolysis;(30) however, this hydrophobic barrier also prevents colloidal dispersion of the nanocrystals in water. To date, an aqueous synthesis method employing biocompatible reagents that yields small $(<200 \mathrm{~nm}$ hydrodynamic diameter), colloidally stable, hydrophilic BiNPs has not been reported. An aqueous synthetic preparation (which reduces purification steps and minimizes toxicity risks), hydrophilicity, and intermediate hydrolytic stability are all requisites for a suitable nanomaterial XCA.

Herein, we describe a solution method for the aqueous synthesis and aqueous stabilization of elemental BiNPs, which are produced by a simple, benchtop $\mathrm{Bi}(\mathrm{III})$ salt reduction using biocompatible reagents. We also examined the quality (size, morphology, extent of aggregation, and period of colloidal/chemical stability) of the BiNP products to determine synthesis and storage conditions suitable for future utilization of these high atomic number NPs in medical imaging. We used $\mathrm{Bi}\left(\mathrm{NO}_{3}\right)_{3} \cdot 5 \mathrm{H}_{2} \mathrm{O}$ treated with varying amounts of $\mathrm{KOH}$ and a standard amount of glycine to control $\mathrm{pH}$, and thus the concentration of $\mathrm{Bi}(\mathrm{III})$ monomer available for reduction. Short chain medical grade dextran (75,000 MW) was used as a biocompatible surfactant in the preparation, and sodium borohydride was used as the reducing agent. Dextran is a glucose polymer used medically as a plasma volume enhancer and is also commonly used to solubilize iron as an intravenous treatment for anemia. In this preparation, dextran serves to surface stabilize the BiNPs while promoting 
colloidal stability in aqueous media and providing reasonably long-term protection against hydrolysis, both of which (colloidal and chemical stability) are observed to be dependent on $\mathrm{pH}$. While the effect of $\mathrm{OH}^{-} / \mathrm{Bi}(\mathrm{III})$ ratio on the morphology of $\mathrm{Bi}$ nanostructures has been reported in non-aqueous media (i.e., ethylene glycol)(29) and the effect of $\mathrm{OH}^{-} / \mathrm{Bi}(\mathrm{III})$ ratio on the kinetics of aqueous bismuth hydroxide $\left(\mathrm{Bi}(\mathrm{OH})_{3}\right)$ particle formation has been examined,(31) to our knowledge, this work constitutes the first report on the effect of $\mathrm{pH}$ in controlling the aqueous synthesis and stability of elemental BiNP.

\subsection{Experimental}

\section{Reagents}

Bismuth nitrate pentahydrate (Acros, $98 \%$ ), glycine (Acros, $99+\%$, analysis grade), $\mathrm{KOH}$ (reagent grade), dextran (Carbomer, Inc., 75,000 MW, clinical grade), sodium borohydride (MP Biomedicals, 98-99\%), and phosphoric acid (Sigma-Aldrich, $>85 \%$ ) were purchased and used as received without further purification. All solutions were prepared using fresh, electrophoretically pure (18 $\mathrm{M} \Omega \cdot \mathrm{cm}$ resistivity) $\mathrm{H}_{2} \mathrm{O}$.

\section{Synthesis}

All reactions were performed with magnetic stirring at $>800 \mathrm{rpm}$. During the reactions, temperature was held constant at $40^{\circ} \mathrm{C}$ by immersion of the reaction vessel (a $100 \mathrm{~mL}$ beaker) in a temperature controlled water bath. In a 
typical synthesis, $250 \mathrm{mg}(0.52 \mathrm{mmol}) \mathrm{Bi}\left(\mathrm{NO}_{3}\right)_{3} \cdot 5 \mathrm{H}_{2} \mathrm{O}$ was suspended by sonication in $25.0 \mathrm{~mL} \mathrm{H}_{2} \mathrm{O}$. A $2.00 \mathrm{M}$ glycine solution $(10.0 \mathrm{~mL}, 20 \mathrm{mmol}$ ) was added to the $\mathrm{Bi}(\mathrm{III})$ solution, along with an appropriate amount of $2.0 \mathrm{M} \mathrm{KOH}$ to achieve a specified $\mathrm{pH}$ (e.g., $12.0 \mathrm{~mL}, 12 \mathrm{mmol}$, for a measured $\mathrm{pH}$ of 9.0 ). Then, $7.5 \mathrm{~mL}$ of a $100 \mathrm{mg} / \mathrm{mL}$ dextran stock solution $(750 \mathrm{mg}$ ) was added immediately prior to the addition of $12.5 \mathrm{~mL}$ of a freshly prepared $10 \mathrm{mg} / \mathrm{mL}(3.3 \mathrm{mmol})$ $\mathrm{NaBH}_{4}$ solution. Upon addition of reducing agent, the solution was observed to change from a cloudy, colorless suspension to a black solution in a time frame that was dependent on the initial $\mathrm{pH}$ of the reaction. After approximately 5 minutes, when the reactions appeared to have stabilized by visual inspection (based on color change) and cessation of gas evolution $\left(\mathrm{H}_{2}\right.$ from $\left.\mathrm{BH}_{4}{ }^{-}\right)$, all solutions were brought to the same $\mathrm{pH}(7.4)$ by the drop wise addition of $1.0 \mathrm{M}$ $\mathrm{H}_{3} \mathrm{PO}_{4}$. Samples were briefly sonicated to promote dispersion and allowed to stir for one hour at room temperature prior to purification.

\section{Dialysis and Purification}

The reaction products containing the nanoparticles were dialyzed against water using Spectrum* Spectra/Por $(50,000 \mathrm{MWCO})$ dialysis tubing to separate the nanoparticles from salts, excess starting reagents, and small molecule byproducts. The purified nanoparticle solutions were then centrifuged at 3.0 RCF for $10 \mathrm{~min}$ to remove large aggregates. Nanoparticles in the supernatant were concentrated by rotary evaporation under vacuum $\left(40^{\circ} \mathrm{C}\right.$ water bath) and stored 
at room temperature away from light. For long-term storage, samples were stored in water either at $-20^{\circ} \mathrm{C}$ or at $4^{\circ} \mathrm{C}$ with a trace amount of sodium azide to prevent biological contamination.

Spectroscopic Analysis of Particle Nucleation, Growth, and Stability as a Function of $\mathrm{pH}$

In order to monitor the reaction progress as a function of $\mathrm{pH}$ spectroscopically, the reaction was scaled down to keep absorbance/scattering values under 2 for the duration of the reactions. A dilute bismuth nitrate stock solution of $2.06 \mathrm{mM}$ dissolved $\mathrm{Bi}\left(\mathrm{NO}_{3}\right)_{3} \cdot 5 \mathrm{H}_{2} \mathrm{O}$ was prepared and sonicated to optical clarity. Glycine, dextran, and $\mathrm{KOH}$ stock solutions were also prepared and were of the same concentrations as used in the larger-scale preparations. Each reaction solution contained a standard final amount of $\mathrm{Bi}(\mathrm{III})(5.15 \mu \mathrm{mol})$, glycine (100 $\mu \mathrm{mol})$, and dextran $(75 \mathrm{mg}) . \mathrm{KOH}$ was added to achieve desired $\mathrm{pH}$ values in the range 8-10. Preparations for the reactions took place in the following order: 1) appropriate volumes of the $\mathrm{Bi}(\mathrm{III})$, glycine, and $\mathrm{KOH}$ solutions were added to and mixed in $8.0 \mathrm{~mL} \mathrm{H} \mathrm{H}_{2} \mathrm{O}$, 2) the $\mathrm{pH}$ was measured, 3) dextran solution was added, and 4) $\mathrm{H}_{2} \mathrm{O}$ was added as needed to bring the final volume of each solution to $9.9 \mathrm{~mL}$. Each sample was then divided into three equal volume aliquots, which were monitored simultaneously by UV-visible spectroscopy on a Varian Cary 100 Bio equipped with stirring capabilities. Light attenuation (due to absorption and scattering by the BiNPs) was measured at $650 \mathrm{~nm}$ every 5 
seconds after the addition of sodium borohydride solution (37.5 $\mu \mathrm{L}, 10.57 \mu \mathrm{mol})$ to each cuvette at $t=0 \mathrm{~s}$. Data were collected simultaneously from replicates at each $\mathrm{pH}$ and recorded in parallel. Attenuation values at each time point from the three replicates for each $\mathrm{pH}$ were averaged and plotted versus elapsed time. For consistency, all samples were prepared, mixed, and measurements initiated within 90 seconds.

Though all reaction conditions were not exactly identical to those used in the large-scale preparations due to measurement requirements, these smallscale spectroscopic experiments allow real-time observation of reaction stages and trends in reaction progress (i.e., rates) as a function of $\mathrm{pH}$; qualitatively, the same trends in nucleation periods and growth rates are observed in the largescale preparations, although the colloids produced in the large-scale preparations are, in general, colloidally stable for longer time periods (days versus hours).

\section{Product Characterization}

UV-visible spectra for the products of the large-scale preparations were collected on a Shimadzu UV-2450 UV-visible spectrophotometer in standard 1cm disposable polystyrene cuvettes.

Dynamic Light Scattering (DLS) measurements were taken on a Horiba LB-550 dynamic light scattering instrument. Samples were purified as described above, diluted in water, and passed through a $0.45 \mu \mathrm{m}$ PTFE syringe filter prior to measurement. Measurements (300 scans) at 5 different concentrations of each 
sample were performed in order to determine a size distribution independent of concentration effects (e.g., multiple scattering).

Transmission Electron Microscopy (TEM) was performed on an FEI Tecnai F-20 TEM operating at 200 kV. Purified samples were drop-cast on Ted Pella holey carbon copper supported grids and dried for 2 hours at $150^{\circ} \mathrm{C}$ prior to imaging. Energy Dispersive X-ray (EDX) spectroscopy was performed on a representative sample of 20 individual particles during TEM imaging.

FT-IR spectroscopy was performed on a Thermo Scientific Nicolet iS10 spectrophotometer equipped with a single-bounce diamond attenuated total reflectance (ATR) attachment. Aqueous solutions of purified BiNPs, dextran, and glycine were drop-cast onto the ATR crystal and the solvent was evaporated as necessary using a heat gun to deposit a sample film for analysis.

For XRD analysis, concentrated BiNP samples were evaporated to dryness in a ceramic evaporation dish in air, milled into a fine powder, and pressed onto a glass slide. Data were collected in focused beam (BraggBrentano) geometry on a Rigaku Ultima IV X-ray diffraction system using graphite monochromatized $\mathrm{Cu} \mathrm{K} \alpha$ radiation. Scans were performed over the angular range $5-85^{\circ} 2 \theta$ at a scan rate of $0.25 \%$ min at room temperature. 


\section{Results and Discussion}

\subsection{Synthesis}

For future X-ray contrast applications, we have developed an aerobic, aqueous solution method for the preparation of chemically and colloidally stable, water-soluble, potentially biologically compatible BiNPs, which should have the following advantages over existing BiNP preparations: 1) it negates the need for

phase transfer steps post-synthesis, 2) it simplifies preparation and purification steps in employing highly biocompatible starting materials, and 3) it reduces overall environmental impact. The starting material, bismuth nitrate pentahydrate, was chosen as an inexpensive, readily available, and easily dissolved (at low pH, or in dilute aqueous solutions) $\mathrm{Bi}(\mathrm{III})$ source. To reduce $\mathrm{Bi}(\mathrm{III})$ to elemental form, sodium borohydride was chosen, as this reducing agent should decompose in aqueous solution to gaseous products ( $\mathrm{H}_{2}$ and borates) and a soluble sodium salt that can easily be removed by dialysis. A surface stabilizing ligand or polymer was necessary to achieve chemically and colloidally stable, elemental BiNP, and in our preparation, the glucose polymer dextran was found to impart both oxidative and colloidal stability to the BiNPs if the particles were formed slowly (i.e., over a duration of $1-10$ minutes). The solubility of the $\mathrm{Bi}(\mathrm{III})$ source as a function of $\mathrm{pH}$ was found to dramatically affect the extent of aggregation and colloidal stability of the BiNP products (Scheme 2.1), which we discuss below. 


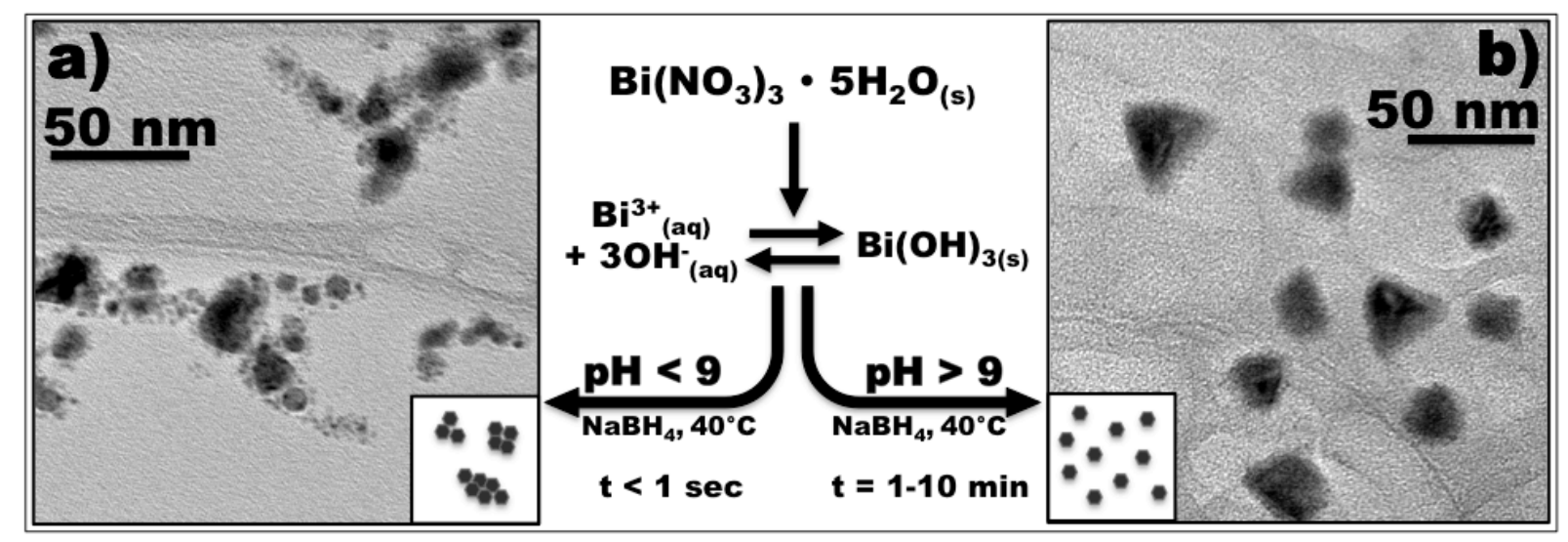

Scheme 2.1. pH-Dependent Synthesis of Colloidally Stable Glyco-BiNPs

Visibly we observed a reaction progression in which both nucleation period and growth rate are dependent on synthesis $\mathrm{pH}$. Treatment of a colorless solution or white suspension of $\mathrm{Bi}\left(\mathrm{NO}_{3}\right)_{3} \cdot 5 \mathrm{H}_{2} \mathrm{O}$ in the presence of glycine and dextran with reducing agent resulted in the evolution of a brown, and ultimately a black, solution color (Figure 2.1 a-e). The elapsed time to obtain an initial brown color, indicative of the beginning of particle nucleation, and the elapsed time to obtain the stable black color, indicative of the formation of larger bismuth(0) nanocrystallites, were noticeably longer with increased reaction $\mathrm{pH}$. In the absence of the carbohydrate surfactant, black BiNP were visibly formed (the rhombohedral bismuth( 0 ) phase was confirmed by PXRD) upon addition of the reducing agent to the aqueous $\mathrm{Bi}(\mathrm{III})$ precursor. However, these bismuth(0) particles quickly aggregated, precipitated out of solution, and eventually underwent oxidation and hydrolysis, resulting in the formation of an unidentified, white amorphous solid. Using glycine as a biocompatible buffering agent with 
amine $\mathrm{pK}_{\mathrm{a}}$ of 9.8 and medical grade $75,000 \mathrm{MW}$ dextran as a polymeric surface stabilizer, we spectroscopically determined the optimal $\mathrm{pH}$ range for the synthesis of colloidally stable, aqueous BiNPs that do not aggregate or decompose to oxidized products (e.g., $\mathrm{Bi}(\mathrm{III})$ solids, such as $\mathrm{Bi}(\mathrm{OH})_{3}$ or $\mathrm{Bi}_{2} \mathrm{O}_{3}$, or $\mathrm{Bi}(\mathrm{III})$ solution species, such as $\mathrm{BiO}_{2}{ }^{-}$or $\mathrm{BiO}_{3}{ }^{3-}$ ) for periods as long as 12 months when appropriately (vide infra) stored in aqueous solution.

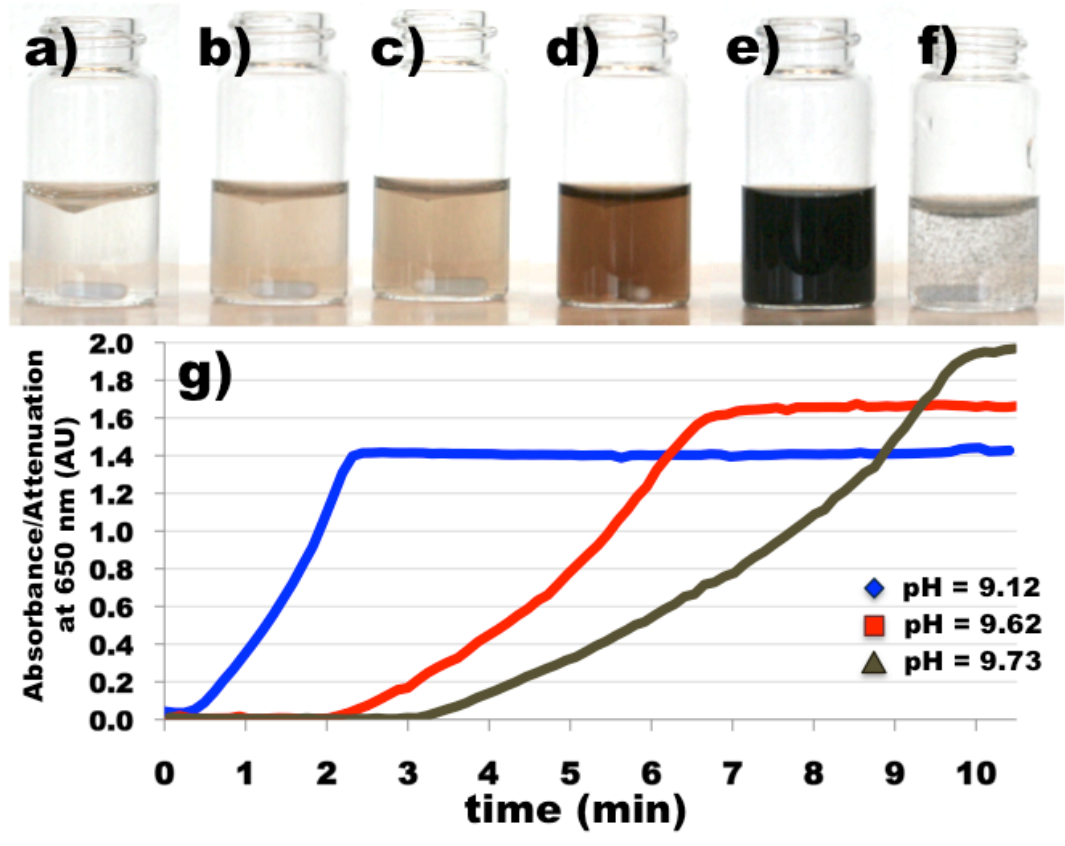

Figure 2.1. Nucleation period and growth rate of BiNPs are dependent on the reaction $\mathrm{pH}$. (a-f) Treating a colorless solution or white suspension of bismuth nitrate in the presence of glycine and dextran (panel a) with reducing agent leads to the development (panels $b-e$ ) of a black colloid of bismuth nanoparticles (e) in a time frame that is $\mathrm{pH}$ - dependent. Particle synthesis in the absence of surfactant or at $\mathrm{pH}<8$ resulted in unstable aggregates (f). (g) Reaction progression monitored by light attenuation at $650 \mathrm{~nm}$ for solutions at measured pH values of 9.12 (blue), 9.62 (red), or 9.73 (green). 
The effect of $\mathrm{pH}$ on the reaction progression and rate is shown in Figure $2.1 \mathrm{~g}$. To monitor the BiNPs formation as a function of $\mathrm{pH}$ in situ, UV-visible spectroscopy was performed on small-scale preparations using initial reagent concentrations that yielded products with suitable attenuation values $(<2$ arbitrary units). Light attenuation, the combination of absorption and scattering, at $650 \mathrm{~nm}$ was measured and is indicative of the reaction progression, i.e., the formation of black, visible light absorbing/scattering particles. The attenuation versus time plots (Fig. 1g) indicate that the reactions proceed in three phases: an initial nucleation phase with no attenuation, a particle growth phase indicated by a dramatic increase in attenuation, and finally a steady-state phase indicated by a plateau in attenuation. Under certain conditions, e.g., low $(<8) \mathrm{pH}$ syntheses, a fourth aggregation phase is observed (Figure $2.1 \mathrm{f}$ ), which is evidenced by loss of attenuation as the particles precipitate (data not shown). The time period of the nucleation phase is strongly $\mathrm{pH}$ dependent and is longer at higher $\mathrm{pH}$ values. The rate of particle growth, as indicated by the change in attenuation with time (i.e., the slope of the growth regime in Fig. 1g), is also $\mathrm{pH}$ dependent and is slower at higher $\mathrm{pH}$ values. Regardless of synthesis $\mathrm{pH}$, all samples reach an absorbance plateau corresponding to a steady state phase in which the composition of the solution does not appear to change with time. Experimentally, we observed that at a low reaction $\mathrm{pH}(\leq 8.0)$, large aggregates of elemental BiNPs were formed in the presence of dextran; these aggregated particles did not oxidize or decompose, as evidenced by the black color of the precipitated 
solid and confirmation of the rhombohedral bismuth(0) phase by PXRD. However, the large aggregates of elemental BiNPs synthesized at $\mathrm{pH} \leq 8.0$ could not be re-dispersed in water, even with durative sonication, to achieve stable aqueous colloids. We found that increasing the reaction $\mathrm{pH}$ (up to $\mathrm{pH}=10.5$ ) produced a greater number of isolated BiNPs (Scheme 2.1) that did not undergo gravitational settling, thus resulting in stable, aqueous Glyco-BiNP colloids. At pH values above 11, no reaction was visually or spectroscopically observed, presumably due to lack of soluble $\mathrm{Bi}(\mathrm{III})$ for reduction (vide infra). For colloidally stable samples synthesized in solutions with $\mathrm{pH}$ values between 9 and 10 , dilute phosphoric acid was added drop wise to achieve final solutions at a physiologically relevant $\mathrm{pH}$ of 7.4 without loss of either colloidal or chemical stability.

A nanoparticle formation mechanism consistent with the $\mathrm{pH}$ effects we observe in the aqueous synthesis is shown in Scheme 2.1. For simplicity we assume that a suspension of solid bismuth hydroxide is in equilibrium with aqueous $\mathrm{Bi}(\mathrm{III})$ and hydroxide ions and that the equilibrium is dependent on $\mathrm{pH}$, with a decreased $\mathrm{Bi}(\mathrm{III})$ concentration achieved by increasing $\mathrm{pH}$ according to LeChatlier's principle. When the soluble $\mathrm{Bi}(\mathrm{III})$ precursor is depleted by reaction with the reducing agent to form bismuth(0) nanoparticles, the solubility equilibrium shifts to replenish the solution precursor at the expense of the precipitate. Thus, the $\mathrm{pH}$-dependent dissolution of the solid should allow for kinetic control over BiNP nucleation and growth by modulating the concentration 
of soluble $\mathrm{Bi}(\mathrm{III})$ monomer available for reduction. Consistent with this, we observe that both nucleation and growth are faster the lower the synthesis $\mathrm{pH}$, which is correspondent with higher available $\mathrm{Bi}(\mathrm{III})$ concentration for reduction in more acidic solutions. Also consistent with this, at $\mathrm{pH} \leq 8$, not enough isolated BiNPs are formed to result in a stable aqueous colloid because the saturation concentration for nucleation and growth is too rapidly achieved, resulting in the formation and precipitation of aggregated nanocrystallites. Notably, the largest difference in the reaction products synthesized at different $\mathrm{pH}$ values is not the individual bismuth nanocrystallite size, but the extent of nanocrystallite aggregation, and thus the size and weight of the glyco-BiNP (where a glyco-BiNP contains one or more than one Bi nanocrystallite). If Bi nanocrystals are formed quickly, as occurs in the lowest $\mathrm{pH}$ reactions, this presents opportunity for multiple nanocrystalline domains to be encapsulated within a single polymeric structure. However, if particles are formed slowly, there are fewer present in solution at any given time prior to particle capping (a fast step) by the polymer, thus reducing the opportunity for inclusion of multiple $\mathrm{Bi}$ nanocrystals in a single polymer structure and, consequently, reducing the Glyco-BiNP size and tendency for gravitational settling. 


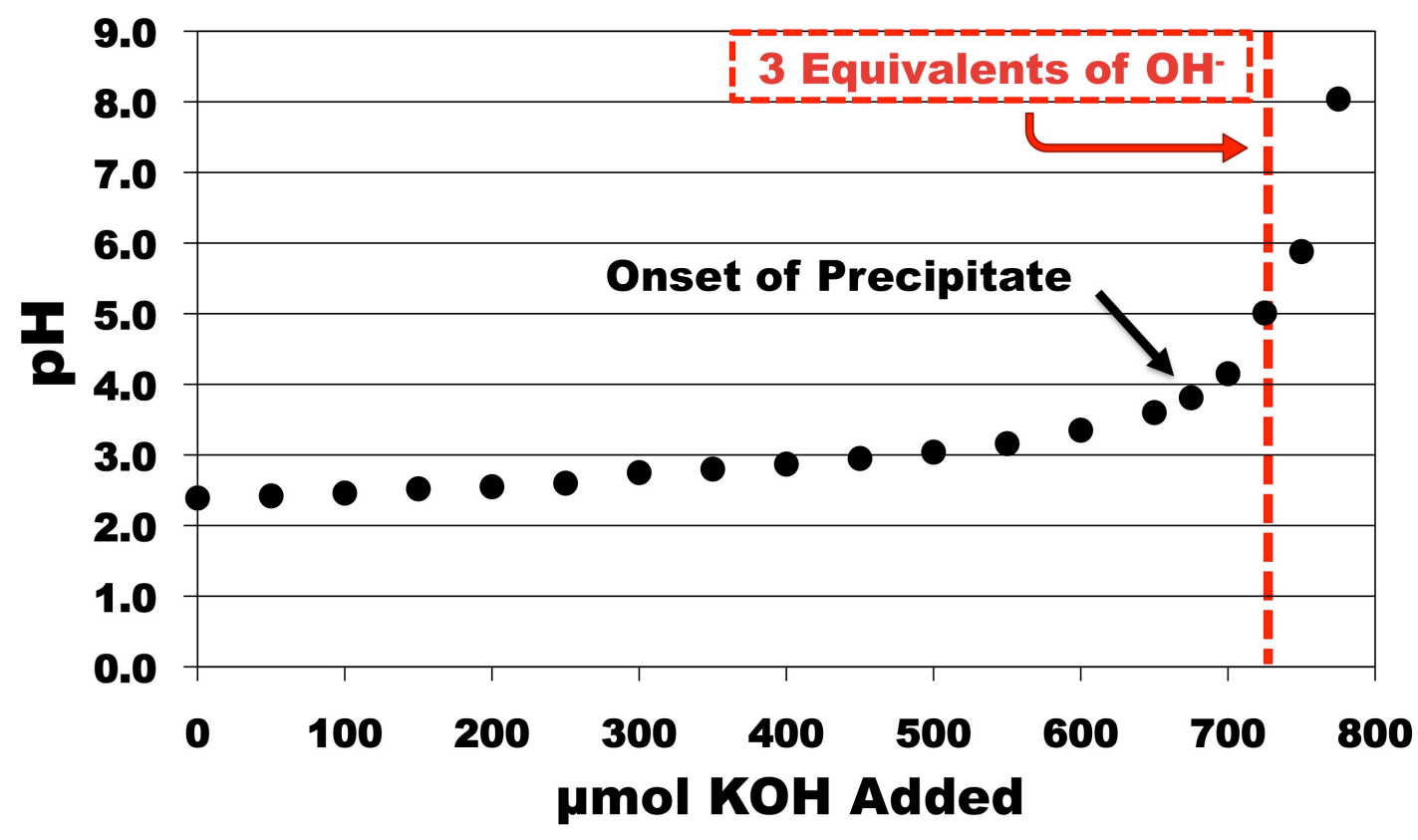

Figure 2.2. Titration of aqueous $\mathrm{Bi}(\mathrm{III})$. $\mathrm{Bi}\left(\mathrm{NO}_{3}\right)_{3} \cdot 5 \mathrm{H}_{2} \mathrm{O}(242 \mu$ mols $)$ was dissolved by sonication in $100 \mathrm{~mL} \mathrm{H}_{2} \mathrm{O}(2.42 \mathrm{mM})$. Titration with $1.0 \mathrm{M} \mathrm{KOH}$ was performed in increments of $50 \mu \mathrm{mol}(50 \mu \mathrm{L})$ to $700 \mu \mathrm{mol}$ of added base, and increments of $25 \mu \mathrm{mol}(25 \mu \mathrm{L})$ up to $775 \mu \mathrm{mol}$, with the presence of a $\mathrm{pH}$ probe and rapid stirring. Arrow indicates visual onset of precipitation and dashed line indicates calculated 3 molar equivalents of hydroxide.

Thus, the pH-dependence of BiNP colloidal stability is influenced by a complex $\mathrm{pH}$-dependent solubility equilibrium involving sparingly soluble $\mathrm{Bi}(\mathrm{III})$ oxide, suboxide, hydroxide, or perhaps, in this case, glycinate precipitates. Dependent upon the counter-anion, a wide range of oxidized precipitates have been reported for dissolved $\mathrm{Bi}(\mathrm{III})$ salts.(32) Though we made no attempts to identify either the precipitate or the solution precursor, $\mathrm{Bi}(\mathrm{III})$ is known to form a 
highly acidic $\left(\mathrm{pK}_{\mathrm{a}}=1.1\right)$ aquo-cation in dilute solution; in concentrated solution or upon titration with base, aqueous $\mathrm{Bi}(\mathrm{III})$ is expected to form an insoluble basic salt that will be in $\mathrm{pH}$-dependent equilibrium with a soluble $\mathrm{Bi}(\mathrm{III})$ species.(31)'(33) Consistent with this, the Pourbaix diagram for bismuth in aerated water indicates that the predominant form of bismuth will be an oxidized solid over most of the $\mathrm{pH}$ range.(34) A titration curve for $\mathrm{Bi}\left(\mathrm{NO}_{3}\right)_{3} \cdot 5 \mathrm{H}_{2} \mathrm{O}$ (Figure 2.2) indicates that the onset of precipitation in dilute solution (2.42 $\mathrm{mM})$ occurs at approximately $\mathrm{pH}=4$ and prior to the addition of 3 molar equivalents of hydroxide. Qualitatively, the same results were observed when $\mathrm{Bi}\left(\mathrm{NO}_{3}\right)_{3} \cdot 5 \mathrm{H}_{2} \mathrm{O}$ was titrated in the presence of glycine; thus we expected and observed a significant amount of solid precipitated precursor in our syntheses at all tested $\mathrm{pH}$ values, and will be discussed in detail in the following section. However, regardless of the identity of the solution precursor and sparingly soluble solid, controlling $\mathrm{pH}$ will control the $\mathrm{Bi}(\mathrm{III})$ concentration available for reduction, consistent with our spectroscopic observations and proposed aqueous formation mechanism. Notably, the successful aqueous synthesis of hydrophobic, oleatecapped BiNP was performed under basic conditions using hydrazine hydrate.(30)

The impact of a variety of other synthetic factors on particle formation kinetics and product colloidal stability was measured spectroscopically, including the addition of salts (e.g., $\mathrm{NaCl}, \mathrm{KNO}_{3}$ ), buffering agents other than glycine (e.g., bicine, tris), and buffer concentration. In all cases, we observed a pH dependent nucleation and growth period with the colloidal stability correlated to synthesis $\mathrm{pH}$ 
more than any other factor and with the most stable colloids achieved in solutions between $\mathrm{pH}$ values of 9 and 10 .

\subsection{Discussion of Aqueous Bi(III) Chemistry}

The chemistry of aqueous $\mathrm{Bi}(\mathrm{III})$ is complicated by multiple precipitate types and counterion influences. The fully hydrated $\mathrm{Bi}(\mathrm{III})$ cation, i.e., $\left[\mathrm{Bi}\left(\mathrm{OH}_{2}\right)_{6}\right]^{3+}$, can lose up to 3 protons to yield the sparingly soluble, neutral species $\mathrm{Bi}(\mathrm{OH})_{3}\left(\mathrm{OH}_{2}\right)_{3}$ (equivalent to $\mathrm{Bi}(\mathrm{OH})_{3}$ ), which can continue to hydrolyze to the thermodynamically stable and insoluble $\mathrm{Bi}_{2} \mathrm{O}_{3}$. However, the aqueous solution form of $\mathrm{Bi}(\mathrm{III})$ is also dictated by the identity of its counterion, for example, bismuth oxychloride and other oxo-chloride complexes are formed when bismuth chloride is hydrolyzed.(18,35) $\mathrm{Bi}(\mathrm{III})$ nitrate solutions at high $\mathrm{pH}$ include several large rather exotic, hydrolyzed bismuth polyanions, (e.g. $\mathrm{Bi}_{4} \mathrm{O}_{7}, \mathrm{Bi}_{2} \mathrm{O}_{5}$ and $\left.\left[\mathrm{Bi}_{6}(\mathrm{OH})_{12}\right]^{6+}\right)(36,32)$ or sparingly soluble sub-oxides (e.g. bismuth subnitrate $\left.\mathrm{BiO}\left(\mathrm{NO}_{3}\right).\right)(31)$ The Pourbaix $(\mathrm{pH} /$ potential predominance) diagram for bismuth reveals that the predominant species of bismuth in aerated water at a neutral to basic $\mathrm{pH}$ is $\mathrm{Bi}_{2} \mathrm{O}_{3}$ solid, and that at lower $\mathrm{pH}$ values, the fully hydrated cation becomes more stable.(34) Thus, in our syntheses, which yielded colloidally stable particles only in solutions between $\mathrm{pH} 8$ and 11 , a significant amount of suspended $\mathrm{Bi}(\mathrm{III})$ solid should be present initially. We observed this experimentally in our titration (Figure 2.2$)$ of a dilute $(240 \mu \mathrm{M})$, optically clear solution of the $\mathrm{Bi}\left(\mathrm{NO}_{3}\right)_{3} \cdot 5 \mathrm{H}_{2} \mathrm{O}$ starting material with $\mathrm{KOH}$, which we used in our 
syntheses in addition to glycine to adjust $\mathrm{pH}$. Even in dilute solution, we observed the onset of precipitation around a measured $\mathrm{pH}$ of 4 and prior to the addition of three molar equivalents $\mathrm{OH}^{-}$. Qualitatively, the same results were observed when $\mathrm{Bi}\left(\mathrm{NO}_{3}\right)_{3} \cdot 5 \mathrm{H}_{2} \mathrm{O}$ was titrated in the presence of glycine. The titration curve is familiar as that of a weak acid with an equivalence point reached around 3 molar equivalences of $\mathrm{KOH}$. However the exact equivalence point is not easily discerned as various oxide precipitates are formed and disrupted during solution equilibrium. The identity of the $\mathrm{Bi}(\mathrm{III})$ cation and the sparingly soluble solid in equilibrium with it will depend on available countercations (e.g., $\mathrm{Bi}\left(\mathrm{NO}_{3}\right)_{3} \cdot 5 \mathrm{H}_{2} \mathrm{O}$ is known to decompose in $\mathrm{H}_{2} \mathrm{O}$ to a wide variety of oxide complexes).(32) We assume that in our reactions the $\mathrm{Bi}(\mathrm{III})$ oxide precipitate is in equilibrium with its component ions $\left(\mathrm{Bi}^{3+}\right.$ and $\left.3 \mathrm{OH}^{-}\right)$which has be previously studied as a function of hydroxide concentration.(33) Regardless of the identity of the solution precursor and sparingly soluble solid, controlling $\mathrm{pH}$ will control the $\left[\mathrm{Bi}^{3+}\right]$ available for reduction, consistent with our spectroscopic observations and proposed aqueous formation mechanism. 

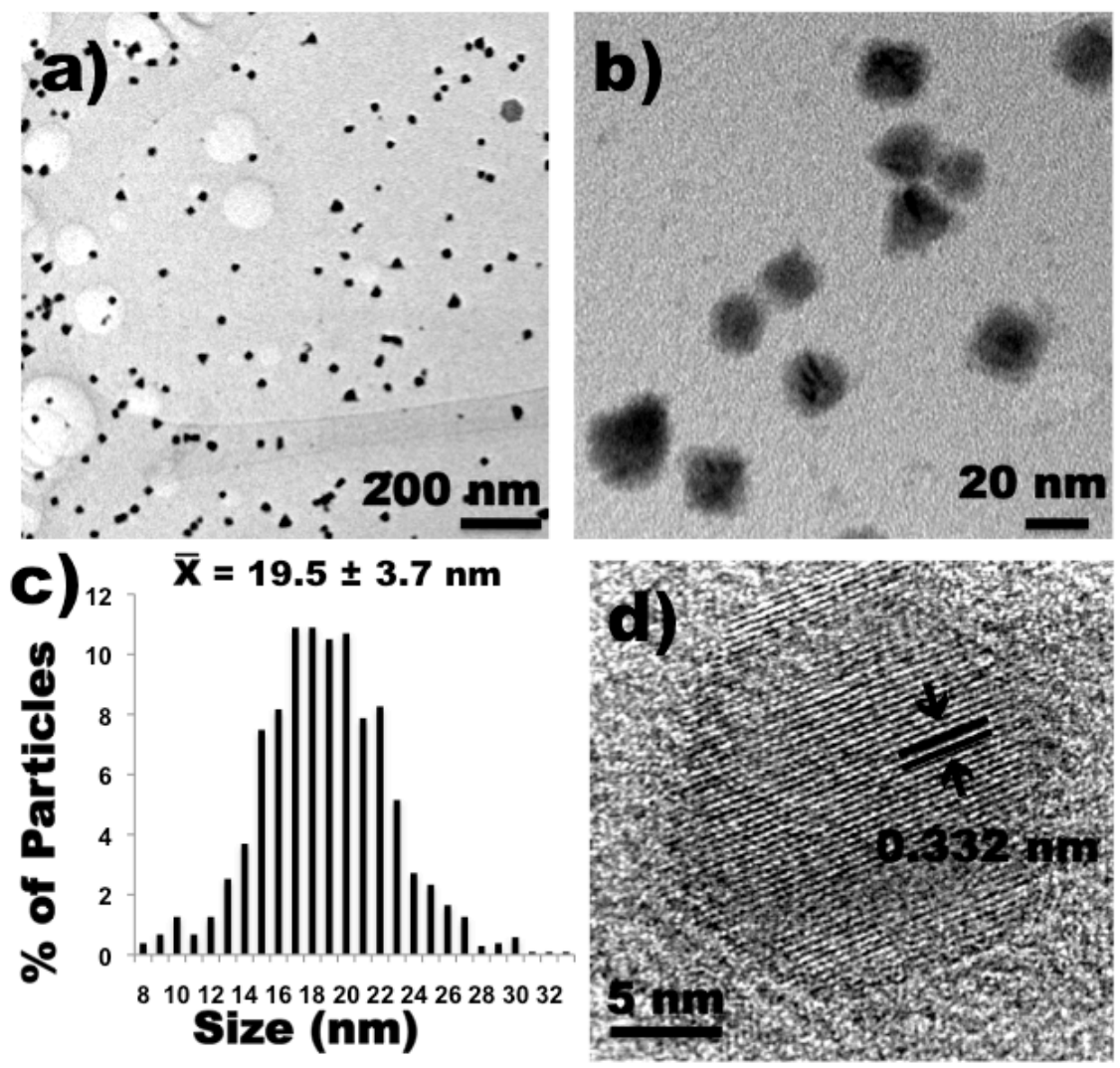

Figure 2.3. ( $a, b, d)$ Transmission Electron Microscopy (TEM) images of BiNPs synthesized at $\mathrm{pH}$ 9.97: $(\mathrm{a}, \mathrm{b})$ the majority of particles appear spherical, although triangles, cubes, and hexagons are also visible. (c) Histogram showing 1040 particles manually measure with a core mean diameter of $19.5 \pm 3.7 \mathrm{~nm}$. (d) High-resolution TEM image of a single particle with line spacing (3.32 Å) characteristic of elemental bismuth.

\subsection{Characterization}

Transmission Electron Microscopy (TEM) was used to analyze BiNP morphology, size, and crystallinity (Figure 2.3 a,b). Various crystallite morphologies were observed, regardless of synthesis $\mathrm{pH}$, including: spheres, triangles, hexagons and rods. However, $>50 \%$ of particles were spherical in all 
cases. TEM images of colloids synthesized at a $\mathrm{pH}$ of 9.97 indicate a freestanding particle diameter of $19.5 \pm 3.7 \mathrm{~nm}$ (Figure $2.3 \mathrm{c}$ ). Under high resolution TEM, a lattice spacing of $0.332 \mathrm{~nm}$ was measured (Figure $2.3 \mathrm{~d}$ ), consistent with the d-spacing $(0.328 \mathrm{~nm})$ corresponding to the [012] planes of bulk elemental bismuth (JCPDS Card No. 00-044-1246). EDX analysis was performed on 20 isolated particles using the same sample grid and only the elements $\mathrm{Bi}, \mathrm{O}, \mathrm{C}$ and $\mathrm{Cu}$ (from the grid support) were found. The presence of bismuth was localized to the particles, i.e., no bismuth was detected outside of the dark crystallites visible on the TEM grid.

The dried colloids were analyzed by powder X-ray diffraction, and the resulting diffraction pattern is largely that of rhombohedral elemental bismuth (Figure 2.4a, blue trace, JCDPS Card No. 00-044-1246, tick marks indicate calculated reflections), consistent with the high resolution TEM analysis. Other reflections apparent in the pattern $\left(17.52,18.83,20.25\right.$, and $\left.28.80^{\circ} 2 \Theta\right)$ were attributed to crystallized dextran (Figure 2.4 a, purple trace).

The FT-IR spectrum (Figure $2.4 \mathrm{~b}$ ) of the dialyzed particles (blue trace) matched the spectrum collected for neat dextran (purple trace). Importantly the absence of carboxylate peaks at 1593 and $1408 \mathrm{~cm}^{-1}$ and amine deformation peaks at 1527 and $1502 \mathrm{~cm}^{-1}$ (37) indicates that glycine served only as a buffering reagent during synthesis and did not become part of the BiNP complex. This was somewhat surprising given that oleic acid and other carboxylate containing species are commonly observed to be surface terminating ligands in 
other BiNP preparations. $(30,38)$ Particles appear to be coated solely in dextran and thus can be accurately termed glyco-BiNP.

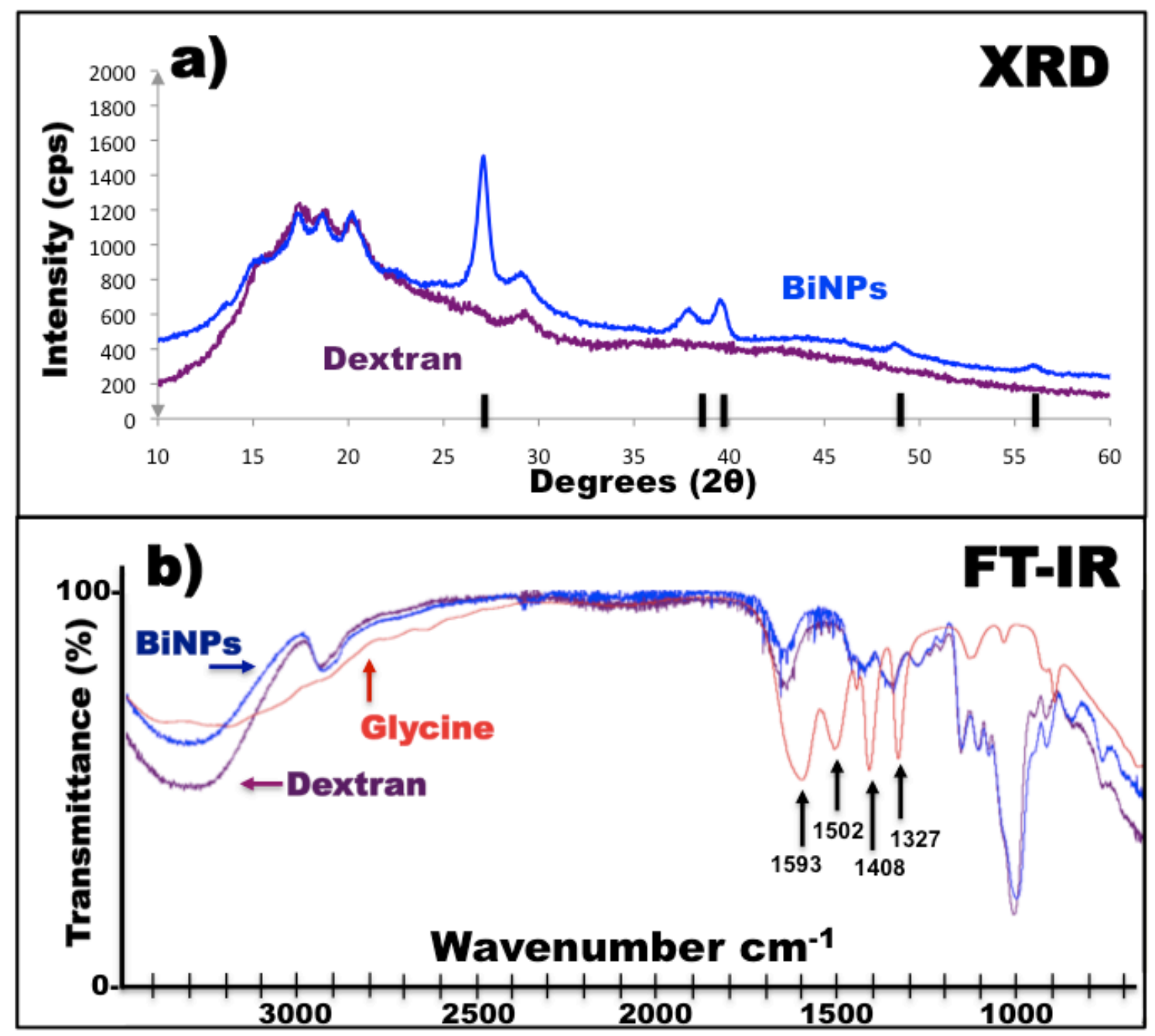

Figure 2.4. X-ray diffraction and FT-IR absorbance of BiNPs. (a) Powder X-ray Diffraction (PXRD) shows BiNPs (blue trace) to be composed of crystallized dextran (purple trace) and elemental bismuth (calculated Bragg reflections for rhombohedral Bi indicated by tick marks). (b) FT-IR spectra of purifed, dextran- stabilized BiNPs thin flms (blue trace), glycine (red trace), and dextran (purple trace). 
Dynamic light scattering (DLS) measurements on colloids synthesized at $\mathrm{pH} 9.97$ indicated the mean hydrodynamic diameter to be $60 \mathrm{~nm}$ after filtration through a $0.45 \mu \mathrm{m}$ syringe filter. The difference $(\sim 40 \mathrm{~nm})$ between the crystallite size $($ diameter $=19.5 \mathrm{~nm})$ measured by TEM and hydrodynamic size $(60 \mathrm{~nm})$ measured by DLS can be attributed to a surfactant and water shell, which presumably imparts colloidal stability and solubility to the particles and prevents or slows hydrolysis and oxidation. DLS data in comparison with TEM data are shown in Figure 2.5. Notably, the syringe filter pre size doesn't appear to affect the crystallite diameter, as measured by TEM, but does decrease the hydrodynamic size, as measured by DLS. This could indicate that either the filter is size selecting a population with a smaller hydrodynamic size, or that the filter is removing some of the organic surfactant on the particles, thus reducing the size of the BiNPs. 


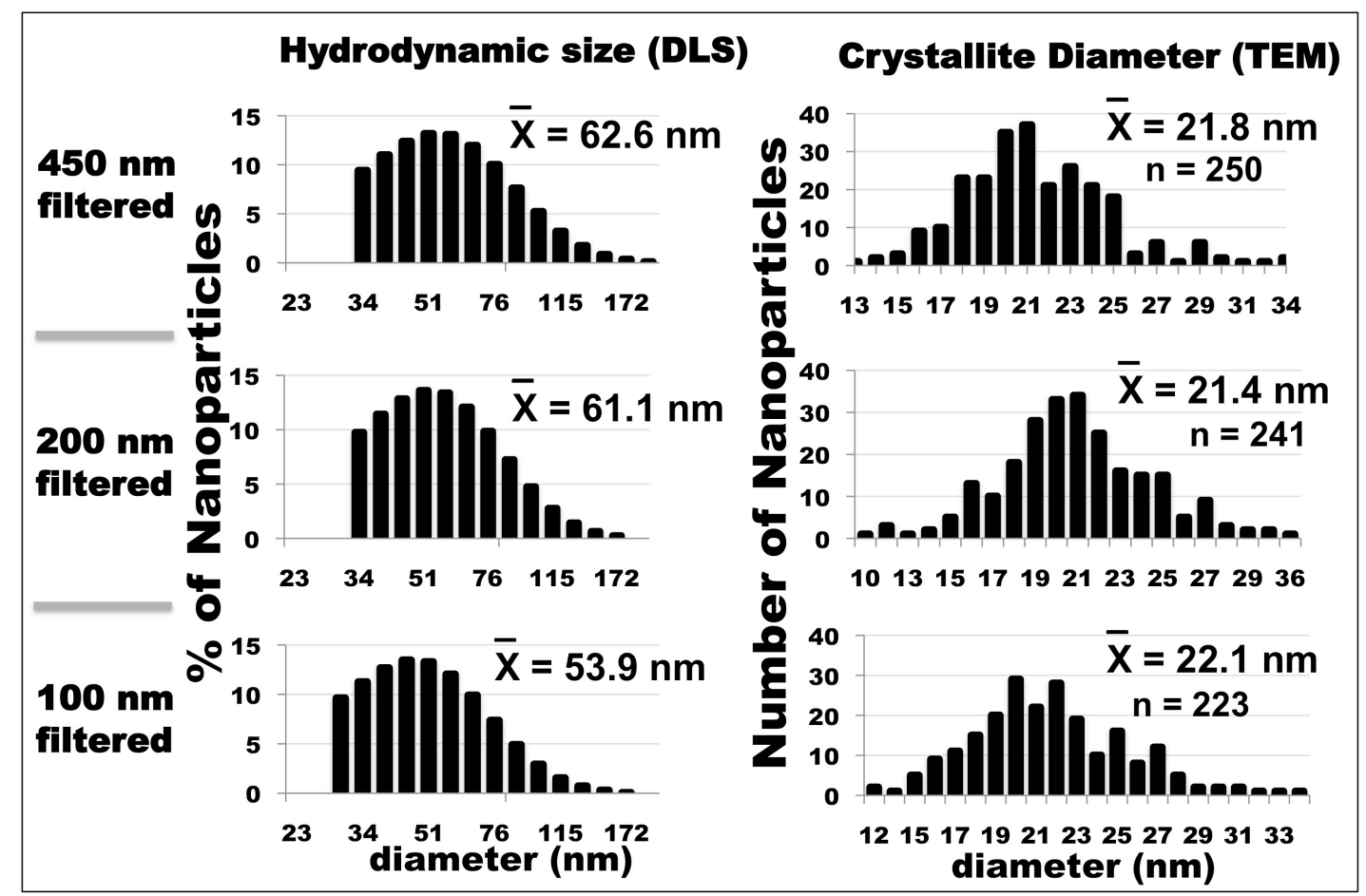

Figure 2.5. Hydrodynamic diameter of BiNP populations change depending on size of syringe filter. Glyco-BiNPs were syringe-filtered through 450,200 , or $100 \mathrm{~nm}$ pore diameter PTFE syringe filters and either diluted in water for DLS analysis or drop cast and dried on a carbon coated TEM grid.

\subsection{Stability and Degradation}

To examine the colloidal stability of the BiNPs over time, purified, concentrated BiNPs were redispersed in aqueous solutions of varying $\mathrm{pH}$ values using 1.0 M phosphoric acid adjusted with $\mathrm{KOH}$ to achieve specified $\mathrm{pH}$ values in the range 2-12; these samples were protected from light. Additionally, two samples were redispersed in water; one was stored in the dark and the other was exposed to sunlight. Visual observation (Figure 2.6 a-c) and UV-visible 
spectroscopy (Figure $2.6 \mathrm{~d}$ ) were used to monitor the stability of the colloids, which was indicated by the maintenance of a dark solution color and the absence of precipitates.

When BiNPs were introduced to the $\mathrm{pH}=2$ solution, the particles immediately dissolved, as evidenced by the loss of solution color and absence of precipitate. Samples in solutions at $\mathrm{pH}$ values of $4,6,7$, and 12 initially retained their color, but fully dissolved to yield colorless solutions within 24 hours; the low $\mathrm{pH}$ results suggest that the acidic environment of a lysosome would decompose these particles in vivo. However, samples redispersed in solutions at $\mathrm{pH}=8$ and 10 were colloidally stable for over one month, as was a control sample diluted in nanopure water. The latter result suggests that phosphate ions may assist degradation over some $\mathrm{pH}$ range, but the effect of ion identity and solution ionic strength on BiNP stability was not further examined.

Photodegradation was examined by preparing two samples of dilute colloidal solutions of BiNPs in glass scintillation vials. One sample was exposed to ambient light, and one sample was protected from light by a single layer of aluminum foil. Both samples were placed side by side in a window, and after 2 weeks the sample exposed to light had notably decreased in color intensity (Figure $2.6 \mathrm{c}$ ). 


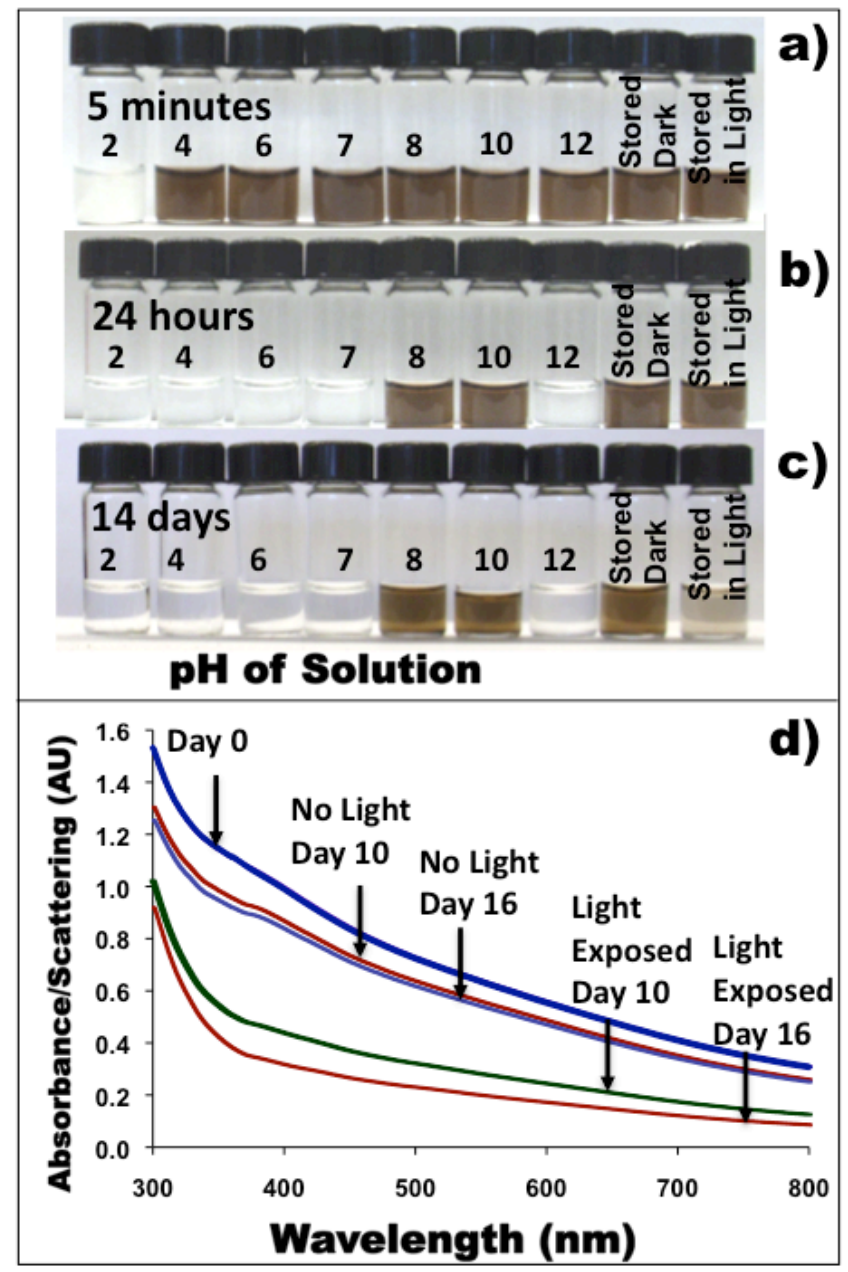

Figure 2.6. Stability of BiNPs in phosphate buffered solutions and exposed to light. (a-c) For pH stability experiments, purified BiNPs were diluted in $1.0 \mathrm{M}$ phosphate solutions and the $\mathrm{pH}$ was adjusted with $\mathrm{KOH}$ to achieve solutions ranging in $\mathrm{pH}$ from 2 to 12; light/dark controls were diluted in water and visually analyzed at the same time points. All vials were examined for solution color and presence or absence of precipitates at time points of (a) 5 min, (b) $24 \mathrm{~h}$, and (c) 14 days. (d) For spectroscopically monitored light stability experiments, purified colloids were diluted in $\mathrm{H}_{2} \mathrm{O}$ and spectra taken at time points of 0,10 , and 14 days. Light-exposed samples were placed in vials in direct sunlight and all other samples were stored in vials wrapped in aluminum foil; absorbance was monitored at $650 \mathrm{~nm}$. 
To spectroscopically monitor for photo-degradation, a dilute solution of BiNPs was initially divided into two cuvettes and the UV-visible spectrum of each was recorded daily over a period of 16 days (Figure $2.6 \mathrm{~d}$ ). One cuvette was wrapped in aluminum foil and both cuvettes were placed in direct sunlight; care was taken not to agitate the samples in between measurements in case settling of particles or precipitate formation occurred. Initially, both samples showed identical broad absorption spectra, covering the entire visible range with shoulders in the absorption spectra apparent around $375 \mathrm{~nm}$. The sample constantly exposed to light lost absorbance intensity uniformly across the visible spectrum, and changed from a dark solution to a light brown solution slowly over the course of several days. Conversely, the sample stored in the dark appeared to be much more stable, as evidenced by a much slower loss of intensity over time. The photo-instability of these particles is unsurprising given their high visible light absorption cross-section and may represent an alternative decomposition and clearance route for elemental BiNPs employed as XCAs.

The BiNP colloids were also tested for temperature stability by freezing and boiling BiNP samples redispersed in nanopure water. BiNP samples diluted in water and heated to boiling for greater than one hour remained colloidally stable, without loss of solution color or formation of precipitates. Additionally, water-dispersed BiNPs were slowly frozen $\left(-20^{\circ} \mathrm{C}\right)$ and rapidly frozen in liquid nitrogen; neither treatment reduced the color intensity or colloidal stability of the 
particle solutions upon thawing. These experiments indicate that the glyco-BiNP colloids are robust over a wide range of temperatures.

\subsection{Conclusion}

The recent popularity of green chemical techniques has put an emphasis on using water as a solvent for the synthesis of nanomaterials, and water solubility and stability are required for nanomaterial X-ray contrast agents. These features are difficult to achieve in oxidatively prone elements such as bismuth. Furthermore, the synthesis of elemental nanomaterials by reduction of cations often requires a strong reducing agent and controlled kinetics to avoid formation of bulk materials and to promote uniform particle growth. Herein, we have described a synthesis method that takes advantage of a sparingly soluble solid to kinetically slow the formation and growth of $\mathrm{Bi}$ nanoparticles in water by controlling the $\mathrm{pH}$, and thus the concentration of $\mathrm{Bi}(\mathrm{III})$ monomer in solution available for reduction. The method allows sufficiently slow growth at modestly basic $\mathrm{pH}$ values $(\mathrm{pH}=8-10)$ to result in isolated and colloidally stable, rather than aggregated and precipitated, $\mathrm{Bi}$ nanoparticles. The glucose polymer dextran is used to surface stabilize the nanoparticles and promote chemical and colloidal stability. The glyco-BiNP can be purified and redispersed in water or in a mildly basic phosphate buffer, and these solutions are then stable for months when stored away from light. 
In summary, chemically and colloidally stable, glyco-BiNPs have been synthesized using a simple, $\mathrm{pH}$-controlled, aqueous method. These high atomic number glyco-BiNP have size, solubility, and stability characteristics suitable for use as nanomaterial X-ray contrast agents. Bismuth is known to be both highly $\mathrm{X}$ ray attenuating and biologically compatibility, which makes elemental bismuth nanoparticles good candidates for in vivo nano-scale XCAs. We anticipate future experimentation with these particles to include toxicity evaluation and determination of X-ray attenuation coefficients at diagnostic X-ray voltages.

\section{References:}

(1) Zhang, Z.; Ying, J. Y.; Dresselhaus, M. S. Bismuth Quantum-Wire Arrays Fabricated by a Vacuum Melting and Pressure Injection Process. Journal of Materials Research 1998, 13, 1745-1748.

(2) Wang, Y. W.; Kim, J. S.; Kim, G. H.; Kim, K. S. Quantum size effects in the volume plasmon excitation of bismuth nanoparticles investigated by electron energy loss spectroscopy. Applied Physics Letters 2006, 88, 143106.

(3) Heremans, J.; Thrush, C. M. Thermoelectric power of bismuth nanowires. Physical Review B 1999, 59, 12579.

(4) Boukai, A.; Xu, K.; Heath, J. R. Size-Dependent Transport and Thermoelectric Properties of Individual Polycrystalline Bismuth Nanowires. Advanced Materials 2006, 18, 864-869. 
(5) Fanfair, D. D.; Korgel, B. A. Bismuth Nanocrystal-Seeded III-V

Semiconductor Nanowire Synthesis. Crystal Growth \& Design 2005, 5, 1971-1976.

(6) Wang, F.; Tang, R.; Kao, J. L.-F.; Dingman, S. D.; Buhro, W. E. Spectroscopic Identification of Tri-n-octylphosphine Oxide (TOPO) Impurities and Elucidation of Their Roles in Cadmium Selenide QuantumWire Growth. Journal of the American Chemical Society 2009, 131, 49834994.

(7) Chockla, A. M.; Harris, J. T.; Korgel, B. A. Colloidal Synthesis of Germanium Nanorods. Chemistry of Materials 2011, 23, 1964-1970.

(8) Rabin, O.; Manuel Perez, J.; Grimm, J.; Wojtkiewicz, G.; Weissleder, R. An X-ray computed tomography imaging agent based on long-circulating bismuth sulphide nanoparticles. Nature Materials 2006, 5, 118-122.

(9) Pan, D.; Roessl, E.; Schlomka, J.; Caruthers, S. D.; Senpan, A.; Scott, M. J.; Allen, J. S.; Zhang, H.; Hu, G.; Gaffney, P. J.; Choi, E. T.; Rasche, V.; Wickline, S. A.; Proksa, R.; Lanza, G. M. Computed Tomography in Color: NanoK-Enhanced Spectral CT Molecular Imaging. Angewandte Chemie 2010, 122, 9829-9833.

(10) Ai, K.; Liu, Y.; Liu, J.; Yuan, Q.; He, Y.; Lu, L. Large-Scale Synthesis of Bi2S3 Nanodots as a Contrast Agent for In Vivo X-ray Computed Tomography Imaging. Advanced Materials 2011, 23, 4886-4891. 
(11) Choi, H. S.; Liu, W.; Misra, P.; Tanaka, E.; Zimmer, J. P.; Ipe, B. I.; Bawendi, M. G.; Frangioni, J. V. Renal Clearance of Nanoparticles. Nature Biotechnol 2007, 25, 1165-1170.

(12) Mammen, M.; Choi, S.; Whitesides, G. M. Polyvalent Interactions in Biological Systems: Implications for Design and Use of Multivalent Ligands and Inhibitors. Angewandte Chemie International Edition 1998, 37, 27542794.

(13) Briand, G. G.; Burford, N. Bismuth compounds and preparations with biological or medicinal relevance. Chemical Reviews 1999, 99, 2601-2658.

(14) Hainfeld, J. F.; Slatkin, D. N.; Focella, T. M.; Smilowitz, H. M. Gold nanoparticles: a new X-ray contrast agent. British Journal of Radiology 2006, 79, 248-253.

(15) Popovtzer, R.; Agrawal, A.; Kotov, N. A.; Popovtzer, A.; Balter, J.; Carey, T. E.; Kopelman, R. Targeted Gold Nanoparticles enable Molecular CT Imaging of Cancer. Nano Letters 2008, 8, 4593-4596.

(16) Eck, W.; Nicholson, A. I.; Zentgraf, H.; Semmler, W.; Bartling, S. Anti-CD4targeted Gold Nanoparticles Induce Specific Contrast Enhancement of Peripheral Lymph Nodes in X-ray Computed Tomography of Live Mice. Nano Letters 2010, 10, 2318-2322.

(17) Free Bismuth Price Charts. http://www.metalprices.com/pubcharts/Public/Bismuth_Price_Charts.asp (accessed August 8, 2011). 
(18) Greenwood, N.N.; Earnshaw, A. In Chemistry of the elements, Second Edition; Pergamon Press: Oxford, UK, 1998; Vol. Arsenic, Antimony and Bismuth.

(19) Cormode, D. P.; Skajaa, T.; Fayad, Z. A.; Mulder, W. J. M. Nanotechnology in Medical Imaging. Arteriosclerosis, Thrombosis, and Vascular Biology 2009, 29, $992-1000$.

(20) Longmire, M.; Choyke, P. L.; Kobayashi, H. Clearance properties of nanosized particles and molecules as imaging agents: considerations and caveats. Nanomedicine (Lond) 2008, 3, 703-717.

(21) Bierer, D. W. Bismuth subsalicylate: history, chemistry, and safety. Reviews of Infectious Diseases 1990, 12 Supplement 1, S3-8.

(22) Sun, H.; Li, H.; Harvey, I.; Sadler, P. J. Interactions of bismuth complexes with metallothionein(II). Journal Biological Chemistry 1999, 274, 2909429101.

(23) Yu, H.; Gibbons, P. C.; Buhro, W. E. Bismuth, tellurium, and bismuth telluride nanowires Journal Materials Chemistry 2004, 14, 595.

(24) Wang, F.; Tang, R.; Yu, H.; Gibbons, P. C.; Buhro, W. E. Size- and ShapeControlled Synthesis of Bismuth Nanoparticles. Chemistry of Materials 2008, 20, 3656-3662.

(25) Richards, V. N.; Shields, S. P.; Buhro, W. E. Nucleation Control in the Aggregative Growth of Bismuth Nanocrystals. Chemistry of Materials 2011, 23, 137-144. 
(26) Wang, Y.; Xia, Y. Bottom-Up and Top-Down Approaches to the Synthesis of Monodispersed Spherical Colloids of Low Melting-Point Metals. Nano Letters 2004, 4, 2047-2050.

(27) Li, J.; Fan, H.; Chen, J.; Liu, L. Synthesis and characterization of poly(vinyl pyrrolidone)-capped bismuth nanospheres. Colloids and Surfaces $A$ : Physicochemical and Engineering Aspects 2009, 340, 66-69.

(28) Wang, F.; Tang, R.; Yu, H.; Gibbons, P. C.; Buhro, W. E. Size- and ShapeControlled Synthesis of Bismuth Nanoparticles. Chemistry of Materials 2008, 20, 3656-3662.

(29) Wang, Y.; Kim, K. S. Large-scale polyol synthesis of single-crystal bismuth nanowires and the role of $\mathrm{NaOH}$ in the synthesis process. Nanotechnology 2008, 19, 265303.

(30) Wang, Y.; Zhao, J.; Zhao, X.; Tang, L.; Li, Y.; Wang, Z. A facile water-based process for preparation of stabilized Bi nanoparticles. Materials Research Bulletin 2009, 44, 220-223.

(31) Irmawati, R.; Noorfarizan Nasriah, M. N.; Taufiq-Yap, Y. H.; Abdul Hamid, S. B. Characterization of bismuth oxide catalysts prepared from bismuth trinitrate pentahydrate: influence of bismuth concentration. Catalysis Today 2004, 93-95, 701-709.

(32) Kragten, J.; Decnop-Weever, L. G.; Gründler, P. Mixed hydroxide complex formation and solubility of bismuth in nitrate and perchlorate medium. Talanta 1993, 40, 485-490. 
(33) Schumb, W. C.; Rittner, E. S. Polymorphism of Bismuth Trioxide1. Journal of the American Chemical Society 1943, 65, 1055-1060.

(34) Wulfsberg, G. Inorganic chemistry; University Science Books, 2000.

(35) Taylor, P.; Lopata, V. J. Some phase relationships between basic bismuth chlorides in aqueous solutions at $25^{\circ} \mathrm{C}$. Canadian Journal of Chemistry $1987,65,2824-2829$.

(36) Kinomura, N.; Kumada, N. Preparation of bismuth oxides with mixed valence from hydrated sodium bismuth oxide. Materials Research Bulletin 1995, 30, $129-134$.

(37) Suzuki, S.; Shimanouchi, T.; Tsuboi, M. Normal vibrations of glycine and deuterated glycine molecules. Spectrochimica Acta 1963, 19, 1195-1208.

(38) Yarema, M.; Kovalenko, M. V.; Hesser, G.; Talapin, D. V.; Heiss, W. Highly Monodisperse Bismuth Nanoparticles and Their Three-Dimensional Superlattices. Journal of the American Chemical Society 2010, 132, $15158-15159$. 


\section{Chapter 3 - Ultra-High Payload Elemental Bismuth Nanoparticle X-ray Contrast Agents: Synthesis, X-ray Opacity, and Biological Compatibility}

\section{Adapted from:}

Manuscript in preparation by: Anna L. Brown, Pratap C. Naha, Harold I. Litt, David P. Cormode, Andrea M. Goforth

\subsection{Abstract}

The development of novel inorganic nanoscale X-ray Contrast Agents (XCA) offer many advantages over currently used intravascular molecular contrast agents, including longer circulation and retention times, lower administration volumes and the potential for site directed imaging. Elemental bismuth nanoparticles (BiNPs) are particularly attractive research level XCAs due to the low cost of bismuth, the high atomic number and high density of bismuth, and the probability that BiNPs will oxidatively decompose to biocompatible bismuth(III) ions at controlled rates. Herein we describe the synthesis of ultrahigh payload BiNPs in 1,2-propane diol using a borane reducing agent and glucose as a biocompatible surface stabilizer. Particles have large inorganic cores ( $74 \mathrm{~nm}$ by TEM) to hydrodynamic size ( $86 \mathrm{~nm}$ by DLS) indicating that the majority of the particle volume is constituted of a contrast generating inorganic core. Both synthetic solvent (1,2-Propane diol) and surfactant (glucose) appear on the BiNP surface, as evidenced by ${ }^{1} \mathrm{H}-\mathrm{NMR}$ and FT-IR spectroscopies. These BiNPs have a high X-ray opacity, measured by quantitative computed 
tomography and a minimal cell type specific cytotoxicty. We discuss these particles as novel nanomaterial XCAs, surface stabilized by known biologically compatible organic molecules, with an ultra-high payload to volume ratio.

\subsection{Introduction}

Nanoscale X-ray Contrast Agents (XCAs) are an attractive new class of potentially non-nephrotoxic contrast materials, which can evade kidney filtration because of large size(1) and offer the prospect of blood pool and site directed Xray imaging. $(2,3)$ The $\mathrm{X}$-ray attenuation coefficient $(\mu)$ of a contrast material is related to atomic number $(Z)$ and density $(\rho)$ by the expression $\mu=\rho Z^{4} / A E^{3}$, where $\mathrm{A}$ is the atomic mass of X-ray absorbing atoms and $\mathrm{E}$ is the incident $\mathrm{X}$-ray energy. Inorganic nanocrystal XCAs are commonly composed of higher ( $Z$ ) atoms than current, clinically applied molecular contrast agents containing iodine $(Z=53)$ and barium $(Z=56)$, and because the attenuation coefficient is dominated by the $Z$ term, elements with higher $Z$ values should therefore offer superior X-ray contrast. Furthermore, the high density of atoms in inorganic nanocrystals, which is far greater than the density of high $Z$ atoms in clinical small molecule XCAs, allows for a high X-ray attenuation per unit volume of contrast material, which is required for CT and site-directed X-ray imaging.(2) The elements bismuth and gold are uniquely suited for use as functional inorganic nanocrystal XCAs, as they have high atomic numbers, high densities, and low reported toxicities. Gold nanoparticles (AuNPs) have previously been 
explored as XCAs, and site-directed imaging, long circulation times, and evasion of kidney filtration have been demonstrated in animal studies.(4-9) However, the high price and high utility of bulk gold (currently approximately $\$ 8,950 / \mathrm{mol}(10)$ ) coupled with some reports of AuNP toxicity arising from bioaccumulation,(11-13) complicate further development and clinical application studies of AuNPs. Bismuth nanoparticles (BiNPs) should be significantly less expensive than AuNPs, (bulk bismuth is currently approximately $\$ 4 / \mathrm{mol}(14)$ ) and potentially less likely to bioaccumulate than AuNPs, and will also have a higher $Z(Z=79 \mathrm{Au}, Z=$ $83 \mathrm{Bi})$, which may result in greater X-ray contrast. Notably, bismuth is the highest $Z$ negligibly radioactive element(15) and uncommonly for a heavy metal the element and its compounds, generally containing bismuth(III) ions, are biologically well tolerated in large quantities when ingested.(16) The fate of bismuth and other nanomaterials when administered intravenously is unknown, but the expecation from limited blood serum data from orally administered bulk bismuth(0) and bismuth(III) compounds is promising for BiNP biological compatibility, but does not account for other possible toxicity concerns. Bismuth(III) compounds are some of the most commonly used over-the-counter medications world-wide, and bismuth(III) plasma concentrations up to $50 \mu \mathrm{g} / \mathrm{mL}$ are generally considered safe,(17) suggesting that the soluble bismuth(III) oxidation product from decomposed BiNPS should pose a minimal toxicity risk.

Bismuth(III) compounds have been safely used medicinally for centuries, despite the poorly characterized formulations of administered compounds and 
minimal understanding of therapeutic mechanism and fate.(18) More recently, reports of bismuth-based nanoparticulate XCAs for blood pool imaging have included $\mathrm{Bi}_{2} \mathrm{~S}_{3}$ nanoparticles stabilized by biocompatible PVP polymer,(19, 20) bismuth ions aggregated into polymer nanoclusters,(21) and $20 \mathrm{~nm}$ PVP coated elemental BiNPs used in 3D cell detection in a microtissue assay. $(22,23)$ Of these examples, the latter represents the more ideal nanoparticle XCA because its $\mathrm{X}$-ray opaque portion is composed entirely of densely packed, high $\mathrm{Z}$ atoms. While the optimal size of inorganic nanocrystals for use as XCAs is not convincingly established, synthetic methods that yield large payload ( $>50 \mathrm{~nm}$ dense cores), morphologically uniform, and biocompatible BiNPs are needed for further exploratory studies. However, relative to other inorganic nanocrystals (e.g., gold, silver, cadmium selenide, iron oxide, etc.), synthetic methods to produce BiNPs are in their infancy.

To date, elemental BiNPs have been prepared via several synthetic routes (see section 1.6 of this thesis) which can be grouped into two main types: 1) non-aqueous routes, where a dissolved bismuth(III) precursor is thermally or chemically reduced in an organic solvent, such as hexadecylamine/toluene cosolvent,(24) cyclohexane,(25) tetrahydrofuran,(26) N,N-dimethylformamide,(22, 27) 1,3-diisopropylbenzene, (28-31) or ethylene glycol,(32-34) sometimes in the presence of an additional surfactant or polymer, and 2) aqueous routes, where a bismuth(III) salt solution or suspension is chemically reduced, generally in the presence of surface stabilizing surfactants or polymers. While aqueous 
preparations are perhaps more desirable for product biocompatibility considerations, non-aqueous preparations have in general resulted in better size and morphology control, which is likely related to the generally poor solubility of bismuth(III) precursors in water.(18, 35-37)

Perhaps the best size and morphology control over BiNPs has been demonstrated in the thermal or chemical, non-aqueous reduction of $\mathrm{Bi}\left(\mathrm{N}\left(\mathrm{SiMe}_{3}\right)_{2}\right)_{3}$ in apolar solvents, $(24,29,30)$ though the resultant products are non-aqueous, and potentially surface coated to some degree with the organic solvents used in synthesis. Thermal polyol reduction reactions of dissolved bismuth(III) in putatively benign polyalcohol solvents, most commonly in ethylene glycol, have been accomplished, but generally result in both poor morphology and size control of the bismuth nanocrystals. Finally, aqueous BiNP preparations are rare and suffer many setbacks in size, size polydispersity and morphology control, due to poor and $\mathrm{pH}$-dependent solubility of bismuth(III) ions in water. Three successful examples of aqueous preparations are our $\mathrm{pH}$-dependent synthesis, which yields small $(20 \mathrm{~nm})$ somewhat polydisperse BiNP cores that are embedded in a large $(60 \mathrm{~nm})$ dextran-polymer shell,(38) the aqueous reduction of bismuth(III) salts by either aqueous hydrazine in the presence of sodium oleate, (39) or sodium-hypophosphite in the presence of tartaric acid and sodium oleate (40) which ultimately yields $25-40 \mathrm{~nm}$ highly hydrophobic particles. For both of these preparations particles appear highly aggregated, suggesting the oleate surfactant yields surface hydrophobic particles. 
Since synthetic methods capable of achieving ultra-high payload, sizeand morphology-controlled, biologically compatible BiNPs ideally suited for use as XCAs have not been reported, we set out to achieve a scalable (in quantity) and controllable (in size and morphology) synthesis for BiNPs that employs biologically benign reagents and results in large (> $50 \mathrm{~nm}$ ), highly X-ray opaque, aqueous BiNP colloids. Herein, we report the synthesis of large $(74 \pm 14 \mathrm{~nm})$ BiNPs by chemical reduction of dissolved bismuth(III) nitrate in 1,2-propanediol (PPD), which uses glucose as a biocompatible small molecule surfactant. Though the synthesis is non-aqueous, PPD is inexpensive, regarded as biocompatible, and is capable of solubilizing bismuth(III) precursors at high concentrations. For BiNPs synthesized at $80^{\circ} \mathrm{C}$, we demonstrate a high clinical X-ray opacity in body phantom and cellular CT imaging studies. Furthermore, BiNPs produced by this method do not result in decreased cell viability with short exposures (1 hour) to either HeLa or macrophage cells, but do result in decreased viability for macrophage cells at long (24 hour) exposures.

\subsection{Experimental and Methods}

\section{Synthesis of BiNPs}

In a $250 \mathrm{~mL}$ beaker, $54 \mathrm{~g} \alpha$-D-glucose, (Acros Organics, >99+\%, anhydrous) was partially dissolved in $84 \mathrm{~mL}$ 1,2-propanediol (PPD, Acros Organics, 99\%) by immersion of the beaker in a temperature-controlled oil bath with mechanical stirring. To this mixture, $4 \mathrm{~mL}$ of a $250 \mathrm{mM}$ bismuth nitrate pentahydrate (Acros 
Organics, 98\%) solution in PPD was added, and the temperature was increased to a synthetic reaction temperature varying between 60 and $100^{\circ} \mathrm{C}$. Borane morpholine (Acros Organics, 97\%) dissolved in PPD (250 mM) was added (12.2 $\mathrm{mL}$ ) to initiate particle formation. The reaction was stirred for 60 seconds after addition of reducing agent and then quenched by pouring the beaker contents into $200 \mathrm{~mL}$ of electrophoretically pure ice water. To obtain larger quantities of particles for biological assays, batches of particles were pooled prior to work up and purification. For syntheses using surfactants other than glucose, an equimolar amount of surfactant was substituted.

\section{Purification of BiNPs}

A $20 \mathrm{~mL}$ aqueous solution of the black BiNPs was diluted in an additional $30 \mathrm{~mL}$ water and centrifuged at $3.0 \mathrm{krcf}$ for 30 minutes. Following centrifugation, particles were dialyzed (SnakeSkin regenerated cellulose dialysis tubing, 10k MWCO) against electrophoretically pure water to remove excess solvent and reaction by-products. Finally the BiNPs were passed through a $450 \mathrm{~nm}$ syringe filter (Chromafil Xtra PA-45/25 polyamide) and either stored at $4{ }^{\circ} \mathrm{C}$ or flash frozen on liquid nitrogen and lyophilized.

\section{BiNP Characterization}

TEM: Transmission Electron Microscopy (TEM) was performed on an FEI Tecnai F-20 TEM operating at $200 \mathrm{kV}$. Purified particles were briefly sonicated and then 
dropcast onto holey carbon Cu supported TEM grids (Ted Pella) and dried at $100^{\circ} \mathrm{C}$ for 1 hour prior to imaging. For the core size distribution of BiNPs synthesized with glucose surfactant at $80{ }^{\circ} \mathrm{C}$, the longest diameter internal to the nanocrystal was measured for 1124 particles.

SEM: Scanning Electron Microscopy (SEM) was performed on a FEI Sirion XL30 FEG SEM. Samples were drop-cast onto an aluminum support stub and sputter coated with gold (PELCO 91000 Sputter Coater) for $60 \mathrm{sec}$.

DLS: Dynamic Light Scattering (DLS) measurements were performed on a Horiba LB-550 dynamic light scattering instrument, and an instrumental algorithm was used to supply the hydrodynamic number distribution. For measurements, freshly syringe filtered samples were dispersed in water and measured at 5 dilutions to ensure size distributions independent of concentration effects. A representative hydrodynamic diameter distribution, as determined by DLS, is shown for BiNPs synthesized at $80^{\circ} \mathrm{C}$ with glucose surfactant. The same sample used to determine core measurements by TEM was used for DLS measurements. XRD: Lyophilized BiNPs were pressed onto a glass support slide and X-ray diffraction (XRD) data were collected in focused beam (Bragg-Brentano) geometry on a Rigaku Ultima IV X-ray diffraction system using graphite monochromatized $\mathrm{Cu} \mathrm{K} \alpha$ radiation. Scans were performed over the angular range $10-70^{\circ} 2 \theta$ at a scan rate of $0.25 \%$ min at room temperature.

FT-IR: Fourier transform infrared (FT-IR) absorbance spectra were collected on a Thermo Scientific Nicolet iS10 spectrophotometer equipped with a single-bounce 
diamond attenuated total reflectance (ATR) attachment. Aqueous or lyophilized BiNPs were drop-cast or pressed with a glass slide onto the ATR crystal to deposit sample films for analysis. Glucose solid and liquid PPD were measured in their neat forms for comparison by placing them in physical contact with the ATR crystal for measurement.

NMR: Lyophlized BiNPs, glucose or PPD was dissolved in $750 \mu \mathrm{L} \mathrm{D}_{2} \mathrm{O}$ (Cambridge Isotope Laboratories, 99.9\%) and measured on a Bruker $600 \mathrm{MHz}-$ ${ }^{1} \mathrm{H}$ AVANCE-III Nuclear Magnetic Resonance (NMR) spectrometer using a standard pulse sequence (Zg30). Spectra were processed using the Bruker TopSpin 2.1 software package.

\section{Inductively Coupled Plasma Optical Emission Spectroscopy (ICP-OES):}

BiNP stocks $(5,10$ and $25 \mu \mathrm{L})$ were each dissolved in $1 \mathrm{~mL}$ of a $1: 1$ mixture of concentrated nitric acid and water. The final volume of each sample was brought to $10 \mathrm{~mL}$ by addition of deionized water. Elemental analysis was performed using ICP-OES (Spectro Genesis ICP) at the Department of Earth and Environmental Science, University of Pennsylvania. For ICP-OES analysis, bismuth analytical standards were purchased from Fisher Scientific (Pittsburgh, USA). The concentration of bismuth was determined for each sample and then multiplied by the dilution factor. The concentrations thus obtained were averaged to give the final bismuth concentration of the samples diluted for quantitative CT measurements and cell viability assays. 


\section{Quantitative CT Measurements}

Solutions of bismuth or iodine ranging from $0-100 \mathrm{mM}$ in concentration were prepared in triplicate in $1.5 \mathrm{~mL}$ micro centrifuge tubes. The samples were BiNPs, $\mathrm{Bi}\left(\mathrm{NO}_{3}\right)_{3}$ or iopamidol, a commercially available iodine-based contrast agent; concentration of BiNPs was determined by ICP-OES. The samples were placed in a plastic rack and the rack was then wrapped in parafilm. The rack was placed on top of another rack and taped to the bottom of a plastic container 24 $\mathrm{cm}$ wide. The container was filled with water to $21 \mathrm{~cm}$ in height, to simulate attenuation effects of a patient. The preparation of these body phantoms has been described previously.(41)

Each set of samples was scanned in the container with a Siemens Definition DS 64-slice clinical CT scanner at 80, 100, 120 and $140 \mathrm{kV}$ and $300 \mathrm{~mA}$ with a matrix size of $512 \times 512$, field of view at $37 \times 37 \mathrm{~cm}$ and a slice thickness of $0.6 \mathrm{~cm}$. The reconstruction kernel used was B30f. Images were analyzed using Osirix 64 bit (v3.7.1). The attenuation values, in Hounsfield Units (HU) for each sample tube was recorded from three different slices and averaged for each concentration. Attenuation rates $(\mathrm{HU} / \mathrm{mM})$ reported herein are the gradients calculated from of the attenuation $(\mathrm{HU})$ versus concentration $(\mathrm{mM})$ plots.

\section{In vitro cytotoxicity assay:}

Cell culture: J774A.1 murine macrophage cells and HeLa human cervix adenocarcinoma cells were purchased from ATCC, Manassas, VA, 
USA. J774A. 1 cells were maintained in a culture medium of DMEM (Dulbecco's Modified Eagle's Medium), while HeLa cells were maintained in EMEM (Eagle's Minimum Essential Medium). In each case the medium was supplemented with $10 \%$ FBS, $45 \mathrm{IU} \mathrm{mL} \mathrm{m}^{-1}$ penicillin, and $45 \mathrm{IU} \mathrm{mL} \mathrm{m}^{-1}$ streptomycin. The cells were incubated at $37{ }^{\circ} \mathrm{C}$ in $5 \% \mathrm{CO}_{2}$. Incubations with BiNPs were performed as previously described.(42) Briefly, both types of cells were seeded from confluent flasks at a 1:1 dilution into six well plates (2 mL/well) and allowed to adhere and normalize for 24 hours. The resulting cell monolayers were then washed with sterile phosphate buffered saline (PBS). BiNPs dispersed at concentrations of 0 , $0.10,0.25$, or $0.50 \mathrm{mg} / \mathrm{mL}$ in the appropriate cell medium were then incubated with the cells. After 1 or 24 hours incubation, the medium was removed and the cells were gently washed three times with PBS to remove non-internalized nanoparticles. Following incubation and washing, HeLa cells were trypsinized, and the J774A.1 cells were collected using cell scrapers. The cells were centrifuged at $800 \mathrm{rpm}$, the supernatant removed and the cell pellets were dispersed in $2 \% \mathrm{v} / \mathrm{v}$ aqueous glutaraldehyde. The cells were allowed to settle into loosely packed pellets for further quantitative CT attenuation measurements. Each experiment was done in triplicate.

\section{MTS ((3-(4,5-dimethylthiazol-2-yl)-5-(3-carboxymethoxyphenyl)-2-(4- sulfophenyl)-2H-tetrazolium) Cell Viability Assay: Cytotoxicity assays were performed in 96-well microplates (Nunc, Denmark) seeded with $100 \mu \mathrm{L}$ of $1 \times 10^{5}$ cells/mL HeLa or J774A.1 cell suspensions. After 24 hours, cells were washed}


with PBS and treated with solutions of BiNPs dispersed in complete culture medium and incubated at $37^{\circ} \mathrm{C}$ in a $5 \% \mathrm{CO}_{2}$ humidified incubator. Aqueous DMSO $(10 \% \mathrm{v} / \mathrm{v})$ was used as the positive control for both cell lines.

Assays were carried out according to manufacturer's instructions. Briefly, media were removed after 1 or 24 hours and the cells were washed gently with sterile PBS, $100 \mu \mathrm{L}$ cell culture medium and $20 \mu \mathrm{L}$ of MTS/ phenazine methosulfate solution. After incubation and washing, the absorbance of each well was measured in a microplate reader at $490 \mathrm{~nm}$ to assess cellular viability. Three independent experiments were performed for each exposure concentration and the percentage of viable cells was calculated relative to untreated cells. Data are presented as mean \pm standard deviation $(n=3)$.

\section{Results}

\subsection{Synthesis of BiNPs}

For site-directed CT imaging applications, ideal contrast agents will have the largest number of highly X-ray attenuating atoms in the smallest volume. For nanoparticle XCAs this ratio will be affected not only by the size of the dense inorganic X-ray opaque core, but also by the size of its X-ray transparent organic surface stabilizing coating. The surface ligands or polymer stabilizers of inorganic nanoparticles, which can be covalently bound, electrostatically bound, or otherwise physically adsorbed, have been reported to greatly affect the response of cells to nanomaterials in vitro $(43,44)$. Thus the careful selection of all starting 
materials is important for achieving biologically compatible, high functionality XCAs. Furthermore, nanoscale XCAs should be size and morphologically uniform to facilitate dose/response predictability, and because large administration volumes of contrast material are required for this application, particles should be convenient, reproducible and inexpensive to prepare.

We recently reported an aqueous synthesis method that produced dextran-coated BiNPs by $\mathrm{NaBH}_{4}$ reduction of a chemically uncharacterized bismuth(III)nitrate suspension in a glycine buffered basic solution.(38) These particles, when synthesized at $\mathrm{pH} 10$, have a number of appealing properties, such as aqueous synthesis, dense elemental bismuth cores, and a biocompatible surface-stabilizing polymer coating. However, these particles were found to have a low X-ray opacity by volume and did not provide good qualitative X-ray contrast in a concentration range that also maintained good colloidal stability. These particles had an average bismuth core diameter of $20 \mathrm{~nm}$ by TEM and a hydrodynamic diameter of $60 \mathrm{~nm}$ by DLS, which, if modeled as three-dimensional spheres, indicates that particles are less than $4 \%$ bismuth by volume. We conclude that poor X-ray opacity of dextran capped BiNPs is due to the large surfactant shell relative to the inorganic bismuth core.

Substitution of the large molecular weight dextran polymer surfactant with lower molecular weight monomeric glucose capping ligand in the previously described aqueous synthesis routinely produced particles with poor and uncontrolled morphology. We attributed this to the generally poor solubility of 
bismuth(III) salts in aqueous solution and thus we sought alternate biocompatible solvents with greater ability to solvate a bismuth(III) monomer. Good monomer solubility should provide better uniformity in particle nucleation and growth, and thus result in greater synthetic control over particle size, size distribution and morphology. We found that polyalcohol solvents, specifically ethylene glycol, glycerol and PPD were capable of forming stable solutions of bismuth(III) ions, and concentrations of up to 2 molar bismuth(III) solutions could be produced before visible precipitation was observed.

The "polyol reaction," which has been used to reduce metal cations to elemental nanoparticles through a solvothermal process, has previously been used to produce BiNPs in a variety of diol and polyol solvents. $(32,33,45-47)$ However, in general, we found particles produced by this method to be oxidatively unstable when dispersed; particles suspended in water aggregate and slowly oxidize to a solid white precipitate. Polyol reactions are poorly characterized reduction reactions that occur at elevated temperatures $\left(>150^{\circ} \mathrm{C}\right)$ and are thought to proceed by reducing metal cations to elemental nanoparticles via oxidation of the polyalcohol. We hypothesized that introduction of a carbohydrate stabilizing surfactant, such as dextran or glucose, could prevent oxidative decomposition of nanomaterials produced in a polyol synthesis. However, inclustion of carbohydrate ligands under typical polyol raction conditions resulted in a highly polymerized solvent/surfactant/particle aggregate, which is difficult to work up further. 


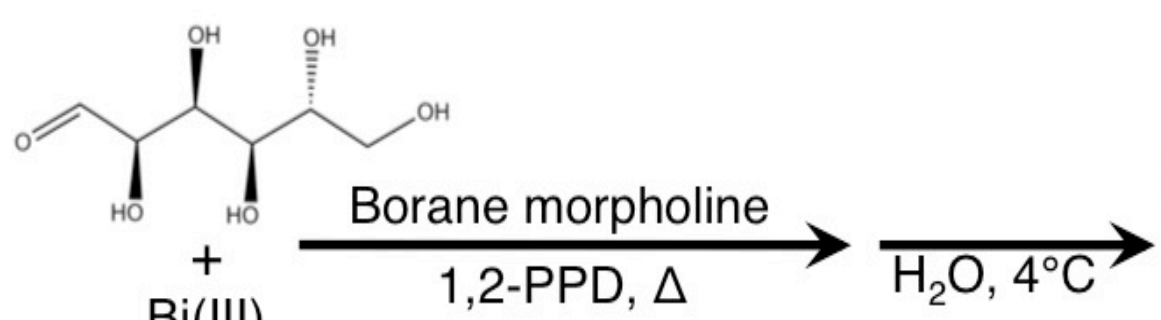

$\mathrm{Bi}(\mathrm{III})$

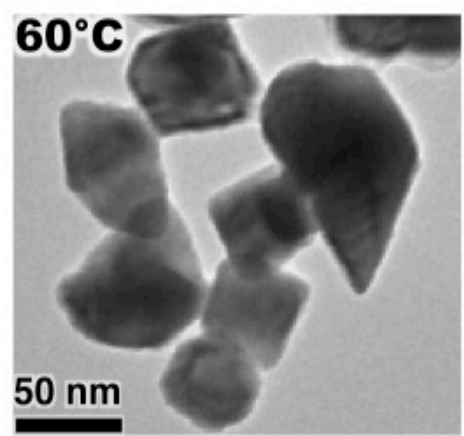

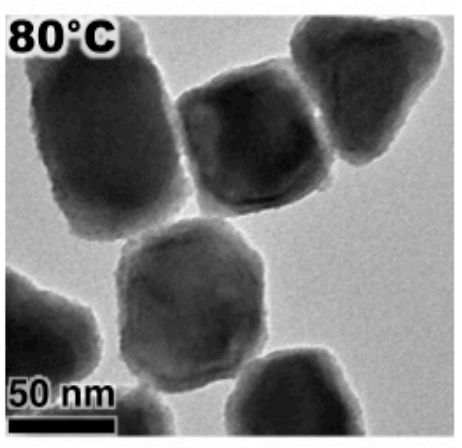

$86 \mathrm{~nm}$ BINP

$74 \mathrm{~nm}$

Scheme 3.1. BiNPs size and morphology dependence on synthetic temperature imaged by TEM. Particles synthesized at $60^{\circ} \mathrm{C}$, (left) appear faceted, crystalline and poly dispersed. Particles synthesized at $80^{\circ} \mathrm{C}$, (middle) provided the best morphology and monodispersity, while particles synthesized at $100^{\circ} \mathrm{C}$, (right) appear rounder and with fewer surface facets, but at also relatively polydispersed.

To avoid elevated temperatures leading to carbohydrate decomposition under the elevated temperatures necessary for a polyol reaction, $\left(>150^{\circ} \mathrm{C}\right)$ we thus used a reducing agent to produce BiNPs in glucose saturated PPD at lower temperatures $\left(60-100^{\circ} \mathrm{C}\right)$. Borane morpholine was chosen as the reducing agent for its kinetically slower reactivity, relative to $\mathrm{NaBH}_{4}$, which should aid in size and morphology control during growth of BiNPs, and for anticipated ease of removal of the expected decomposition products (morpholine and borate) from the aqueous BiNPs. We optimized a BiNP synthesis using PPD solvent because of 
its biocompatibility relative to ethylene glycol, and lower viscosity relative to glycerol (31 mPa.s vs $612 \mathrm{mPa} \cdot \mathrm{s}$ respectively.)(48, 49) PPD is known to be biologically metabolized and is commonly used in food products.(50)

For BiNP synthesis, bismuth(III) nitrate pentahydrate was dissolved in PPD with sonication to produce a fully dissolved bismuth(III) species, and added to a solution of PPD saturated with glucose. Addition of borane morpholine at a specified synthetic temperature initiated the formation of black, colloidal BiNPs. After 60 seconds the entire solution was diluted in ice water to terminate the reaction before further workup. Synthetic temperatures were varied between $60^{\circ} \mathrm{C}$ and $120^{\circ} \mathrm{C}$; generally particles synthesized at higher temperatures $\left(100^{\circ} \mathrm{C}\right)$ produced morphologically more rounded particles, but with a qualitatively greater size distribution, while lower synthetic temperatures $\left(60^{\circ} \mathrm{C}\right)$ produced a greater percentage of highly faceted particles, but qualitatively lower yields of particles were collected during purification (Scheme 3.1). At synthetic temperatures below $60^{\circ} \mathrm{C}$ no reaction was observed, and at temperatures above $120^{\circ} \mathrm{C}$ the carbohydrate surfactant began to polymerize. The trade off between morphology, size, uniformity and yield was optimized for the scope of this work, and further experimentation should result in a more efficient synthesis with lower solvent waste.

To further probe the role glucose plays in particle formation and surface stabilization, the effects of alternate surfactants on nanoparticle morphology were surveyed. The nanoparticle synthesis can be visually monitored by observing the 
formation of a black solution of BiNPs from a colorless bismuth(III) solution upon addition of reducing agent at temperatures between $60-120^{\circ} \mathrm{C}$. In the absence of glucose, no color change was observed upon the addition of borane morpholine to the bismuth(III) starting material in PPD, and this was attributed to the absence of bismuth(0) nanoparticle formation. When sugar alcohols, such as sorbitol and xylitol, replaced glucose in the synthesis, again, no particle formation was observed. These results suggest that a reducing sugar is necessary for particle formation. Fructose (a ketohexose), ribose (an aldopentose), maltose (a reducing disaccharide) all enabled the formation and stabilization of BiNPs, albeit the morphology of the inorganic core was altered substantially (See Appendix B), most likely because the synthesis was optimized for glucose particle formation. The detailed mechanism by which reducing sugars are capable of promoting surface stabilization these BiNPs is beyond the scope of this study, however we anticipate further exploration of this reaction in our ongoing work. 

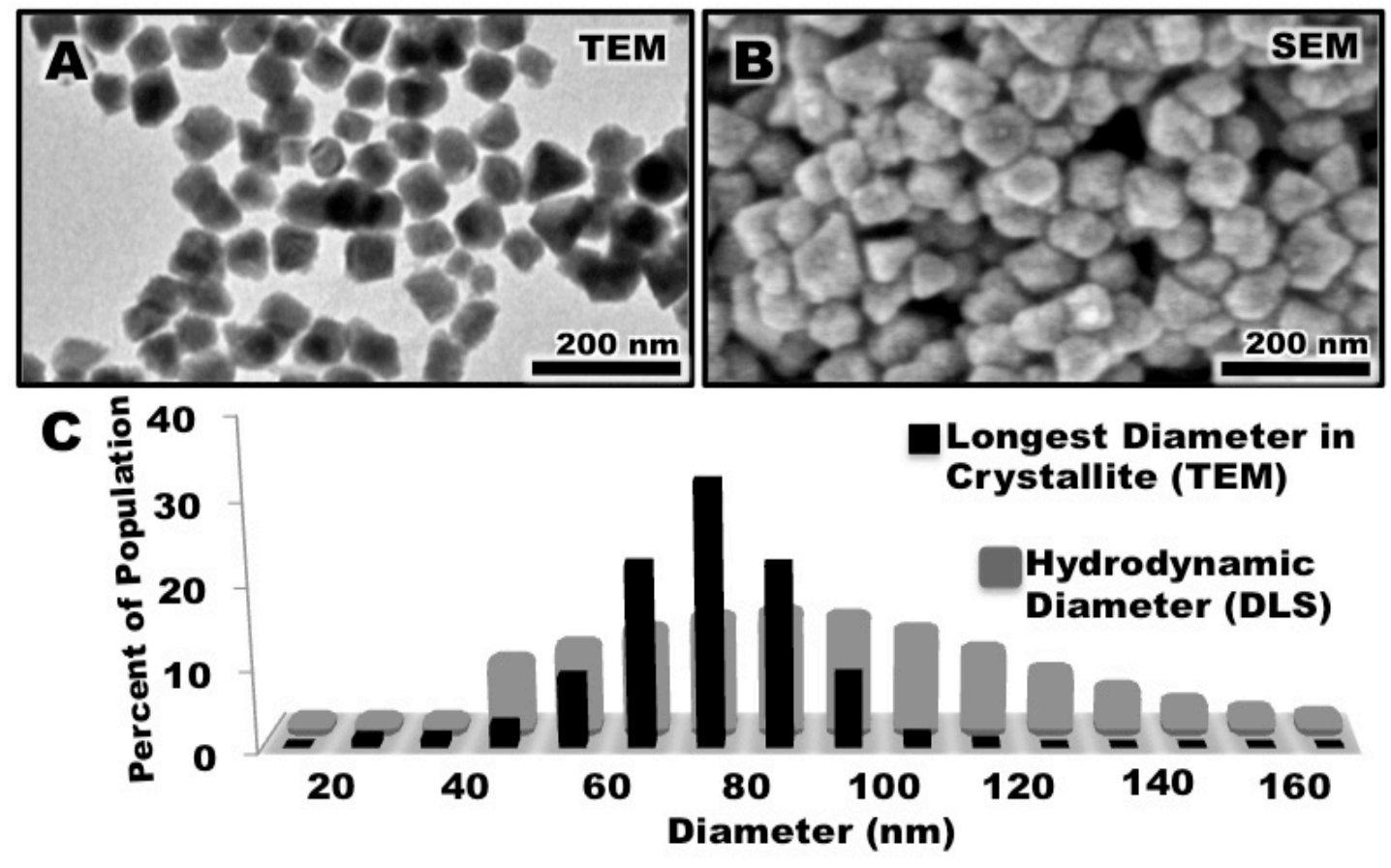

Figure 3.1. Size distributions of inorganic core and overall diameter of BiNPs synthesized in glucose saturated PPD at $80^{\circ} \mathrm{C}$. The longest internal bismuth nanocrystal diameter was measured by TEM ( $\mathrm{A}$; black bars on histogram). BiNP gold sputter coated surfaces were imaged by SEM (B), and the hydrodynamic diameter was measured by DLS, (C; grey bars on histogram).

\subsection{Characterization of Aqueous BiNP Colloids}

BiNPs synthesized at $80^{\circ} \mathrm{C}$ appear by TEM imaging to be discrete, abundant, highly crystalline, and faceted (Figure 3.1 A and B). Because of the anisotropic nanocrystal morphologies observed, a longest diameter (LD) size distribution was obtained by manually measuring the longest apparent internal distance for $>1,000$ nanoparticles by TEM imaging. (Figure 3.1 C) The BiNP core diameters were found to be $74 \pm 14 \mathrm{~nm}$. The LD core size distribution is shown 
(Figure 3.1 C, black histogram) overlaid on a corresponding hydrodynamic diameter size distribution measurement obtained by DLS analysis on the same colloidal sample (Figure 3.1 C, grey histogram). The average hydrodynamic diameter determined by DLS was $86 \mathrm{~nm}$. The SEM image shown, (Figure $3.1 \mathrm{~B}$ ) at the same magnification as the TEM image, (Figure 3.1 A) also shows a similar surface to core diameter ratio for these particles.

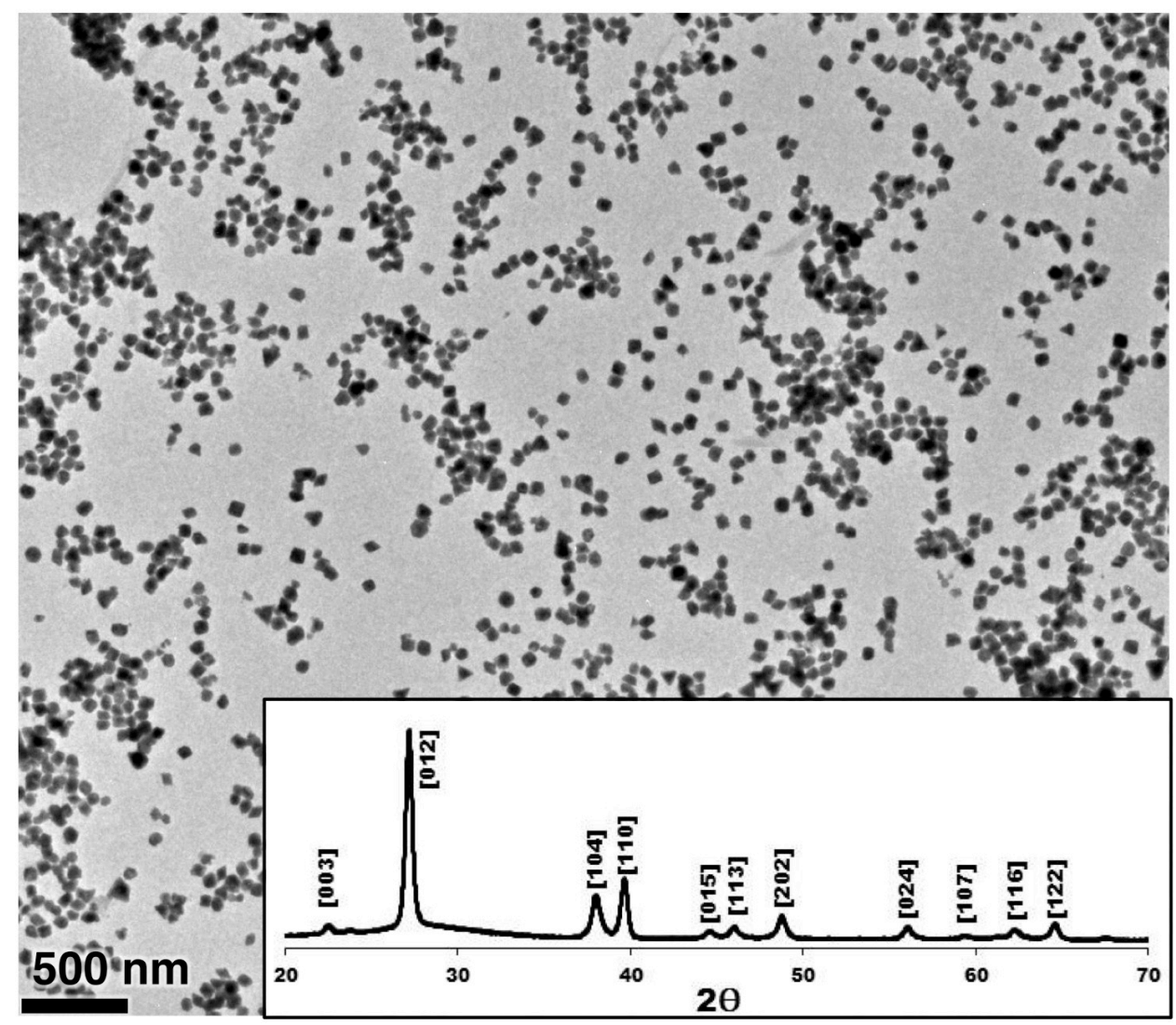

Figure 3.2. TEM image of particle cores. Nanocrystals are discreet and distributed, indicating individual and isolated particles. Powder XRD (inset) of the BiNP sample matches elemental bismuth (JCPDS Card No. 00-044-1246). Miller indices are shown for each reflection peak. 
The similarity in the TEM and DLS size distributions indicates that the majority of the particle volume is constituted of the inorganic nanocrystal core, with only a small portion of the hydrodynamic size attributed to the organic surface stabilizing coating. However size distribution data do not take into account particle anisotropy, which will affect the inorganic core ratio, but are difficult to model for irregular and faceted particles. The $74 \mathrm{~nm}$ core to $86 \mathrm{~nm}$ surface ratio, when modeled in three-dimensional space for volume, indicates that BiNPs are greater than $60 \%$ inorganic bismuth by volume. This is a substantive improvement to our previous synthesized BiNPs that were calculated to be $4 \%$ inorganic bismuth by volume.(38).

Elemental bismuth typically crystallizes in the rhombohedral crystal system (R-3m) and interestingly we observe multiple nanoparticle morphologies by TEM consistent with preferential face growth of rhombohedral nanocrystals (Figure 3.2). Triangles, with $60^{\circ}$ angles, pseudo cubes with near $90^{\circ}$ angles, and hexagons with $120^{\circ}$ angles are observed in Figure 3.2. The PXRD pattern of the BiNPs matches that reported for elemental bismuth (Figure 3.2 inset; JCPDS Card No. 00-044-1246), confirming the rhombohedral, elemental bismuth phase of the particles, however the pattern does not indicate a preferred orientation effects reflected in the calculated vs. experimental peak intensities. While preferential face growth of the nanocrystals during synthesis may occur, as evidenced by irregular particle morphology, no predominant crystal orientation during synthesis or PXRD sample preparation is detected. 


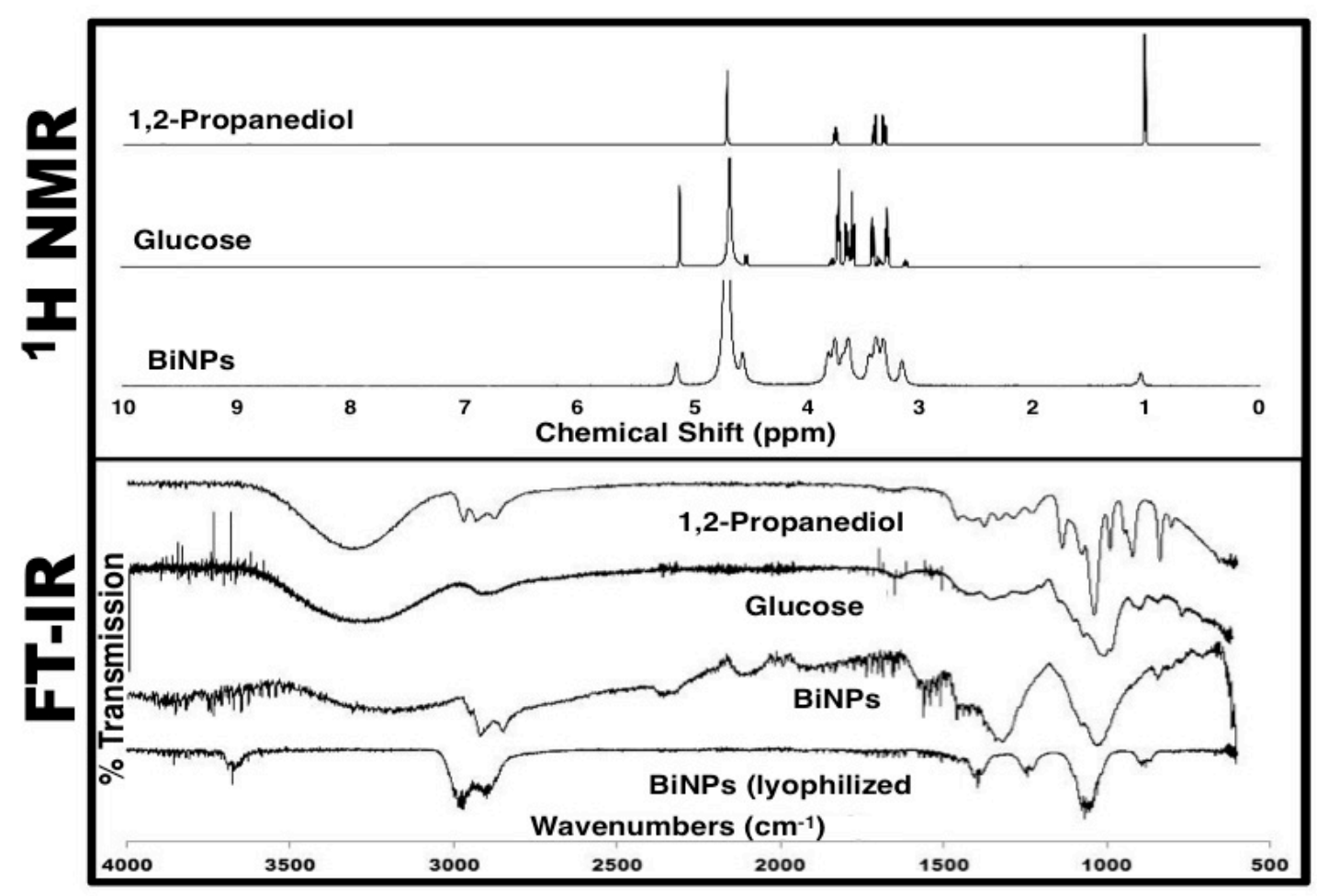

Figure 3.3. Surface characterization of BiNPs by ${ }^{1} H$ NMR and FT-IR. ${ }^{1} H$ NMR spectra indicate both PPD (top) and glucose (middle) are present on the nanoparticle surface. FT-IR measurements indicate a high degree of peak overlap between PPD, glucose and purified particle. Lyophilized BiNPs show a marked decrease in absorbance frequencies, suggesting conformational restrictions of surfactants on the dried nanoparticle surface.

The identity of BiNP surface ligands was determined by ${ }^{1} \mathrm{H}$ NMR and FTIR. The ${ }^{1} \mathrm{H}$ NMR spectrum of washed and dialyzed BiNPs contains peaks from both PPD and glucose, which indicates that both solvent and surfactant are present on the BiNP surface (Figure 3.3). The ${ }^{1} \mathrm{H}$ NMR spectrum of the BiNPs notably contains a peak at $\delta=1.1 \mathrm{ppm}$, consistent with methyl protons from PPD, and a peak at $\delta=5.2 \mathrm{ppm}$ which is consistent with a hydrogen atom bonded to the 
anomeric carbon in glucose. The broad overlapping peaks observed for the BiNPs between $\delta=3-4 \mathrm{ppm}$ are difficult to definitively assign since protons from both PPD and glucose are found in this region. While peak broadening in the BiNP spectrum may be indicative of a slower tumbling rate of the organic molecules in solution when adhered to large inorganic nanoparticles.

Consistent with the ${ }^{1} \mathrm{H}$ NMR results, the FT-IR spectra has features of both from solvent and surfactant, indicating that both are present on the BiNP surface. Specifically the peaks clustered around $1100 \mathrm{~cm}^{-1}$ are assigned to C-O vibrations. C-C vibration absorbance peaks are predicted around $1000 \mathrm{~cm}^{-1}, \mathrm{C}-\mathrm{H}$ stretching around $2900 \mathrm{~cm}^{-1}$ and a broad $\mathrm{O}-\mathrm{H}$ absorbance is observed between $4000-3000 \mathrm{~cm}^{-1}$.(51) Washed and purified BiNPs show a markedly different FTIR spectrum after lyophalization, with a decreased number of peaks between $1500-700 \mathrm{~cm}^{-1}$, in addition to the vibrational $\mathrm{O}-\mathrm{H}$ absorbance. This may be attributable to conformational restrictions of the organic surfactants on the BiNP surfaces, or perhaps to surface molecule cross-linking, which we hypothesize to be the result of a high degree of induced hydrogen bonding that lyophlization imparts by removal of water. The degree of hydrogen bonding, indicating the degree of macromolecular organization, has been previously examined using FTIR spectroscopy to track the degree of crystallinity during cellulose hydrolysis.(52) Here, the altered spectrum suggests restricted movements and vibrations of the organic shell, which indicate increased hydrogen bonding and ordering of carbohydrates on the BiNP surface. 


\subsection{X-ray Contrast and Cytotoxicity Evaluation of Glucose BiNPs}

The X-ray attenuation of BiNPs was measured using a clinical Computed Tomography (CT) scanner and particles loaded into micro centrifuge tubes submerged in a $21 \mathrm{~cm}$ depth water to mimic imaging conditions in a patient. Concentrations of bismuth from 0 to $65 \mathrm{mM}$ were measured at 4 commonly used X-ray tube voltages, namely $80,100,120$, and $140 \mathrm{kV}$, and the CT image obtained for the BiNP solutions is shown. (Figure 3.4 A) The X-ray attenuation in Hounsfield Attenuation (HU) is plotted as a function of bismuth concentration, and shows a linear correlation between bismuth concentration and $\mathrm{HU}$ values. Figure 3.4 B) The X-ray attenuation of bismuth in the form of aqueous BiNPs, relative to that of solvated bismuth(III) nitrate ions and an aqueous iodine standard (iopamidol) is also shown (Figure $3.4 \mathrm{C}$ ) and was obtained by calculating the slope of the attenuation vs. concentration plot for each tube voltage. Both forms of bismuth show significantly higher X-ray attenuation relative to iodine. Interestingly the iodine attenuation rate decreases with increasing X-ray tube voltage, while the bismuth(III) and BiNPs show a relatively voltage insensitive attenuation rate, which may be advantageous for using bismuth in medical imaging application. We postulate that the slightly higher attenuation rates observed for bismuth(III) ions are due to solvent effects; the BiNPs are suspended in water and the bismuth(III) ions are suspended in ethylene glycol. 


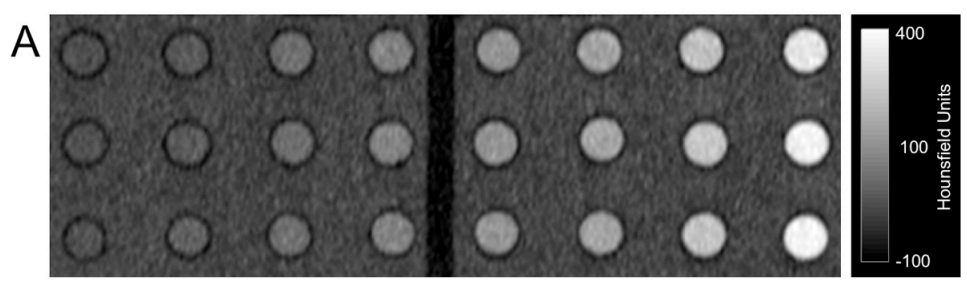

B
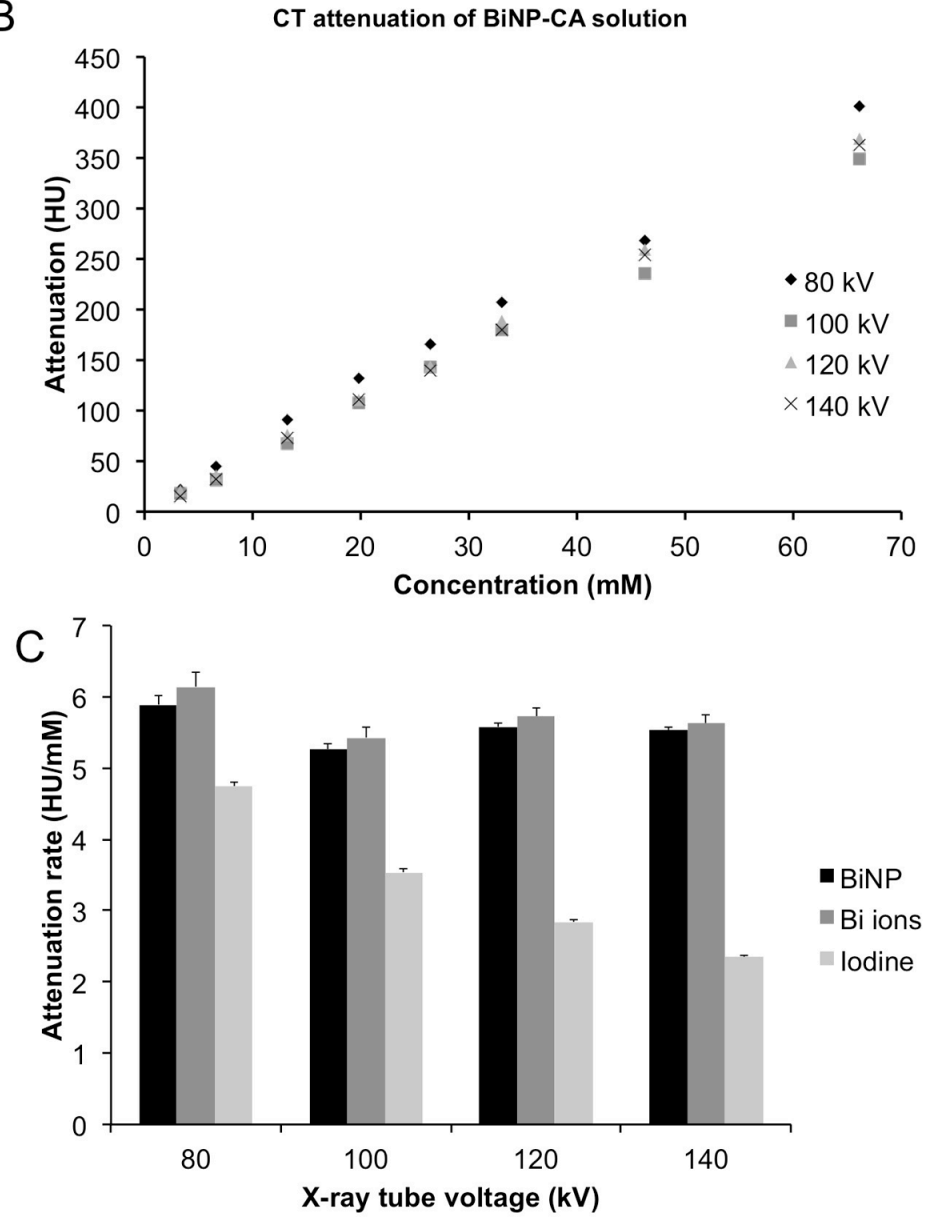

Figure 3.4. CT attenuation evaluation of BiNPs. Solutions varying in concentration between 1-65 mM bismuth were analyzed by ICP-OES and imaged with CT (A). The quantitative attenuation of bismuth in particles as a function of $\mathrm{X}$-ray tube voltages (80-140 kV) is reported in $\mathrm{HU}(\mathrm{B})$. The rate of attenuation as a function of concentration for BiNPs, bismuth(III) ions and iodine were compared at different X-ray tube voltages and are reported in $\mathrm{HU} / \mathrm{mM}$ (C). 

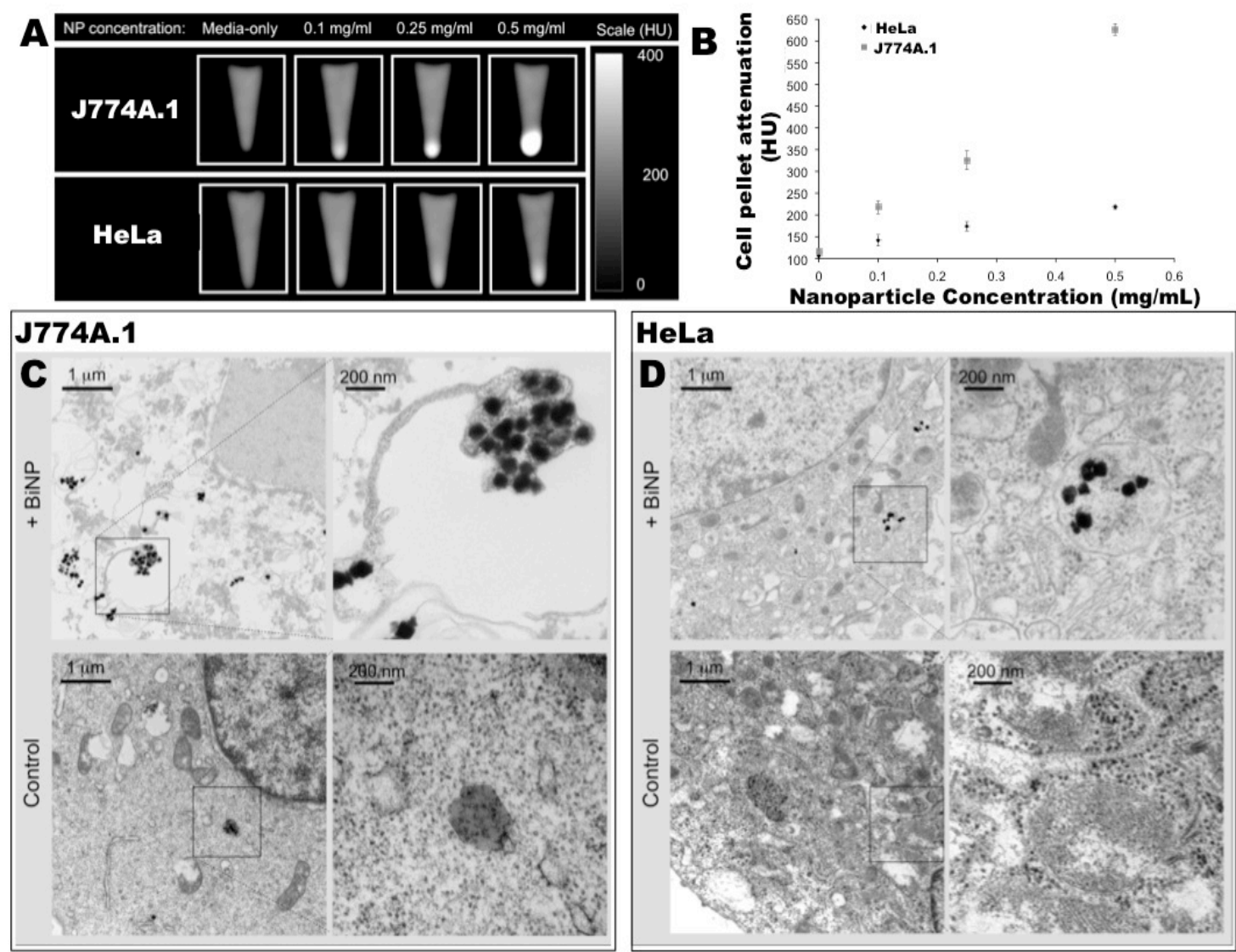

Figure 3.5. Uptake of BiNPs in model cell lines. J774A.1 and HeLa cells were incubated with BiNPs and collected by centrifugation for CT imaging (A) or quantitative attenuation analysis (B). TEM analysis of fixed cells shows that both cell types appear to uptake particles. Vessel formation is clearly observed around particles for both J774A.1 (C) and HeLa cells (D).

Increased biological compatibility is the primary motivation for development of bismuth based XCAs, and as discussed earlier, BiNPs be bleared via different biological pathways than renally filtered molecular iodine XCAs, which will also result in increases retention and circulation times. BiNPs described in this study have a similar $\mathrm{pH}$ oxidative decomposition response as previously described dextran capped particles,(38) namely particles are stable in 
moderately basic phosphate buffered solutions ( $\mathrm{pH}$ values 8-10) but in acidic $(\mathrm{pH}$ values less than 7 ) or extremely basic ( $\mathrm{pH}$ values greater than 10) particles show oxidative decomposition (see Appendix B). The toxicity effects of this $\mathrm{pH}$ dependent stability will be difficult to predict. On one hand the instability may prove advantageous to prevention of bioaccumulation, which is a reported toxicity issue for AuNPs used as XCAs, and on the other hand the decomposition of BiNPs may release damaging or cyto-toxic bismuth species.

Since little is known about the cyto-compatibility of BiNPs, solution stability and cellular response (uptake and viability) studies were performed. In this study two cell types were evaluated for viability in the presence of the BiNPs. HeLa cells were selected for their ubiquitous use in research and murine monocyte macrophage cells (J774A.1) were chosen their known ability to internalize and uptake nanoscale materials. BiNPs were dispersed in culture media $(0.1,0.25$, and $0.5 \mathrm{mg} / \mathrm{mL})$ and incubated for 24 hours before collecting by centrifugation and washing with clean media. CT imaging of the resulting cell pellets shows a substantial increase in attenuation in the macrophage cells incubated with BiNPs, and only a slight increase in the attenuation in the HeLa cells (Figure 3.5 A). Increased uptake of BiNPs by the macrophage cells appears to be concentration dependent, and the X-ray attenuation shown in $\mathrm{HU}$ is plotted against BiNP incubation concentration. (Figure 3.5 B) Cells were also examined by TEM imaging for nanoparticle localization. Fixed macrophage cells show 
visible formation of phagosomes around BiNPs. (Figure $3.5 \mathrm{C}$ ) Engulfment of particles by vessel formation is also noted for HeLa cells. (Figure 3.5 D)

Cell viability was examined using a standard MTS assay that yields quantitative information on cellular enzyme activity. (Figure 3.6) After one hour of incubation with BiNPs no significant decrease in cellular viability is present for either cell type. After 24 hours of incubation with BiNPs, HeLa cells do not show a decrease in viability at BiNP concentrations up to $0.5 \mathrm{mg} / \mathrm{mL}$; however macrophage cell viability drops, with an apparent $\mathrm{LD}_{50}$ of $50 \mu \mathrm{g} / \mathrm{mL}$. The engulfment and absorption of particles by cells suggests that removal of BiNPs, when used as XCAs, will be primarily through the lymphatic system, as these particles are expected to be large enough to avoid filtration through the kidneys. Decreased macrophage cell viability at these elevated concentrations is unsurprising, but does warrant further exploration. The sustained viability of HeLa cells in the presence of BiNPs and the sustained viability of the macrophage cells at 1 hour time points is suggestive of good biological compatibility. Future in vivo studies will further examine biocompatibility and BiNP fate (i.e. clearance) in addition to characterization of the BiNP decomposition products. 

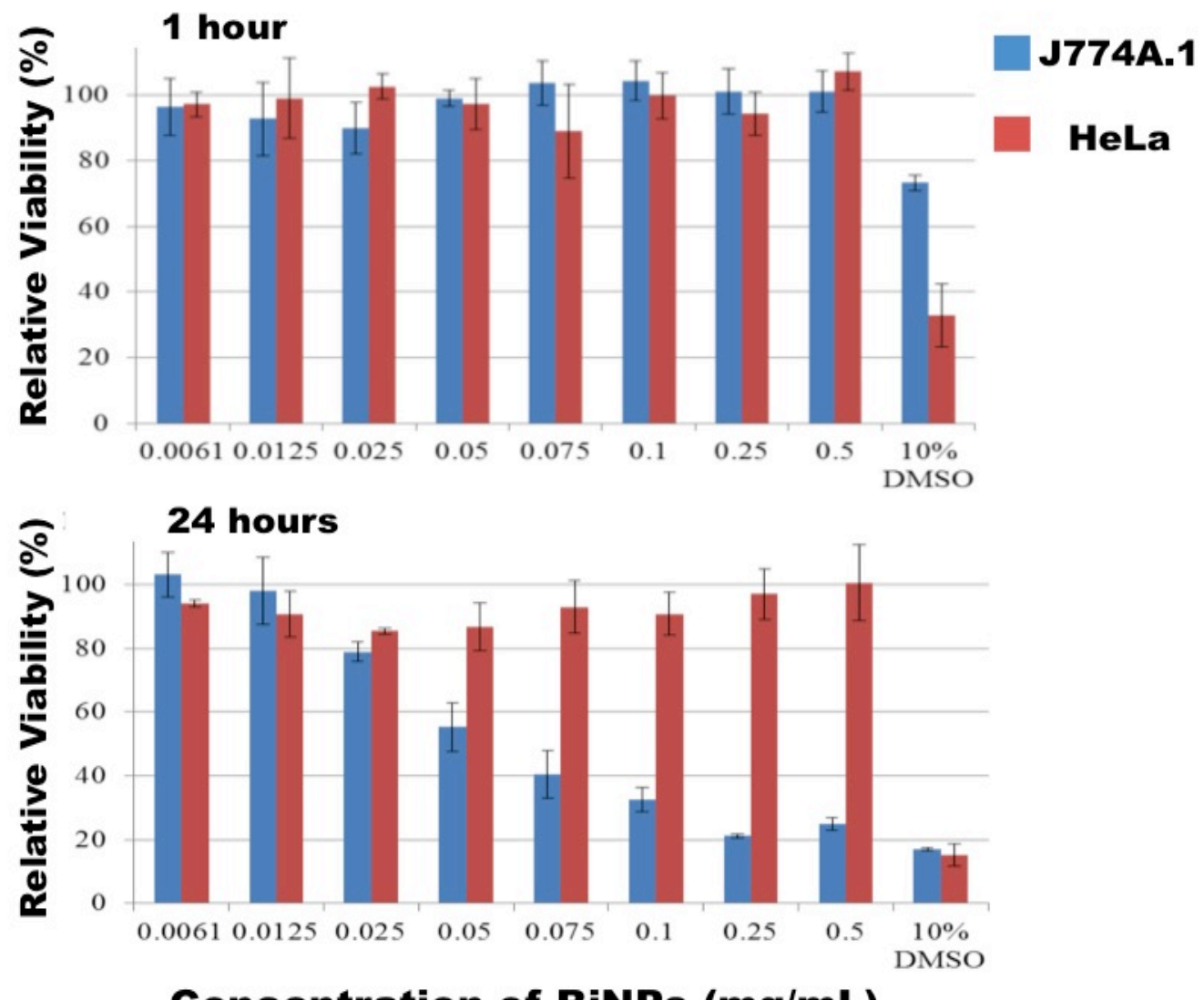

Concentration of BiNPs $(\mathbf{m g} / \mathbf{m L})$

Figure 3.6. Viability of HeLa and J774.A cells incubated with BiNPs. Particle concentrations of $6.1 \mu \mathrm{g} / \mathrm{mL}$ to $0.5 \mathrm{mg} / \mathrm{mL}$ were incubated with cells and viability of cell cultures at 1 hour were assessed relative to unexposed cells. After a 24 hour incubation, HeLa cells show no decrease in cell viability at the highest BiNP concentrations measured and J774A.1 cells show an apparent $L_{50}$ around $50 \mu \mathrm{g} / \mathrm{mL}$. $10 \% \mathrm{v} / \mathrm{v}$ aqueous DMSO was used as a positive control for both cell lines.

\subsection{Conclusion:}

Nanoscale XCAs have been well advocated for their many potential advantages, including potential alternate biological clearance routes and longer 
circulation times relative to molecular XCAs, which may enable blood pool imaging, site directed imaging and decreased administration volumes. BiNPs relative to previously demonstrated AuNPs XCAs are less oxidatively stable and higher in atomic number, which should decrease bioaccumulation while providing increase X-ray contrast. Here we describe and characterize a novel synthetic strategy for the production of stable BiNPs using PPD as an inexpensive biologically compatible and highly coordinating solvent, coupled with an inexpensive glucose surfactant layer. Particles have a large inorganic elemental bismuth core relative to the total hydrodynamic size and ${ }^{1} \mathrm{H}$ NMR and FT-IR both suggest solvent and surfactant are present on the particle surface. Particles show a high X-ray attenuation by CT imaging, and good preliminary biological compatibility studies. These data suggest this will be a new and highly improved research level nanoXCA.

\section{References:}

(1) Choi, H. S.; Liu, W.; Misra, P.; Tanaka, E.; Zimmer, J. P.; Ipe, B. I.; Bawendi, M. G.; Frangioni, J. V. Renal Clearance of Nanoparticles. Nature Biotechnology 2007, 25, 1165-1170.

(2) Jakhmola, A.; Anton, N.; Vandamme, T. F. Inorganic Nanoparticles Based Contrast Agents for X-ray Computed Tomography. Advanced Healthcare Materials 2012, 1, 413-431. 
(3) Debbage, P.; Jaschke, W. Molecular imaging with nanoparticles: giant roles for dwarf actors. Histochemistry and Cellular Biology 2008, 130, 845-875.

(4) Ahn, S.; Jung, S.; Lee, S. Gold Nanoparticle Contrast Agents in Advanced Xray Imaging Technologies. Molecules 2013, 18, 5858-5890.

(5) Cai, Q.-Y.; Kim, S. H.; Choi, K. S.; Kim, S. Y.; Byun, S. J.; Kim, K. W.; Park, S. H.; Juhng, S. K.; Yoon, K.-H. Colloidal Gold Nanoparticles as a Blood-Pool Contrast Agent for X-ray Computed Tomography in Mice. Investigative Radiology 2007, 42, 797-806.

(6) Chien, C.-C.; Chen, H.-H.; Lai, S.-F.; Hwu, Y.; Petibois, C.; Yang, C. S.; Chu, Y.; Margaritondo, G. X-ray imaging of tumor growth in live mice by detecting gold-nanoparticle-loaded cells. Scientific Reports 2012, 2.

(7) Eck, W.; Nicholson, A. I.; Zentgraf, H.; Semmler, W.; Bartling, S. Anti-CD4targeted Gold Nanoparticles Induce Specific Contrast Enhancement of Peripheral Lymph Nodes in X-ray Computed Tomography of Live Mice. Nano Letters 2010, 10, 2318-2322.

(8) Hainfeld, J. F.; Smilowitz, H. M.; O'Connor, M. J.; Dilmanian, F. A.; Slatkin, D. N. Gold nanoparticle imaging and radiotherapy of brain tumors in mice. Nanomedicine 2012, 1-9.

(9) Xu, C.; Tung, G. A.; Sun, S. Size and Concentration Effect of Gold Nanoparticles on X-ray Attenuation As Measured on Computed Tomography. Chemistry Materials 2008, 20, 4167-4169. 
(10) Live Gold, Silver, Platinum, Palladium Quote Spot Price Chart - Kitco. http://www.kitco.com/market/ (accessed June 6, 2013).

(11) Lasagna-Reeves, C.; Gonzalez-Romero, D.; Barria, M. A.; Olmedo, I.; Clos, A.; Sadagopa Ramanujam, V. M.; Urayama, A.; Vergara, L.; Kogan, M. J.; Soto, C. Bioaccumulation and toxicity of gold nanoparticles after repeated administration in mice. Biochemical and Biophysical Research Communications 2010, 393, 649-655.

(12) Khlebtsov, N.; Dykman, L. Biodistribution and toxicity of engineered gold nanoparticles: a review of in vitro and in vivo studies. Chemical Society Review 2011, 40, 1647-1671.

(13) Pan, Y.; Leifert, A.; Ruau, D.; Neuss, S.; Bornemann, J.; Schmid, G.; Brandau, W.; Simon, U.; Jahnen-Dechent, W. Gold Nanoparticles of Diameter $1.4 \mathrm{~nm}$ Trigger Necrosis by Oxidative Stress and Mitochondrial Damage. Small 2009, 5, 2067-2076.

(14) Bismuth metal prices, news, charts and historical prices. http://www.metalprices.com/metal/bismuth (accessed June 6, 2013).

(15) Greenwood, N.N.; Earnshaw, A. In Chemistry of the elements, Second Edition; Pergamon Press: Oxford, UK, 1998; Vol. Arsenic, Antimony and Bismuth.

(16) Sano, Y.; Satoh, H.; Chiba, M.; Okamoto, M.; Serizawa, K.; Nakashima, H.; Omae, K. Journal of Occupational Health 2005, 47, 293-298. 
(17) Serfontein, W. J.; Mekel, R. Bismuth toxicity in man II. Review of bismuth blood and urine levels in patients after administration of therapeutic bismuth formulations in relation to the problem of bismuth toxicity in man. Research Communications in Chemical Pathology and Pharmacology 1979, 26, 391-411.

(18) Briand, G. G.; Burford, N. Bismuth compounds and preparations with biological or medicinal relevance. Chemical Reviews 1999, 99, 2601-2658.

(19) Rabin, O.; Manuel Perez, J.; Grimm, J.; Wojtkiewicz, G.; Weissleder, R. An X-ray computed tomography imaging agent based on long-circulating bismuth sulphide nanoparticles. Nature Materials 2006, 5, 118-122.

(20) Kinsella, J. M.; Jimenez, R. E.; Karmali, P. P.; Rush, A. M.; Kotamraju, V. R.; Gianneschi, N. C.; Ruoslahti, E.; Stupack, D.; Sailor, M. J. X-Ray Computed Tomography Imaging of Breast Cancer by using Targeted Peptide-Labeled Bismuth Sulfide Nanoparticles. Angewandte Chemie International Edition.

(21) Pan, D.; Roessl, E.; Schlomka, J.; Caruthers, S. D.; Senpan, A.; Scott, M. J.; Allen, J. S.; Zhang, H.; Hu, G.; Gaffney, P. J.; Choi, E. T.; Rasche, V.; Wickline, S. A.; Proksa, R.; Lanza, G. M. Computed Tomography in Color: NanoK-Enhanced Spectral CT Molecular Imaging. Angewandte Chemie 2010, 122, 9829-9833. 
(22) Luo, Y.; Wang, C.; Hossain, M.; Qiao, Y.; Ma, L.; An, J.; Su, M. ThreeDimensional Microtissue Assay for High-Throughput Cytotoxicity of Nanoparticles. Analitical Chemistry 2012, 84, 6731-6738.

(23) Hossain, M.; Luo, Y.; Sun, Z.; Wang, C.; Zhang, M.; Fu, H.; Qiao, Y.; Su, M. X-ray enabled detection and eradication of circulating tumor cells with nanoparticles. Biosensors and Bioelectronics 2012, 38, 348-354.

(24) Yarema, M.; Kovalenko, M. V.; Hesser, G.; Talapin, D. V.; Heiss, W. Highly Monodisperse Bismuth Nanoparticles and Their Three-Dimensional Superlattices. Journal of the American Chemical Society 2010, 132, $15158-15159$.

(25) Fang, J.; Stokes, K. L.; Wiemann, J.; Zhou, W. Nanocrystalline bismuth synthesized via an in situ polymerization-microemulsion process. Materials Letters 2000, 42, 113-120.

(26) Warren, S. C.; Jackson, A. C.; Cater-Cyker, Z. D.; DiSalvo, F. J.; Wiesner, U. Nanoparticle Synthesis via the Photochemical Polythiol Process. Journal of the American Chemical Society 2007, 129, 10072-10073.

(27) Wang, Y. W.; Hong, B. H.; Kim, K. S. Size Control of Semimetal Bismuth Nanoparticles and the UV-Visible and IR Absorption Spectra. Journal of Physical Chemistry B 2005, 109, 7067-7072.

(28) Wang, F.; Buhro, W. E. An easy shortcut synthesis of size-controlled bismuth nanoparticles and their use in the SLS growth of high-quality colloidal cadmium selenide quantum wires. Small 2010, 6, 573-581. 
(29) Richards, V. N.; Shields, S. P.; Buhro, W. E. Nucleation Control in the Aggregative Growth of Bismuth Nanocrystals. Chemistry of Materials 2011, 23, 137-144.

(30) Wang, F.; Tang, R.; Yu, H.; Gibbons, P. C.; Buhro, W. E. Size- and ShapeControlled Synthesis of Bismuth Nanoparticles. Chemistry of Materials 2008, 20, 3656-3662.

(31) Schulz, S.; Heimann, S.; Wölper, C.; Assenmacher, W. Synthesis of Bismuth Pseudocubes by Thermal Decomposition of Bi2Et4. Chemistry of Materials 2012, 24, 2032-2039.

(32) Wang, Y.; Kim, K. S. Large-scale polyol synthesis of single-crystal bismuth nanowires and the role of $\mathrm{NaOH}$ in the synthesis process. Nanotechnology 2008, 19, 265303.

(33) Goia, C.; Matijević, E.; Goia, D. V. Preparation of Colloidal Bismuth Particles in Polyols. Journal of Materials Research 2011, 20, 1507-1514.

(34) Wang, Y.; Xia, Y. Bottom-Up and Top-Down Approaches to the Synthesis of Monodispersed Spherical Colloids of Low Melting-Point Metals. Nano Letters 2004, 4, 2047-2050.

(35) Sadler, P. J.; Li, H.; Sun, H. Coordination chemistry of metals in medicine: target sites for bismuth. Coordination Chemistry Reviews 1999, 185-186, 689-709. 
(36) Sun, H.; Li, H.; Harvey, I.; Sadler, P. J. Interactions of bismuth complexes with metallothionein(II). Journal of Biological Chemistry 1999, 274, 2909429101.

(37) Phillips, H. A.; Eelman, M. D.; Burford, N. Cooperative influence of thiolate ligands on the bio-relevant coordination chemistry of bismuth. Journal of Inorganic Biochemistry 2007, 101, 736-739.

(38) Brown, A. L.; Goforth, A. M. pH-Dependent Synthesis and Stability of Aqueous, Elemental Bismuth Glyconanoparticle Colloids: Potentially Biocompatible X-ray Contrast Agents. Chemistry of Materials 2012.

(39) Wang, Y.; Zhao, J.; Zhao, X.; Tang, L.; Li, Y.; Wang, Z. A facile waterbased process for preparation of stabilized Bi nanoparticles. Materials Research Bulletin 2009, 44, 220-223.

(40) Ma, D.; Zhao, J.; Zhao, Y.; Hao, X.; Li, L.; Zhang, L.; Lu, Y.; Yu, C. Synthesis of bismuth nanoparticles and self-assembled nanobelts by a simple aqueous route in basic solution. Colloids and Surfaces $A$ :

Physicochemical and Engineering Aspects 2012, 395, 276-283.

(41) Galper, M. W.; Saung, M. T.; Fuster, V.; Roessl, E.; Thran, A.; Proksa, R.; Fayad, Z. A.; Cormode, D. P. Effect of computed tomography scanning parameters on gold nanoparticle and iodine contrast. Investigative Radiology 2012, 47, 475-481.

(42) Cormode, D. P.; Skajaa, T.; van Schooneveld, M. M.; Koole, R.; Jarzyna, P.; Lobatto, M. E.; Calcagno, C.; Barazza, A.; Gordon, R. E.; Zanzonico, P.; 
Fisher, E. A.; Fayad, Z. A.; Mulder, W. J. M. Nanocrystal Core High-Density Lipoproteins: A Multimodality Contrast Agent Platform. Nano Letters 2008, 8, 3715-3723.

(43) Hirn, S.; Semmler-Behnke, M.; Schleh, C.; Wenk, A.; Lipka, J.; Schäffler, M.; Takenaka, S.; Möller, W.; Schmid, G.; Simon, U.; Kreyling, W. G. Particle size-dependent and surface charge-dependent biodistribution of gold nanoparticles after intravenous administration. European Journal of Pharmaceutics and Biopharmaceutics 2011, 77, 407-416.

(44) Alexis, F.; Pridgen, E.; Molnar, L. K.; Farokhzad, O. C. Factors Affecting the Clearance and Biodistribution of Polymeric Nanoparticles. Molecular Pharmaceutics 2008, 5, 505-515.

(45) Wang, J.; Wang, X.; Peng, Q.; Li, Y. Synthesis and Characterization of Bismuth Single-Crystalline Nanowires and Nanospheres. Inorganic Chemistry 2004, 43, 7552-7556.

(46) Wang, W. Z.; Poudel, B.; Ma, Y.; Ren, Z. F. Shape Control of Single Crystalline Bismuth Nanostructures. The Journal of Physical Chemistry B 2006, 110, 25702-25706.

(47) Cheng, G.; Wu, J.; Xiao, F.; Yu, H.; Lu, Z.; Yu, X.; Chen, R. Synthesis of bismuth micro- and nanospheres by a simple refluxing method. Materials Letters 2009, 63, 2239-2242. 
(48) Segur, J. B.; Oberstar, H. E. Viscosity of Glycerol and Its Aqueous

Solutions. Industrial and Engineering Chemistry Research. 1951, 43, 21172120.

(49) Saleh, M. A.; Begum, S.; Begum, S. K.; Begum, B. A. Viscosity of Dilute Aqueous Solutions of Some Diols. Physics and Chemistry of Liquids 1999, 37, 785-801.

(50) Huff, E. The metabolism of 1,2-propanediol. Biochimica et Biophysica Acta $1961,48,506-517$.

(51) El-Haes, H.; Leon, A. de; Jalbout, A. F.; Alaam, M.; Ibrahim, M. Analysis of the structure and vibrational spectra of glucose and fructose. Eclética Química 2006, 31, 15-21.

(52) Oh, S. Y.; Yoo, D. I.; Shin, Y.; Seo, G. FTIR analysis of cellulose treated with sodium hydroxide and carbon dioxide. Carbohydrate Research 2005, $340,417-428$. 


\section{Chapter 4 - Inexpensive, Aerobic Synthesis of Hydrophobic Organoamine Bismuth Nanoparticles}

\section{Adapted from:}

Manuscript in Preparation by: Anna L. Brown, William Alexander Merrill, Rebecca Chitkowski and Andrea M. Goforth

\subsection{Abstract}

We report a simple aerobic synthetic method for the production of size and morphologically uniform bismuth nanoparticles. Spherical bismuth particles result from the reduction of dissolved bismuth triiodide in a hexadecylamine/hexadecane solvent mixture at $200{ }^{\circ} \mathrm{C}$; all reagents are commercially available and inexpensive. The hexadecylamine serves as solvent, reducing agent, and surface stabilizing ligand, and hexadecane serves as a cosolvent. The organoamine-coated bismuth nanoparticles were characterized by TEM, SEM, and FT-IR spectroscopy.

\subsection{Introduction to Bismuth Nanoparticle Organoamine Synthesis}

The potential uses and applications of nanomaterials are inspiring new fields in science, but testing and exploiting these new materials are often inhibited by synthetic limitations. Bismuth nanoparticles hold great promise for future technologies; as examples the bulk semi-metalloid element has an altered electrical conductivity on its nanoscale surface, which is interesting for 
thermoelectric materials(1-5) and both high atomic number and biocompatibility for medical X-ray attenuation applications. $(6,7)$

Synthesis of morphologically uniform bismuth nanoparticles by previously published methods have generally used air-sensitive precursors, specifically bismuth(III) trimethylsilylamide.(8-10) The advantage of this precursor as a bismuth source for nanoparticle synthesis is its good solubility in nonpolar solvents, which facilitates the solubility of discrete ions for reduction, nucleation, and growth of homogenous particles. However air sensitivity makes preparations using this precursor confined to well equipped and specialized laboratories. Furthermore high time and reagent cost and low particle yield from these specialized precursors can make further application focused experimentation financially limiting. Thus a molecular bismuth precursor which is aerobically stable, inexpensive, and soluble for building nanoparticles is appealing for both basic and applied research. Unfortunately the generally poor solubility of bismuth(III) compounds makes finding a precursor for nanoparticle synthesis difficult. Bismuth readily forms polynuclear ionic species and insoluble precipitates with a wide range of halides and oxides,(11) which, if used in nanoparticle synthesis, can lead to inhomogeneity in nanocrystal morphology. In order to develop a versatile bismuth nanoparticle synthesis using a low cost, soluble precursor, we adopted the Osterloh organoamine method for synthesis of noble metal nanoparticles,(12) using an iodobismuth precursor and hexadecylamine as co-solvent, surfactant, and reducing agent. lodobismuth 
anions have been synthesized under a variety of conditions and have variable stoichometry, but can exist as discrete and isolatable clusters. $(13,14)$

As bismuth structures under $50 \mathrm{~nm}$ are thought to undergo a semimetal to metal transition, the electronic state and atomic arrangement at the bismuth nano surface are complicated but have a number of applications, most notably for thermoelectric materials.(15) Thus a synthetic method that yields particles with selected ligands, or available for ligand exchange, $(4,10)$ should enable a further understanding of bismuth surface electronics. We posit that bismuth nanoparticle surfaces are electron deficient because of the termination of the crystal lattice, but are not overall charged. Thus dative bond stabilization of these surfaces from an electron dense organic molecule with a neutral charge should be favored. We use hexadecylamine as a high boiling solvent that is both hydrophobic and has a primary amine available for reduction of the bismuth cations. The amine additionally acts as an electron-dense, surface-stabilizing ligand. Particles synthesized by this method are relatively uniform and should enable further experimentation for bismuth surface electronic studies and ligand exchange .

\subsection{Synthesis and Methods}

In order to form a soluble iodobismuth precursor, $\mathrm{Bil}_{3}(2.5 \mathrm{mg} / \mathrm{mL})$ in hexadecylamine was heated at $100^{\circ} \mathrm{C}$ for 1 hour to yield an optically clear, bright orange solution. To synthesize nanoparticles, $4.5 \mathrm{~mL}$ of hexadecylamine, or a 
mixture of hexadecylamine and hexadecane (1:1 or 1:3), were heated together to $200{ }^{\circ} \mathrm{C}$ in a beaker submerged in a hot oil bath. Six micromoles of the iodobismuth precursor $(500 \mu \mathrm{L})$ was added to this solution with rapid stirring and the solution darkened from a light amber to a dark red and finally black. Particles were allowed to grow and stabilize for 5 minutes at synthetic temperature. To form spherical particles, solutions were rapidly cooled by removing the beaker from the hot oil bath and adding $15 \mathrm{~mL}$ of $4^{\circ} \mathrm{C}$ toluene. To form highly faceted hexagons and nanostars, $500 \mu \mathrm{L}$ of a $5 \mathrm{mg} / \mathrm{mL} \mathrm{Bil}_{3}$ bismuthiodo cluster prepared as described above was reacted with $4.5 \mathrm{~mL}$ hexadecylamine (hexagons) or $281 \mu \mathrm{L}$ hexadecylamine and $4.2 \mathrm{~mL}$ hexadecane (nanostars) at $200{ }^{\circ} \mathrm{C}$ for 10 minutes. For hexagon synthesis, the reaction solution was removed from heat and allowed to cool to room temperature without stirring. Nanostar morphologies were obtained by removing an aliquot of the reaction solution in a glass Pasteur pipette and immersing the solution into iced toluene.

All particle solutions were then centrifuged at $4.4 \mathrm{kRPM}$ for $20 \mathrm{~min}$ and twice washed in $3 \mathrm{~mL}$ cyclohexane followed by brief sonication to disperse the particles and re-collected by centrifugation to remove the other organic byproducts of the reduction. Particles were either air dried or resuspended in cyclohexane for storage. 


\section{TEM}

Transmission Electron Microscopy (TEM) was performed on an FEI Tecnai F-20 TEM operating at $200 \mathrm{kV}$. Particles suspended in cyclohexane were briefly sonicated and dropcast onto holey carbon Cu supported TEM grids (Ted Pella) and dried at $150{ }^{\circ} \mathrm{C}$ for greater than 2 hours prior to imaging.

\section{SEM}

Particles suspended in cyclohexane were dropcast on aluminum SEM stubs and allowed to air dry in layers. Stubs were sputter coated with gold (PELCO 91000 Sputter Coater) for 40 seconds, and imaged on an FEI Sirion XL30 FEG SEM at $3 \mathrm{keV}$.

\section{FT-IR}

Fourier transform infrared (FT-IR) spectroscopy was performed on a Thermo Scientific Nicolet iS10 spectrophotometer equipped with a single-bounce diamond ATR attachment. For analysis, particles were suspended in cyclohexane, dropped onto the ATR crystal, and solvent was evaporated as necessary using a heat gun.

\subsection{Results and Discussion}

Bismuth nanoparticles were synthesized by a thermal reduction of bismuth iodide in the presence of hexadecylamine and hexadecane. Previous 
reports of oleylamine as a solvent/reducing agent/surfactant for the synthesis of nanoparticles have been reported,(16-19) but the cost limitations for large quantities of high purity oleylamine for synthesis led us to explore alternative organoamines. Hexadecylamine is structurally similar to oleylamine, and importantly has a similarly high boiling port $\left(330^{\circ} \mathrm{C}\right.$ vs $364^{\circ} \mathrm{C}$, respectively) that enables an ambient pressure and high temperature synthesis. However hexadecylamine is synthetically derived which allows reagents at higher purification and a lower cost to be available. Hexadecylamine is a solid at room temperature (mp. $45^{\circ} \mathrm{C}$ ) and this makes laboratory work more difficult, but inconsistent impurities and the high cost of oleylamine outweigh its synthetic ease of use.

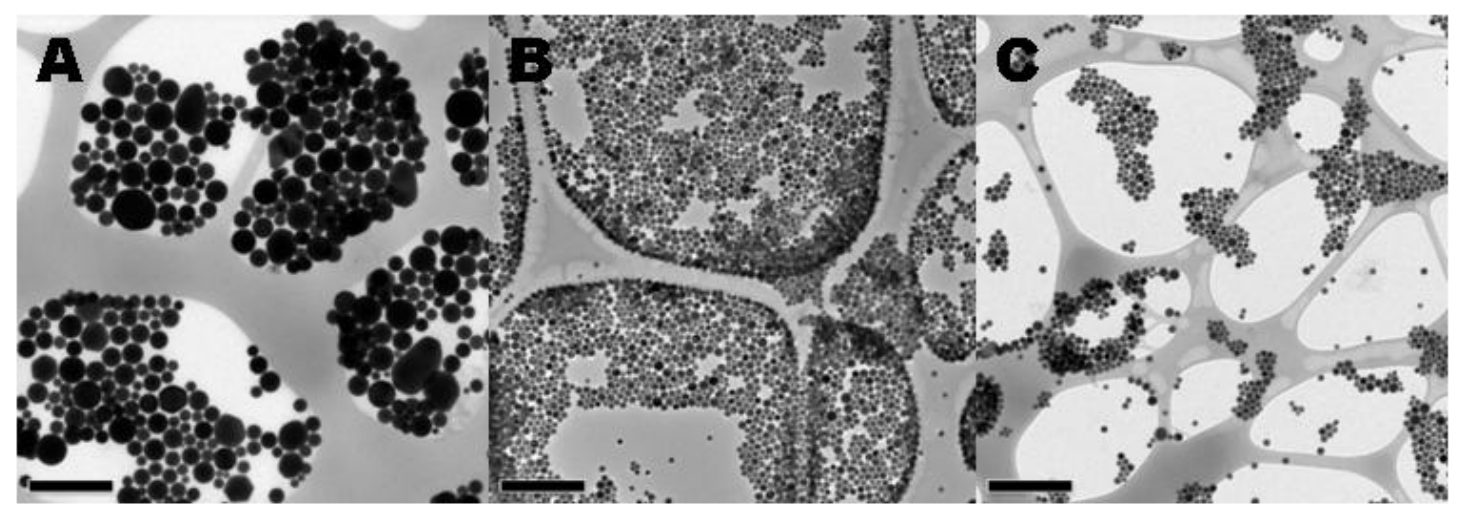

Figure 4.1. Bismuth particles synthesized by hot injection of iodobismuth precursor into neat hexadecylamine $\quad$ (A) $1: 1 \quad$ (B) and $1: 3 \quad$ (C) hexadecylamine:hexadecane. All scale bars represent $500 \mathrm{~nm}$.

$\mathrm{Bil}_{3}$ was experimentally chosen as a bismuth source because of its ability to dissolve and form optically clear suspended solutions in primary amine 
solvents. While the bismuth source was not characterized, a visible color shift from the solid black $\mathrm{Bil}_{3}$ to a bright orange solution was observed, consistent with the formation of soluble iodobismuth clusters. $\mathrm{Bi}_{3}$ was stirred in hexadecylamine (2.5 mg/mL or $5 \mathrm{mg} / \mathrm{mL}$ ) at $100{ }^{\circ} \mathrm{C}$ for over an hour prior to particle synthesis to encourage equilibrium and homogeneity of the bismuth source. This solution was then added to a hot $\left(200^{\circ} \mathrm{C}\right)$ solution of hexadecylamine or hexadecylamine and hexadecane. The rapid onset of bismuth reduction, which, coupled with a dilution of the bismuth source, encouraged the growth and stabilization of small, relatively uniform, elemental bismuth particles. When particles were grown in only hexadecylamine, larger and more irregular morphologies were observed (Figure 4.1 A) Particles grown in mixtures of hexadecane and hexadecylamine show smaller and more uniform size distributions, regardless of the ratio of solvents (Figure 4. $1 \mathrm{~B}$ and C.) This phenomenon can be explained by the higher degree of solvent organization that should take place in solution and on the surface of the growing particles when hexadecane is present in solution. In this case, a continuous phase of hydrophobic solvent should force hexadecylamine to form reverse micelles around developing particles. This would isolate particles from growth, ripening, or fusion and would explain the higher particle homogeneity observed. Particles synthesized in neat hexadecylamine appear larger and more irregular, which suggests a higher rate of ripening or fusion during synthesis in the absence of a hydrophobic barrier. 

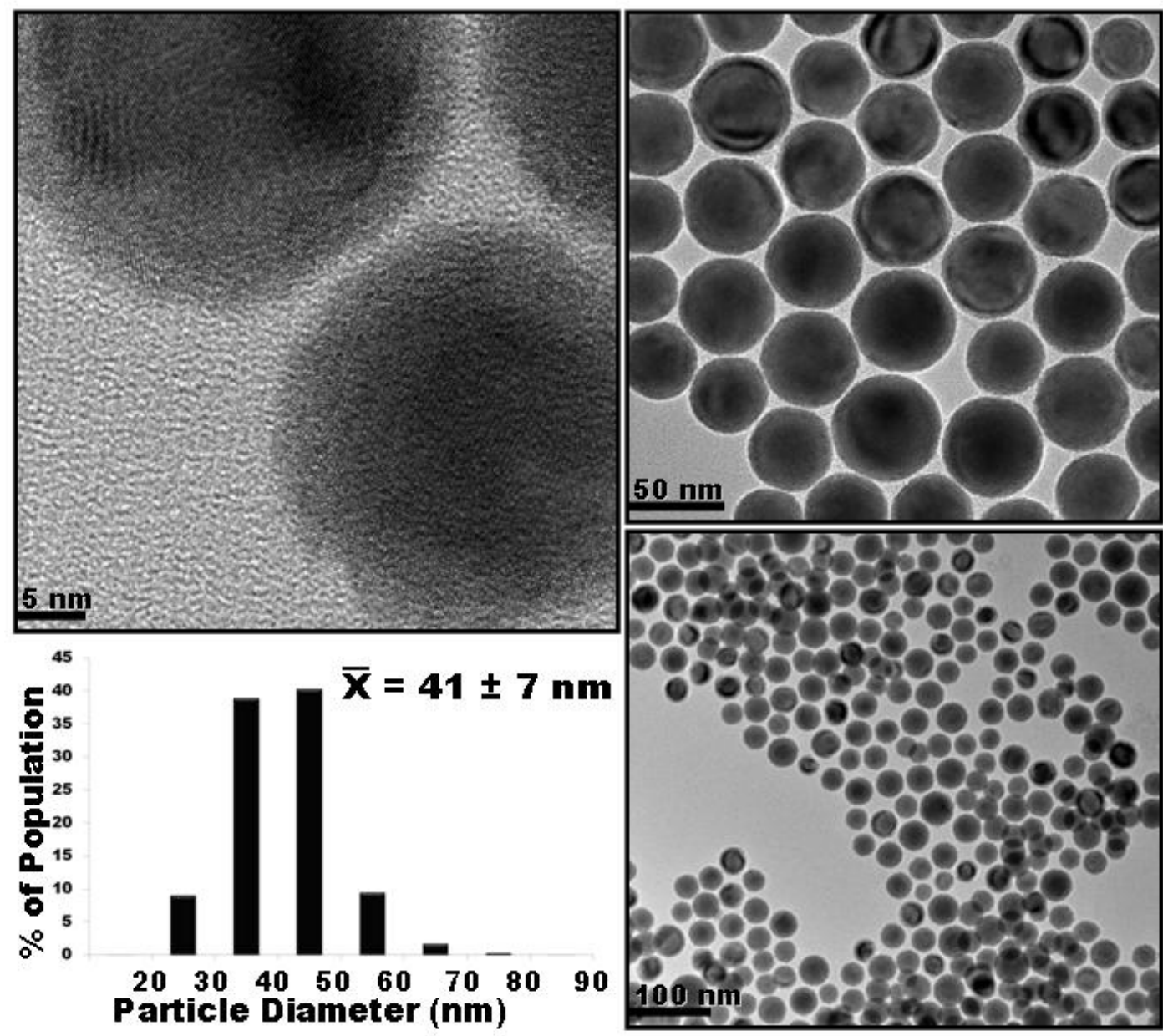

Figure 4.2. Bismuth particles synthesized in a 1:1 hexadecane:hexadecylamine mixture. Particles show lattice fringes with an average $d$-spacing of $3.2 \AA$, consistent with the (012) plane of rhombohedral bismuth metal (top left) and a relatively spherical morphology with an average diameter of $41 \pm 7 \mathrm{~nm}(\mathrm{n}=707)$.

Transmission Electron Microscopy (TEM) studies revealed spherical bismuth nanoparticles $41 \pm 7 \mathrm{~nm}$ in diameter (Figure 4.2). Lattice fringes of $3.3 \AA$, which indicated the theoretical (012) $d$-spacing of the rhombohedral (R-3m) phase of crystalline bismuth, were observed in several particles at high 
magnification. Particles show some long-range organization and packing, especially by SEM (Figure 4.3). When colloid solutions were measured by Dynamic Light Scattering (DLS), particles had large and irregular ( $>1 \mathrm{um}$ ) hydrodynamic sizes which suggests that particles in solution self-organize or flocculate.

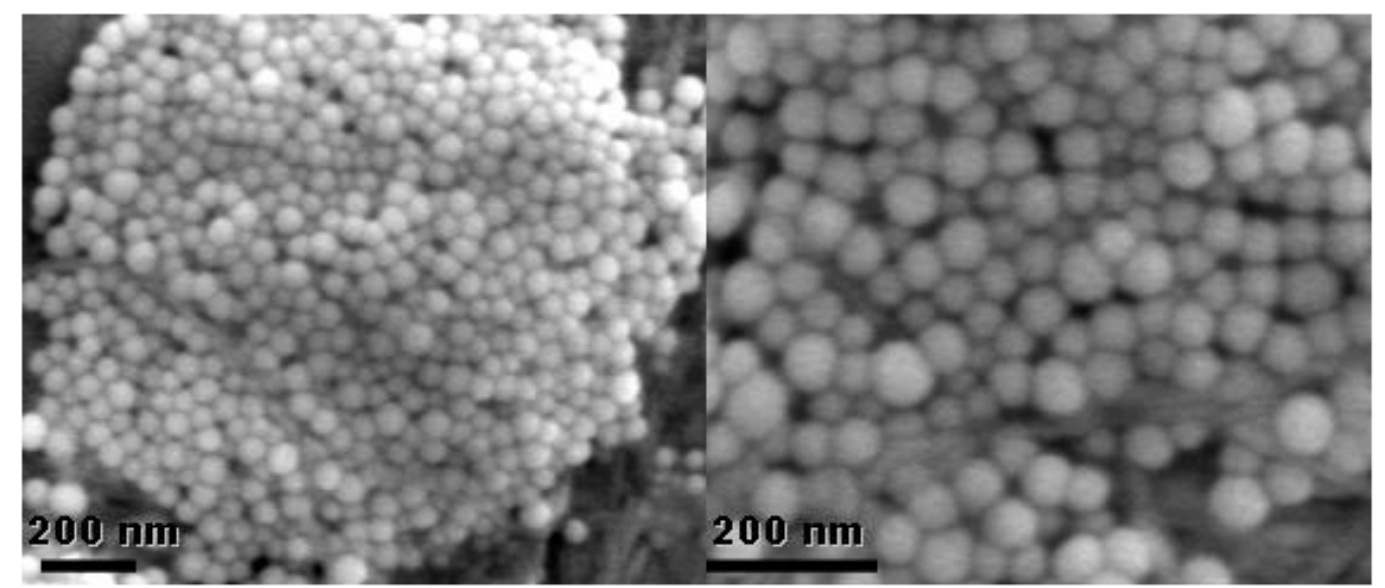

Figure 4.3. SEM images of bismuth particles synthesized in hexadecylamine and hexadecane. Particles are highly aggregated and show some degree of self-organization.

Because the particles were synthesized at very high temperatures, above the expected melting temperature of bismuth nanoparticles(20-22), the cooling rate and environment after synthesis should affect the morphology of the final nanocrystal. Allowing solutions to sit at synthetic temperatures in the absence of vigorous stirring, or cooling the particles at relatively slow rates, particularly highly concentrated synthetic solutions, enabled the growth of highly faceted and irregular nanocrystals. Figure 4.4 shows representative images of 
particle morphologies obtained when cooling rates were relatively slow. To form the homogeneous and rounded nanocrystals, shown in Figure 4.2, after a 5 minute synthesis at $200{ }^{\circ} \mathrm{C}$, particles were rapidly cooled by diluting the synthetic solution in iced toluene.
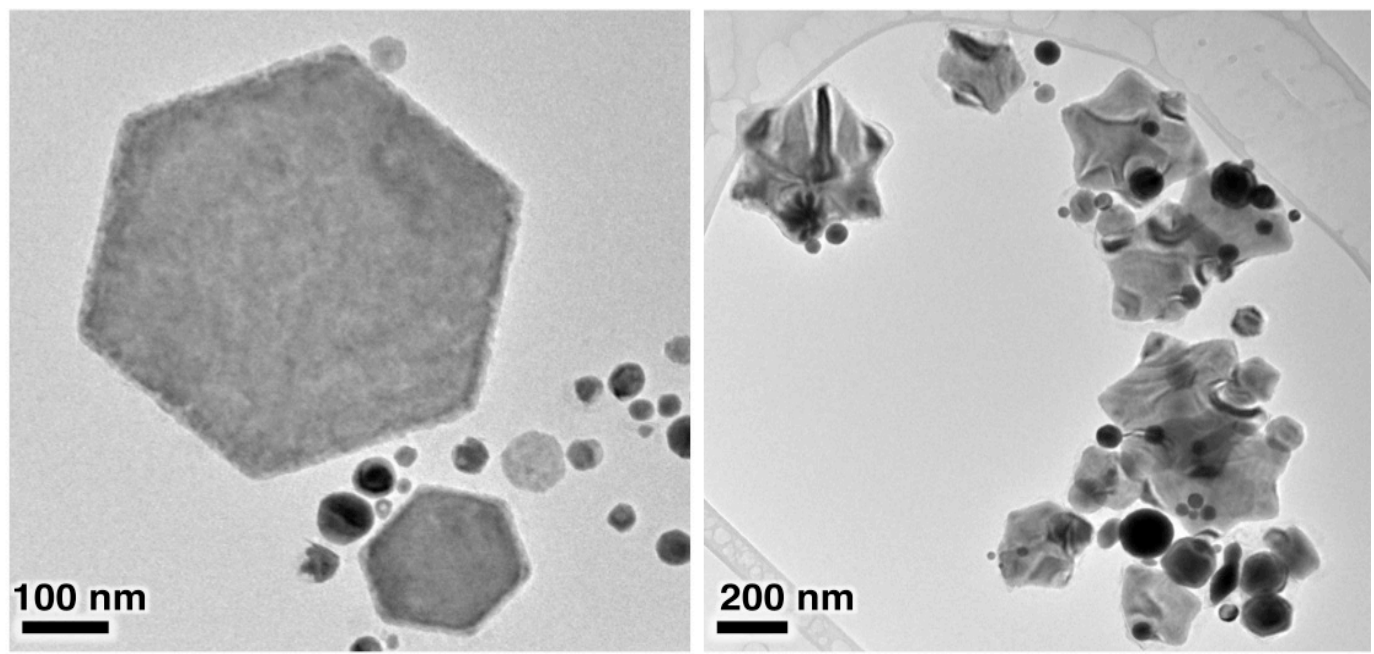

Figure 4.4. Representative morphologies of bismuth nanocrystals obtained when synthetic solutions at reaction temperature were not rapidly cooled. Hexagonal shapes (left), with $120^{\circ}$ facet angles were the most commonly observed morphologies. Hexagonal nanostars (right) are a novel bismuth nanocrystal morphology.

FT-IR of particles suggests the organic particle surface stabilizer is primarily hexadecylamine. The major FT-IR absorbance signature for hexadecylamine is evident on the bismuth nanoparticle surface. A pair of absorbance peaks around $3300 \mathrm{~cm}^{-1}$ from symmetric and asymmetric $\mathrm{v}_{\mathrm{N}-\mathrm{H}}$ stretches of the terminal $-\mathrm{NH}_{2}$ group on the ligand would indicate either a dative 
$\mathrm{R}-(\mathrm{H})_{2} \mathrm{~N}: \rightarrow \mathrm{Bi}$ coordination interaction or a $\mathrm{R}-(\mathrm{H}) \mathrm{N}-\mathrm{Bi}$ (amidometal) bond. Neither stretch is indistinguishable from the background, and further characterization will be necessary to elucidate the binding mode. However the presence of hexadecylamine on the surface of the particles, which have been extensively washed, suggests a chemical bond between the amine and the bismuth particle.

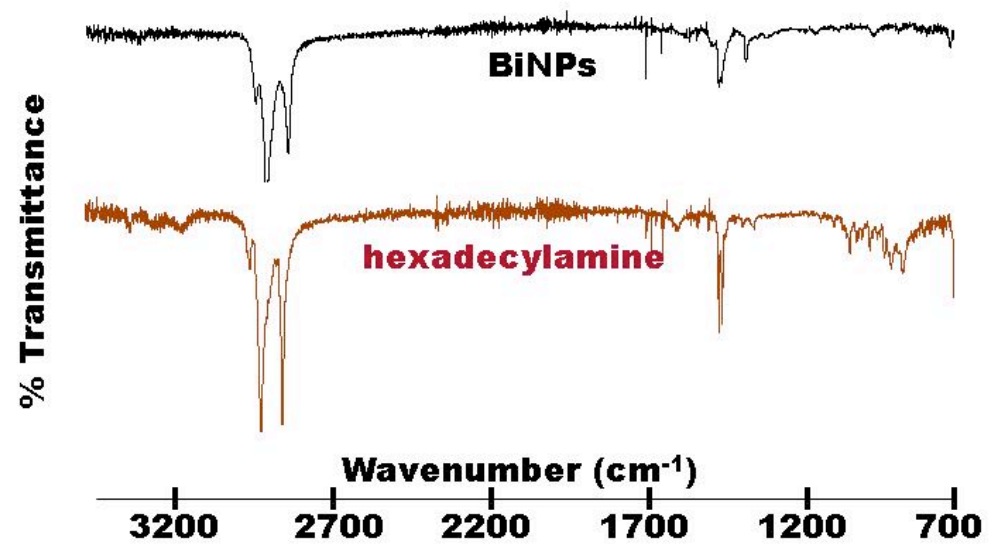

Figure 4.5. FT-IR spectrum of dried bismuth nanoparticles (top, black), and of neat hexadecylamine (bottom, red).

Thus while the nature of the amine-bismuth interaction is not elucidated by the FT-IR profile, it can be concluded that there is a stabilizing chemical or physical interaction by the primary amine on the bismuth surface.

\subsection{Conclusion}

We report the synthesis of $41 \pm 7 \mathrm{~nm}$ bismuth nanoparticles from $\mathrm{Bi}_{3}$ in a hexadecylamine and hexadecane solution at $200{ }^{\circ} \mathrm{C}$. Addition of hexadecane to 
the reaction solution yields smaller and more homogenous particles, and a rapid cooling of particles is necessary to yield rounded uniform particles. Particles show some degree of self-organization when imaged by SEM, and have hexadecylamine on the surface, as characterized by FT-IR. We anticipate these particles to be useful in experimental and practical applications where synthesis of bismuth nanoparticles using expensive reagents or laboratory equipment may be cost prohibitive.

\section{References:}

(1) Boukai, A.; Xu, K.; Heath, J. R. Size-Dependent Transport and Thermoelectric Properties of Individual Polycrystalline Bismuth Nanowires. Advanced Materials 2006, 18, 864-869.

(2) Carotenuto, G.; Hison, C. L.; Capezzuto, F.; Palomba, M.; Perlo, P.; Conte, P. Synthesis and thermoelectric characterisation of bismuth nanoparticles. Journal of Nanoparticle Research 2008, 11, 1729-1738.

(3) Hostler, S. R.; Qu, Y. Q.; Demko, M. T.; Abramson, A. R.; Qiu, X.; Burda, C. Thermoelectric properties of pressed bismuth nanoparticles. Superlattices and Microstructures 2008, 43, 195-207.

(4) Son, J. S.; Park, K.; Han, M.; Kang, C.; Park, S.; Kim, J.; Kim, W.; Kim, S.; Hyeon, T. Large-Scale Synthesis and Characterization of the Size-Dependent Thermoelectric Properties of Uniformly Sized Bismuth 
Nanocrystals. Angewandte Chemie International Edition 2011, 50, 13631366.

(5) Heremans, J.; Thrush, C. M. Thermoelectric power of bismuth nanowires. Phys. Rev. B 1999, 59, 12579.

(6) Rabin, O.; Manuel Perez, J.; Grimm, J.; Wojtkiewicz, G.; Weissleder, R. An X-ray computed tomography imaging agent based on long-circulating bismuth sulphide nanoparticles. Nature Materials 2006, 5, 118-122.

(7) Brown, A. L.; Goforth, A. M. pH-Dependent Synthesis and Stability of Aqueous, Elemental Bismuth Glyconanoparticle Colloids: Potentially Biocompatible X-ray Contrast Agents. Chemistry Materials 2012.

(8) Wang, F.; Tang, R.; Yu, H.; Gibbons, P. C.; Buhro, W. E. Size- and ShapeControlled Synthesis of Bismuth Nanoparticles. Chemistry of Materials 2008, 20, 3656-3662.

(9) Richards, V. N.; Shields, S. P.; Buhro, W. E. Nucleation Control in the Aggregative Growth of Bismuth Nanocrystals. Chemistry of Materials 2011, 23, 137-144.

(10) Yarema, M.; Kovalenko, M. V.; Hesser, G.; Talapin, D. V.; Heiss, W. Highly Monodisperse Bismuth Nanoparticles and Their Three-Dimensional Superlattices. Journal of the American Chemical Society 2010, 132, 15158-15159. 
(11) Briand, G. G.; Burford, N. Coordination complexes of bismuth(III) involving organic ligands with pnictogen or chalcogen donors. In Advances in Inorganic Chemistry; Academic Press, 2000; Vol. Volume 50, pp. 285-357.

(12) Hiramatsu, H.; Osterloh, F. E. A Simple Large-Scale Synthesis of Nearly Monodisperse Gold and Silver Nanoparticles with Adjustable Sizes and with Exchangeable Surfactants. Chemistry of Materials 2004, 16, 25092511.

(13) Lindsjö, M.; Fischer, A.; Kloo, L. Anionic Diversity in lodobismuthate Chemistry. Zeitschrift für anorganische und allgemeine Chemie 2005, 631, $1497-1501$.

(14) Advances in Inorganic Chemistry; Academic Press, 1994.

(15) Hofmann, P. The surfaces of bismuth: Structural and electronic properties. Progress in Surface Science 2006, 81, 191-245.

(16) Xu, Z.; Shen, C.; Hou, Y.; Gao, H.; Sun, S. Oleylamine as Both Reducing Agent and Stabilizer in a Facile Synthesis of Magnetite Nanoparticles. Chemistry of Materials 2009, 21, 1778-1780.

(17) Shevchenko, E. V.; Bodnarchuk, M. I.; Kovalenko, M. V.; Talapin, D. V.; Smith, R. K.; Aloni, S.; Heiss, W.; Alivisatos, A. P. Gold/Iron Oxide Core/Hollow-Shell Nanoparticles. Advanced Materials 2008, 20, 43234329. 
(18) Mazumder, V.; Sun, S. Oleylamine-Mediated Synthesis of Pd Nanoparticles for Catalytic Formic Acid Oxidation. Journal of the American Chemical Society 2009, 131, 4588-4589.

(19) Chen, M.; Feng, Y.-G.; Wang, X.; Li, T.-C.; Zhang, J.-Y.; Qian, D.-J. Silver Nanoparticles Capped by Oleylamine: Formation, Growth, and SelfOrganization. Langmuir 2007, 23, 5296-5304.

(20) Olson, E. A.; Efremov, M. Y.; Zhang, M.; Zhang, Z.; Allen, L. H. Sizedependent melting of Bi nanoparticles. Journal of Applied Physics 2005, 97, 034304-034304-9.

(21) Kharissova, O. V.; Kharisov, B. I. Nanostructurized Forms of Bismuth. Synthesis and Reactivity in Inorganic, Metal-Organic, and Nano-Metal Chemistry 2008, 38, 491-502.

(22) Wang, Y.; Xia, Y. Bottom-Up and Top-Down Approaches to the Synthesis of Monodispersed Spherical Colloids of Low Melting-Point Metals. Nano Letters 2004, 4, 2047-2050. 


\section{Chapter 5 - Highly X-ray Opaque Polymer/Bismuth Microparticle Composite Materials}

\section{Adapted from:}
A. Goforth, A. Brown
World Intellectual Patent Organization application
WO Patent 2,012,170,569, 2012

\subsection{Abstract}

The synthesis of bismuth microparticles and their incorporation into polymeric matrices for the development of highly X-ray opaque composite materials, is described. Particles were fabricated by ball milling bulk bismuth in a coordinating solvent, such as dihydroxy acetone, 1-pentene, and styrene, and then mixed with liquid polymers such as silicone, polyurethane, polystyrene, and latex to form composites. Composite materials are shown to be highly X-ray attenuating and can be molded to make X-ray opaque ribbons for surgical sponge markers, inks, and other items with specified physical properties.

\subsection{Introduction to X-ray Opaque Composite Materials}

X-ray photography is a ubiquitous technique in modern medicine with the ability to noninvasively image certain internal biological structures and to survey for foreign objects. In efforts to avoid surgical retained foreign objects (RFO) some medical tools and devices have been specifically impregnated or tagged 
with heavy atomic number elements so that they can be easily located inside a patient by X-ray imaging. Surgical sponge retentions account for greater than half of all RFOs because of their small size, frequent use and the difficulty in visually detecting soiled sponges against a morphologically similar anatomical background.(1) Surgical items that frequently become RFOs, such as sponges and other items composed of low atomic number elements, are the most commonly tagged or modified for X-ray imaging. The addition of an X-ray opaque $\mathrm{BaSO}_{4}$ based composite material molded as a thread or ribbon and sewn into a surgical sponge was developed decades ago and are commonly used in the US, but poor X-ray contrast makes them difficult for radiological detection, even under ideal imaging conditions.(2) More recently, Radio Frequency Identification (RFID) tagged sponges and operating room detectors have been introduced, but the high cost and required changes to operating room protocols have slowed embrace of RFID monitoring for RFO.(3) Unlike RFID scanning, X-ray imaging of patients prior to surgical wound closure is common practice; thus an inexpensive X-ray contrast marker with greater X-ray opacity than markers currently available would make retained surgical sponges easier to image and would be an optimal solution to RFO detection prior to surgical wound closure. The development of technologies for detection of surgical implements prior to closure of a surgical wound has been prompted by the high likelihood of further medical complications arising from RFOs, including local and systemic infections, and additional corrective surgical procedures that often result in lawsuits. 
While surgical sponges are the most likely to objects to become ROF, other medical devices or implements could benefit from a highly X-ray opaque contrast material additive. Embedding highly X-ray attenuating materials into items used in invasive treatments, or to track implant devices, would enable Xray imaging of materials which are normally X-ray transparent, such as plastics. Fluoroscopy, the technique whereby continuous X-ray images are collected to obtain dynamic imaging information of internal structures, would benefit from the incorporation of highly X-ray opaque material or tags by enabling low X-ray attenuating implements to be used and imaged by the physician during a procedure. This could include polymer based implants, catheters, stents, picc lines and equipment that may break or fragment during a procedure.(4) In a few specific applications highly radiopaque, yet mechanically flexible, medical implements could be useful. This could include replacements for the relatively expensive silver guide wires used in intra-arterial procedures, such as angioplasty.(5) Furthermore improved, more flexible, durable and thinner X-ray shielding materials could be generated from the materials described in this chapter. Most X-ray shielding materials are composed of lead salts because this element has a high atomic number and is extremely low cost. However, the inherent toxicity of lead inhibits its use in medical applications where a device may have intimate contact with a patient or medical personnel. Thus shielding devices used in a medical setting require protection barriers, such as heavyweight vinyl between the shielding material and environment. An X-ray 
attenuating material that does not have these toxicity concerns would not require the protection barriers.

Bismuth has an X-ray attenuation profile similar to lead, but the wellknown biological compatibility of this element eliminates a number of health concerns that arise from the use of lead. Because contact with lead is generally considered unsafe it cannot be used as an X-ray contrast marker for applications where routine or accidental contact may occur. Elements on the periodic table with large atomic numbers, and thus a high X-ray attenuation, are generally toxic, are prohibitively expensive, or both. Gold is a heavy, dense element and exploration of gold nanoparticles as intravenous X-ray contrast agents is an active area of research, but the price of gold (currently approx. $\$ 8,850$ per mol) is prohibitively expensive for this application. Platinum has a similar price per mole. Other dense elements, such as thallium, iridium and osmium are also prohibitively expensive, and are additionally extremely toxic.

To produce a material with the highest $\mathrm{X}$-ray attenuating potential in the smallest volume, the elemental form of the attenuating element will be required. Thus sheets of the elemental material will provide the highest X-ray attenuation per volume and weight. Because the medical applications described above will require a material which can be molded into a wide range of shapes and have a degree flexibility in the final product, microparticles of highly X-ray attenuating material embedded in a polymer matrix should impart both desired properties. Bismuth molecular species and nanoparticles embedded in a polymer matrix 
could produce materials with a high X-ray attenuation as well, but the increased surface area of the smaller atoms, clusters or nanopartucles would require larger amounts of polymeric material relative to the number of X-ray attenuating atoms. Therefore, bismuth microparticle composites should require less polymeric material per functional atom, which will reduce the overall weight and volume of the contrast or shielding material. The development of a thinner, lighter and low toxicity composite material will enable development of a wide range of novel or improved medical devices which are highly X-ray attenuating or shielding.

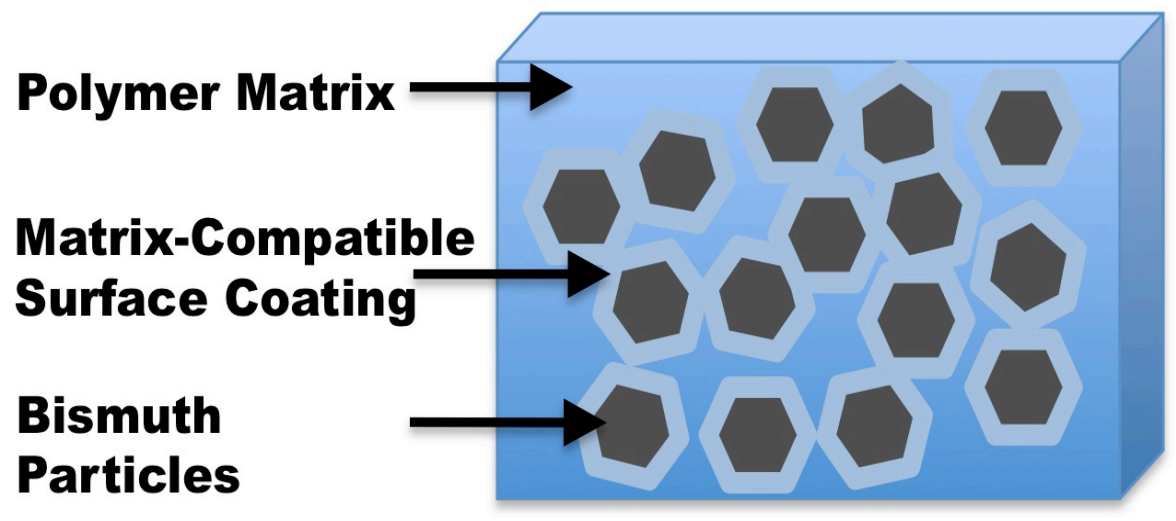

Scheme 5.1. Bismuth particle composite materials composed of a polymer matrix and bismuth particles that have been coated in an appropriate surfactant for matrix miscibility.

A bismuth microparticle additive for comoposite materials will need to be inexpensive to produce and should be miscible in a variety of solvents to enable incorporation in numerous types of polymeric matrices. The medical applications of a material possessing the properties of highly X-ray attenuating, moldable, flexible, non-toxic and inexpensive are numerous. In this research, material 
optimization and a performance assessment was focused on the development of a surgical sponge marker based on market evaluation and suggestions of a commercialization opportunity report done by the Winter 2011 Engineering and Technology Management New Venture Management class (ETM 562/662). In addition to the surgical sponge markers, other composite materials and devices and X-ray opaque inks discussed in this chapter were developed and incorporated in a patent application (U.S. Provisional Application No. 61/493,913, filed June 6,2011$)$. A shielding and attenuation evaluation of surgical sponge prototype materials was also performed, and used to calculate the theoretical thicknesses of a bismuth microparticle shielding device.

\subsection{Methods}

\section{Bismuth Particle Preparation}

Bismuth powders were prepared by ball milling bulk bismuth and a solvent with or without an additional surfactant in a stainless steel grinding vial with tungsten carbide balls for 20 minutes. In a typical preparation $5 \mathrm{~g}$ of elemental bismuth (Rotometals, Bismuth Ingot 99.99\% pure) was milled with $5 \mathrm{~g}$ solid surfactant and in certain cases $10 \mathrm{~mL}$ of an appropriate solvent (such as 1,2 propane diol or 1-pentene). To prepare hydrophobic particles, $10 \mathrm{~mL}$ of 1 pentene (Acros Organics, 97\%) was used, with no additional surfactant added. For the synthesis of hydrophilic particles, $10 \mathrm{~mL}$ of 1,2-propane diol (Acros Organics 99\%, Extra Pure) and $5 \mathrm{~g}$ dihydroxyacetone (MP Biomedicals) was 
used. Styrene capped particles were milled in $10 \mathrm{~mL}$ styrene (Acros, 99\% stabilized). The particles, suspended in their milling solvents, were either sieved (Fisher Scientific Test Sieve no. 325, $45 \mu \mathrm{m}$ ) and washed with an appropriate solvent and collected by centrifugation or washed, collected, dried, then ground to a powder and sieved.

\section{Matrix Incorporation}

Particles were incorporated into a variety of polymer matrices, including silicone (GE 100\% silicone), polyurethane (VytaFlex 20 urethane), Latex (nheptane, natural rubber latex, Ross acid-free rubber cement) polystyrene (commercial packing material) and dextran (Fisher, $86 \mathrm{kDA}$ ) composite materials. In a typical preparation, the dried bismuth powder was weighed, the liquid matrix was added and the weight of both matrix and powder were recorded. If the liquid weight of the matrix changed upon drying (as in the case of silicone) this was considered and incorporated into the calculation. The liquid unset polymers and the bismuth microparticles were mixed, in some cases with the addition of a carrier solvent such as pentane for silicone, or in the case of polystyrene, toluene (ChemProducts), and water in the case of dextran. No carrier solvent was used in preparation of polyurethane composites. The composites were either allowed to air-dry, or, in the case of the polyurethane composites, were placed under vacuum for 5 minutes to remove air bubbles before pressing into a PTFE mold. 
The final composites contained the homogenously distributed bismuth micro particles as a known weight percent of the dried material.

\section{SEM Imaging}

SEM imaging of particles and composites was done on a FEI Sirion XL30 FEG SEM. Powder samples were mounted on SEM stubs with adhesive, or composite materials were thinly sliced with a razor and mounted on adhesive. All samples were sputter coated with gold (PELCO 91000 Sputter Coater) for 60 sec.

\subsection{Results and Discussion}

Production of large quantities of inexpensive micronparticles was undertaken using top down protocol. In this process, a chunk of elemental bismuth is ball milled in an excess of coordinating surfactant to produce a wide range of particle sizes. The resultant particle mixture is washed in an appropriate solvent and collected by centrifugation. Particles are size selected with mesh sieves or syringe filters. These particles generally retain the miscibility of the coordinating solvent, thus enabling synthesis of particles which can be surface tailored to be dispersed in a wide range of matrices. Coordinating solvents were compared for surface sorption on milled bismuth and solvent choice was assessed for morphology effects on particles. Hydrophobic and hydrophilic particles were synthesized. These particles were embedded in different matrix 
polymers (such as silicone, polyurethane, latex and styrene) for demonstration of application and X-ray opacity studies.

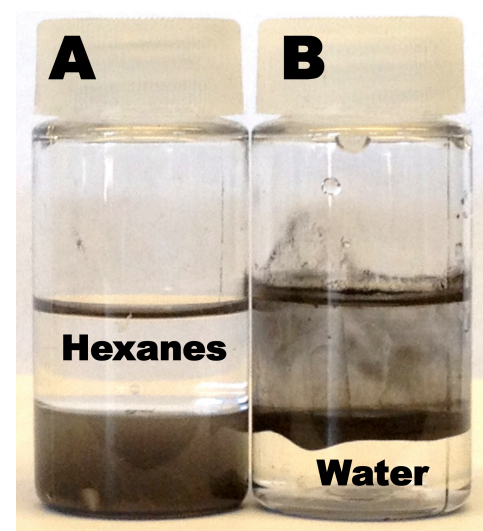

Figure 5.1. Bimsuth particle miscibility illustrated by solvent separation. Particles milled in dihydroxy acetone $(A)$ or in 1-pentene $(B)$, show solubility preference for water or hexanes respectively.

\section{Aqueous Particles}

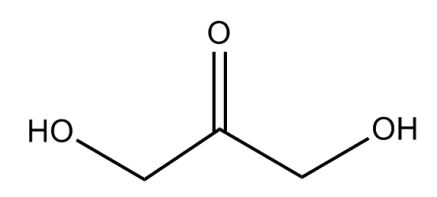

Scheme 5.2. Structure of dihydroxy acetone

Synthesis of particles soluble in water was undertaken by utilizing knowledge of suitable surfactants from previous nanoparticle synthesis experiments. As carbonyls had previously shown highly stabilizing to bismuth particle surfaces, dihydroxyacetone (DHA, Scheme 2) was milled with bismuth to produce particles with alcohol surface functionalities. These particles proved hydrophilic and were washed multiple times in water to remove excess surfactants and collected by 
centrifugation. (Figure 5.2) Particles were then dried at $140{ }^{\circ} \mathrm{C}$ in a ceramic crucible for greater than 2 hours and ground with a pestle. The dried powder was passed through a $45 \mu \mathrm{m}$ mesh sieve and stored in a glass jar at room temperature. Larger particles which did not pass through the sieve were recycled and processed as bulk bismuth.

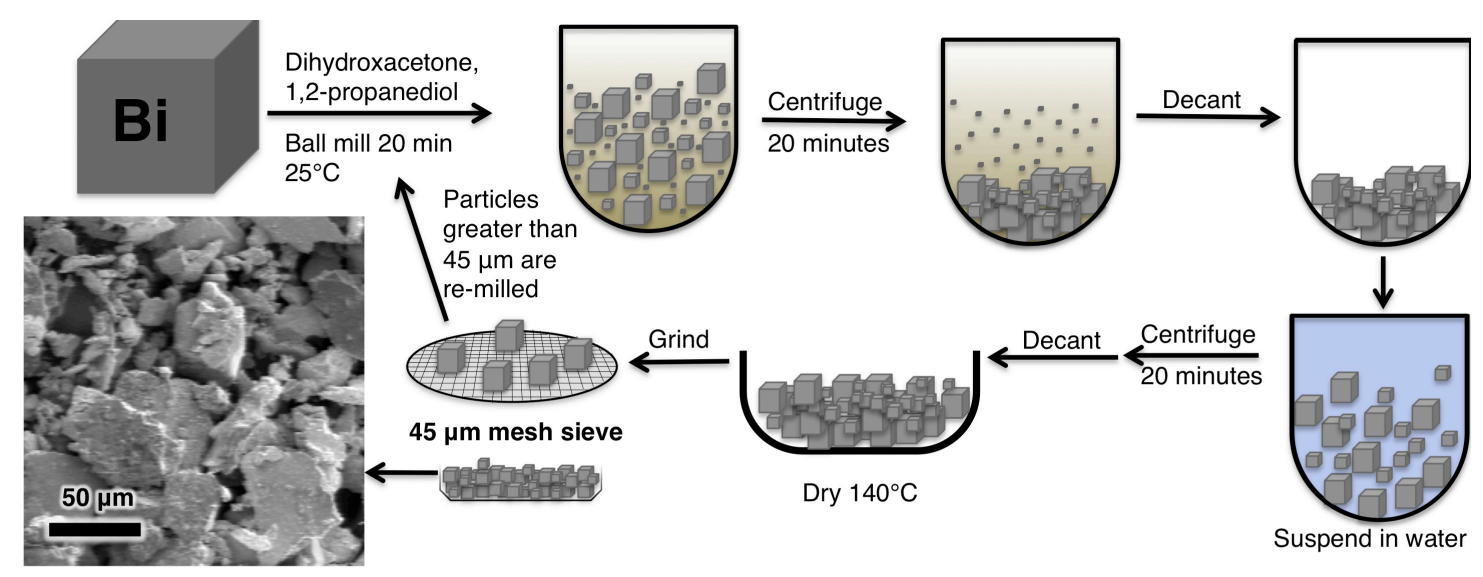

Figure 5.2. Illustration of microparticle synthesis, purification, size selection. SEM image insert shows dry sieved particles.

The primary application recommended by the Winter 2011 technology venture class was to develop a better radiopaque surgical sponge marker. The current commercially used makers are composed of a polymer, reported to be a composite material of polyvinylchloride, $60-83 \%$ barium sulfate and other plasticizers and colorants. (Tariff No.: 2833.27.0000) Sponge markers from a laparotomy sponge produced by Medline Industries were collected and used as a reference for market available radiopaque surgical sponge markers. A cross sections of these markers was measured by SEM and found to be approximately 
$400 \mu \mathrm{m}$. To create a marker with comparable dimensions, but with increased contrast additive weight ratios, polyurethane was used as the matrix polymer because of its high strength. Dry sieved DHA capped particles were measured by weight and an appropriate ratio of unpolymerized polyurethane solution was added. Bismuth particles and polymer solution were mixed and scooped onto a flat PTFE surface and placed in a vacuum desiccator to remove gas bubbles. Spacers of desired thickness (for $400 \mu \mathrm{m}$ thickness, 2 widths lab tape and 3 widths scotch tape) were placed proximal to particle mixture and a second piece of flat PTFE was placed on top of solution and clamped. Composite material was allowed to polymerize over night and heated $\left(125^{\circ} \mathrm{C}, 1\right.$ hour) after removal from mold. Shown in Figure 5. 3 is an SEM images of the synthesized particles (Figure $5.3 \mathrm{~A}$ ) and a schematic of their incorporation into a lquid polymeric material (Figure 5.3 B.) The resultant composite materials produced show visable particles encased in a polymer when imaged by SEM (Figure 5.3 C-F.) 

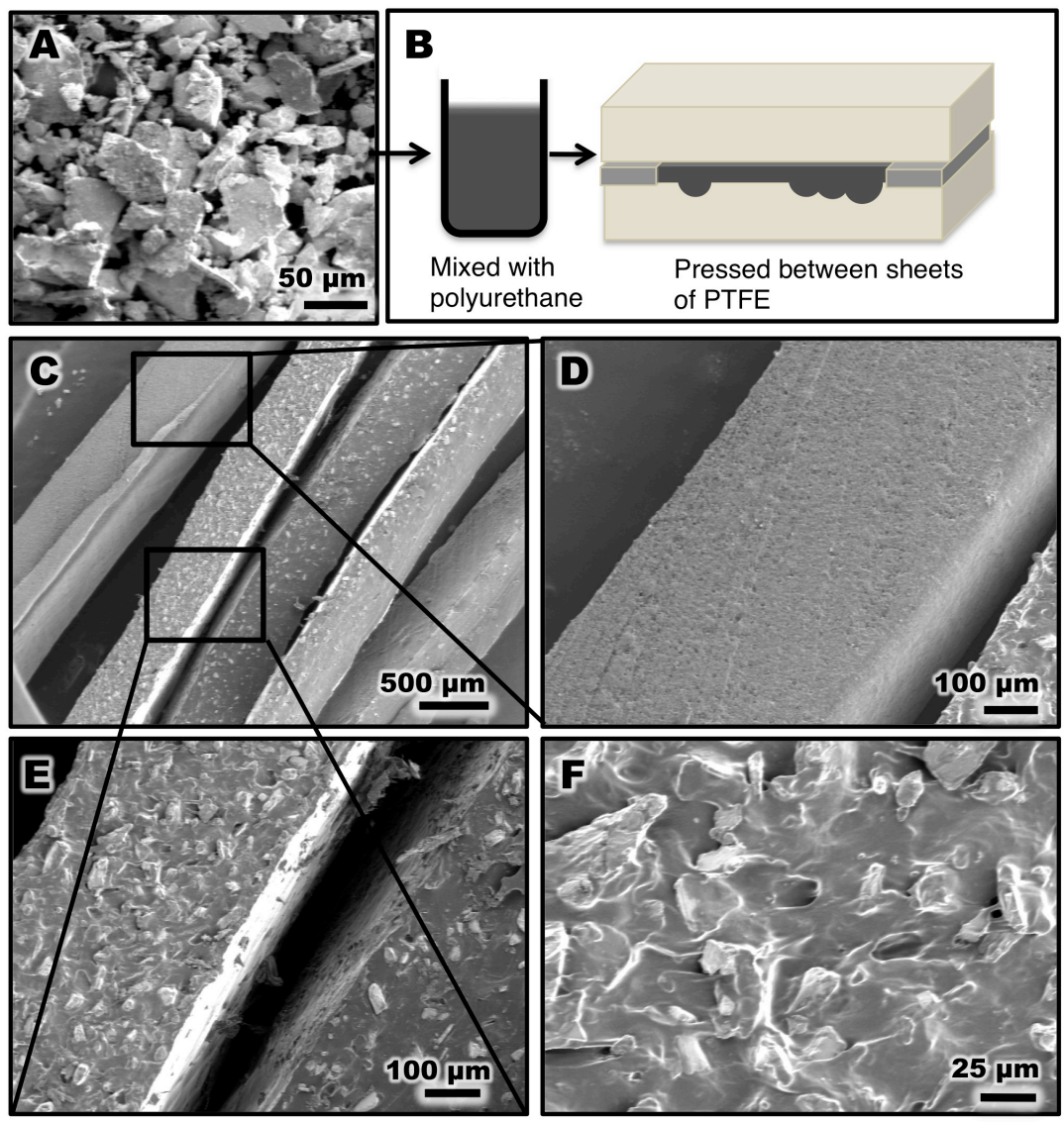

Figure 5.3. Polyurethane and commercially available X-ray opaque sponge markers. Dried DHA >45 $\mu \mathrm{m}$ sieved particles $(\mathrm{A})$ were mixed with liquid polyurethane and pressed between sheets of PTFE with spacers of a designated thickness (B). A Medline X-ray opaque sponge marker was imaged by SEM next to composites made of $85,60,40$, and $20 \%$ bismuth particles by weight (C, cross sections in order mentioned from upper left corner.) Marker composed of $85 \%$ by weight bismuth clearly shows particles embedded throughout polymer matrix $(E$ and $F)$. Medline marker shows a much smoother and more uniform appearance, presumably due to the smaller size of $\mathrm{BaSO}_{4}$ salt particles (D).

To compare attenuation of the commercially available surgical sponge markers (Medline) to the bismuth composite markers, X-ray attenuation was 
performed by Health Physics Northwest (HPNW) and is shown in Figure 5.4. The Medline markers show a similar attenuation profile to bismuth marker with $40 \%$ by weight loading. Interestingly, the attenuation for the $40 \%$ weight loaded bismuth marker dropped relative to the Medline marker at higher X-ray energies $(>100 \mathrm{keV})$ consistent with a theoretical drop in attenuation at energies above the k-edge of bismuth at $90 \mathrm{keV.(6)} \mathrm{Measurements} \mathrm{by} \mathrm{HPNW} \mathrm{included} \mathrm{thickness}$ analysis using a Peacock Dial Thickness Gauge, Model H and measured a 0.56, $0.55,0.51$ and $0.64 \mathrm{~mm}$ thickness for the Medline, 40, 60 and $85 \%$ by weight bismuth markers respectively. This was inconsistent with SEM measurements of the markers, which showed a thickness of approximately $400 \mu \mathrm{m}$. (Figure $5.3 \mathrm{C}$ ).

The $85 \%$ bismuth marker had an lead equivalent X-ray attenuation of 0.21 $\mathrm{mm}$. X-ray aprons used in medical facilities typically have a $0.5 \mathrm{~mm}$ lead equivalent attenuation(7), which would indicate a $1.2 \mathrm{~mm}$ thick sheet of the $85 \%$ bismuth composite material would provide similar radiation protection, if used as a shielding material. 


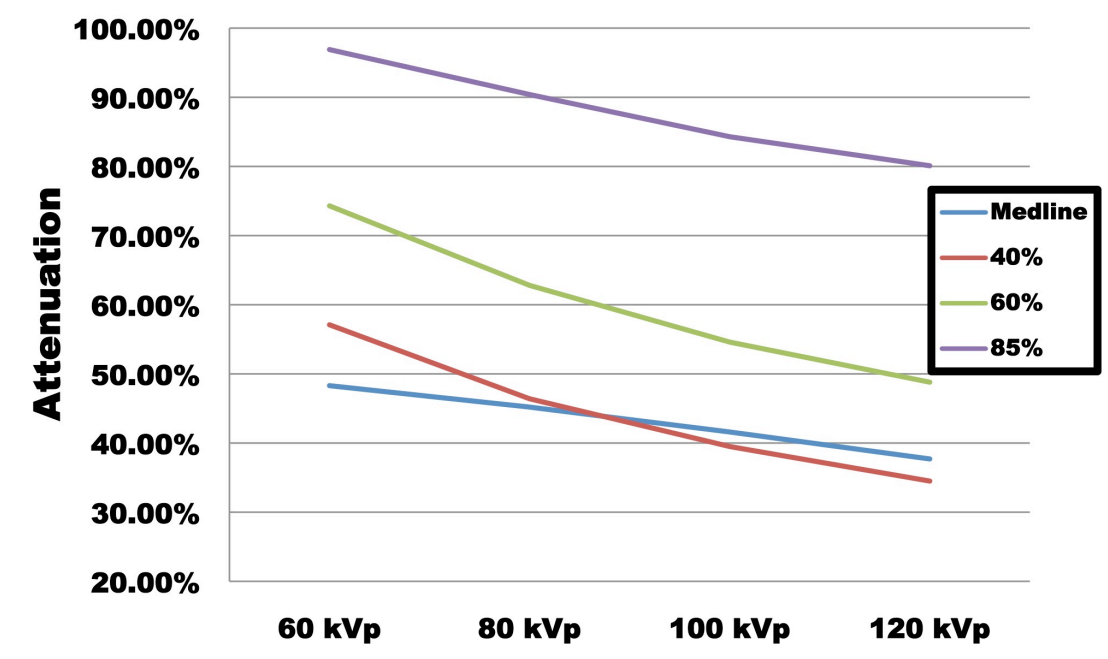

Figure 5.4. X-ray attenuation of loaded surgical sponge markers. Polyurethane composite materials were made with DHA capped bismuth particles $(<45 \mu \mathrm{m})$ embedded in a polyurethane matrix, and pressed to approximately $400 \mu \mathrm{m} .40,60$ and $85 \%$ bismuth particle composite materials by weight were compared to a standard Medline surgical sponge marker. The $85 \%$ by weight composite sample had an attenuation equivalent to $200 \mu \mathrm{m}$ of lead.

The $60 \%$ bismuth composite material would need to be $3.125 \mathrm{~mm}$. The primary advantage of these bismuth composite materials over a lead based shielding material would be no additional layer separating the patient from the attenuating material, commonly polyvinyl chloride in lead based shields. This would enable the manufacturing of lighter weight and substantially thinner personal radiation shielding equipment. These could be manufactured into a variety of protective products, such as gloves. 


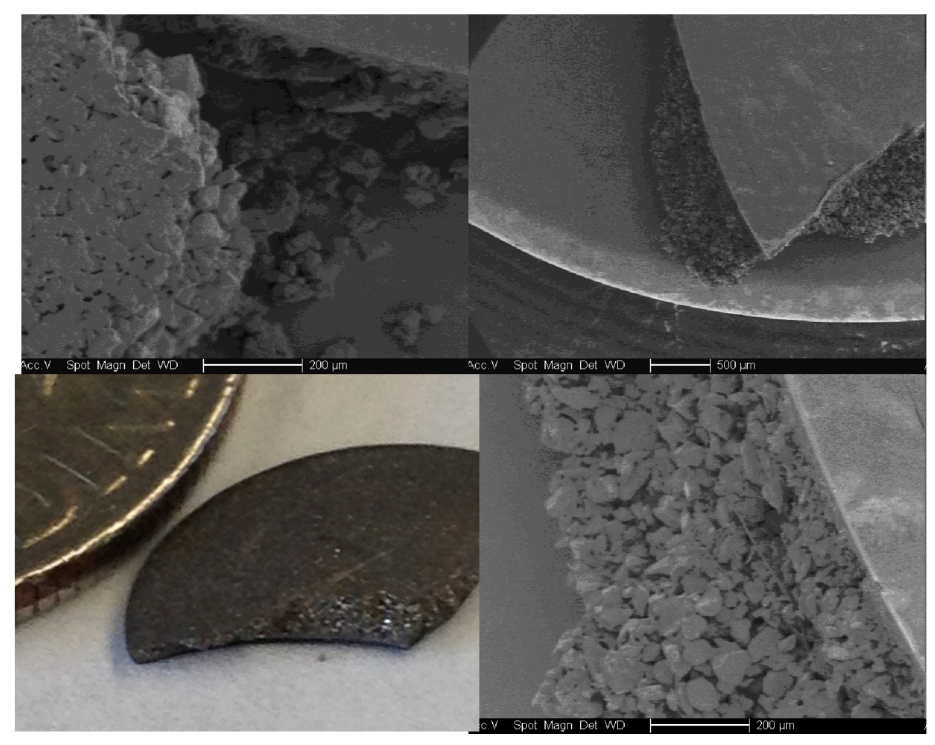

Figure 5.5. Hydrophilic particles embedded in a dextran polymer matrix and dried into a small disc. SEM images show aggregated particles held together at a high density, and smooth surface on top and bottom of the disc. Photograph of broken disc next to a dime is show for size comparison.

Other applications, and demonstrations of applications, of hydrophilic particles have included dextran polymer matrices particle liquids, which are flowable and water soluble and dry to form hard "discs" of bismuth particles illustrated in Figure 5.5. This method was also used to form water-soluble inks, which are then highly radiopaque, and can be easily re-dissolved in water for removal and recycled. As latex is common material used in a number of medical applications, latex suspensions of aqueous phase particles were made and dried into rubber sheets. (Figure 5.6) The latex composite materials were somewhat 
elastic, however they did show substantially decreased structural integrity over pure latex, possibly due to poor miscibility between the matrix and the particles.

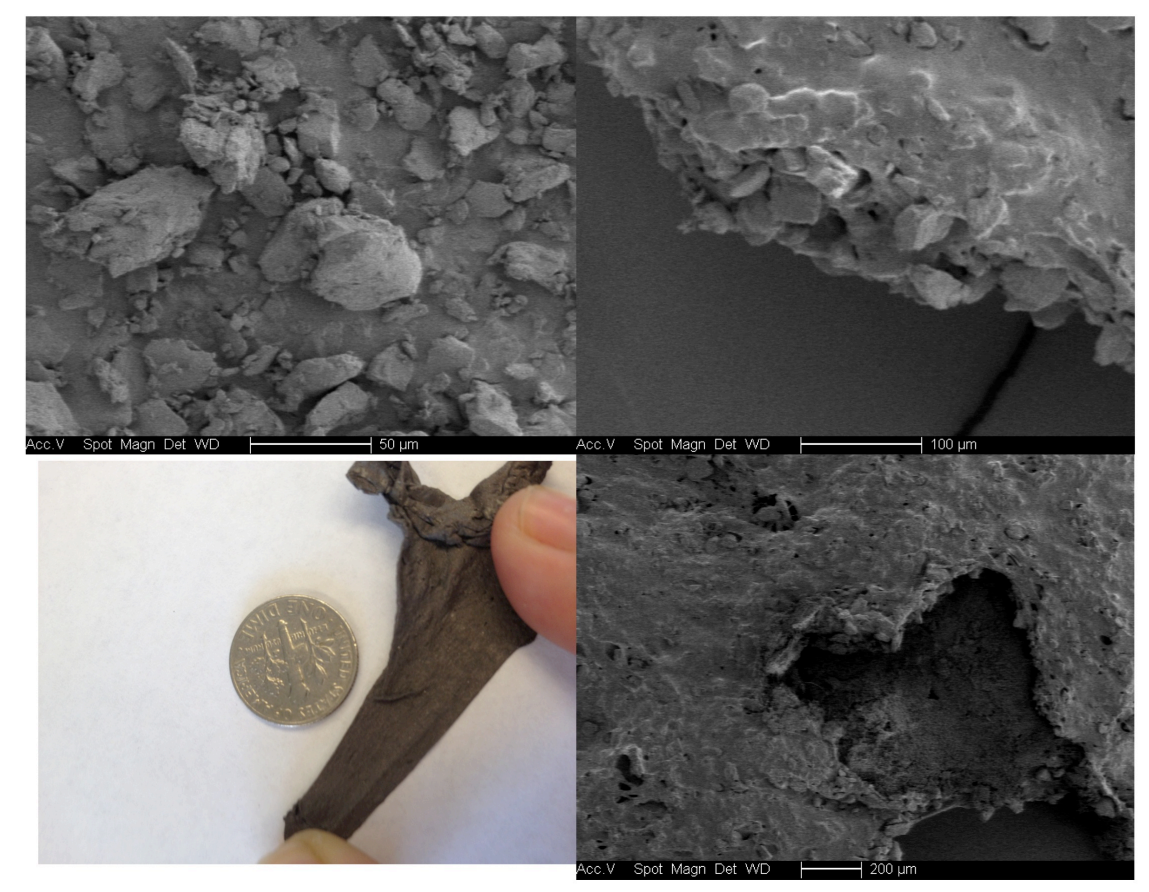

Figure 5.6. Latex composite materials. DHA capped particles were incorporated into a latex matrix.

\section{Hydrophobic Particles}

A number of plastics and rubbers used in medical items are formed using precursors which are soluble in organic solvents and thus a particle miscible in organic solvents was synthesized. 1-pentene was chosen as a surface sorbent due to high volatility and electron density at one end of the molecule. Comparison of surface morphologies of particles synthesized in 1-pentene and pentane 
suggest the double bond served as a source of electron density to surface stabilize particles during milling and prevent re-compacting of smaller particles into larger, polycrystalline particles. (Figure 5.7)

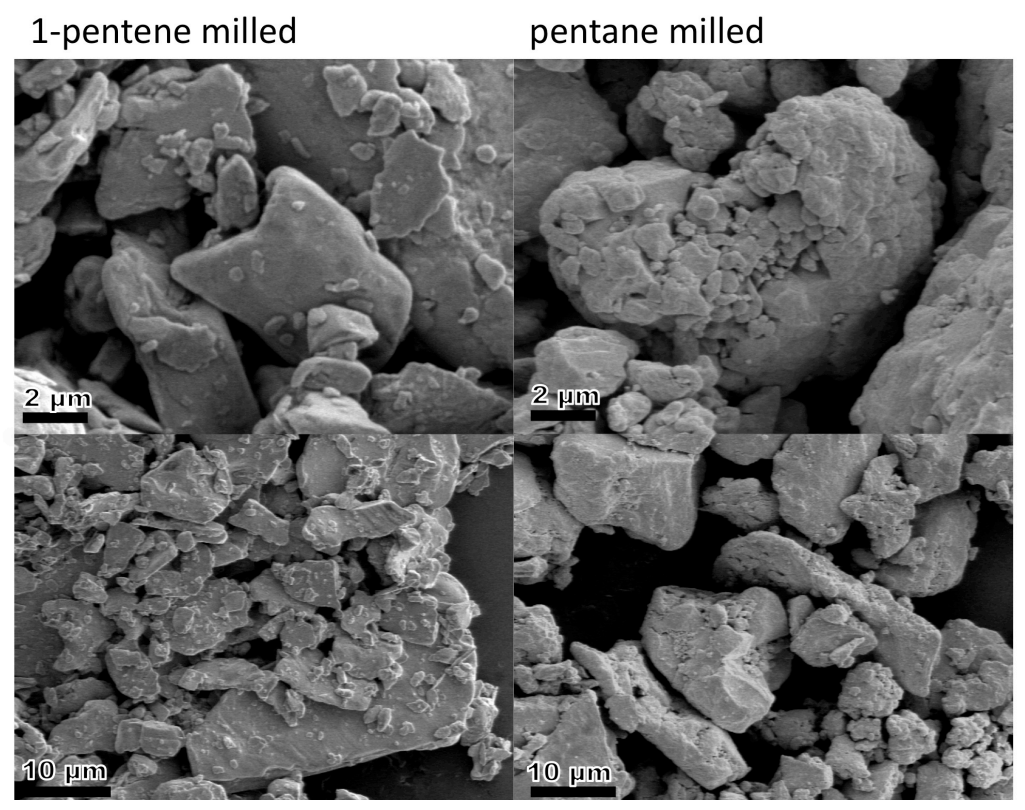

Figure 5.7. SEM images of bismuth micron particles made by ball milling bulk bismuth in 1-pentene (left) and pentane (right). Particle synthesized in non-surface adsorbing pentane appear to recombine and pack into aggregate particles, possibly due to instability of the freshly exposed bismuth surface. 


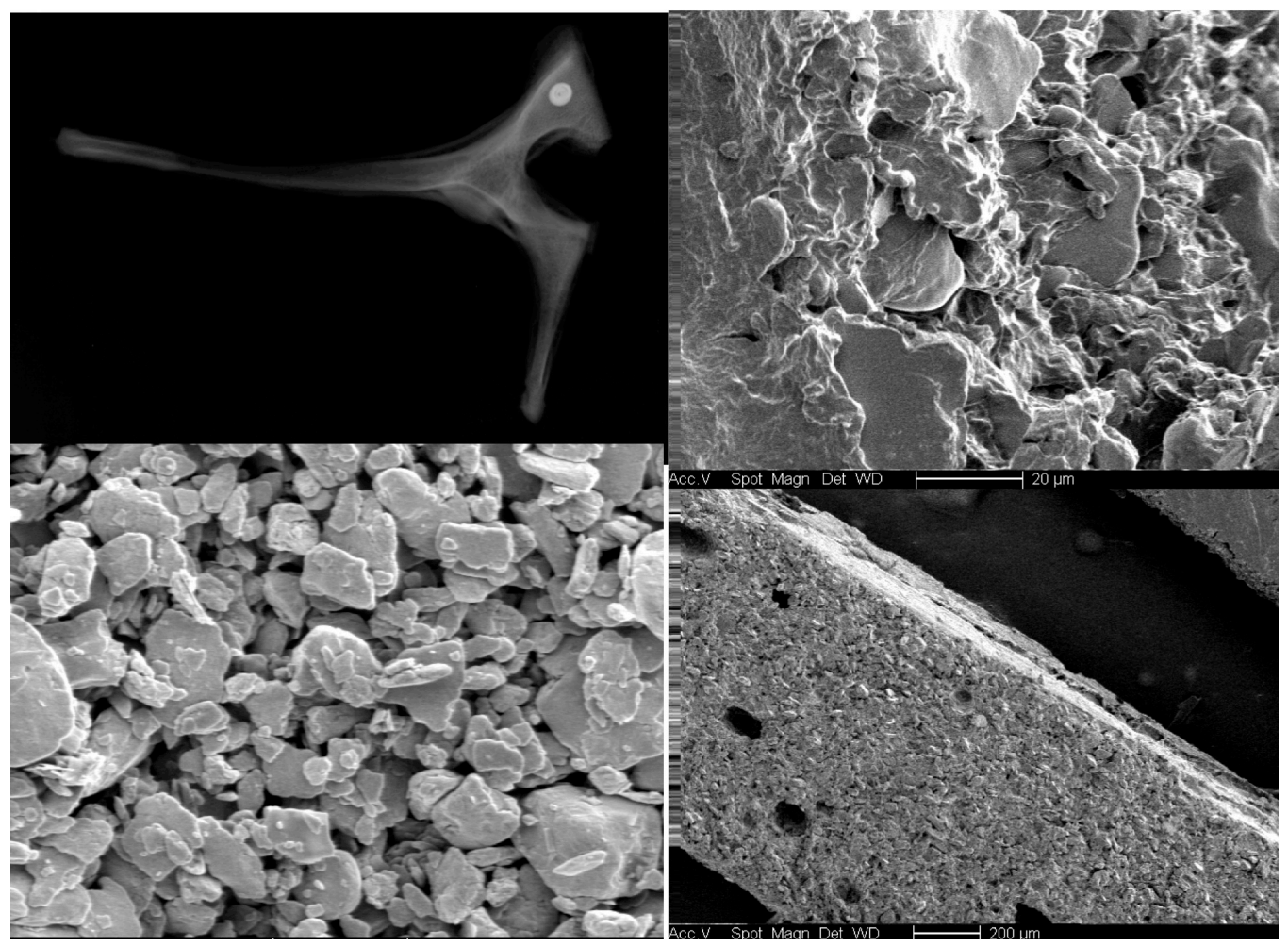

Figure 5.8. Silicone and hydrophobic bismuth particle composite materials. A silicone composite marker shows substantially enhanced attenuation over a bovine T-bone by X-ray (upper left). Particles were synthesized with 1pentene (lower left) and embedded in a silicone matrix (right top and bottom).

Incorporation of hydrophobic particles into silicone matrixes was used as initial illustrative proof of principal for surgical sponge markers as industrial grade silicone is inexpensive and easily molded and polymerized. Experiments using hydrophobic particles embedded in silicone were successful in that number of highly attenuating markers were synthesized, but difficulties with decreased marker attenuation arising from the formation of bubbles in the silicone, in conjunction with a highly reduced structural integrity of the silicone composite 
materials, lead us to abandon further development of silicone based sponge markers in favor of polyurethane based markers.

\section{Styrene based particle inks}

Styrene capped particles were synthesized and used to make polystyrene composite materials and polystyrene inks. For this application, styrene capped particles were incorporated into a solution of liquid polystyrene solvated in toluene. These inks could be stored in an air tight pasteur pipette pen and used to write on a variety of surfaces, or dried into brittle shapes. Illustrative use of these inks is shown in Figure 5.9, where particles in polystyrene (left) were used to trace the PSU logo (top right) and an X-ray image of the ink on top of a phantom of a hand shows high X-ray attenuation (lower right.) 


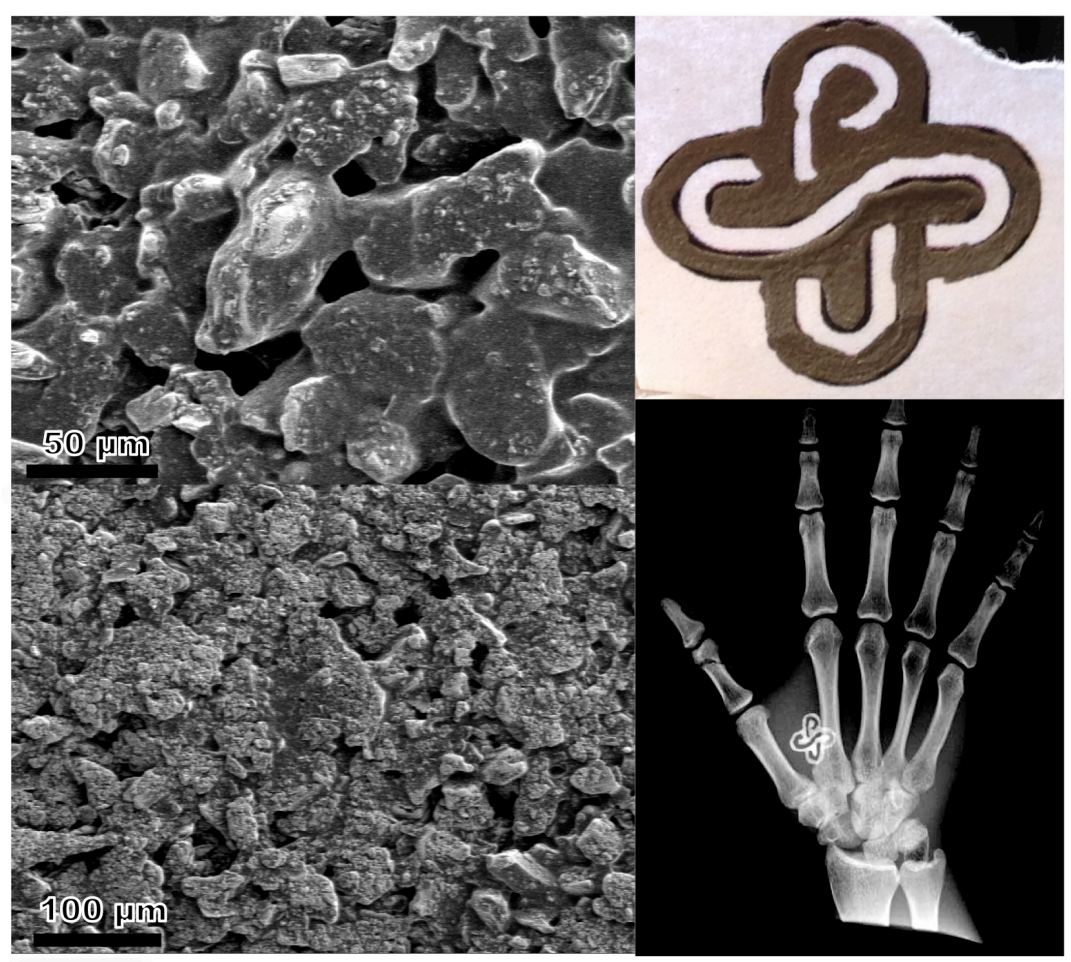

Figure 5.9. Illustrative use of styrene inks. Styrene milled particles with polystyrene and carried in toluene used as an ink. SEM images of particle ink (left), light photograph of ink (top right) and X-ray image of ink with a hand phantom (bottom right).

\subsection{Conclusions}

We demonstrate the use of inexpensively synthesized bismuth microparticles embedded in polymers as highly X-ray opaque contrast materials. The synthesis of these microparticles by ball-milling bulk bismuth in a coordinating solvent is a novel, but highly versatile method for producing surface 
functionalized bismuth particles. Without a surface coordinating solvent these particle recombine into polycrystalline aggregates. Hydrophobic, hydrophilic and cyclo-aromatic surface functionalized particles were synthesized and embedded in a variety of polymer matrixes for application illustrations.

\section{References:}

(1) Lincourt, A. E.; Harrell, A.; Cristiano, J.; Sechrist, C.; Kercher, K.; Heniford, B. T. Retained Foreign Bodies After Surgery. Journal of Surgical Research 2007, 138, 170-174.

(2) Kanne, J. P.; Phillips, G. S. Anterior mediastinal gossypiboma. Radiology Case Reports 2011, 6.

(3) When Surgeons Leave Objects Behind.

http://well.blogs.nytimes.com/2012/09/24/when-surgeons-leave-objectsbehind/ (accessed September 25, 2012).

(4) Misc Small Items. http://nothingleftbehind.org/Misc_items.html (accessed January 3,2013$)$.

(5) Palti-Wasserman, D.; Brukstein, A. M.; Beyar, R. P. Identifying and tracking a guide wire in the coronary arteries during angioplasty from X-ray images. IEEE Transactions on Biomedical Engineering 1997, 44, 152 -164.

(6) Materna, T.; Jolie, J.; Mondelaers, W.; Masschaele, B. Near K-edge measurement of the X-ray attenuation coefficient of heavy elements using a 
tuneable X-ray source based on an electron LINAC. Radiation Physics and Chemistry 2000, 59, 449-457.

(7) Allisy-Roberts, P. J.; Williams, J. R. Farr's Physics for Medical Imaging; Elsevier Health Sciences, 2007. 


\section{Terminal References}

\section{References from Chapter 1:}

(1) The new light: discovery and introduction of the X-ray. American Journal of Roentgenology 1995, 165, 1041-1045.

(2) Brenner DJ, H. H. Radiation exposure from medical imaging: Time to regulate? Journal of the American Medical Association 2010,304, 208-209.

(3) http://medical-dictionary.thefreedictionary.com/barium+enema (accessed March 7, 2013).

(4) National Kidney and Urologic Diseases Information Clearinghouse. http://kidney.niddk.nih.gov/kudiseases/pubs/imagingut/ (accessed August 9, 2011).

(5) Bourin, P. M.; Jolliet, P.; Ballereau, F. An Overview of the Clinical Pharmacokinetics of X-Ray Contrast Media. Clinical-Pharmacokinetics 1997, 32, 180-193.

(6) Physiology or Medicine 1979 - Press Release. http://www.nobelprize.org/nobel_prizes/medicine/laureates/1979/press.html (accessed March 14, 2013).

(7) Goodman DM Initiatives focus on limiting radiation exposure to patients during ct scans. Journal of the American Medical Association 2013, 309, 647648. 
(8) US Department of Commerce, N. NIST: X-Ray Mass Attenuation

Coefficients. http://www.nist.gov/pml/data/xraycoef/index.cfm (accessed August 9, 2011).

(9) Cormode, D. P. Potential of multi-color computed tomography for advanced disease characterization. MEDICAMUNDI 2011, 55.

(10) Fromm, K. M. Barium bright and heavyNature Chemistry 2013, 5, 146146.

(11) Heiken, J. P.; Brink, J. A.; McClennan, B. L.; Sagel, S. S.; Crowe, T. M.; Gaines, M. V. Dynamic incremental CT: effect of volume and concentration of contrast material and patient weight on hepatic enhancement. Radiology 1995, 195, 353-357.

(12) Newington, I. M.; Humphries, G.; Lasbistes, N.; Morisson-Iveson, V.; Nairne, J.; Passmore, J.; Thanning, M.; Wistrand, L.-G.; Wynn, D. The synthesis and evaluation of trimeric X-ray contrast agents. Tetrahedron Letters 2011, 52, 3065-3067.

(13) Jean-Marc, I.; Emmanuelle, P.; Philippe, P.; Claire, C. Allergy-like reactions to iodinated contrast agents. A critical analysis. Fundamental \& Clinical Pharmacology 2005, 19, 263-281.

(14) Namasivayam, S.; Kalra, M. K.; Torres, W. E.; Small, W. C. Adverse reactions to intravenous iodinated contrast media: a primer for radiologists. Emergeny Radiology 2006, 12, 210-215. 
(15) Spring, D. B.; Bettmann, M. A.; Barkan, H. E. Nonfatal adverse reactions to iodinated contrast media: spontaneous reporting to the U.S. Food and Drug Administration, 1978-1994. Radiology 1997, 204, 325-332.

(16) Persson, P . B.; Hansell, P .; Liss, P . Pathophysiology of contrast medium- induced nephropathy. Kidney International 2005, 68, 14-22. (17) Solomon, R. The role of osmolality in the incidence of contrast-induced nephropathy: A systematic review of angiographic contrast media in high risk patients. Kidney International 2005, 68, 2256-2263.

(18) Hallouard, F.; Briançon, S.; Anton, N.; Li, X.; Vandamme, T.; Fessi, H. lodinated nano-emulsions as contrast agents for preclinical X-ray imaging: Impact of the free surfactants on the pharmacokinetics. European Journal of Pharmaceutics and Biopharmaceutics.

(19) Hallouard, F.; Anton, N.; Choquet, P.; Constantinesco, A.; Vandamme, T. lodinated blood pool contrast media for preclinical X-ray imaging applications - A review. Biomaterials 2010, 31, 6249-6268.

(20) Nune, S. K.; Gunda, P.; Thallapally, P. K.; Lin, Y.-Y.; Laird Forrest, M.; Berkland, C. J. Nanoparticles for biomedical imaging. Expert Opinion Drug Delivery 2009, 6, 1175-1194.

(21) Cho, E. C.; Glaus, C.; Chen, J.; Welch, M. J.; Xia, Y. Inorganic nanoparticle-based contrast agents for molecular imaging. Trends in Molecular Medicine 2010, 16, 561-573. 
(22) Lusic, H.; Grinstaff, M. W. X-ray-Computed Tomography Contrast Agents. Chemical Reviews. 2012.

(23) Choi, H. S.; Liu, W.; Misra, P.; Tanaka, E.; Zimmer, J. P.; Ipe, B. I.; Bawendi, M. G.; Frangioni, J. V. Renal Clearance of Nanoparticles. Nature Biotechnology 2007, 25, 1165-1170.

(24) Cai, Q.-Y.; Kim, S. H.; Choi, K. S.; Kim, S. Y.; Byun, S. J.; Kim, K. W.; Park, S. H.; Juhng, S. K.; Yoon, K.-H. Colloidal Gold Nanoparticles as a Blood- Pool Contrast Agent for X-ray Computed Tomography in Mice. Investigative Radiology 2007, 42, 797-806.

(25) Longmire, M.; Choyke, P. L.; Kobayashi, H. Clearance properties of nano- sized particles and molecules as imaging agents: considerations and caveats. Nanomedicine (Lond) 2008, 3, 703-717.

(26) Lee, N.; Choi, S. H.; Hyeon, T. Nano-Sized CT Contrast Agents. Advanced Materials 2013, 25, 2641-2660.

(27) Rabin, O.; Manuel Perez, J.; Grimm, J.; Wojtkiewicz, G.; Weissleder, R. An X-ray computed tomography imaging agent based on long-circulating bismuth sulphide nanoparticles. Nature Materials 2006, 5, 118-122.

(28) Kinsella, J. M.; Jimenez, R. E.; Karmali, P. P.; Rush, A. M.; Kotamraju, V. R.; Gianneschi, N. C.; Ruoslahti, E.; Stupack, D.; Sailor, M. J. X-Ray Computed Tomography Imaging of Breast Cancer by using Targeted Peptide-Labeled Bismuth Sulfide Nanoparticles. Angewandte Chemie International Edition. 
(29) Pan, D.; Roessl, E.; Schlomka, J.; Caruthers, S. D.; Senpan, A.; Scott, M. J.; Allen, J. S.; Zhang, H.; Hu, G.; Gaffney, P. J.; Choi, E. T.; Rasche, V.; Wickline, S. A.; Proksa, R.; Lanza, G. M. Computed Tomography in Color: NanoK-Enhanced Spectral CT Molecular Imaging. Angewandte Chemie 2010, 122, 9829-9833.

(30) Pan, D.; Williams, T. A.; Senpan, A.; Allen, J. S.; Scott, M. J.; Gaffney, P. J.; Wickline, S. A.; Lanza, G. M. Detecting Vascular Biosignatures with a Colloidal, Radio-Opaque Polymeric Nanoparticle. Journal of the American Chemical Society 2009, 131, 15522-15527.

(31) Hainfeld, J. F.; Slatkin, D. N.; Focella, T. M.; Smilowitz, H. M. Gold nanoparticles: a new X-ray contrast agent. British Journal of Radiology 2006, $79,248-253$.

(32) Ahn, S.; Jung, S.; Lee, S. Gold Nanoparticle Contrast Agents in Advanced X-ray Imaging Technologies. Molecules 2013, 18, 5858-5890. (33) Liu, Z.; Li, Z.; Liu, J.; Gu, S.; Yuan, Q.; Ren, J.; Qu, X. Long-circulating Er3+-doped Yb2O3 up-conversion nanoparticle as an in vivo X-Ray CT imaging contrast agent. Biomaterials 2012, 33, 6748-6757.

(34) Torres, A. S.; Bonitatibus, P. J.; Colborn, R. E.; Goddard, G. D.; FitzGerald, P. F.; Lee, B. D.; Marino, M. E. Biological Performance of a SizeFractionated Core-Shell Tantalum Oxide Nanoparticle X-Ray Contrast Agent. Investigative Radiology 2012, 47, 578-587. 
(35) Bonitatibus, P. J.; Torres, A. S.; Kandapallil, B.; Lee, B. D.; Goddard, G. D.; Colborn, R. E.; Marino, M. E. Preclinical Assessment of a Zwitterionic Tantalum Oxide Nanoparticle X-ray Contrast Agent. ACS Nano 2012, 6, 6650-6658.

(36) Kim, D.; Park, S.; Lee, J. H.; Jeong, Y. Y.; Jon, S. Antibiofouling Polymer- Coated Gold Nanoparticles as a Contrast Agent for in Vivo X-ray Computed Tomography Imaging. Journal of the American Chemical Society 2007, 129, 7661-7665.

(37) Popovtzer, R.; Agrawal, A.; Kotov, N. A.; Popovtzer, A.; Balter, J.; Carey, T. E.; Kopelman, R. Targeted Gold Nanoparticles enable Molecular CT Imaging of Cancer. Nano Lett 2008, 8, 4593-4596. 56

(38) Liu, Y.; Ai, K.; Lu, L. Nanoparticulate X-ray Computed Tomography Contrast Agents: From Design Validation to in Vivo Applications. Accounts of Chemical Research 2012.

(39) Khlebtsov, N.; Dykman, L. Biodistribution and toxicity of engineered gold nanoparticles: a review of in vitro and in vivo studies. Chemical Society Reviews 2011, 40, 1647-1671.

(40) Lasagna-Reeves, C.; Gonzalez-Romero, D.; Barria, M. A.; Olmedo, I.; Clos, A.; Sadagopa Ramanujam, V. M.; Urayama, A.; Vergara, L.; Kogan, M. J.; Soto, C. Bioaccumulation and toxicity of gold nanoparticles after repeated administration in mice. Biochemical and Biophysical Research Communications 2010, 393, 649-655. 
(41) De Marcillac, P.; Coron, N.; Dambier, G.; Leblanc, J.; Moalic, J.-P. Experimental detection of [alpha]-particles from the radioactive decay of natural bismuth. Nature 2003, 422, 876-878.

(42) Bismuth metal prices, Bismuth metal ingot prices, Bismuth charts, Bismuth historical prices, Business graphs.

http://www.metalprices.com/FreeSite/metals/bi/bi.asp (accessed September 7, 2011).

(43) Briand, G. G.; Burford, N. Bismuth compounds and preparations with biological or medicinal relevance. Chemical Review 1999, 99, 2601-2658.

(44) Bierer, D. W. Bismuth subsalicylate: history, chemistry, and safety. Review of Infections Diseases 1990, 12 Suppl 1, S3-8. 57

(45) Lee, S. P. The mode of action of colloidal bismuth subcitrate. Scandinavian Journal of Gastroenterology 1991, 185, 1-6. (46) Lambert, J. R.; Midolo, P. The actions of bismuth in the treatment of Helicobacter pylori infection. Alimentary Pharmacology \& Therapeutics 1997, 11, 27-33.

(47) Lambert, J. R. Pharmacology of Bismuth-Containing Compounds. Clinical Infectious Diseases 1991, 13, S691-S695.

(48) Serfontein, W. J.; Mekel, R. Bismuth toxicity in man II. Review of bismuth blood and urine levels in patients after administration of therapeutic bismuth formulations in relation to the problem of bismuth toxicity in man. 
Research Communications Chemical Pathology and Pharmacology 1979, 26, $391-411$.

(49) Sadler, P. J.; Li, H.; Sun, H. Coordination chemistry of metals in medicine: target sites for bismuth. Coordination Chemistry Reviews 1999, 185-186, 689-709.

(50) A. Dahlgren, C. Gløgård, M. Gammels Organobismuth Compounds: Activity against Helicobacter pylori. Scandinavian Journal of Gastroenterology 1999, 34, 135-137.

(51) Stratton, C. W.; Warner, R. R.; Coudron, P. E.; Lilly, N. A. Bismuthmediated disruption of the glycocalyx- cell wall of Helicobacter pylori:ultrastructural evidence for a mechanism of action for bismuth salts. Journal of Antimicrobical Chemotherapy 1999, 43, 659-666.

(52) Wagner, S.; Beil, W.; Mai, U.E.H.; Bokemeyer, C.; Meyer, H.J.; Manns, M.P. Interaction between Helicobacter pylori and human gastric epithelial cells in culture: effect of antiulcer drugs. Pharmacology 1994, 49, 226-237. (53) Vanhoe, H.; Versieck, J.; Vanballenberghe, L.; Dams, R. Bismuth in human serum: reference interval and concentrations after intake of a therapeutic dose of colloidal bismuth subcitrateClinical Chimica Acta 1993, 219, 79-91.

(54) Nwokolo, C. U.; Gavey, C. J.; Smith, J. T. L.; Pounder, R. E. The absorption of bismuth from oral doses of tripotassium dicitrato bismuthate. Alimentary Pharmacology \& Therapeutics 1989, 3, 29-39. 
(55) Hudson, M.; Mowat, N. A. Reversible toxicity in poisoning with colloidal bismuth subcitrate. BMJ : British Medical Journal 1989, 299, 159.

(56) Bradley, B.; Singleton, M.; Po, A. L. W. Bismuth Toxicity-a

Reassessment ${ }^{\star}$. Journal of Clinical Pharmacy and Therapeutics 1989, 14, 423-441.

(57) Sano, Y.; Satoh, H.; Chiba, M.; Okamoto, M.; Serizawa, K.; Nakashima, H.; Omae, K. Journal of Occupational Health 2005, 47, 293-298.

(58) Zhao, Y.; Zhang, Z.; Dang, H. A simple way to prepare bismuth nanoparticles. Materials Letters 2004, 58, 790-793.

(59) Wang, Y.; Xia, Y. Bottom-Up and Top-Down Approaches to the Synthesis of Monodispersed Spherical Colloids of Low Melting-Point Metals. Nano Letters 2004, 4, 2047-2050.

(60) Wang, F.; Tang, R.; Yu, H.; Gibbons, P. C.; Buhro, W. E. Size- and Shape- Controlled Synthesis of Bismuth Nanoparticles. Chemistry of Materials 2008, 20, 3656-3662.

(61) Yarema, M.; Kovalenko, M. V.; Hesser, G.; Talapin, D. V.; Heiss, W. Highly Monodisperse Bismuth Nanoparticles and Their Three-Dimensional Superlattices. Journal of the American Chemical Society 2010, 132, 1515815159.

(62) Richards, V. N.; Shields, S. P.; Buhro, W. E. Nucleation Control in the Aggregative Growth of Bismuth Nanocrystals. Chemistry of Materials 2011, 23, $137-144$. 
(63) Wang, F.; Buhro, W. E. An easy shortcut synthesis of size-controlled bismuth nanoparticles and their use in the SLS growth of high-quality colloidal cadmium selenide quantum wires. Small 2010, 6, 573-581.

(64) Schulz, S.; Heimann, S.; Wölper, C.; Assenmacher, W. Synthesis of Bismuth Pseudocubes by Thermal Decomposition of Bi2Et4. Chemistry of Materials 2012, 24, 2032-2039.

(65) Warren, S. C.; Jackson, A. C.; Cater-Cyker, Z. D.; DiSalvo, F. J.; Wiesner, U. Nanoparticle Synthesis via the Photochemical Polythiol Process. Journal of the American Chemical Society 2007, 129, 10072-10073.

(66) Wang, Y .; Chen, J.; Chen, L.; Chen, Y .-B.; Wu, L.-M. Shape-Controlled Solventless Syntheses of Nano Bi Disks and Spheres. Crystal Growth \& Design 2010, 10, 1578-1584.

(67) Son, J. S.; Park, K.; Han, M.; Kang, C.; Park, S.; Kim, J.; Kim, W.; Kim, S.; Hyeon, T. Large-Scale Synthesis and Characterization of the Size-Dependent Thermoelectric Properties of Uniformly Sized Bismuth Nanocrystals. Angewandte Chemie International Edition 2011, 50, 13631366.

(68) Wang, Y. W.; Hong, B. H.; Kim, K. S. Size Control of Semimetal Bismuth Nanoparticles and the UV-Visible and IR Absorption Spectra. The Journal of Physical Chemistry B 2005, 109, 7067-7072. 
(69) Hossain, M.; Luo, Y.; Sun, Z.; Wang, C.; Zhang, M.; Fu, H.; Qiao, Y.; Su, M. X-ray enabled detection and eradication of circulating tumor cells with nanoparticles. Biosensors and Bioelectronics 2012, 38, 348-354.

(70) Luo, Y.; Wang, C.; Qiao, Y.; Hossain, M.; Ma, L.; Su, M. In vitro cytotoxicity of surface modified bismuth nanoparticles. Journal of Materials Science: Materials in Medicine 1-11.

(71) Fievet, F.; Lagier, J.; Blin, B.; Beaudoin, B.; Figlarz, M. Homogeneous and heterogeneous nucleations in the polyol process for the preparation of micron and submicron size metal particles. Solid State lonics 1989, 32-33, 198-205.

(72) Goia, C.; Matijević, E.; Goia, D. V. Preparation of Colloidal Bismuth Particles in Polyols. Journal of Materials Research 2011, 20, 1507-1514. (73) Wang, Y.; Kim, K. S. Large-scale polyol synthesis of single-crystal bismuth nanowires and the role of $\mathrm{NaOH}$ in the synthesis process. Nanotechnology 2008, 19, 265303.

(74) Wang, J.; Wang, X.; Peng, Q.; Li, Y. Synthesis and Characterization of Bismuth Single-Crystalline Nanowires and Nanospheres. Inorganic Chemistry 2004, 43, 7552-7556.

(75) Li, J.; Fan, H.; Chen, J.; Liu, L. Synthesis and characterization of poly(vinyl pyrrolidone)-capped bismuth nanospheres. Colloids and Surfaces A: Physicochemical and Engineering Aspects 2009, 340, 66-69. 
(76) Zou, C. D.; Gao, Y. L.; Yang, B.; Zhai, Q. J. Melting and undercooling of bismuth nanocrystals by solvothermal synthesis. Physica B: Condensed Matter 2009, 404, 4045-4050.

(77) Wang, W. Z.; Poudel, B.; Ma, Y.; Ren, Z. F. Shape Control of Single Crystalline Bismuth Nanostructures. The Journal of Physical Chemistry $B$ 2006, 110, 25702-25706.

(78) Cheng, G.; Wu, J.; Xiao, F.; Yu, H.; Lu, Z.; Yu, X.; Chen, R. Synthesis of bismuth micro- and nanospheres by a simple refluxing method. Materials Letters 2009, 63, 2239-2242.

(79) Chen, Y.; Gong, R.; Zhang, W.; Xu, X.; Fan, Y.; Liu, W. Synthesis of single- crystalline bismuth nanobelts and nanosheets. Materials Letters 2005, $59,909-911$.

(80) Foos, E. E. Synthesis of Nanocrystalline Bismuth in Reverse Micelles. Journal of the American Chemical Society 122, 7114-7115.

(81) Fang, J.; Stokes, K. L.; Zhou, W. L.; Wiemann, J. A.; Dai, J.; Oconnor, C. J. COLLOIDAL BISMUTH NANOPARTICLES: SYNTHESIS AND UV-VIS ABSORPTION. World Scientific Publishing Co. Pte. Ltd., 2000; pp. 91-96. (82) Fang, J.; Stokes, K. L.; Wiemann, J. A.; Zhou, W. L.; Dai, J.; Chen, F.; O'Connor, C. J. Microemulsion-processed bismuth nanoparticles. Materials Science and Engineering B 2001, 83, 254-257. 
(83) Gutierrez, M.; Henglein, A. Nanometer-Sized Bi Particles in Aqueous Solution: Absorption Spectrum and Some Chemical Properties. Journal of Physical Chemistry 1996 100(18), 7656-7661

(84) Wang, Y .; Zhao, J.; Zhao, X.; T ang, L.; Li, Y .; Wang, Z. A facile waterbased process for preparation of stabilized Bi nanoparticles. Materials Research Bulletin 2009, 44, 220-223.

(85) Ma, D.; Zhao, J.; Zhao, Y.; Hao, X.; Li, L.; Zhang, L.; Lu, Y.; Yu, C.

Synthesis of bismuth nanoparticles and self-assembled nanobelts by a simple aqueous route in basic solution. Colloids and Surfaces A: Physicochemical and Engineering Aspects 2012, 395, 276-283.

(86) Fang, J.; Stokes, K. L.; Wiemann, J.; Zhou, W. Nanocrystalline bismuth synthesized via an in situ polymerization-microemulsion process. Materials Letters 2000, 42, 113-120. 


\section{References from Chapter 2:}

(1) Zhang, Z.; Ying, J. Y.; Dresselhaus, M. S. Bismuth Quantum-Wire Arrays Fabricated by a Vacuum Melting and Pressure Injection Process. Journal of Materials Research 1998, 13, 1745-1748.

(2) Wang, Y. W.; Kim, J. S.; Kim, G. H.; Kim, K. S. Quantum size effects in the volume plasmon excitation of bismuth nanoparticles investigated by electron energy loss spectroscopy. Applied Physics Letters 2006, 88, 143106.

(3) Heremans, J.; Thrush, C. M. Thermoelectric power of bismuth nanowires. Physical Review B 1999, 59, 12579.

(4) Boukai, A.; Xu, K.; Heath, J. R. Size-Dependent Transport and Thermoelectric Properties of Individual Polycrystalline Bismuth Nanowires. Advanced Materials 2006, 18, 864-869.

(5) Fanfair, D. D.; Korgel, B. A. Bismuth Nanocrystal-Seeded III-V Semiconductor Nanowire Synthesis. Crystal Growth \& Design 2005, 5, 19711976.

(6) Wang, F.; Tang, R.; Kao, J. L.-F.; Dingman, S. D.; Buhro, W. E. Spectroscopic Identification of Tri-n-octylphosphine Oxide (TOPO) Impurities and Elucidation of Their Roles in Cadmium Selenide Quantum- Wire Growth. Journal of the American Chemical Society 2009, 131, 4983- 4994.

(7) Chockla, A. M.; Harris, J. T.; Korgel, B. A. Colloidal Synthesis of Germanium Nanorods. Chemistry of Materials 2011, 23, 1964-1970. 
(8) Rabin, O.; Manuel Perez, J.; Grimm, J.; Wojtkiewicz, G.; Weissleder, R. An X-ray computed tomography imaging agent based on long-circulating bismuth sulphide nanoparticles. Nature Materials 2006, 5, 118-122.

(9) Pan, D.; Roessl, E.; Schlomka, J.; Caruthers, S. D.; Senpan, A.; Scott, M. J.; Allen, J. S.; Zhang, H.; Hu, G.; Gaffney, P. J.; Choi, E. T.; Rasche, V.; Wickline, S. A.; Proksa, R.; Lanza, G. M. Computed Tomography in Color: NanoK-Enhanced Spectral CT Molecular Imaging. Angewandte Chemie 2010, 122, 9829-9833.

(10) Ai, K.; Liu, Y.; Liu, J.; Yuan, Q.; He, Y.; Lu, L. Large-Scale Synthesis of Bi2S3 Nanodots as a Contrast Agent for In Vivo X-ray Computed Tomography Imaging. Advanced Materials 2011, 23, 4886-4891.

(11) Choi, H. S.; Liu, W.; Misra, P.; Tanaka, E.; Zimmer, J. P.; Ipe, B. I.; Bawendi, M. G.; Frangioni, J. V. Renal Clearance of Nanoparticles. Nature Biotechnol 2007, 25, 1165-1170.

(12) Mammen, M.; Choi, S.; Whitesides, G. M. Polyvalent Interactions in Biological Systems: Implications for Design and Use of Multivalent Ligands and Inhibitors. Angewandte Chemie International Edition 1998, 37, 2754- 2794. (13) Briand, G. G.; Burford, N. Bismuth compounds and preparations with biological or medicinal relevance. Chemical Reviews 1999, 99, 2601-2658. (14) Hainfeld, J. F.; Slatkin, D. N.; Focella, T. M.; Smilowitz, H. M. Gold nanoparticles: a new X-ray contrast agent. British Journal of Radiology 2006, 79, 248-253. 
(15) Popovtzer, R.; Agrawal, A.; Kotov, N. A.; Popovtzer, A.; Balter, J.; Carey, T. E.; Kopelman, R. Targeted Gold Nanoparticles enable Molecular CT Imaging of Cancer. Nano Letters 2008, 8, 4593-4596.

(16) Eck, W.; Nicholson, A. I.; Zentgraf, H.; Semmler, W.; Bartling, S. Anti-CD4targeted Gold Nanoparticles Induce Specific Contrast Enhancement of Peripheral Lymph Nodes in X-ray Computed Tomography of Live Mice. Nano Letters 2010, 10, 2318-2322.

(17) Free Bismuth Price Charts. http://www.metalprices.com/pubcharts/Public/Bismuth_Price_Charts.asp (accessed August 8, 2011).

(18) Greenwood, N.N.; Earnshaw, A. In Chemistry of the elements, Second Edition; Pergamon Press: Oxford, UK, 1998; Vol. Arsenic, Antimony and Bismuth.

(19) Cormode, D. P.; Skajaa, T.; Fayad, Z. A.; Mulder, W. J. M. Nanotechnology in Medical Imaging. Arteriosclerosis, Thrombosis, and Vascular Biology 2009, 29, $992-1000$.

(20) Longmire, M.; Choyke, P. L.; Kobayashi, H. Clearance properties of nanosized particles and molecules as imaging agents: considerations and caveats. Nanomedicine (Lond) 2008, 3, 703-717.

(21) Bierer, D. W. Bismuth subsalicylate: history, chemistry, and safety. Reviews of Infectious Diseases 1990, 12 Supplement 1, S3-8. 
(22) Sun, H.; Li, H.; Harvey, I.; Sadler, P. J. Interactions of bismuth complexes with metallothionein(II). Journal Biological Chemistry 1999, 274, 29094- 29101. (23) Yu, H.; Gibbons, P. C.; Buhro, W. E. Bismuth, tellurium, and bismuth telluride nanowires Journal Materials Chemistry 2004, 14, 595.

(24) Wang, F.; Tang, R.; Yu, H.; Gibbons, P. C.; Buhro, W. E. Size- and ShapeControlled Synthesis of Bismuth Nanoparticles. Chemistry of Materials 2008, 20, $3656-3662$.

(25) Richards, V. N.; Shields, S. P.; Buhro, W. E. Nucleation Control in the Aggregative Growth of Bismuth Nanocrystals. Chemistry of Materials 2011, 23, 137-144.

(26) Wang, Y.; Xia, Y. Bottom-Up and Top-Down Approaches to the Synthesis of Monodispersed Spherical Colloids of Low Melting-Point Metals. Nano Letters 2004, 4, 2047-2050.

(27) Li, J.; Fan, H.; Chen, J.; Liu, L. Synthesis and characterization of poly(vinyl pyrrolidone)-capped bismuth nanospheres. Colloids and Surfaces A:

Physicochemical and Engineering Aspects 2009, 340, 66-69.

(28) Wang, F.; Tang, R.; Yu, H.; Gibbons, P. C.; Buhro, W. E. Size- and ShapeControlled Synthesis of Bismuth Nanoparticles. Chemistry of Materials 2008, 20, $3656-3662$.

(29) Wang, Y.; Kim, K. S. Large-scale polyol synthesis of single-crystal bismuth

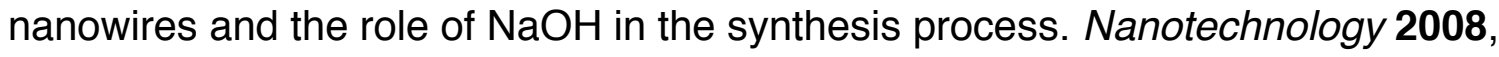
19, 265303. 
(30) Wang, Y.; Zhao, J.; Zhao, X.; Tang, L.; Li, Y.; Wang, Z. A facile water-based process for preparation of stabilized Bi nanoparticles. Materials Research Bulletin 2009, 44, 220-223.

(31) Irmawati, R.; Noorfarizan Nasriah, M. N.; Taufiq-Yap, Y. H.; Abdul Hamid, S. B. Characterization of bismuth oxide catalysts prepared from bismuth trinitrate pentahydrate: influence of bismuth concentration. Catalysis Today 2004, 93-95, 701-709.

(32) Kragten, J.; Decnop-Weever, L. G.; Gründler, P. Mixed hydroxide complex formation and solubility of bismuth in nitrate and perchlorate medium. Talanta 1993, 40, 485-490.

(33) Schumb, W. C.; Rittner, E. S. Polymorphism of Bismuth Trioxide1. Journal of the American Chemical Society 1943, 65, 1055-1060.

(34) Wulfsberg, G. Inorganic chemistry; University Science Books, 2000.

(35) Taylor, P.; Lopata, V. J. Some phase relationships between basic bismuth chlorides in aqueous solutions at $25^{\circ} \mathrm{C}$. Canadian Journal of Chemistry 1987, 65, 2824-2829.

(36) Kinomura, N.; Kumada, N. Preparation of bismuth oxides with mixed valence from hydrated sodium bismuth oxide. Materials Research Bulletin 1995, 30, 129-134.

(37) Suzuki, S.; Shimanouchi, T.; Tsuboi, M. Normal vibrations of glycine and deuterated glycine molecules. Spectrochimica Acta 1963, 19, 1195-1208. (38) Yarema, M.; Kovalenko, M. V.; Hesser, G.; Talapin, D. V.; Heiss, W. Highly 
Monodisperse Bismuth Nanoparticles and Their Three-Dimensional

Superlattices. Journal of the American Chemical Society 2010, 132, 1515815159.

\section{References from Chapter 3:}

(1) Choi, H. S.; Liu, W.; Misra, P.; Tanaka, E.; Zimmer, J. P.; Ipe, B. I.; Bawendi, M. G.; Frangioni, J. V. Renal Clearance of Nanoparticles. Nature Biotechnology 2007, 25, 1165-1170.

(2) Jakhmola, A.; Anton, N.; Vandamme, T. F. Inorganic Nanoparticles Based Contrast Agents for X-ray Computed Tomography. Advanced Healthcare Materials 2012, 1, 413-431.

(3) Debbage, P.; Jaschke, W. Molecular imaging with nanoparticles: giant roles for dwarf actors. Histochemistry and Cellular Biology 2008, 130, 845-875. (4) Ahn, S.; Jung, S.; Lee, S. Gold Nanoparticle Contrast Agents in Advanced Xray Imaging Technologies. Molecules 2013, 18, 5858-5890.

(5) Cai, Q.-Y.; Kim, S. H.; Choi, K. S.; Kim, S. Y.; Byun, S. J.; Kim, K. W.; Park, S. H.; Juhng, S. K.; Yoon, K.-H. Colloidal Gold Nanoparticles as a Blood-Pool Contrast Agent for X-ray Computed Tomography in Mice. Investigative Radiology 2007, 42, 797-806.

(6) Chien, C.-C.; Chen, H.-H.; Lai, S.-F.; Hwu, Y.; Petibois, C.; Yang, C. S.; Chu, Y.; Margaritondo, G. X-ray imaging of tumor growth in live mice by detecting gold-nanoparticle-loaded cells. Scientific Reports 2012, 2. 
(7) Eck, W.; Nicholson, A. I.; Zentgraf, H.; Semmler, W.; Bartling, S. Anti-CD4targeted Gold Nanoparticles Induce Specific Contrast Enhancement of Peripheral Lymph Nodes in X-ray Computed Tomography of Live Mice. Nano Letters 2010, 10, 2318-2322.

(8) Hainfeld, J. F.; Smilowitz, H. M.; O'Connor, M. J.; Dilmanian, F. A.; Slatkin, D. N. Gold nanoparticle imaging and radiotherapy of brain tumors in mice. Nanomedicine 2012, 1-9.

(9) Xu, C.; Tung, G. A.; Sun, S. Size and Concentration Effect of Gold Nanoparticles on X-ray Attenuation As Measured on Computed Tomography. Chemistry Materials 2008, 20, 4167-4169.

(10) Live Gold, Silver, Platinum, Palladium Quote Spot Price Chart - Kitco. http://www.kitco.com/market/ (accessed June 6, 2013).

(11) Lasagna-Reeves, C.; Gonzalez-Romero, D.; Barria, M. A.; Olmedo, I.; Clos, A.; Sadagopa Ramanujam, V. M.; Urayama, A.; Vergara, L.; Kogan, M. J.; Soto, C. Bioaccumulation and toxicity of gold nanoparticles after repeated administration in mice. Biochemical and Biophysical Research Communications 2010, 393, 649-655.

(12) Khlebtsov, N.; Dykman, L. Biodistribution and toxicity of engineered gold nanoparticles: a review of in vitro and in vivo studies. Chemical Society Review 2011, 40, 1647-1671.

(13) Pan, Y.; Leifert, A.; Ruau, D.; Neuss, S.; Bornemann, J.; Schmid, G.;

Brandau, W.; Simon, U.; Jahnen-Dechent, W. Gold Nanoparticles of Diameter 1.4 
nm Trigger Necrosis by Oxidative Stress and Mitochondrial Damage. Small 2009, 5, 2067-2076.

(14) Bismuth metal prices, news, charts and historical prices.

http://www.metalprices.com/metal/bismuth (accessed June 6, 2013).

(15) Greenwood, N.N.; Earnshaw, A. In Chemistry of the elements, Second Edition; Pergamon Press: Oxford, UK, 1998; Vol. Arsenic, Antimony and Bismuth.

(16) Sano, Y.; Satoh, H.; Chiba, M.; Okamoto, M.; Serizawa, K.; Nakashima, H.; Omae, K. Journal of Occupational Health 2005, 47, 293-298.

(17) Serfontein, W. J.; Mekel, R. Bismuth toxicity in man II. Review of bismuth blood and urine levels in patients after administration of therapeutic bismuth formulations in relation to the problem of bismuth toxicity in man. Research Communications in Chemical Pathology and Pharmacology 1979, 26, $391-411$.

(18) Briand, G. G.; Burford, N. Bismuth compounds and preparations with biological or medicinal relevance. Chemical Reviews 1999, 99, 2601-2658.

(19) Rabin, O.; Manuel Perez, J.; Grimm, J.; Wojtkiewicz, G.; Weissleder, R. An X-ray computed tomography imaging agent based on long-circulating bismuth sulphide nanoparticles. Nature Materials 2006, 5, 118-122.

(20) Kinsella, J. M.; Jimenez, R. E.; Karmali, P. P.; Rush, A. M.; Kotamraju, V. R.; Gianneschi, N. C.; Ruoslahti, E.; Stupack, D.; Sailor, M. J. X-Ray Computed 
Tomography Imaging of Breast Cancer by using Targeted Peptide-Labeled Bismuth Sulfide Nanoparticles. Angewandte Chemie International Edition. (21) Pan, D.; Roessl, E.; Schlomka, J.; Caruthers, S. D.; Senpan, A.; Scott, M. J.; Allen, J. S.; Zhang, H.; Hu, G.; Gaffney, P. J.; Choi, E. T.; Rasche, V.; Wickline, S. A.; Proksa, R.; Lanza, G. M. Computed Tomography in Color: NanoK-Enhanced Spectral CT Molecular Imaging. Angewandte Chemie 2010, 122, 9829-9833.

(22) Luo, Y.; Wang, C.; Hossain, M.; Qiao, Y.; Ma, L.; An, J.; Su, M. ThreeDimensional Microtissue Assay for High-Throughput Cytotoxicity of Nanoparticles. Analitical Chemistry 2012, 84, 6731-6738.

(23) Hossain, M.; Luo, Y.; Sun, Z.; Wang, C.; Zhang, M.; Fu, H.; Qiao, Y.; Su, M. X-ray enabled detection and eradication of circulating tumor cells with nanoparticles. Biosensors and Bioelectronics 2012, 38, 348-354.

(24) Yarema, M.; Kovalenko, M. V.; Hesser, G.; Talapin, D. V.; Heiss, W. Highly Monodisperse Bismuth Nanoparticles and Their Three-Dimensional Superlattices. Journal of the American Chemical Society 2010, 132, 1515815159.

(25) Fang, J.; Stokes, K. L.; Wiemann, J.; Zhou, W. Nanocrystalline bismuth synthesized via an in situ polymerization-microemulsion process. Materials Letters 2000, 42, 113-120. 
(26) Warren, S. C.; Jackson, A. C.; Cater-Cyker, Z. D.; DiSalvo, F. J.; Wiesner, U. Nanoparticle Synthesis via the Photochemical Polythiol Process. Journal of the American Chemical Society 2007, 129, 10072-10073.

(27) Wang, Y. W.; Hong, B. H.; Kim, K. S. Size Control of Semimetal Bismuth Nanoparticles and the UV-Visible and IR Absorption Spectra. Journal of Physical Chemistry B 2005, 109, 7067-7072.

(28) Wang, F.; Buhro, W. E. An easy shortcut synthesis of size-controlled bismuth nanoparticles and their use in the SLS growth of high-quality colloidal cadmium selenide quantum wires. Small 2010, 6, 573-581.

(29) Richards, V. N.; Shields, S. P.; Buhro, W. E. Nucleation Control in the Aggregative Growth of Bismuth Nanocrystals. Chemistry of Materials 2011, 23, 137-144.

(30) Wang, F.; Tang, R.; Yu, H.; Gibbons, P. C.; Buhro, W. E. Size- and ShapeControlled Synthesis of Bismuth Nanoparticles. Chemistry of Materials 2008, 20, 3656-3662.

(31) Schulz, S.; Heimann, S.; Wölper, C.; Assenmacher, W. Synthesis of Bismuth Pseudocubes by Thermal Decomposition of Bi2Et4. Chemistry of Materials 2012, 24, 2032-2039.

(32) Wang, Y.; Kim, K. S. Large-scale polyol synthesis of single-crystal bismuth nanowires and the role of $\mathrm{NaOH}$ in the synthesis process. Nanotechnology 2008, 19, 265303. 
(33) Goia, C.; Matijević, E.; Goia, D. V. Preparation of Colloidal Bismuth Particles in Polyols. Journal of Materials Research 2011, 20, 1507-1514.

(34) Wang, Y.; Xia, Y. Bottom-Up and Top-Down Approaches to the Synthesis of Monodispersed Spherical Colloids of Low Melting-Point Metals. Nano Letters 2004, 4, 2047-2050.

(35) Sadler, P. J.; Li, H.; Sun, H. Coordination chemistry of metals in medicine: target sites for bismuth. Coordination Chemistry Reviews 1999, 185-186, 689709.

(36) Sun, H.; Li, H.; Harvey, I.; Sadler, P. J. Interactions of bismuth complexes with metallothionein(II). Journal of Biological Chemistry 1999, 274, 2909429101.

(37) Phillips, H. A.; Eelman, M. D.; Burford, N. Cooperative influence of thiolate ligands on the bio-relevant coordination chemistry of bismuth. Journal of Inorganic Biochemistry 2007, 101, 736-739.

(38) Brown, A. L.; Goforth, A. M. pH-Dependent Synthesis and Stability of Aqueous, Elemental Bismuth Glyconanoparticle Colloids: Potentially Biocompatible X-ray Contrast Agents. Chemistry of Materials 2012.

(39) Wang, Y .; Zhao, J.; Zhao, X.; T ang, L.; Li, Y .; Wang, Z. A facile waterbased process for preparation of stabilized Bi nanoparticles. Materials Research Bulletin 2009, 44, 220-223.

(40) Ma, D.; Zhao, J.; Zhao, Y.; Hao, X.; Li, L.; Zhang, L.; Lu, Y.; Yu, C.

Synthesis of bismuth nanoparticles and self-assembled nanobelts by a simple 
aqueous route in basic solution. Colloids and Surfaces A: Physicochemical and Engineering Aspects 2012, 395, 276-283.

(41) Galper, M. W.; Saung, M. T.; Fuster, V.; Roessl, E.; Thran, A.; Proksa, R.; Fayad, Z. A.; Cormode, D. P. Effect of computed tomography scanning parameters on gold nanoparticle and iodine contrast. Investigative Radiology 2012, 47, 475-481.

(42) Cormode, D. P.; Skajaa, T.; van Schooneveld, M. M.; Koole, R.; Jarzyna, P.; Lobatto, M. E.; Calcagno, C.; Barazza, A.; Gordon, R. E.; Zanzonico, P.; Fisher, E. A.; Fayad, Z. A.; Mulder, W. J. M. Nanocrystal Core High-Density Lipoproteins: A Multimodality Contrast Agent Platform. Nano Letters 2008, 8, 3715-3723. (43) Hirn, S.; Semmler-Behnke, M.; Schleh, C.; Wenk, A.; Lipka, J.; Schäffler, M.; Takenaka, S.; Möller, W.; Schmid, G.; Simon, U.; Kreyling, W. G. Particle size-dependent and surface charge-dependent biodistribution of gold nanoparticles after intravenous administration. European Journal of Pharmaceutics and Biopharmaceutics 2011, 77, 407-416.

(44) Alexis, F.; Pridgen, E.; Molnar, L. K.; Farokhzad, O. C. Factors Affecting the Clearance and Biodistribution of Polymeric Nanoparticles. Molecular Pharmaceutics 2008, 5, 505-515.

(45) Wang, J.; Wang, X.; Peng, Q.; Li, Y. Synthesis and Characterization of Bismuth Single-Crystalline Nanowires and Nanospheres. Inorganic Chemistry 2004, 43, 7552-7556. 
(46) Wang, W. Z.; Poudel, B.; Ma, Y.; Ren, Z. F. Shape Control of Single Crystalline Bismuth Nanostructures. The Journal of Physical Chemistry B 2006, 110, 25702-25706.

(47) Cheng, G.; Wu, J.; Xiao, F.; Yu, H.; Lu, Z.; Yu, X.; Chen, R. Synthesis of bismuth micro- and nanospheres by a simple refluxing method. Materials Letters 2009, 63, 2239-2242.

(48) Segur, J. B.; Oberstar, H. E. Viscosity of Glycerol and Its Aqueous Solutions. Industrial and Engineering Chemistry Research. 1951, 43, 21172120.

(49) Saleh, M. A.; Begum, S.; Begum, S. K.; Begum, B. A. Viscosity of Dilute Aqueous Solutions of Some Diols. Physics and Chemistry of Liquids 1999, 37, 785-801.

(50) Huff, E. The metabolism of 1,2-propanediol. Biochimica et Biophysica Acta $1961,48,506-517$.

(51) El-Haes, H.; Leon, A. de; Jalbout, A. F.; Alaam, M.; Ibrahim, M. Analysis of the structure and vibrational spectra of glucose and fructose. Eclética Química 2006, 31, 15-21.

(52) Oh, S. Y.; Yoo, D. I.; Shin, Y.; Seo, G. FTIR analysis of cellulose treated with sodium hydroxide and carbon dioxide. Carbohydrate Research 2005, 340, 417-428. 


\section{References from Chapter 4:}

(1) Boukai, A.; Xu, K.; Heath, J. R. Size-Dependent Transport and

Thermoelectric Properties of Individual Polycrystalline Bismuth Nanowires. Advanced Materials 2006, 18, 864-869.

(2) Carotenuto, G.; Hison, C. L.; Capezzuto, F.; Palomba, M.; Perlo, P.; Conte, P. Synthesis and thermoelectric characterisation of bismuth nanoparticles. Journal of Nanoparticle Research 2008, 11, 1729-1738.

(3) Hostler, S. R.; Qu, Y. Q.; Demko, M. T.; Abramson, A. R.; Qiu, X.; Burda, C. Thermoelectric properties of pressed bismuth nanoparticles. Superlattices and Microstructures 2008, 43, 195-207.

(4) Son, J. S.; Park, K.; Han, M.; Kang, C.; Park, S.; Kim, J.; Kim, W.; Kim, S.; Hyeon, T. Large-Scale Synthesis and Characterization of the Size-Dependent Thermoelectric Properties of Uniformly Sized Bismuth Nanocrystals. Angewandte Chemie International Edition 2011, 50, 13631366.

(5) Heremans, J.; Thrush, C. M. Thermoelectric power of bismuth nanowires. Phys. Rev. B 1999, 59, 12579.

(6) Rabin, O.; Manuel Perez, J.; Grimm, J.; Wojtkiewicz, G.; Weissleder, R. An X-ray computed tomography imaging agent based on long-circulating bismuth sulphide nanoparticles. Nature Materials 2006, 5, 118-122. 
(7) Brown, A. L.; Goforth, A. M. pH-Dependent Synthesis and Stability of Aqueous, Elemental Bismuth Glyconanoparticle Colloids: Potentially Biocompatible X-ray Contrast Agents. Chemistry Materials 2012.

(8) Wang, F.; Tang, R.; Yu, H.; Gibbons, P. C.; Buhro, W. E. Size- and ShapeControlled Synthesis of Bismuth Nanoparticles. Chemistry of Materials 2008, 20, 3656-3662.

(9) Richards, V. N.; Shields, S. P.; Buhro, W. E. Nucleation Control in the Aggregative Growth of Bismuth Nanocrystals. Chemistry of Materials 2011, 23, $137-144$.

(10) Yarema, M.; Kovalenko, M. V.; Hesser, G.; Talapin, D. V.; Heiss, W. Highly Monodisperse Bismuth Nanoparticles and Their Three-Dimensional Superlattices. Journal of the American Chemical Society 2010, 132, 1515815159.

(11) Briand, G. G.; Burford, N. Coordination complexes of bismuth(III) involving organic ligands with pnictogen or chalcogen donors. In Advances in Inorganic Chemistry; Academic Press, 2000; Vol. Volume 50, pp. 285-357. (12) Hiramatsu, H.; Osterloh, F. E. A Simple Large-Scale Synthesis of Nearly Monodisperse Gold and Silver Nanoparticles with Adjustable Sizes and with Exchangeable Surfactants. Chemistry of Materials 2004, 16, 2509- 2511. (13) Lindsjö, M.; Fischer, A.; Kloo, L. Anionic Diversity in lodobismuthate Chemistry. Zeitschrift für anorganische und allgemeine Chemie 2005, 631, 14971501. 
(14) Advances in Inorganic Chemistry; Academic Press, 1994.

(15) Hofmann, P. The surfaces of bismuth: Structural and electronic properties. Progress in Surface Science 2006, 81, 191-245.

(16) Xu, Z.; Shen, C.; Hou, Y.; Gao, H.; Sun, S. Oleylamine as Both Reducing Agent and Stabilizer in a Facile Synthesis of Magnetite Nanoparticles. Chemistry of Materials 2009, 21, 1778-1780.

(17) Shevchenko, E. V.; Bodnarchuk, M. I.; Kovalenko, M. V.; Talapin, D. V.; Smith, R. K.; Aloni, S.; Heiss, W.; Alivisatos, A. P. Gold/Iron Oxide Core/HollowShell Nanoparticles. Advanced Materials 2008, 20, 4323- 4329.

(18) Mazumder, V.; Sun, S. Oleylamine-Mediated Synthesis of Pd Nanoparticles for Catalytic Formic Acid Oxidation. Journal of the American Chemical Society 2009, 131, 4588-4589.

(19) Chen, M.; Feng, Y.-G.; Wang, X.; Li, T.-C.; Zhang, J.-Y.; Qian, D.-J. Silver Nanoparticles Capped by Oleylamine: Formation, Growth, and SelfOrganization. Langmuir 2007, 23, 5296-5304.

(20) Olson, E. A.; Efremov, M. Y.; Zhang, M.; Zhang, Z.; Allen, L. H. Sizedependent melting of Bi nanoparticles. Journal of Applied Physics 2005, 97, 034304-034304-9.

(21) Kharissova, O. V.; Kharisov, B. I. Nanostructurized Forms of Bismuth. Synthesis and Reactivity in Inorganic, Metal-Organic, and Nano-Metal Chemistry 2008, 38, 491-502. 
(22) Wang, Y.; Xia, Y. Bottom-Up and Top-Down Approaches to the Synthesis of Monodispersed Spherical Colloids of Low Melting-Point Metals. Nano Letters 2004, 4, 2047-2050.

\section{References from Chapter 5:}

(1) Lincourt, A. E.; Harrell, A.; Cristiano, J.; Sechrist, C.; Kercher, K.; Heniford, B. T. Retained Foreign Bodies After Surgery. Journal of Surgical Research 2007, $138,170-174$.

(2) Kanne, J. P.; Phillips, G. S. Anterior mediastinal gossypiboma. Radiology Case Reports 2011, 6.

(3) When Surgeons Leave Objects Behind.

http://well.blogs.nytimes.com/2012/09/24/when-surgeons-leave-objects- behind/ (accessed September 25, 2012).

(4) Misc Small Items. http://nothingleftbehind.org/Misc_items.html (accessed January 3,2013$)$.

(5) Palti-Wasserman, D.; Brukstein, A. M.; Beyar, R. P. Identifying and tracking a guide wire in the coronary arteries during angioplasty from X-ray images. IEEE Transactions on Biomedical Engineering 1997, 44, $152-164$.

(6) Materna, T.; Jolie, J.; Mondelaers, W.; Masschaele, B. Near K-edge measurement of the X-ray attenuation coefficient of heavy elements using a tuneable X-ray source based on an electron LINAC. Radiation Physics and 
Chemistry 2000, 59, 449-457.

(7) Allisy-Roberts, P. J.; Williams, J. R. Farr's Physics for Medical Imaging;

Elsevier Health Sciences, 2007. 


\section{Appendix A - Synthesis of Ethylene Glycol and Glucose Bismuth Nanoparticles and Applications as X-ray Opaque Inks}

Synthesis of bismuth nanoparticles in ethylene glycol with glucose as a surfactant yielded nanocrystals of distinct morphology and were used as a flowable X-ray attenuating inks. Bismuth nitrate $(500 \mu \mathrm{L}$ of $1 \mathrm{M})$ dissolved in ethylene glycol $(0.5 \mathrm{mM})$ was mixed with with $3.3 \mathrm{~g}$ glucose in $4.5 \mathrm{~mL}$ ethylene glycol and brought to specified synthetic temperature $\left(75-115{ }^{\circ} \mathrm{C}\right)$. Borane trimethylamine (BTMA) solution $(1.5 \mathrm{mM}, 1.5 \mathrm{~mL}$ of a $1 \mathrm{M}$ solution) melted in ethylene glycol (ca. $50{ }^{\circ} \mathrm{C}$ ) was added to induce nanocrystal growth. Particles were grown for 20 second and then poured onto iced water, collected by centrifugation and washed in ethanol and water. For synthesis in water, reduction took place at $50{ }^{\circ} \mathrm{C}$. Particles were suspended in water and dropcast on holey carbon copper support TEM grids for analysis. For XRD analysis particles were dried in films on glass microscope slides. A large particle preparation consisting of $15.4 \mathrm{~g} \mathrm{Bi}(\mathrm{NO} 3) 3-5 \mathrm{H} 2 \mathrm{O}$ and $31.5 \mathrm{~g}$ glucose mixed in $350 \mathrm{~mL}$ ethylene glycol and heated to $90-95{ }^{\circ} \mathrm{C}$ were reacted with $7.1 \mathrm{~g} \mathrm{BTMA}$ dissolved in $100 \mathrm{~mL}$ ethylene glycol for 20 seconds before quenching on ice. Particles were cleaned as previously described and were prepared for use as X-ray opaque ink. Particles were suspended in water solution and using a micropipette drawn onto and air

dried on a sheet of paper. X-ray radiographic image was collected at Portland Community College by Barb Smith at $90 \mathrm{keV}$. 


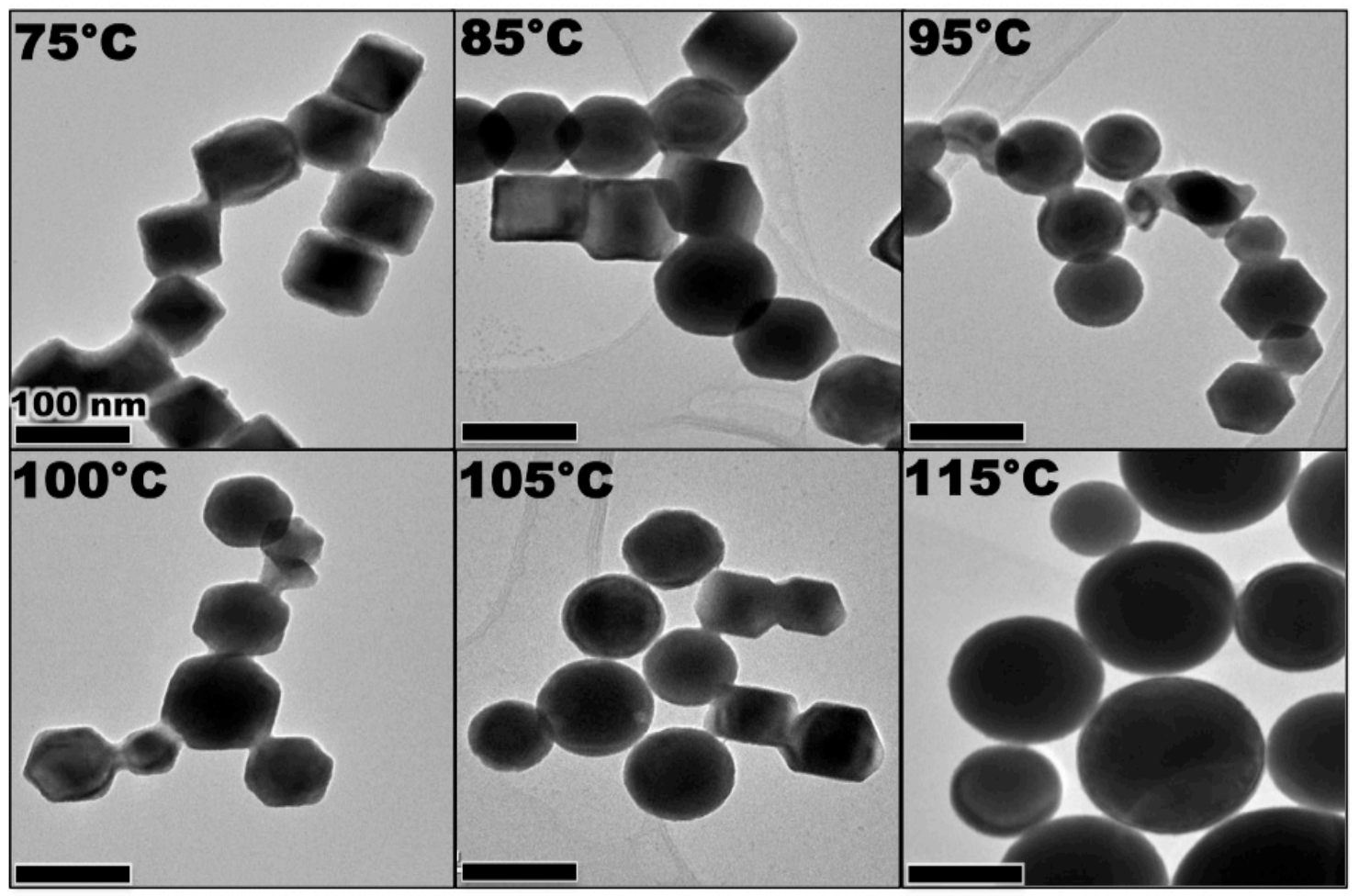

Figure A.1. Bismuth nanoparticles synthesized in ethylene glycol and surface stabilized with glucose. Particles were synthesized at temperatures noted in upper left corner. TEM images are representative of morphologies generally observed from each synthesis. 


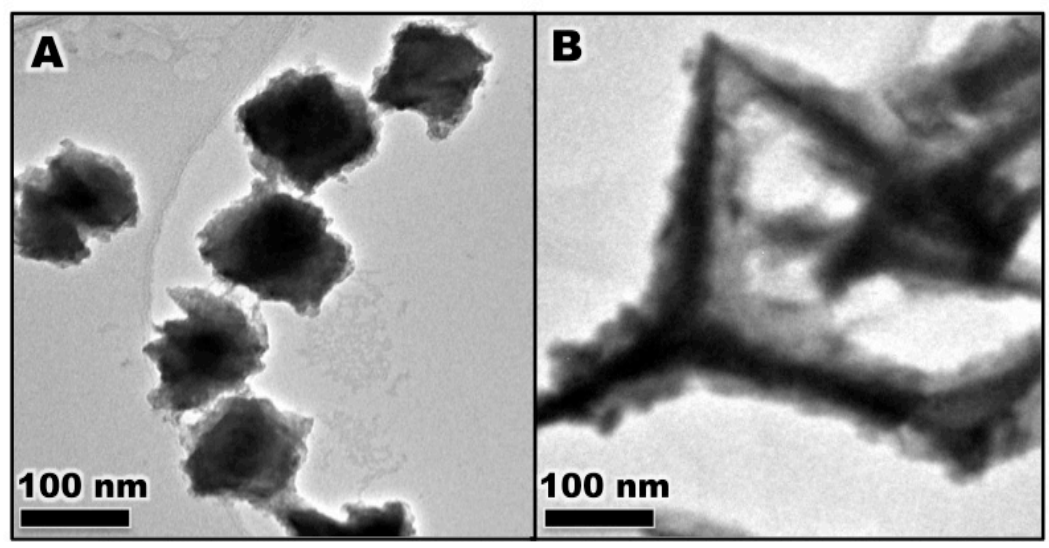

Figure A.2. Bismuth nanoparticles synthesized in the absence of glucose (A) or in water (B). Particles synthesized without glucose were not oxidatively stable in water and readily decomposed to a white bismuth oxide precipitate.

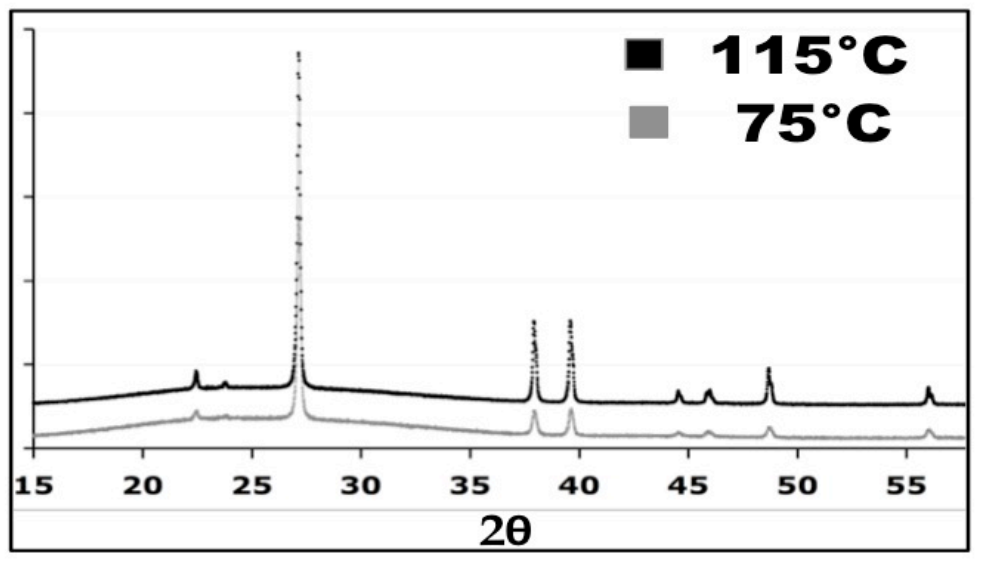

Figure A.3. XRD absorbance profile of bismuth nanoparticles with rounded morphologies $\left(115^{\circ} \mathrm{C}\right)$ or with pseudocubic morphologies $\left(75^{\circ} \mathrm{C}\right)$. Profiles display identical relative peak height reflection, suggesting an absence of preferential crystal orientation of dried samples. 


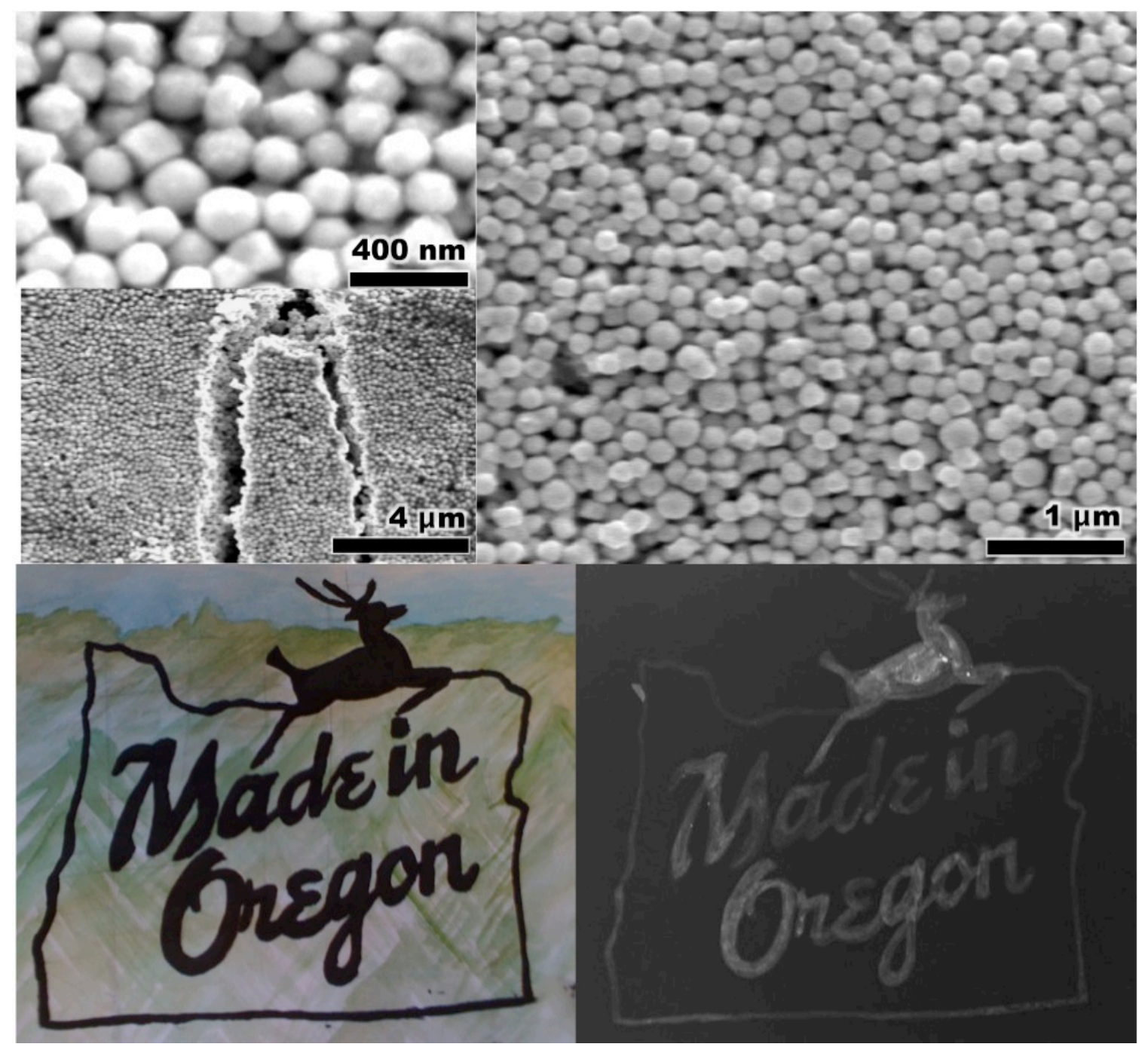

Figure A.4. Aqueous glucose-capped bismuth nanoparticle inks. Bismuth particles were synthesized and used as X-ray opaque ink for illustratrative application. SEM images of particles (top row) show no particle self-organization, but a high degree of self-adhesion indicated by formation of cracks. Photograph (lower left) and X-ray radiograph (lower right) of nanoparticle inks drawn on paper illustrate a potential application as flowable, heavy element nanoparticle X-ray opaque inks. 


\section{Appendix B. - Bismuth Nanoparticles Stabilized by a Variety of Reducing Sugars, and the pH Dependent Stability of Glucose Capped Particles}

Bismuth nanoparticles were synthesized and surface stabilized using mono- and di- saccharides to assess effects of surface stabilizers on nanocrystal morphology. A method, described in detail in Chapter 3 of this document, was modified for use of sugars other than glucose. In short, $10 \mathrm{mM} \mathrm{Bi(III)} \mathrm{nitrate} \mathrm{(100}$ $\mu \mathrm{mol})$ and $1 \mathrm{M}(0.9-3.42 \mathrm{~g})$ of the specified sugar were heated to $80^{\circ} \mathrm{C}$ in $10 \mathrm{~mL}$ 1,2-propane diol. Borane morpholine $(310 \mu \mathrm{Mol})$ suspended in $310 \mu \mathrm{L} \mathrm{1,2-}$ propanediol was added and the solution was allowed to react for $20 \mathrm{sec}$ to 5 minutes before dilution in ice water. The dihydroxyacetone solution reacted for 20 seconds, the fructose, ribose, and glucose solutions reacted for 60 seconds, and the maltose solution reacted for 5 minutes. In the presence of non-reducing sugars, namely xylitol, sorbitol, and trehalose, no blackening of reaction solution, indicating stabilization of bismuth particles, was observed. Particles were collected by centrifugation ( $4.4 \mathrm{rcf}, 20 \mathrm{~min}$ ) and washed in nanopure water prior to analysis. Transmission Electron Microscopy (TEM) was performed on an FEI Tecnai F-20 TEM operating at $200 \mathrm{kV}$. All particles were carried in water and dropcast onto holey carbon copper supported grids and dried for at least 2 hours at $150^{\circ} \mathrm{C}$ prior to imaging. 

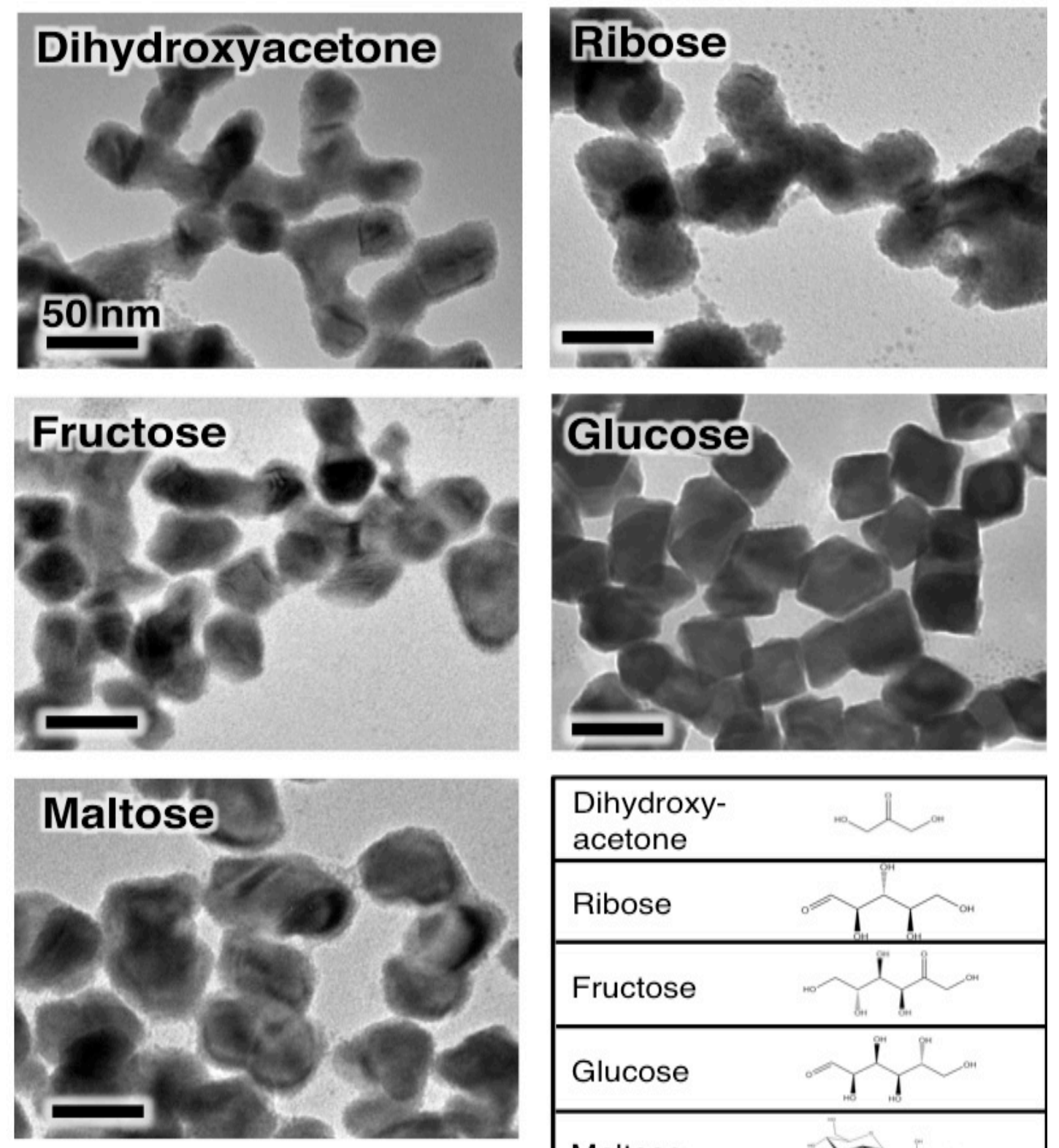

\begin{tabular}{|l|}
\hline $\begin{array}{l}\text { Dihydroxy- } \\
\text { acetone }\end{array}$ \\
\hline Ribose \\
\hline Fructose \\
\hline Glucose \\
\hline Maltose \\
\hline
\end{tabular}

Figure A.5. Bismuth nanoparticles synthesized in 1,2-propanediol with various reducing sugar surfactants. Chemical structures of surfactants are shown in lower right corner. All scale bars represent $50 \mathrm{~nm}$. 


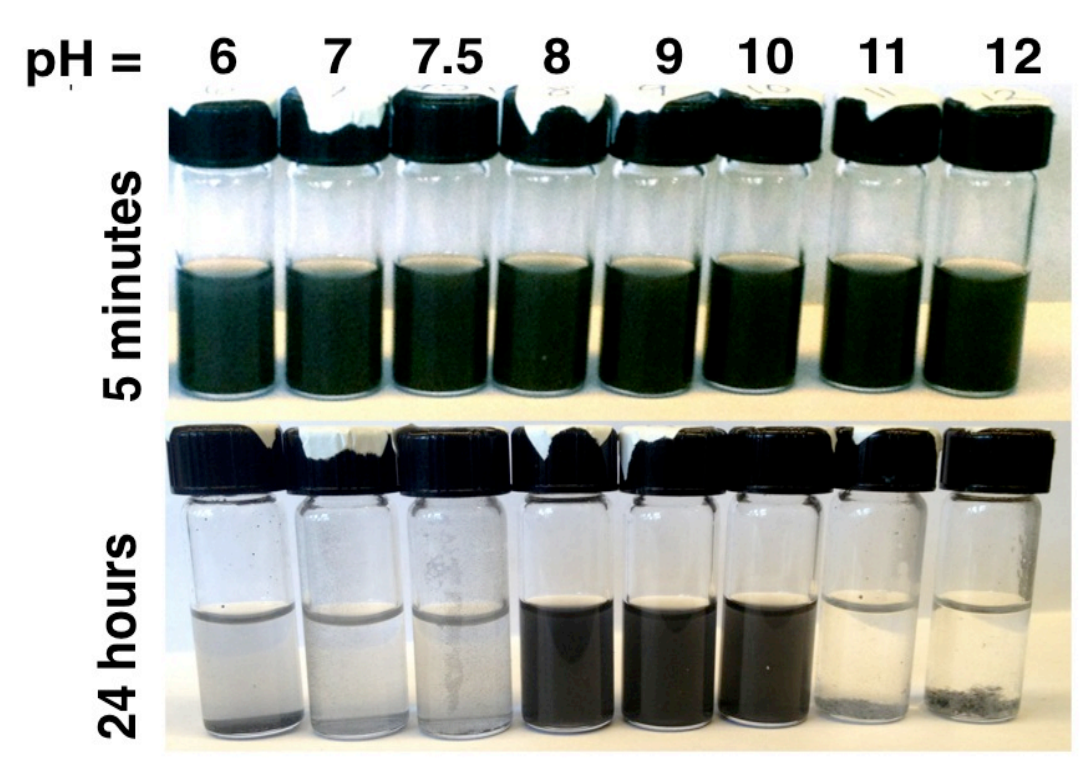

Figure A.6. pH dependent stability of glucose capped bismuth nanoparticles. Particles were mixed into phosphate solutions $(1 \mathrm{M})$ with $\mathrm{pH}$ values between 6 and 12, and photographed at 5 minutes and at 24 hours. Solutions with $\mathrm{pH}$ values between 8 and 10 appeared coloidally stable for greater than 1 month.

Glucose capped particles were synthesized as previously described (section 3.3). An unknown bismuth concentration of particles was assessed for pH stability in 1 molar phosphate buffered solutions ranging in $\mathrm{pH}$ from 6 to 12 . (Figure A.6) Briefly, $100 \mu \mathrm{L}$ of the nanoparticle solution, in water, was added to $1 \mathrm{~mL}$ of the phosphate solutions. Photographs were taken at 5 minutes, to illustrate dispersion, and vials were stored in the dark at room temperature over night. Another photograph was taken at 24 hours to illustrate oxidative decomposition of the particles in solutions with $\mathrm{pH}$ value of $6,7,7.5,11$, and 12 . Particles in solutions with a $\mathrm{pH}$ value of 8,9 , and 10 showed no indication of oxidative decomposition over night, and remained colloidally stable in solution for 
at least 1 month. As discussed in detail in Chapter 3 of this document, these $\mathrm{pH}$ dependent stability observations suggests glucose capped particles will be oxidatively decomposed when used as an in vivo X-ray contrast material. 